\title{
WestVirginiaUniversity
}

THE RESEARCH REPOSITORY @ WVU

Graduate Theses, Dissertations, and Problem Reports

2015

\section{Synthesis, characterization and photocatalytic activity of Au-ZnO heterostructures}

Oshadha Kanchana Ranasingha

Follow this and additional works at: https://researchrepository.wvu.edu/etd

\section{Recommended Citation}

Ranasingha, Oshadha Kanchana, "Synthesis, characterization and photocatalytic activity of Au-ZnO heterostructures" (2015). Graduate Theses, Dissertations, and Problem Reports. 6473.

https://researchrepository.wvu.edu/etd/6473

This Dissertation is protected by copyright and/or related rights. It has been brought to you by the The Research Repository @ WVU with permission from the rights-holder(s). You are free to use this Dissertation in any way that is permitted by the copyright and related rights legislation that applies to your use. For other uses you must obtain permission from the rights-holder(s) directly, unless additional rights are indicated by a Creative Commons license in the record and/ or on the work itself. This Dissertation has been accepted for inclusion in WVU Graduate Theses, Dissertations, and Problem Reports collection by an authorized administrator of The Research Repository @ WVU.

For more information, please contact researchrepository@mail.wvu.edu. 


\title{
Synthesis, characterization and photocatalytic activity of
}

\section{Au-ZnO heterostructures}

\author{
Oshadha Kanchana Ranasingha
}

Dissertation submitted to the
Eberly College of Arts and Sciences at West Virginia University in partial fulfillment of the requirements for the degree of

\section{Doctor of Philosophy \\ in \\ Physics}
James P. Lewis, Ph. D., Chair Alan D. Bristow, Ph. D.
Tudor Stanescu, Ph. D. Cheng Cen, Ph. D.
Christopher Matranga, Ph. D.

\author{
Department of Physics and Astronomy \\ Morgantown, West Virginia \\ 2015
}

Keywords: Au-ZnO heterostructures, plasmonic heating, photocatalysts, Au nanoparticles, $\mathrm{CO}_{2}$ conversion

Copyright 2015 Oshadha Ranasingha 


\section{Abstract \\ Synthesis, characterization, and photocatalytic activity of Au-ZnO heterostructures Oshadha Kanchana Ranasingha}

Heterostructures of semiconductor metal oxides and metals have been studied extensively due to the synergistic photocatalytic activity they display. Gold-Zinc oxide ( $\mathrm{Au}-\mathrm{ZnO})$ heterostructured photocatalysts are one of the most widely studied photocatalysts due to the high photocatalytic activity of $\mathrm{ZnO}$ and the ability of $\mathrm{Au}$ to transfer photoexcited electrons away from $\mathrm{ZnO}$, which leads to enhanced carrier separation and improved catalytic activity. Au heterostructuring is also known to alter the electronic structure of $\mathrm{ZnO}$ leading to better photochemical band alignment and a decreased work function which both contribute to enhanced catalytic activity. The use of Au to form heterostructures with $\mathrm{ZnO}$ also opens the possibility of using its plasmonic activity for engineering the photophysics of this system through plasmonic heating or optical field enhancement. As such, it is interesting to synthesize a variety $\mathrm{Au}-\mathrm{ZnO}$ heterostructures and correlate the catalytic activity of these materials with structural details such as nanoparticle morphology, lattice dynamics at the interface, and the general crystallinity of the two materials forming the heterostructure.

In this work, I have synthesized, characterized, and investigated two types of $\mathrm{Au}-\mathrm{ZnO}$ heterostructured catalysts and evaluated their activity for carbon dioxide $\left(\mathrm{CO}_{2}\right)$ conversion and waste-water remediation applications. The $\mathrm{Au}-\mathrm{ZnO}$ catalysts were characterized using Transmission Electron Microscopy, Scanning Electron Microscopy, X-Ray Diffractometry, Raman spectroscopy, UV-Vis absorption spectroscopy and Fourier Transform Infrared spectroscopy. Initial efforts focused on simple heterostructures made by soaking nanocrystalline 
$\mathrm{ZnO}$ in a $\mathrm{Au}$ salt with a subsequent heat treatment to reduce the Au to its metallic state. This resulted in $\sim 20 \mathrm{~nm}$ Au particles dispersed on $\mathrm{ZnO}$ at $\sim 5 \mathrm{wt} \%$ loading. Plasmonic excitation of the $\mathrm{Au}$ nanoparticles also heats the $\mathrm{ZnO}$ substrate it is grown on up to $\sim 600{ }^{0} \mathrm{C}$ and drives the catalytic conversion of $\mathrm{CO}_{2}$ and hydrogen $\left(\mathrm{H}_{2}\right)$ to methane $\left(\mathrm{CH}_{4}\right)$, carbon monoxide $(\mathrm{CO})$, and water $\left(\mathrm{H}_{2} \mathrm{O}\right)$ on the $\mathrm{ZnO}$ portion of the heterostructure. In addition, I have also investigated methods to increase the $\mathrm{Au}-\mathrm{ZnO}$ ratio and to improve the degree of contact between these two materials. For these efforts, $\mathrm{ZnO}$ nanopyramids were grown on $\mathrm{Au}$ seeds using a wet-chemical method. Electron microscopy was used to characterize an unusual and large lattice expansion for $\mathrm{ZnO}$ at the $\mathrm{Au}$ interface in these samples. Optical measurements of these samples point to subtle electronic structure changes caused by the Au that improve band alignment for the production of radicals from photoexcited carriers in the $\mathrm{ZnO}$. The improved production of radicals in turn leads to an enhanced photodegradation of organic contaminants in water. In addition to experimental work, computational calculations were performed in order to calculate vibrational modes, phonon spectral density and plasmon relaxation time constants. Two $\mathrm{Au}$ clusters $\left(\mathrm{Au}_{20}\right.$ and $\left.\mathrm{Au}_{55}\right)$ which contain 20 and 55 of $\mathrm{Au}$ atoms respectively were used for the calculations. 
Dedicated to my father Mr. Danasiri Ranasinghe and my mother Mrs. Chandralatha Ranasinghe. 


\section{Acknowledgment}

First of all, I would like to thank my advisor Dr. James P. Lewis for his guidance, support and encouragement that made me who I am today. He always guided me through the correct path in my education and research career. It was a great pleasure to work with Dr. Lewis, and his knowledge and dedication was an inspiration for me to complete my work on time.

I would also like to thank Dr. Christopher Matranga at NETL-Pittsburgh for his guidance and tremendous support on my experimental work carried out at the NETL-Pittsburgh. Also, I would like to thank Dr. Congjun Wang and all the members of Matranga group at NETL-Pittsburgh for their invaluable support extended to me throughout the years. It was a great experience to work

with the experts like them who were willing to help and teach me all the experimental techniques.

I would also like to extend my gratitude to Dr. Alan Bristow, Dr. Tudor Stanescu and Dr. Cheng Cen for serving in my PhD committee and for their guidance and kind support.

I would like to thank Dr. Hong Wang, Dr. Barry Haycock and all the members of Lewis group for their invaluable support for my computational work.

I acknowledge the WVU shared facilities and its great staff for their tremendous support given to me to make this work a success. Furthermore, I extend my appreciation to the administrative staff of the WVU Physics department for all their help and support. 
In addition, I would like to extend my gratitude to all my friends at the WVU Physics and Astronomy department for the nice times we had together. Also, I am so thankful for the support of my Sri Lankan friends in Morgantown, who were more like family to me.

I would also like to thank Dr. Xingbo Liu and his wonderful research group for all the help that was given to complete some experiments at MAE, WVU.

At this moment I would like to thank all my teachers and friends who help to achieve success since grade one to end of the graduate school.

Finally, I would like to express my sincere gratitude to my wonderful parents and my brother for their love and dedication. I would never be able to come this far without their tremendous support. Lastly, I would like to thank my loving wife Kaushalya de Silva for always being there for me through the best and the worst. 


\section{Table of Contents}

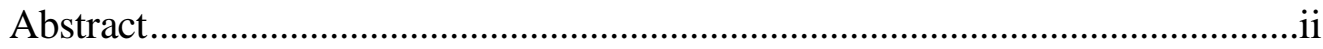

Acknowledgments .............................................................................................iv

Table of Contents ...........................................................................................

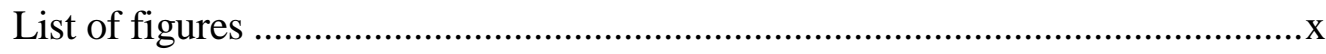

List of tables .......................................................................................... Xvii

Chapter 01: Introduction...................................................................................................1

1.1 Heterogeneous photocatalysts.............................................................

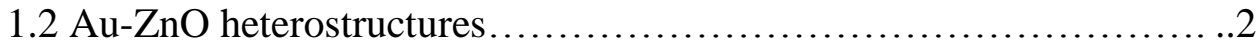

1.2.1 Surface plasmons..........................................................................

1.2.2 Surface plasmons induced hot electron assisted photocatalytic activity of $\mathrm{Au}-\mathrm{ZnO}$......................................................................

1.2.3 Photocatalytic activity of Au-ZnO under UV illumination ...........8

1.3 Plasmonic heat generation..................................................................... 10

1.3.1 Importance of $\mathrm{ZnO}$ for plasmonic heating assisted

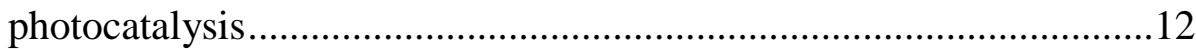

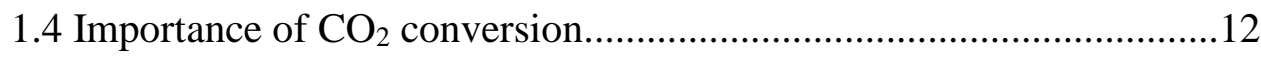

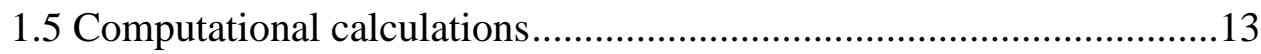

1.6 Structure of the dissertation ............................................................ 14

Chapter 02:Experimental Techniques......................................................................22

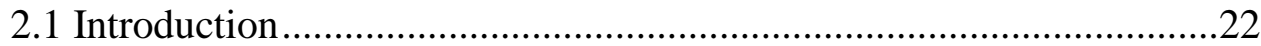

2.2 Transmission Electron Microscopy (TEM) ………………………......22

2.3 Scanning Electron Microscopy (SEM) ………………………….... 25

2.4 Diffuse reflectance spectroscopy ....................................................... 27

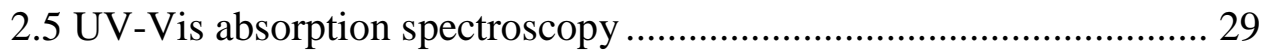

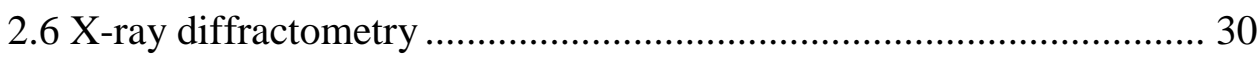

2.7 Fourier Transform Infra-Red spectroscopy (FTIR) ...............................32

2.8 Gas Chromatography (GC) ..............................................................3

2.8.1 Thermal Conductivity Detector (TCD) ........................................33

2.8.2 Flame Ionization Detector (FID) .................................................34 


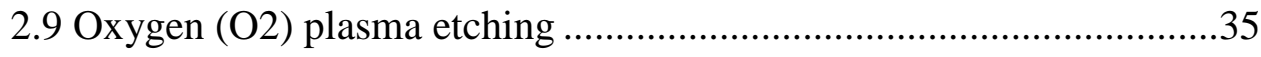

2.10 Raman spectroscopy ………………………………......................36

Chapter 03: Vibrational modes and phonon spectral density of Auzo

clusters.............................................................................................................41

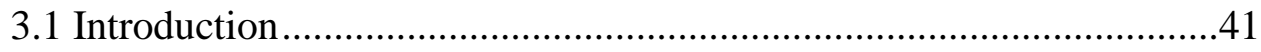

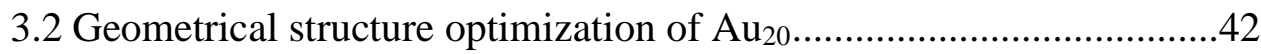

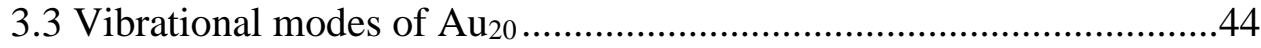

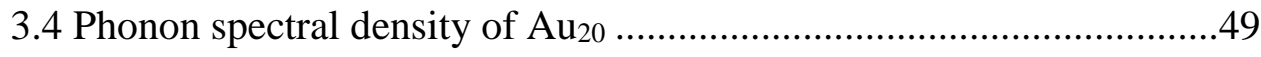

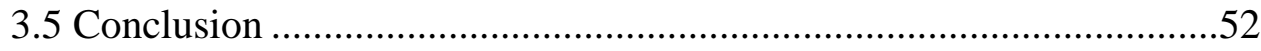

Chapter 04: Phonon induced relaxation of plasmon excitation in

Aus5 clusters................................................................................................55

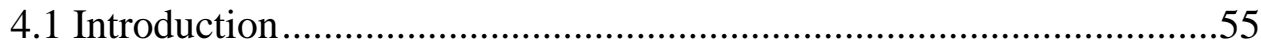

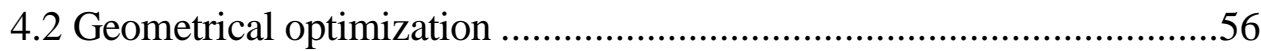

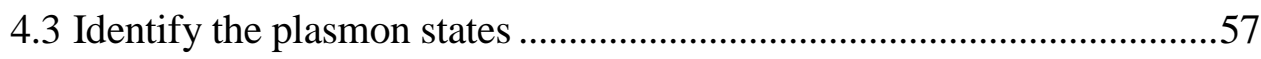

4.4 Non-adiabatic molecular dynamics (NAMD) simulations.......................60

4.4.1 Computational method .................................................................60

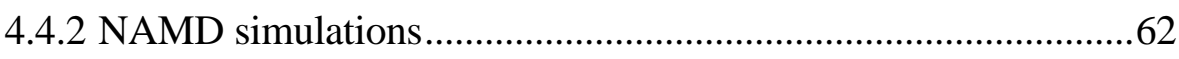

4.4.3 Conical intersections and electron hopping .................................67

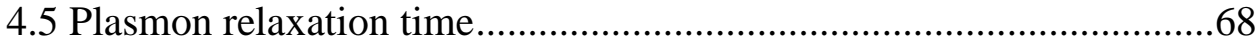

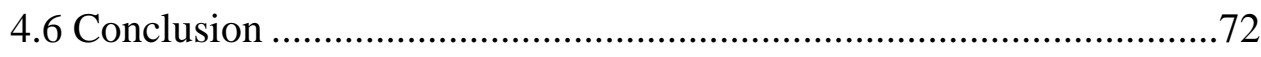

Chapter 05: Plasmonic heating assisted $\mathrm{CO}_{2}$ reduction using

Au-ZnO heterostructures ..........................................................................76

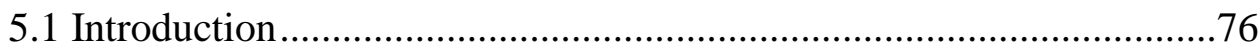

5.2 Light absorption and heat generation from the Au nanoparticles ...........77

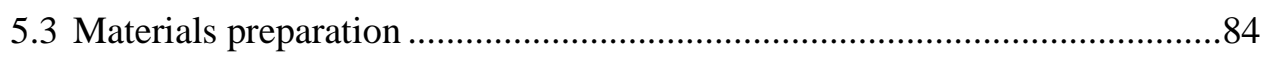

5.3.1 Synthesis of $\mathrm{Au}-\mathrm{ZnO}$ heterostructured catalysts ..........................85

5.4 Characterization of the $\mathrm{Au}-\mathrm{ZnO}$ heterostructures ......................................86

5.4.1 Diffuse reflectance spectroscopy characterization ........................86

5.4.2 Scanning Electron Microscopy characterization ..........................90 
5.4.3 BET surface area measurements ............................................92

5.4.4 Transmission Electron Microscopy characterization .................94

5.4.4 X-Ray Diffraction patterns characterization..............................95

5.5 Local temperature estimation studies of the $\mathrm{Au}-\mathrm{ZnO}$ heterostructures..96

5.5.1 Optical phonon modes of $\mathrm{ZnO}$................................................97

5.5.2 Temperature dependent intensity dependent

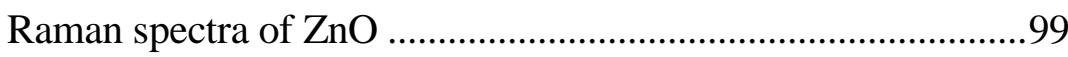

5.5.3 Theoretical model for the linewidth broadening of the

$E_{2}($ high) mode.................................................................. 102

5.5.4 Raman spectra of $\mathrm{ZnO}$ and $\mathrm{Au}-\mathrm{ZnO}$ at room temperature under different laser excitation intensities ..............................104

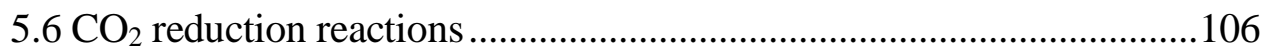

5.6.1 Temperature programmed reactions .......................................110

5.6.2 Plasmonic heating assisted catalytic activity of $\mathrm{Au}-\mathrm{ZnO}$........113

5.7 Conclusion

\section{Chapter 06: Synthesis, characterization, and photocatalytic activity of}

Au-ZnO nanopyramids...............................................................120

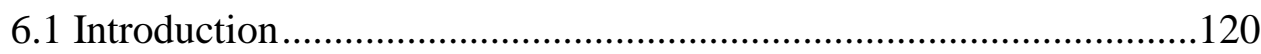

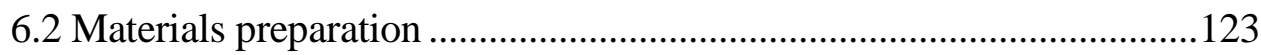

6.2.1 Synthesis of Au nanoparticles ................................................123

6.2.2 Synthesis of Au-ZnO nanopyramids .....................................124

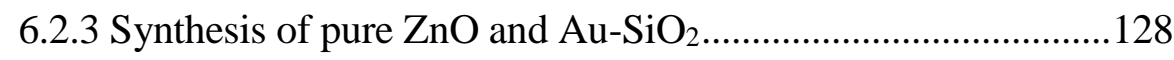

6.3 Methylene blue degradation reactions..............................................129

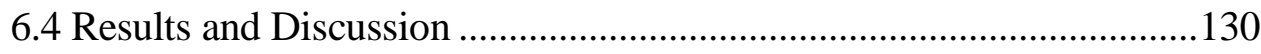

6.4.1 Characterization of nanoparticle samples using

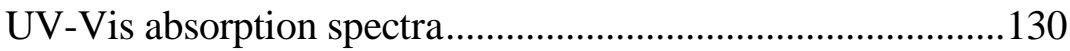

6.4.2 Characterization of TEM and HRTEM images........................133

6.4.3 Characterization of X-Ray Diffraction patterns and

Rietveld analysis............................................................. 141

6.4.4 Photocatalytic performance ..................................................144 
6.5 Conclusions 


\section{$\underline{\text { List of Figures }}$}

Figure 1.1 Comparison of solar intensity as function of wavelength 3

Figure 1.2 Comparison of diffuse reflectance spectra of pure $\mathrm{ZnO}$ and $\mathrm{Au}-\mathrm{ZnO} \quad 3$

Figure 1.3 (a) The field distributions of electron charge density fluctuations in 5 metal-dielectric interface (b) The exponential decay of the electric filed in the direction $\mathrm{Z}$ at the metal-dielectric interface with the characteristic lengths

Figure 1.4 Calculated extinction (black), absorption (red), and scattering (blue) spectra for Ag nanoparticles of different shapes: (a) sphere (b) cube (c) tetrahedron (d) octahedron and (e) triangular plate. (f) Calculated extinction spectra of rectangular Ag nano bars with different aspect ratios of 2 (black), 3 (red), and 4 (blue)

Figure 1.5 Proposed mechanism for the photodegradation of organic dyes with $\mathrm{Au}-\mathrm{ZnO}$ heterostructures under visible light illumination

Figure 1.6 Proposed mechanism for the photodegradation of organic dyes with $\mathrm{Au}-\mathrm{ZnO}$ heterostructures under UV light illumination

Figure 1.7 Calculated heat generation spectra for different sizes of $\mathrm{Au}$ nanoparticles. Aspect ratios starting from sphere 1:1, 1.4:1, 2:1, and $3: 1$

Figure 2.1 The schematic diagram of the Transmission Electron Microscope

Figure 2.2 The schematic diagram of the Scanning Electron Microscope 26

Figure 2.3 Illustration of the sample interaction volume and corresponding 26 particles emitted from sample depth

Figure 2.4 The schematic diagram showing the difference between specular reflection and diffuse reflection

Figure 2.5 The schematic diagram of the UV-Vis spectrophotometer 30

Figure 2.6 The schematic diagram of the X-ray generation from high energy 31

Figure 2.7 The schematic diagram of the generation of FTIR spectrum 32

Figure 2.8 The schematic diagram of the thermal conductivity detector 34

Figure 2.9 The schematic diagram of the flame ionization detector 34

Figure 2.10 The schematic diagram of the $\mathrm{O}_{2}$ plasma etching 35

Figure 2.11 The schematic diagram of the stoke and anti-stokes lines 36

Figure 3.1 Optimized tetrahedral structure of $\mathrm{Au}_{20}$, 'a','c' and 'e' represent apex, 43 center and edge atoms 
Figure 3.2 Variation of the total energy during the geometrical structure optimization

Figure 3.3 Vibrational modes of $\mathrm{Au}_{20}$ cluster 46

Figure 3.4 Schematic diagram of a highest vibrational mode $\left(4^{\text {th }}\right.$ mode of $\mathrm{A}_{1} \quad 47$ symmetry)

Figure 3.5 Schematic diagram of a Raman breathing mode ( $3^{\text {rd }}$ mode of $A_{1}$ symmetry)

Figure 3.6 Schematic diagram of a degenerate $6^{\text {th }}$ mode of $T_{2}$ symmetry (mode 49, 50 and 51)

Figure 3.7 VAC function for 4 different MD simulations $\quad 50$

Figure 3.8 Average VAC function which used to calculate the phonon spectral 50 density

Figure 3.9 Phonon spectral density spectrum of $\mathrm{Au}_{20}$ cluster. Red lines show the 51 distinct peaks

Figure 4.1 Icosahedral structure of $\mathrm{Au}_{55}$

Figure 4.2 The variation of total energy of the $\mathrm{Au}_{55}$ cluster during the geometrical 57 structure optimization

$\begin{array}{lll}\text { Figure 4.3 } & \text { HOMO state }\left(\mathrm{S}_{303}\right) & 58\end{array}$

$\begin{array}{lll}\text { Figure 4.4 LUMO state }\left(\mathrm{S}_{304}\right) & 58\end{array}$

Figure $4.5 \quad$ State $305\left(\mathrm{~S}_{305}\right) \quad 58$

$\begin{array}{lll}\text { Figure } 4.6 & \text { Plasmon state }\left(\mathrm{S}_{338}\right) & 58\end{array}$

$\begin{array}{lll}\text { Figure } 4.7 & \text { Plasmon state }\left(\mathrm{S}_{339}\right) & 58\end{array}$

$\begin{array}{lll}\text { Figure } 4.8 & \text { Plasmon state }\left(\mathrm{S}_{341}\right) & 58\end{array}$

Figure $4.9 \quad$ Plasmon state $\left(\mathrm{S}_{342}\right) \quad 59$

Figure 4.10 Plasmon state $\left(\mathrm{S}_{343}\right) \quad 59$

Figure 4.11 Density of states of $\mathrm{Au}_{55}$ and energy of plasmon states 60

Figure 4.12 The populations of all the states between HOMO state and initial 63 plasmon state $\mathrm{S}_{338}$

Figure 4.13 The populations of all the states between HOMO state and initial 63 plasmon state $\mathrm{S}_{339}$

Figure 4.14 The populations of all the states between HOMO state and initial 63 plasmon state $\mathrm{S}_{341}$ 
Figure 4.15 The populations of all the states between HOMO state and initial plasmon state $\mathrm{S}_{342}$

Figure 4.16 The populations of all the states between HOMO state and initial plasmon state $\mathrm{S}_{343}$

Figure 4.17 Average maximum populations at each state for five different initial plasmon states

Figure 4.18 The populations of state $S_{343}$ for initial plasmon state $S_{338}$

Figure 4.19 The populations of state $S_{343}$ for initial plasmon state $S_{339}$

Figure 4.20 The populations of state $S_{343}$ for initial plasmon state $S_{341}$

Figure 4.21 The populations of state $S_{343}$ for initial plasmon state $S_{339}$

Figure 4.22 The populations of state $S_{343}$ for initial plasmon state $S_{343}$

Figure 4.23 The evolution of energies of state $S_{332}, S_{331}$ and $S_{330}$ along one 68 trajectory of initial plasmon state $S_{338}$

Figure 4.24 The Gaussian fit for the $\left[1-S_{\text {HOMO }}\right]$ data 69

Figure 4.25 Relaxation time constants for initial plasmon state $S_{338}$

Figure 4.26 Relaxation time constants for initial plasmon state $S_{339} \quad 70$

Figure 4.27 Relaxation time constants for initial plasmon state $S_{341}$

Figure 4.28 Relaxation time constants for initial plasmon state $S_{342} \quad 70$

Figure 4.29 Relaxation time constants for initial plasmon state $S_{343}$

Figure 5.1 Real part of the wavelength dependent dielectric constant of $\mathrm{Au} \quad 80$

Figure 5.2 Imaginary part of the wavelength dependent dielectric constant of $\mathrm{Au} \quad 80$

Figure 5.3 Absorption cross section of $20 \mathrm{~nm}$ spherical shaped Au nanoparticle in 82 air and in $\mathrm{ZnO}$ media

Figure 5.4 Temperature increase of $20 \mathrm{~nm}$ spherical shaped Au nanoparticle embedded in $\mathrm{ZnO}$

Figure 5.5 Temperature increase of different sizes of spherical shaped $\mathrm{Au}$ nanoparticles embedded in $\mathrm{ZnO}$

Figure 5.6 Diffuse Reflectance spectra for 5\% Au-ZnO heterostructure samples 87 at different annealing temperatures

Figure 5.7 Diffuse Reflectance spectra for $\mathrm{Au}-\mathrm{ZnO}$ heterostructure samples with different $\mathrm{Au}$ concentration annealed at $200{ }^{\circ} \mathrm{C}$

Figure 5.8 Comparison of Diffuse Reflectance spectra for pure $\mathrm{ZnO}$ and $5 \% \mathrm{Au}-$ $\mathrm{ZnO}$ 
Figure 5.9 Comparison of Diffuse Reflectance spectra for 3 different batches of 90 $5 \% \mathrm{Au}-\mathrm{ZnO}$

Figure 5.10 Comparison of SEM images at the same position of Au-ZnO sample 91 using two different detectors

Figure $5.11 \quad 5 \% \mathrm{Au}-\mathrm{ZnO}$ annealed at $100{ }^{\circ} \mathrm{C} \quad 92$

Figure $5.12 \quad 5 \% \mathrm{Au}-\mathrm{ZnO}$ annealed at $200{ }^{\circ} \mathrm{C} \quad 92$

Figure $5.13 \quad 5 \% \mathrm{Au}-\mathrm{ZnO}$ annealed at $300{ }^{\circ} \mathrm{C} \quad 92$

Figure 5.14 5\% Au- $\mathrm{ZnO}$ annealed at $400{ }^{\circ} \mathrm{C} \quad 92$

Figure $5.15 \quad 5 \% \mathrm{Au}-\mathrm{ZnO}$ annealed at $600{ }^{\circ} \mathrm{C} \quad 93$

Figure $5.16 \quad 5 \% \mathrm{Au}-\mathrm{ZnO}$ annealed at $800{ }^{\circ} \mathrm{C} \quad 93$

Figure 5.17 Different positions of 5\% Au-ZnO sample 93

Figure 5.18 TEM image of 5\% Au-ZnO sample 94

Figure 5.19 HRTEM image of 5\% Au-ZnO sample 95

Figure 5.20 X-Ray Diffraction patterns of 5\% Au-ZnO 96

Figure 5.21 Crystal structure of wurtzite $\mathrm{ZnO}$. Grey color represents $\mathrm{Zn}$ atoms and 97 red color represents $\mathrm{O}$ atoms. Highlighted atoms are inside the unit cell

Figure 5.22 Eigen vectors of the $\mathrm{ZnO}$ optical phonon modes. The bold arrows 98 represent the dominating displacement vectors, while grey circles represent $\mathrm{Zn}$ atoms and white circles represent $\mathrm{O}$ atoms. Superscripts 1 and 2 showed the low and high frequencies.

Figure 5.23 Raman spectra of $\mathrm{ZnO}$ at different temperatures 100

Figure 5.24 Raman spectra of $\mathrm{ZnO}$ at different laser intensities 100

Figure 5.25 Raman shift and linewidth variation of $E_{2}($ high) mode of $\mathrm{ZnO}$ at 101

Figure 5.26 Raman shift and linewidth variation of $E_{2}($ high) mode of $\mathrm{ZnO}$ at 102 laser intensities

Figure 5.27 Linewidth of $E_{2}($ high) mode of $\mathrm{ZnO}$ as a function of temperature 103 (black dots) under $2.6 * 10^{5} \mathrm{~W} / \mathrm{m}^{2} \mathrm{cw} 532 \mathrm{~nm}$ laser excitation. The red line is the fit based on the theoretical equation

Figure 5.28 Linewidth of $E_{2}($ high) mode of $\mathrm{ZnO}$ (black squares) and $\mathrm{Au}-\mathrm{ZnO}$ (red dots) as a function of different intensities of cw $532 \mathrm{~nm}$ laser excitation. The red line is a linear fit of the linewidth variation of the $\mathrm{Au}-\mathrm{ZnO}$ 
Figure 5.29 Estimated local temperature of $\mathrm{Au}-\mathrm{ZnO}$ under different laser intensities

Figure 5.30 The schematic diagram of laser illumination and homemade photocatalysis cell

Figure 5.31 $\mathrm{CO}_{2}$ conversion rate at different laser intensities with $\mathrm{Au}-\mathrm{ZnO}$, with

108 the corresponding local temperature due to the plasmonic heating as the top $x$-axis

Figure 5.32 $\mathrm{CO}$ production rate at different laser intensities with $\mathrm{Au}-\mathrm{ZnO}$, with the 108 corresponding local temperature due to the plasmonic heating as the top $x$-axis

Figure 5.33 $\mathrm{CH}_{4}$ production rate at different laser intensities with $\mathrm{Au}-\mathrm{ZnO}$, with 109 the corresponding local temperature due to the plasmonic heating as the top $x$-axis

Figure 5.34 CO production detected by a mass spectrometer as function of temperature for $\mathrm{ZnO}, \mathrm{Au}-\mathrm{ZnO}$ and $\mathrm{Au}-\mathrm{SiO}_{2}$

Figure $5.35 \mathrm{CH}_{4}$ production detected by a mass spectrometer as function of

112 temperature for $\mathrm{ZnO}, \mathrm{Au}-\mathrm{ZnO}$ and $\mathrm{Au}-\mathrm{SiO}_{2}$

Figure 5.36 The experimental $\mathrm{CO}$ (blue squares) and $\mathrm{CH} 4$ (red circles) selectivity as a function of laser intensity and corresponding catalyst temperature in top $x$-axis

Figure 5.37 The $\mathrm{CO}_{2}$ conversion rate for $\mathrm{Au}-\mathrm{ZnO}$ catalyst after 5 hour laser illumination cycle

Figure 6.1 Schematic diagram of $5 \% \mathrm{Au}-\mathrm{ZnO}$

Figure 6.2 Schematic diagram of $\mathrm{Au}-\mathrm{ZnO}$ nanopyramids

Figure 6.3 TEM image of $\mathrm{Au}-\mathrm{ZnO}$ (sample 21)

Figure 6.4 TEM image of $\mathrm{Au}-\mathrm{ZnO}$ (sample 22)

Figure 6.5 TEM image of $\mathrm{Au}-\mathrm{ZnO}$ (sample 23)

Figure 6.6 TEM image of $\mathrm{Au}-\mathrm{ZnO}$ (sample 26)

Figure 6.7 TEM image of $\mathrm{Au}-\mathrm{ZnO}$ (sample 27)

$\begin{array}{lll}\text { Figure 6.8 } & \text { TEM image of } \mathrm{Au}-\mathrm{ZnO} \text { (sample 29) } & 128\end{array}$

$\begin{array}{lll}\text { Figure 6.9 } & \text { TEM image of } \mathrm{Au}-\mathrm{ZnO} \text { (sample 30) } & 128\end{array}$

Figure 6.10 TEM image of Au-ZnO (sample 31) 128

Figure 6.11 FTIR spectra of pure $\mathrm{ZnO}$ before (black) and after (red) the plasma 129 etching 
Figure 6.12 FTIR spectra of $\mathrm{Au}-\mathrm{ZnO}$ before (black) and after (red) the plasma etching

Figure 6.13 UV-Vis absorption spectra for Au seeds (black), pure $\mathrm{ZnO}$ (blue) and $\mathrm{Au}-\mathrm{ZnO}$ heterostructures (red).

Figure 6.14 Comparison of Au nanoparticle suspended in hexane and full embedded in $\mathrm{ZnO}$

Figure 6.15 Tauc plots for the direct (black) and indirect (blue) band gap estimations of pure $\mathrm{ZnO}$ and $\mathrm{Au}-\mathrm{ZnO}$

Figure 6.16 TEM images of pure $\mathrm{ZnO}$ nanopyramids

Figure 6.17 TEM images of Au seeds

Figure 6.18 TEM images of $\mathrm{Au}-\mathrm{ZnO}$ nanopyramids 135

Figure 6.19 HRTEM image of the Au-ZnO interface 136

Figure 6.20 a) Schematic diagram for possible orientations of Au nanoparticles on 138 the basal surface of the $\mathrm{ZnO}$ nanopyramids (b) TEM images of different basal surfaces of the $\mathrm{ZnO}$ nanopyramids.

Figure 6.21 d-spacing analyzing method of $\mathrm{ZnO}(002)$ lattice plane 138

$\begin{array}{lll}\text { Figure } 6.22 & \mathrm{Au}-\mathrm{ZnO} \text { interface } 1 & 139\end{array}$

Figure 6.23 $\mathrm{Au}-\mathrm{ZnO}$ interface 2 139

$\begin{array}{lll}\text { Figure 6.24 } & \mathrm{Au}-\mathrm{ZnO} \text { interface } 3 & 139\end{array}$

$\begin{array}{lll}\text { Figure 6.25 } & \mathrm{Au}-\mathrm{ZnO} \text { interface } 4 & 139\end{array}$

Figure 6.26 Line scan along the $\mathrm{Au}-\mathrm{ZnO}$ interface with average lattice spacings 140 for the $\mathrm{ZnO}$ (002) plane in the regions shown.

Figure 6.27 Average $d$-spacing of $\mathrm{ZnO}(002)$ at different distances from the interface. Error bars were determined by analyzing 9 different structures.

Figure 6.28 Comparison of X-ray diffraction patterns of pure $\mathrm{ZnO}$ and $\mathrm{Au}-\mathrm{ZnO}$ nanopyramids

Figure 6.29 High resolution XRD patterns with calculated Rietveld pattern for 143 pure $\mathrm{ZnO}$

Figure 6.30 High resolution XRD patterns with calculated Rietveld pattern for Au- 144 $\mathrm{ZnO}$

Figure 6.31 UV-Vis absorption spectra for MB degradation using $\mathrm{Au}-\mathrm{ZnO}$ with $\lambda \quad 145$ $>295 \mathrm{~nm}$ 
Figure 6.32 $\mathrm{MB}$ degradation results for $\mathrm{Au}-\mathrm{ZnO}$ (black), $\mathrm{ZnO}$ (red), $\mathrm{MB}$ solution

(no catalyst) (blue) and $\mathrm{Au}-\mathrm{SiO}_{2}$ (magenta) with $\lambda>295 \mathrm{~nm}$

illumination

Figure 6.33 Linear fit for $\ln \left(C / C_{0}\right)$ of $\mathrm{MB}$ versus time for $\mathrm{Au}-\mathrm{ZnO}$ (a) under $\lambda>\quad 146$ $295 \mathrm{~nm}$ illumination (b) under $\lambda>400 \mathrm{~nm}$ illumination

Figure 6.34 Wavelength dependent MB degradation with $\mathrm{Au}-\mathrm{ZnO} \quad 148$

$\begin{array}{lll}\text { Figure 6.35 first order rate constants for MB degradation with } \mathrm{Au}-\mathrm{ZnO} \text { and } \mathrm{TiO}_{2} & 148\end{array}$ using different longpass filters

Figure 6.36 Approximate band alignment of $\mathrm{Au}-\mathrm{ZnO}$. Potentials are with respect 150 to NHE 


\section{$\underline{\text { List of Tables }}$}

Table 3.1 Comparison of main parameters of the tetrahedral $\mathrm{Au}_{20}$ neutral cluster 45 after the geometrical structure optimization.

Table 4.1 Energy of each initial plasmon state 59

Table 4.2 Summary of the relaxation time constants 71

$\begin{array}{lll}\text { Table 5.1 Optical parameters } & 79\end{array}$

Table 5.2 $\mathrm{Au}-\mathrm{ZnO}$ samples synthesized at different annealing temperatures and 85 different Au concentrations

Table 6.1 $\quad \mathrm{Au}-\mathrm{ZnO}$ nanopyramids synthesis parameters 126

Table 6.2 d-spacing values of $\mathrm{Au}-\mathrm{ZnO}$ interfaces 139 


\section{Chapter 1}

\section{Introduction}

\subsection{Heterogeneous photocatalysts}

Heterostructures of semiconductor metal oxides and metals have been studied extensively over the past few decades for photocatalysis due to their enhanced catalytic activity over just the semiconductor metal oxide alone. ${ }^{1-8}$ Size and shape tunable properties of metal nanoparticles will open a wide range of applications for metal-oxide heterostructures such as photovoltaics, optoelectronics, plasmonics, $\mathrm{CO}_{2}$ conversion, photodegradation of organic pollutants, photochemical water splitting, solar energy conversion, bio-medical applications, solar vapor generation and sterilization of surgical equipment. ${ }^{6,9-15}$ The heterogeneous photocatalysis is still continuously investigated in the fields of Physical Chemistry, Applied Physics, Chemical Engineering and Materials Science due to their higher demand. ${ }^{22,} 23$

The heterogeneous photocatalysis can be identified as a material which increases the rate of the photo reaction in the presence of the heterostructured catalyst. ${ }^{22}$ The discovery of photoelectrochemical water splitting in to hydrogen and oxygen in 1972 by Fujishima and Honda ${ }^{24}$ using a n-type $\mathrm{TiO}_{2}$ as a photoanode irradiated with UV inspired many researches to focus on heterogeneous photocatalysis. Wide band gap semiconductor metal oxides such as $\mathrm{TiO}_{2}$ and $\mathrm{ZnO}$ are among the two materials most widely investigated semiconductor metal oxide based photocatalysts due to their low cost, long term photo stability and nontoxicity ${ }^{25}$ However, poor optical activity, rapid electron-hole recombination after the photoexcitation, low efficiency of 
carrier separation and unfavorable band alignment for photo-reactions can be identified as major problems with these wide band gap semiconductor metal oxide photocatalysts. ${ }^{26-28}$ One of the main goals of this dissertation work is, addressing the above mentioned major problems in metalsemiconductor metal oxide photocatalysts. Heterostructures of semiconductor metal oxides and metals such as $\mathrm{Au}-\mathrm{ZnO}, \mathrm{Ag}-\mathrm{ZnO}, \mathrm{Au}-\mathrm{TiO}_{2}$ and $\mathrm{Ag}-\mathrm{TiO}_{2}$ can be identified as one of the solutions for above mentioned problems. ${ }^{1,4,8,29-32}$ These heterostructured photocatalysts usually show higher photocatalytic activity over the oxide itself due to improved charge separation mechanism, plasmonic heating and field enhancement created by surface plasmon resonance of metal. ${ }^{12,15,22,}$ ${ }^{33}$ Since noble metal particle plays a main role in the catalysis process, it can be identified as a metal co-catalyst. ${ }^{22}$

\subsection{Au-ZnO heterostructures}

$\mathrm{ZnO}$ is a wide band gap (3.3 eV) semiconductor metal oxide with high exciton binding energy (60 $\mathrm{meV})$ and high electron mobility at room temperature $\left(157 \mathrm{~cm}^{2} \mathrm{v}^{-1} \mathrm{~s}^{-1}\right){ }^{13,34-36}$ Many researchers attempted to overcome the major problems of the catalytic efficiency of $\mathrm{ZnO}$ which were mentioned earlier (mainly poor optical activity and electron-hole recombination). ${ }^{37-42}$ In fact, heterostructues of $\mathrm{Au}$ and $\mathrm{ZnO}$ are one of the most widely studied photoactive materials due to the higher charge separation efficiency and enhanced photocatalytic activity imparted to the $\mathrm{ZnO}$ by the addition of $\mathrm{Au} .{ }^{2-4,6}$ As such, $\mathrm{Au}-\mathrm{ZnO}$ heterostructures can be used for $\mathrm{CO}_{2}$ conversion, solar energy conversion to electricity, photodegradation of organic pollutants, and photochemical water splitting. ${ }^{6,12-15}$ Due to the large band gap of $3.3 \mathrm{eV}(\lambda \sim 375 \mathrm{~nm}), \mathrm{ZnO}$ can only absorb UV radiation which is $4-5 \%$ of the solar spectrum (Figure 1.1). ${ }^{36}$ Therefore, it is still challenging to utilize the solar spectrum effectively with $\mathrm{ZnO}$ based catalysts. The surface plasmon resonance of $\mathrm{Au}$ 
nanoparticles in $\mathrm{Au}-\mathrm{ZnO}$ heterostructures enable it to access the visible range of solar spectrum. Figure 1.2 shows the diffuse reflectance spectra for pure $\mathrm{ZnO}$ and $\mathrm{Au}-\mathrm{ZnO}$ heterostructures. ${ }^{12}$ The broad absorption peak between $500-600 \mathrm{~nm}$ is due to the surface plasmon resonance of $\mathrm{Au}$ nanoparticles.

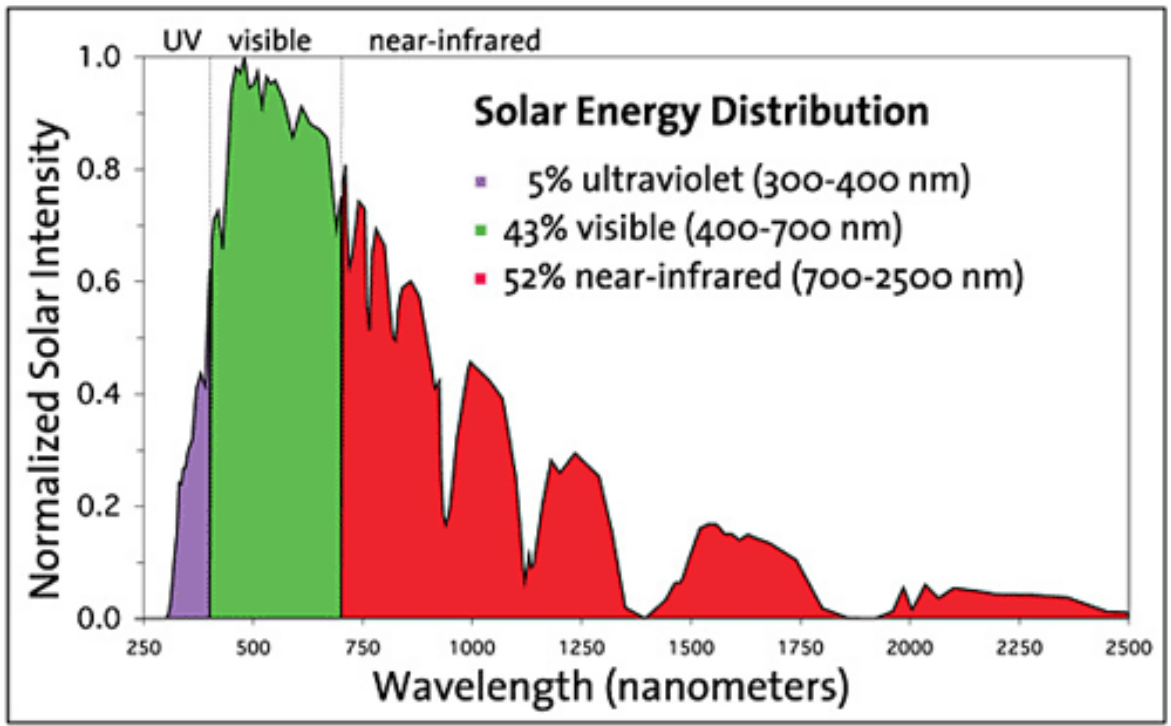

Figure 1.1 Comparison of solar intensity as function of wavelength ${ }^{16}$

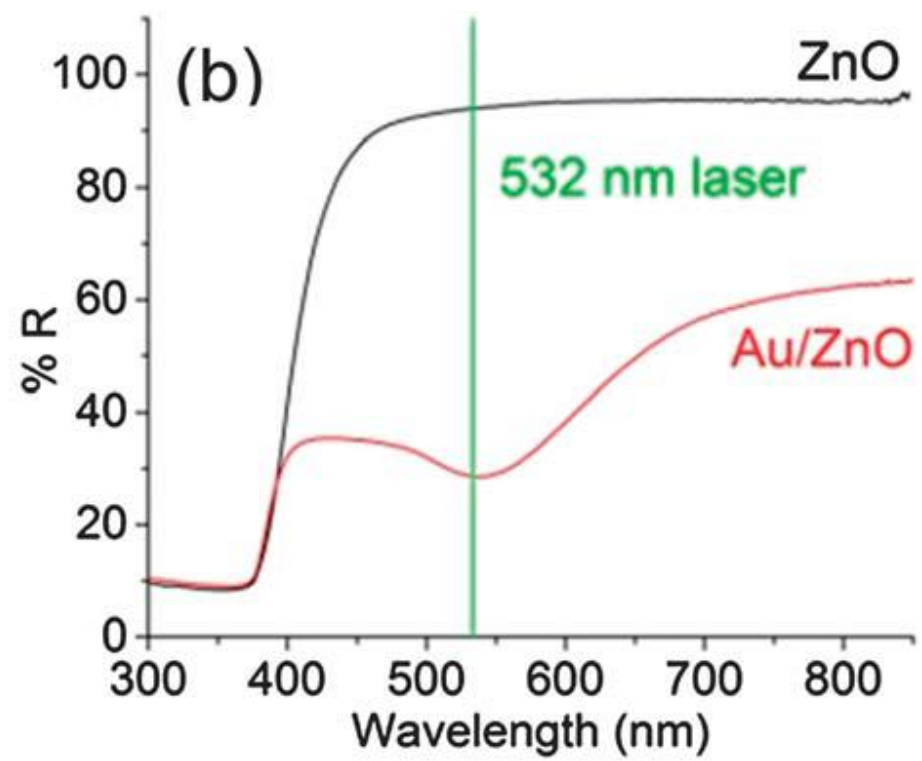

Figure 1.2 Comparison of diffuse reflectance spectra of pure $\mathrm{ZnO}$ and $\mathrm{Au}-\mathrm{ZnO}^{12}$ 


\subsubsection{Surface plasmons}

Optical properties of metallic nanoparticles have a very long history and beautifully colored stain glass windows in historic chapels is an evidence for this. However, the first scientific observation of surface plasmons was done by Robert W. Wood in $1902 .{ }^{43}$ In 1904 James Clerk Maxwell Garnett explained the bright colors observed in metal doped glasses using the Drude theory of metals with the help of electromagnetic properties of small spheres derived by Lord Rayleigh. ${ }^{44-46}$ Then in 1908, Gustav Mie introduced the famous theory of light scattering by small spherical particles. ${ }^{47}$ In 1956, David Pines theoretically explained the characteristic energy losses experienced by fast electrons travelling through metals and he called these oscillations as "plasmons". ${ }^{48}$ In the same year, Robert Fano introduced the term "polaritons" for the coupled oscillations of bound electrons and light inside transparent media. ${ }^{45,49,50}$ In 1957, Rufus Ritchie studied electron energy losses in thin films and published the first theoretical description of surface plasmons. ${ }^{51}$ Then in 1968, Ritchie explained the anomalous behavior of metal gratings in terms of surface plasmon resonance excited on the gratings. ${ }^{52}$ In the same year, Otto and Kretschmann proposed methods for the optical excitation of surface plasmons on metallic films. ${ }^{53,54}$ In 1970 , for the first time Kreibig and Zacharias explained the optical properties of metal nanoparticles in terms of surface plasmons and they compared the responses of optical and electronic properties of gold and silver nanoparticles. ${ }^{55}$ After that, research based on plasmon resonance was rapidly expanded due to the wide range of practical applications like surface enhanced Raman scattering (SERS), data storage, solar cells and sensors etc. ${ }^{45,50,56}$

Surface plasmons can be identified as collective oscillations of free electrons in the interface between metals and dielectrics. ${ }^{45,57}$ Generally, surface plasmons are divided in to propagating 
surface plasmons and localized surface plasmons. ${ }^{56,58}$ Propagating surface plasmons can be excited in metallic films and localized surface plasmons can be excited in metallic nanoparticles. ${ }^{56}$ Surface plasmons are responsible for strong enhancement of local electromagnetic field due to the strong interaction with light. ${ }^{57}$ Oscillations of the free electrons on metals that are responsible for localized surface plasmons are confined to the surface of the metallic nanoparticles and strongly interact with light. When irradiated with light at the plasmon resonance frequency of metallic nanoparticles, they generate an intense local electromagnetic field at the surface of those metallic nanoparticles. ${ }^{57}$ The plasmon resonance frequency depends on the material of the nanoparticles, surrounding medium, size of the nanoparticles and shape of the nanoparticles. ${ }^{57}$ Figure 1.3 shows the field distribution at metal-dielectric interface and the decay of electric field strength in the direction of $\mathrm{Z}$ axis. ${ }^{59}$

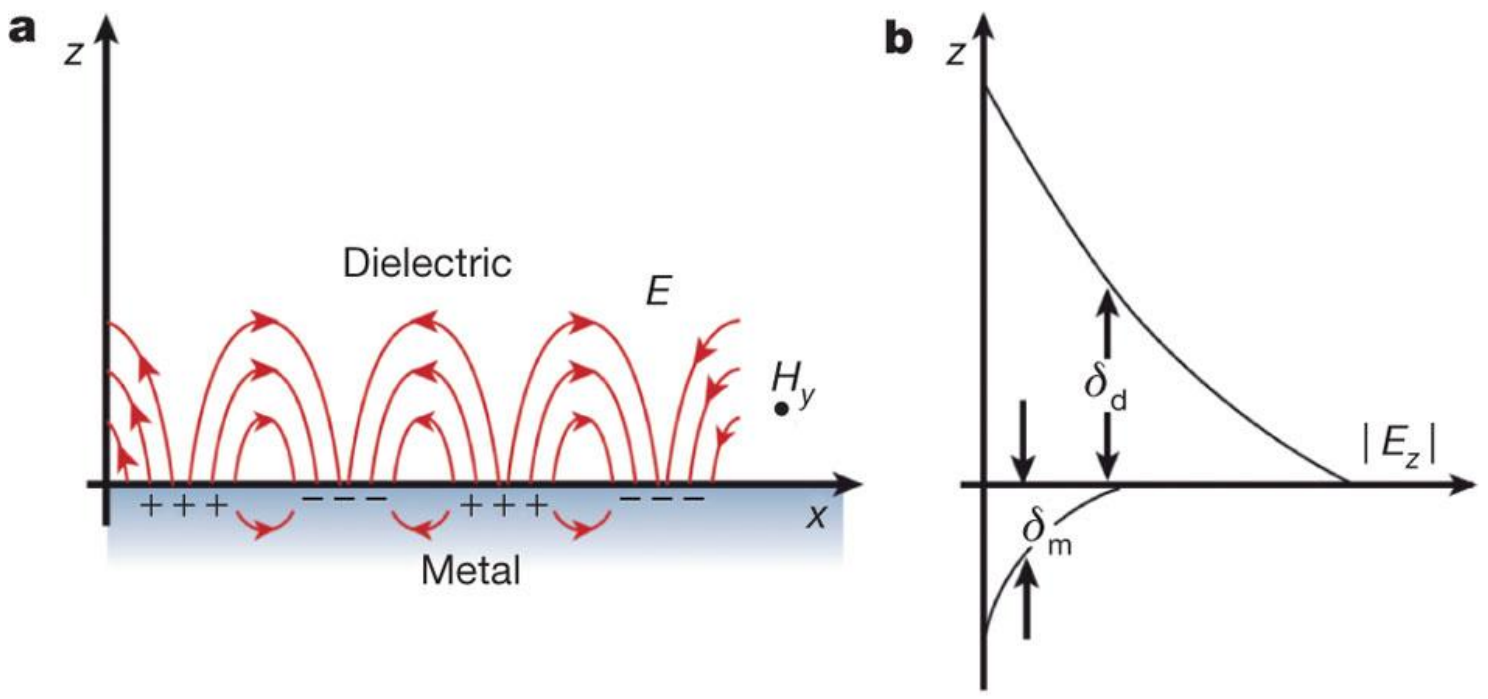

Figure 1.3 (a) The field distributions of electron charge density fluctuations in metal-dielectric interface (b) The exponential decay of the electric filed in the direction $\mathrm{Z}$ at the metal-dielectric interface with the characteristic lengths ${ }^{19}$ 
Figure 1.4 shows the absorption, scattering and extinction spectra of different shapes and sizes of Ag nanoparticles. A similar trend can be observed for Au nanoparticles. ${ }^{19}$ This allows to tune the surface plasmon resonance frequency of noble metal particles in a wide range.
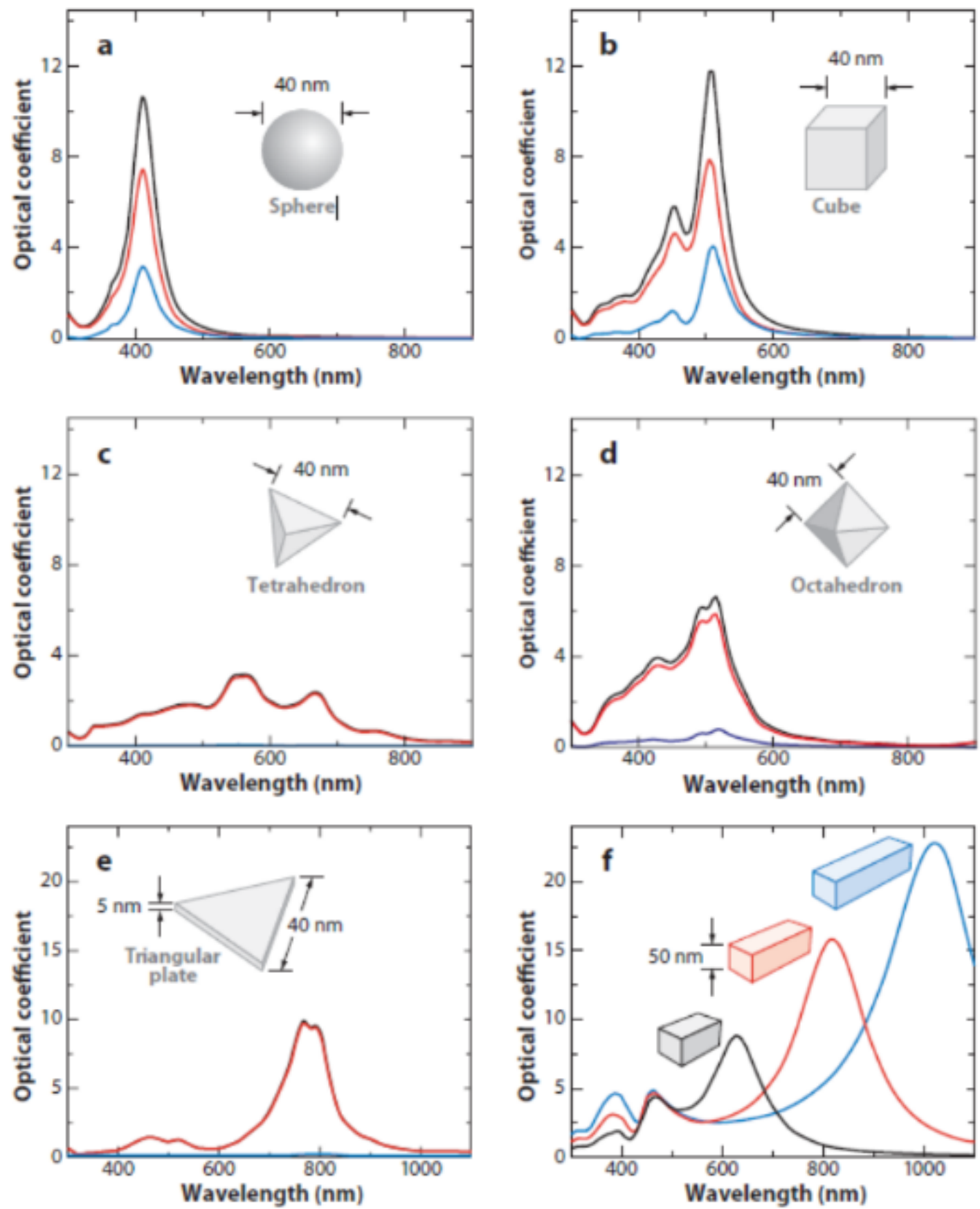

Figure 1.4 Calculated extinction (black), absorption (red), and scattering (blue) spectra for Ag nanoparticles of different shapes: (a) sphere (b) cube (c) tetrahedron (d) octahedron and (e) triangular plate. ${ }^{18}$ (f) Calculated extinction spectra of rectangular Ag nano bars with different aspect ratios of 2 (black), 3 (red), and 4 (blue). ${ }^{21}$ 


\subsubsection{Surface plasmons induced hot electron assisted photocatalytic activity of Au-ZnO}

The hot electron injection from plasmonic $\mathrm{Au}$ nanoparticles to $\mathrm{ZnO}$ (semiconductor metal oxide) was reported by several previous work. ${ }^{2,5,20,60}$ However, hot electron injection from metal to semiconductor is still inefficient due to the energy loss by ultrafast relaxation of hot electrons to lower energy level via electron-electron and electron-phonon scattering. ${ }^{61-63}$ In these metal semiconductor heterostructured catalysts, there is a Schottky barrier which is formed at the metal - semiconductor interface. ${ }^{42,64,65}$ In general, Schottky barrier forms when two materials with different work functions are in contact. The work function of $\mathrm{ZnO}$ and that of $\mathrm{Au}$ are $5.3 \mathrm{eV}$ and $5.1 \mathrm{eV}$ respectively. ${ }^{66,67}$ Since Au has a lower work function compared to the $\mathrm{ZnO}$, during the formation of the Schottky barrier electrons flow from $\mathrm{Au}$ to $\mathrm{ZnO}$ in order to equilibrate the Fermi level. $^{60}$

This Schottky barrier inhibits the rapid recombination of electron-hole pairs. ${ }^{30}$ Since new Fermi energy level is below the conduction band of the semiconductor, photo excited electrons can transfer from semiconductor to the metal which avoids the recombination of photoexcited electrons and holes. ${ }^{30}$ However, hot electrons should have an energy higher than the Schottky barrier in order to overcome the barrier. ${ }^{68}$ Figure 1.5 shows a schematic diagram for the organic dye degradation using the $\mathrm{Au}-\mathrm{ZnO}$ heterostructures illuminated by visible light. ${ }^{60}$ Plasmon induced hot electrons are injected in to the conduction band of the $\mathrm{ZnO}$ from $\mathrm{Au}$. Here, the role of $\mathrm{Au}$ is visible light harvesting and facilitating the charge transfer process. ${ }^{20}$ Injected electrons in conduction band react with adsorbed $\mathrm{O}_{2}$ to generate superoxide radicals $\left(\mathrm{O}_{2}{ }^{--}\right)$. Then, superoxide radicals generate hydroxyl radicals $\left(\mathrm{OH}^{*}\right)$ with water. Also, $\mathrm{Yu}$ et $a l^{20}$ reported that holes in $\mathrm{Au}$ 
also generate the $\mathrm{OH}^{*}$ radicals. Both radicals are responsible for degradation of organic dye molecules. $^{60}$

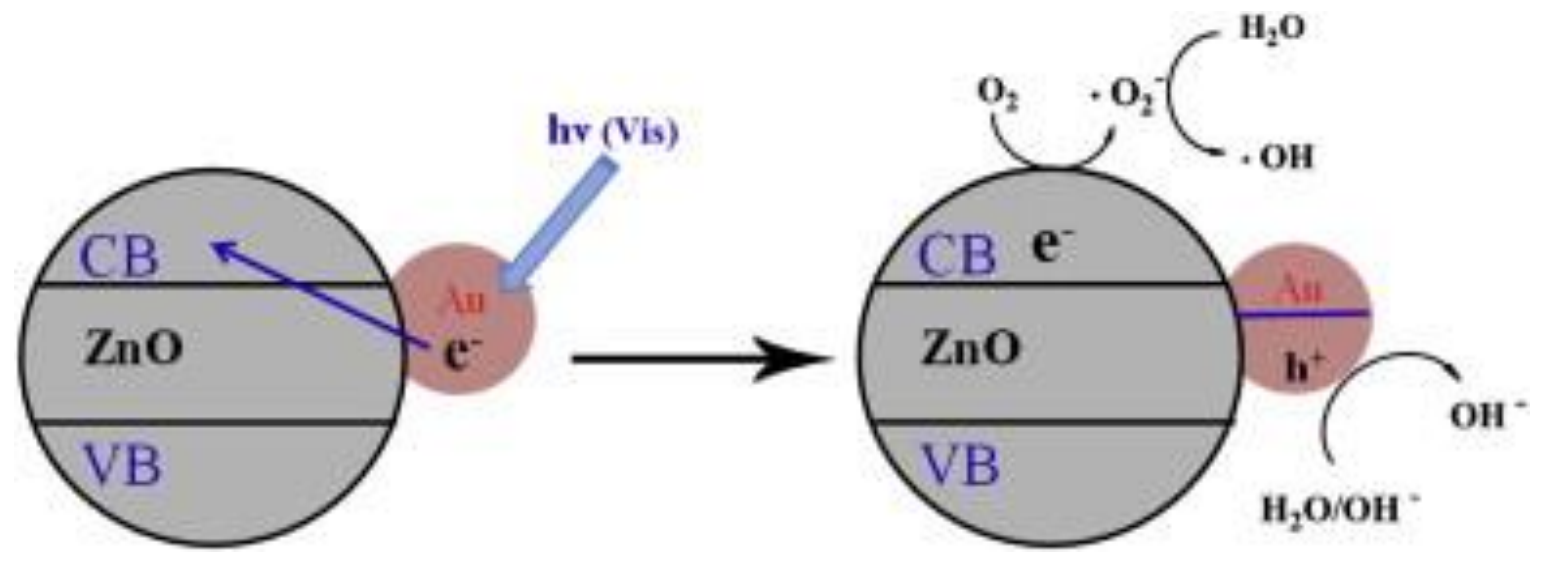

Figure 1.5 Proposed mechanism for the photodegradation of organic dyes with $\mathrm{Au}-\mathrm{ZnO}$ heterostructures under visible light illumination. ${ }^{20}$

\subsubsection{Photocatalytic activity of Au-ZnO under UV illumination}

The photocatalytic activity of $\mathrm{Au}-\mathrm{ZnO}$ heterostructures is more efficient under the UV illumination compared to the visible light illumination. ${ }^{20}$ Under UV illumination, hot electron generation is very efficient due to the band gap excitation of $\mathrm{ZnO}$. Photodegradation of organic dyes using the $\mathrm{Au}-\mathrm{ZnO}$ under UV illumination was reported in several previous work. 1, 4, 6, 8, 20, 69-71 The photodegradation mechanism under UV illumination is slightly different compared to visible light illumination. Under UV illumination, it is possible to excite the band gap of $\mathrm{ZnO}$ as well as surface plasmons of $\mathrm{Au}$ in $\mathrm{Au}-\mathrm{ZnO}$ heterostructures. Therefore, both mechanisms might be responsible for generation of reactive oxygen species under broadband illumination. Since both mechanisms generate the same reactive oxygen species, it is difficult to evaluate the efficiency of each 
mechanism using broadband or white light illumination. However, it is possible to evaluate the efficiency of each mechanism separately when using the long pass filters (more details discussed in Chapter 6). He et $a l^{71}$ identified the reactive oxygen species generated from $\mathrm{Au}-\mathrm{ZnO}$ under simulated sunlight illumination. Figure 1.6 shows the proposed mechanism by Fageria et al ${ }^{60}$ for photodegradation mechanism of organic dyes using $\mathrm{Au}-\mathrm{ZnO}$ heterostructures under UV illumination.

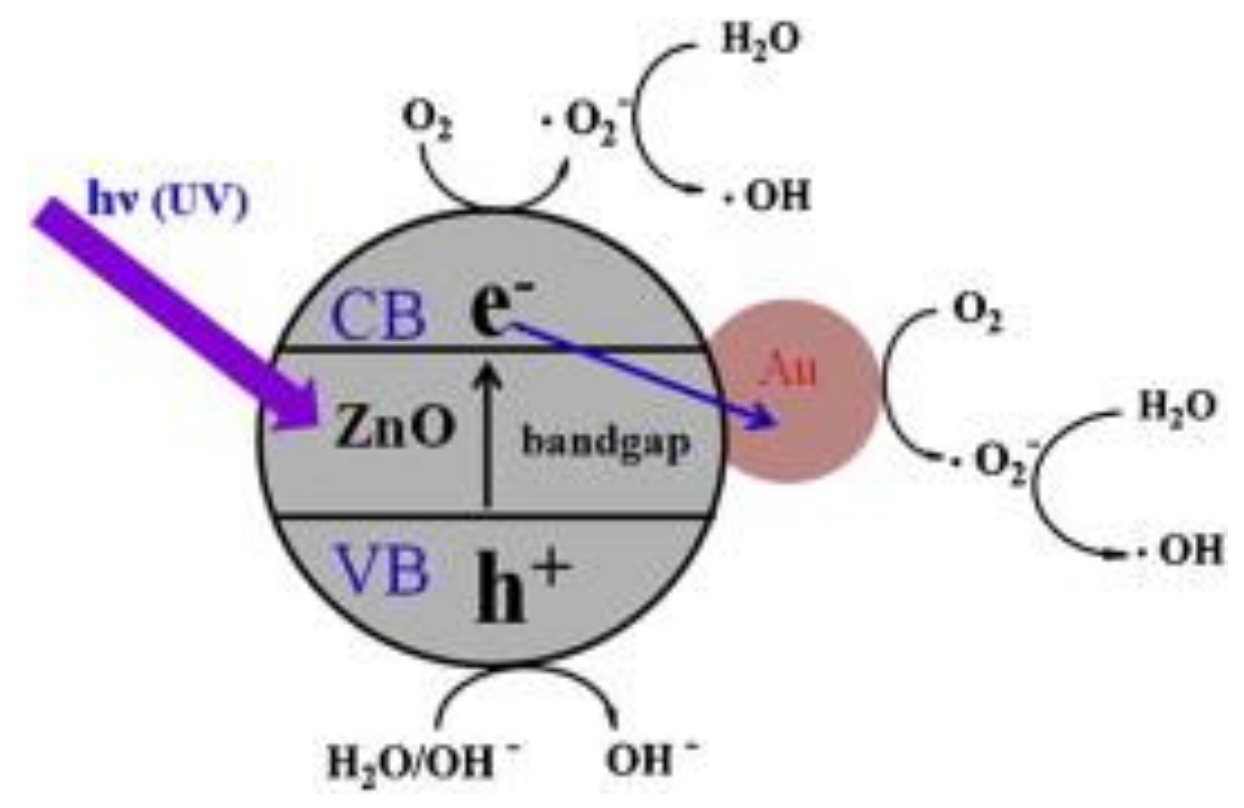

Figure 1.6 Proposed mechanism for the photodegradation of organic dyes with $\mathrm{Au}-\mathrm{ZnO}$ heterostructures under UV light illumination. ${ }^{20}$

Under UV (energy greater than the band gap $=3.3 \mathrm{eV}$ ) illumination, photoexcited electrons in the conduction band of $\mathrm{ZnO}$ will be injected in to the $\mathrm{Au}$ while holes are created at the valence band of the $\mathrm{ZnO} .{ }^{20}$ In this mechanism, the role of $\mathrm{Au}$ is very important since $\mathrm{Au}$ acts as an electron 
reservoir to avoid the quick electron-hole recombination in $\mathrm{ZnO}$. Hydroxyl radicals are generated at the valence band of the $\mathrm{ZnO}$ in addition to the conduction band of $\mathrm{ZnO}$ and surface of the $\mathrm{Au} .^{20}$

\subsection{Plasmonic heat generation}

Noble metal nanoparticles have an extremely large absorption cross section at their plasmon wavelength. ${ }^{72}$ In other words, metal nanoparticles can absorb a large amount of light and almost completely convert it to heat at the surface plasmon resonance frequency. ${ }^{72}$ Plasmonic heat generation from the metal nanoparticles have been already reported by several previous work. ${ }^{72-78}$ Due to the possibility of synthetically tuning the surface plasmon resonance frequency in visible and near infrared range, these metal nanoparticles are suitable candidates for laser induced drug release, bio imaging, thermally driven photocatalysis, steam generation, sterilization, waterethanol distillation and developing sensors. ${ }^{10,12,77,79}$ When the nanoparticles are illuminated by an electromagnetic field, a portion of the electromagnetic field is scattered and the rest is absorbed by the nanoparticles. ${ }^{80}$

The scattered light does not contribute to the plasmonic heating and only the absorbed light generates heat. After the light absorption by nanoparticles, the electromagnetic field at the interface of the metal-dielectric drastically increases. This very high electromagnetic field is highly localized and the generated heat is transferred in to the vicinity of few nanometers. In other words, it is possible to heat up the highly localized area surrounded by the Au nanoparticles in $\mathrm{Au}-\mathrm{ZnO}$ heterostructures without increasing the temperature of the bulk sample. The amount of heat generation depends on various factors such as material of the nanoparticles, size of the nanoparticles, geometrical shape of the nanoparticles, surrounding medium and the intensity of the 
illuminating electromagnetic radiation. ${ }^{17}$ Figure 1.7 shows calculated heat generation for different shapes of Au nanoparticles. ${ }^{17}$ When the aspect ratio were changed from spheres to tubes, the maximum heat generation peak was red shifted and it can be attributed to the red shifting of the wavelength of plasmon resonance. Govorov et al ${ }^{81}$ used simplified Mie theory in order to calculate the temperature at the surface of spherical shaped Au nanoparticles. Chapter 5 discusses the plasmonic heat assisted photocatalytic activity of $\mathrm{Au}-\mathrm{ZnO}$ heterostructures in detail.

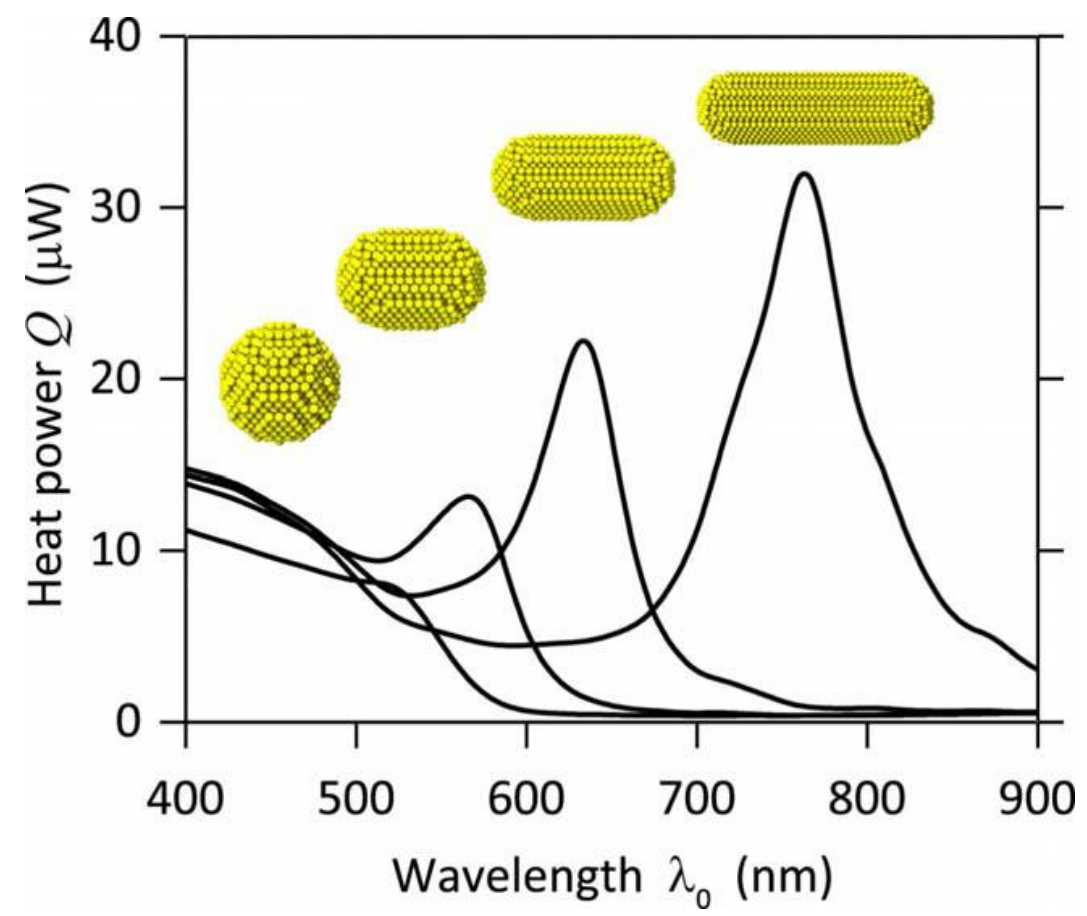

Figure 1.7 Calculated heat generation spectra for different sizes of Au nanoparticles. Aspect ratios starting from sphere 1:1, 1.4:1, 2:1, and 3:1. ${ }^{17}$ 


\subsubsection{Importance of $\mathrm{ZnO}$ for plasmonic heating assisted photocatalysis}

$\mathrm{ZnO}$ based catalysts such as $\mathrm{Cu}-\mathrm{ZnO}$ are a good candidate for methanol synthesis from $\mathrm{CO}_{2}$ using water-gas shift reactions. However, the reactions should be carried out at higher temperatures in order to achieve maximum efficiency. ${ }^{82,83}$ Since increasing the temperature of $\mathrm{ZnO}$ using an energy source which generates $\mathrm{CO}_{2}$ is not worthwhile, plasmonic heating was used to increase the temperature of $\mathrm{ZnO}$ in this work (see Chapter 5 for more details). Since plasmonic heat generation is a highly localized process in nanoscale, still there is no method to directly measure the local temperature. The temperature dependent behavior of some Raman modes of $\mathrm{ZnO}$ such as $E_{2}($ high $)$ can be used to investigate the local temperature of $\mathrm{ZnO}{ }^{84-87}$ Since $\mathrm{ZnO}$ has good catalytic activity for $\mathrm{CO}_{2}$ conversion and possibility to investigate the local temperature using temperature dependent Raman spectra, $\mathrm{ZnO}$ was identified as a good candidate for making heterostructures with $\mathrm{Au}$ for plasmonic heating-assisted $\mathrm{CO}_{2}$ conversion.

\subsection{Importance of $\mathrm{CO}_{2}$ conversion}

The energy demand of the United States as well as in the world increases every year with increasing population and the growth of the economy. ${ }^{12}$ According to statistics, fossil fuels will supply $80 \%$ of the world energy demand for at least next few decays. ${ }^{88}$ Burning of fossil fuels release tons of $\mathrm{CO}_{2}$ in to the environment. $\mathrm{CO}_{2}$ is a greenhouse gas and excess $\mathrm{CO}_{2}$ mainly results in increasing the earth's temperature. As a result of increased earth's temperature, a variety of critical problems can be expected such as melting polar ice and rising sea level, undesirable effects on agriculture, extreme weather conditions and spread of diseases. The main natural $\mathrm{CO}_{2}$ removal method is the photosynthesis by plants. However, increasing deforestation results in inefficient 
photosynthesis. The natural option is unable to handle huge amounts of $\mathrm{CO}_{2}$ outputs from the world fossil fuel consumption. Therefore, atmospheric $\mathrm{CO}_{2}$ concentration increases each year. The atmospheric $\mathrm{CO}_{2}$ concentration was $\sim 280 \mathrm{ppm}$ before the industrial revolution and it is expected to reach $\sim 570 \mathrm{ppm}$ at end of this century. ${ }^{89}$

\subsection{Computational calculations}

It is very important to understand the properties of the materials using computational calculations. Even though it is difficult to model the actual experimental work, it will help to explore more insight of the materials. In this work, computational calculations were performed in order to calculate vibrational modes, phonon spectral density and plasmon relaxation time constants. Two $\mathrm{Au}$ clusters $\left(\mathrm{Au}_{20}\right.$ and $\left.\mathrm{Au}_{55}\right)$ which contain 20 and 55 of $\mathrm{Au}$ atoms respectively were used for the calculations. The diameter of $\mathrm{Au}_{55}$ is around $\sim 1.4 \mathrm{~nm}$ and it is not possible to use larger $\mathrm{Au}$ nanoparticles due to long simulation times and necessity of the high computational power.

The eigen vectors of vibrational modes of the $\mathrm{Au}_{20}$ cluster were used to calculate the phonon spectral density. $\mathrm{Au}_{55}$ has a moderate size and the Density Functional Theory (DFT) calculations on it gives more reliable data which can be compared with experimental data. It was used to investigate the plasmon relaxation dynamics. For all the DFT calculations, BLYP (Becke's 1988 exchange functional with the correlation functional by Lee, Yang, and Parr) method and SNP (Single Numerical basis set including d-polarization function) basis were used to describe the electronic wave function. 


\subsection{Structure of the dissertation}

Chapter 2 explains the brief theory behind each of the various type of experimental technique which were utilized for material characterization. Chapter 3 explains the computational work for vibrational modes and phonon spectral density calculations for the $\mathrm{Au}_{20}$ cluster. Plasmon relaxation dynamics of $\mathrm{Au}_{55}$ is explained in Chapter 4. Chapter 5 consists of all the details of synthesis and characterization of $\mathrm{Au}-\mathrm{ZnO}$ heterostructures, plasmonic heating assisted $\mathrm{CO}_{2}$ conversion and local temperature estimation using the temperature dependent phonon model. Chapter 6 discusses the study of $\mathrm{Au}-\mathrm{ZnO}$ heterostructures as a photocatalyst for waste water remediation. 


\section{References}

1. K. Flomin, I. Jen-La Plante, B. Moshofsky, M. Diab and T. Mokari, Nanoscale, 2014, 6, 1335-1339.

2. Z. Han, L. Wei, Z. Zhang, X. Zhang, H. Pan and J. Chen, Plasmonics, 2013, 8, 11931202.

3. W. He, H.-K. Kim, W. G. Wamer, D. Melka, J. H. Callahan and J.-J. Yin, Journal of the American Chemical Society, 2013, 136, 750-757.

4. P. Li, Z. Wei, T. Wu, Q. Peng and Y. Li, Journal of the American Chemical Society, $2011,133,5660-5663$.

5. M. Misra, P. Kapur and M. L. Singla, Applied Catalysis B: Environmental, 2014, 150_ 151, 605-611.

6. C. Mondal, J. Pal, M. Ganguly, A. K. Sinha, J. Jana and T. Pal, New Journal of Chemistry, 2014, 38, 2999-3005.

7. Q. Wang, B. Geng and S. Wang, Environmental Science \& Technology, 2009, 43, 89688973.

8. K. X. Yao, X. Liu, L. Zhao, H. C. Zeng and Y. Han, Nanoscale, 2011, 3, 4195-4200.

9. P. V. Kamat, The Journal of Physical Chemistry C, 2007, 111, 2834-2860.

10. O. Neumann, A. S. Urban, J. Day, S. Lal, P. Nordlander and N. J. Halas, ACS Nano, 2013, 7, 42-49.

11. J. Zhao, J. A. Bardecker, A. M. Munro, M. S. Liu, Y. Niu, I. K. Ding, J. Luo, B. Chen, A. K. Y. Jen and D. S. Ginger, Nano Letters, 2006, 6, 463-467.

12. C. Wang, O. Ranasingha, S. Natesakhawat, P. R. Ohodnicki, M. Andio, J. P. Lewis and C. Matranga, Nanoscale, 2013, 5, 6968-6974. 
13. V. Dhas, S. Muduli, W. Lee, S.-H. Han and S. Ogale, Applied Physics Letters, 2008, 93, 243108.

14. H. M. Chen, C. K. Chen, C.-J. Chen, L.-C. Cheng, P. C. Wu, B. H. Cheng, Y. Z. Ho, M. L. Tseng, Y.-Y. Hsu, T.-S. Chan, J.-F. Lee, R.-S. Liu and D. P. Tsai, ACS Nano, 2012, 6, 7362-7372.

15. S. K. Cushing and N. Wu, in Interface, The Electrochemical Society, 2013, pp. 63-67.

16. K. Biernat, A. Malinowski and M. Gnat, ed. Z. Fang, 2013.

17. G. Baffou, R. Quidant and C. Girard, Applied Physics Letters, 2009, 94, 153109.

18. X. Lu, M. Rycenga, S. E. Skrabalak, B. Wiley and Y. Xia, Annual Review of Physical Chemistry, 2009, 60, 167-192.

19. B. J. Wiley, S. H. Im, Z.-Y. Li, J. McLellan, A. Siekkinen and Y. Xia, The Journal of Physical Chemistry B, 2006, 110, 15666-15675.

20. H. Yu, H. Ming, H. Zhang, H. Li, K. Pan, Y. Liu, F. Wang, J. Gong and Z. Kang, Materials Chemistry and Physics, 2012, 137, 113-117.

21. B. J. Wiley, Y. Chen, J. M. McLellan, Y. Xiong, Z.-Y. Li, D. Ginger and Y. Xia, Nano Letters, 2007, 7, 1032-1036.

22. A. Ibhadon and P. Fitzpatrick, Catalysts, 2013, 3, 189-218.

23. W. Y. Teoh, J. A. Scott and R. Amal, The Journal of Physical Chemistry Letters, 2012, 3, 629-639.

24. A. Fujishima and K. Honda, Nature, 1972, 238, 37-38.

25. J. Schneider, M. Matsuoka, M. Takeuchi, J. Zhang, Y. Horiuchi, M. Anpo and D. W. Bahnemann, Chemical Reviews, 2014, 114, 9919-9986. 
26. A. J. Cowan, W. Leng, P. R. F. Barnes, D. R. Klug and J. R. Durrant, Physical Chemistry Chemical Physics, 2013, 15, 8772-8778.

27. X. Zhang, J. Qin, Y. Xue, P. Yu, B. Zhang, L. Wang and R. Liu, Sci. Rep., 2014, 4.

28. R. Long, N. J. English and O. V. Prezhdo, The Journal of Physical Chemistry Letters, 2014, 5, 2941-2946.

29. Y. Zheng, L. Zheng, Y. Zhan, X. Lin, Q. Zheng and K. Wei, Inorganic Chemistry, 2007, 46, 6980-6986.

30. F. Sun, F. Tan, W. Wang, X. Qiao and X. Qiu, Materials Research Bulletin, 2012, 47, 3357-3361.

31. Y. Wen, H. Ding and Y. Shan, Nanoscale, 2011, 3, 4411-4417.

32. H. Wang, T. You, W. Shi, J. Li and L. Guo, The Journal of Physical Chemistry C, 2012, 116, 6490-6494.

33. W. Hou, W. H. Hung, P. Pavaskar, A. Goeppert, M. Aykol and S. B. Cronin, ACS Catalysis, 2011, 1, 929-936.

34. R. C. Pawar, D. Cho and C. S. Lee, Current Applied Physics, 2013, 13, Supplement 2, S50-S57.

35. R. C. Pawar, J. S. Shaikh, P. S. Shinde and P. S. Patil, Materials Letters, 2011, 65, 22352237.

36. X. Zhang, J. Qin, Y. Xue, P. Yu, B. Zhang, L. Wang and R. Liu, Scientific Reports, 2014, 4, 4596 .

37. M. E. Aguirre, G. Perelstein, A. Feldhoff, A. Condo, A. J. Tolley and M. A. Grela, New Journal of Chemistry, 2015, 39, 909-914. 
38. H. Yu, H. A. I. Ming, J. Gong, H. Li, H. U. I. Huang, K. Pan, Y. Liu, Z. Kang, J. I. E. Wei and D. Wang, Bull Mater Sci, 2013, 36, 367-372.

39. J. W. Chiou, S. C. Ray, H. M. Tsai, C. W. Pao, F. Z. Chien, W. F. Pong, M.-H. Tsai, J. J. Wu, C. H. Tseng, C.-H. Chen, J. F. Lee and J.-H. Guo, Applied Physics Letters, 2007, 90, 192112.

40. T. Wang, B. Jin, Z. Jiao, G. Lu, J. Ye and Y. Bi, Journal of Materials Chemistry A, 2014, 2, 15553-15559.

41. N. Gogurla, A. K. Sinha, S. Santra, S. Manna and S. K. Ray, Sci. Rep., 2014, 4.

42. T. Bora, H. H. Kyaw, S. Sarkar, S. K. Pal and J. Dutta, Beilstein Journal of Nanotechnology, 2011, 2, 681-690.

43. $\quad$ R. W. Wood, in Phil. Mag., 1902.

44. J. C. M. Garnett, Colours in Metal Glasses and in Metallic Films, 1904.

45. P. G. Kik, Surface Plasmon Nanophotonics, Springer Netherlands, 2007.

46. A. Kulkarni, D. Guney and A. Vora, ISRN Nanomaterials, 2013, 2013, 7.

47. G. Mie, Annalen der Physik, 1908, 330, 377-445.

48. D. Pines, Reviews of Modern Physics, 1956, 28, 184-198.

49. U. Fano, Physical Review, 1956, 103, 1202-1218.

50. Z. Junxi, Z. Lide and X. Wei, Journal of Physics D: Applied Physics, 2012, 45, 113001.

51. R. H. Ritchie, Physical Review, 1957, 106, 874-881.

52. R. H. Ritchie, E. T. Arakawa, J. J. Cowan and R. N. Hamm, Physical Review Letters, 1968, 21, 1530-1533.

53. A. Otto, Z. Physik, 1968, 216, 398-410.

54. E. Kretschmann and H. Raether, Z. Naturforsch. A, 1968, 23, 2135. 
55. U. Kreibig and P. Zacharias, Z. Physik, 1970, 231, 128-143.

56. Y. Chen and H. Ming, Photonic Sens, 2012, 2, 37-49.

57. W. Hou and S. B. Cronin, Advanced Functional Materials, 2013, 23, 1612-1619.

58. S. A. Maier, Plasmonics: Fundamentals and Applications, Springer US, 2007.

59. E. S. Kryachko and F. Remacle, International Journal of Quantum Chemistry, 2007, 107, 2922-2934.

60. P. Fageria, S. Gangopadhyay and S. Pande, RSC Advances, 2014, 4, 24962-24972.

61. J. H. Hodak, I. Martini and G. V. Hartland, The Journal of Physical Chemistry B, 1998, 102, 6958-6967.

62. T. Hertel, E. Knoesel, M. Wolf and G. Ertl, Physical Review Letters, 1996, 76, 535-538.

63. W. S. Fann, R. Storz, H. W. K. Tom and J. Bokor, Physical Review B, 1992, 46, 1359213595.

64. W. I. Park, G.-C. Yi, J.-W. Kim and S.-M. Park, Applied Physics Letters, 2003, 82, 43584360.

65. A. Y. Polyakov, N. B. Smirnov, E. A. Kozhukhova, V. I. Vdovin, K. Ip, Y. W. Heo, D. P. Norton and S. J. Pearton, Applied Physics Letters, 2003, 83, 1575-1577.

66. Z. Zhang, G. Meng, Q. Xu, Y. Hu, Q. Wu and Z. Hu, The Journal of Physical Chemistry C, 2009, 114, 189-193.

67. X. Wang, C. J. Summers and Z. L. Wang, Applied Physics Letters, 2005, 86, 013111.

68. C. Clavero, Nat Photon, 2014, 8, 95-103.

69. N. Udawatte, M. Lee, J. Kim and D. Lee, ACS Applied Materials \& Interfaces, 2011, 3, $4531-4538$. 
70. J. Lee, H. S. Shim, M. Lee, J. K. Song and D. Lee, The Journal of Physical Chemistry Letters, 2011, 2, 2840-2845.

71. W. He, H.-K. Kim, W. G. Wamer, D. Melka, J. H. Callahan and J.-J. Yin, Journal of the American Chemical Society, 2014, 136, 750-757.

72. C. Fang, L. Shao, Y. Zhao, J. Wang and H. Wu, Advanced Materials, 2012, 24, 94-98.

73. V. K. Pustovalov, Chemical Physics, 2005, 308, 103-108.

74. A. O. Govorov and H. H. Richardson, Nano Today, 2007, 2, 30-38.

75. L. Cognet, C. Tardin, D. Boyer, D. Choquet, P. Tamarat and B. Lounis, Proceedings of the National Academy of Sciences of the United States of America, 2003, 100, 1135011355.

76. A. M. Gobin, D. P. O'Neal, D. M. Watkins, N. J. Halas, R. A. Drezek and J. L. West, Lasers in Surgery and Medicine, 2005, 37, 123-129.

77. A. G. Skirtach, C. Dejugnat, D. Braun, A. S. Susha, A. L. Rogach, W. J. Parak, H. Möhwald and G. B. Sukhorukov, Nano Letters, 2005, 5, 1371-1377.

78. C. M. Pitsillides, E. K. Joe, X. Wei, R. R. Anderson and C. P. Lin, Biophysical Journal, 2003, 84, 4023-4032.

79. D. Boyer, P. Tamarat, A. Maali, B. Lounis and M. Orrit, Science, 2002, 297, 1160-1163.

80. M. B. Cortie, X. Xu, H. Chowdhury, H. Zareie and G. Smith, 2005, pp. 565-573.

81. A. O. Govorov, W. Zhang, T. Skeini, H. Richardson, J. Lee and N. A. Kotov, Nanoscale Research Letters, 2006, 1, 84-90.

82. M. Behrens, F. Studt, I. Kasatkin, S. Kühl, M. Hävecker, F. Abild-Pedersen, S. Zander, F. Girgsdies, P. Kurr, B.-L. Kniep, M. Tovar, R. W. Fischer, J. K. Nørskov and R. Schlögl, Science, 2012, 336, 893-897. 
83. S. Natesakhawat, J. W. Lekse, J. P. Baltrus, P. R. Ohodnicki, B. H. Howard, X. Deng and C. Matranga, ACS Catalysis, 2012, 2, 1667-1676.

84. R. Cuscó, E. Alarcón-Lladó, J. Ibáñez, L. Artús, J. Jiménez, B. Wang and M. J. Callahan, Physical Review B, 2007, 75, 165202.

85. H. Kumar Yadav, R. S. Katiyar and V. Gupta, Applied Physics Letters, 2012, 100, 051906.

86. K. Samanta, P. Bhattacharya and R. S. Katiyar, Physical Review B, 2007, 75, 035208.

87. E. Alarcon-Llado, R. Cusco, J. Ibanez, L. Artus, J. Jimenez, B. Wang and M. Callahan, MRS Online Proceedings Library, 2006, 957, null-null.

88. J. Conti and P. Holtberg, U.S. Energy information Administration, Washington DC, 2011.

89. W. Wang, S. Wang, X. Ma and J. Gong, Chemical Society Reviews, 2011, 40, 3703-3727. 


\section{Chapter 2}

\section{$\underline{\text { Experimental Techniques }}$}

\subsection{Introduction}

In order to characterize the $\mathrm{Au}-\mathrm{ZnO}$ heterostructures, various experimental techniques were used. Transmission Electron Microscopy (TEM), Scanning Electron Microscopy (SEM), X-Ray Diffractrometry (XRD), Fourier Transform Infra-Red spectroscopy (FTIR), X-ray Photoelectron Spectroscopy (XPS), diffuse reflectance spectroscopy and UV-VIS absorption spectroscopy were mainly used for characterization. Gas Chromatography was used to identify the products of $\mathrm{CO}_{2}$ reduction reactions. In addition to characterization and product identification, Raman spectroscopy was used to develop an indirect method to estimate the local temperature of $\mathrm{ZnO}$ nanoparticles. Also, $\mathrm{O}_{2}$ plasma etching was used remove the excess organic molecules after the synthesis of $\mathrm{Au}-$ $\mathrm{ZnO}$ nanopyramids.

\subsection{Transmission Electron Microscopy (TEM)}

JEOL JEM-2100 Transmission Electron Microscope (TEM) equipped with a Lanthanum hexaboride $\left(\mathrm{LaB}_{6}\right)$ thermal filament was used to obtain both conventional and high resolution TEM images. Since the work function of $\mathrm{LaB}_{6}$ is lower than the work function of tungsten, $\mathrm{LaB}_{6}$ filament is more efficient than the tungsten filament. ${ }^{1}$ There are two digital Gatan cameras available in this TEM including ES500W which is capable of high speed and high quality imaging with a field of view larger than a traditional TEM film. ES500W is used to obtain conventional TEM images. SC1000 Orius is the latest generation retractable and fiber optical coupled 11 
Megapixel CCD camera which provides high quality and > 14 frames per second fast images for high resolution images.

TEM follows the same basic principle as light microscope. ${ }^{2}$ However, TEM uses a high energy electron beam as the source instead of light in the light microscope. Also, TEM uses electromagnetic lenses instead of usual glass lenses to focus the electron beam. ${ }^{3,4}$ The circular electro magnets generates a precise circular magnetic field which acts like an optical lens to focus the electron beam. ${ }^{5}$ The high energy electron beam generated from cathode goes through the specimen and some of the electrons are scattered from the atoms of the material. The electron beam is accelerated by the positive anode. ${ }^{5}$ The aperture is a thin disk with a small circular hole $(2-100 \mu \mathrm{m})$ which is used to restrict the electron beam and filter out the unwanted electrons. ${ }^{5}$ The unscattered electrons are focused in to the fluorescent screen to make a shadow image. ${ }^{2}$ The darkness of the image depends on the density of the material in that area. ${ }^{2}$ Three types of lenses can be seen in TEM. The purposes of the condenser lens and objective lens are to control the illumination of the sample and magnify the sample respectively. ${ }^{4}$ At the end of the electron beam path, the projection lens generates an amplified image. ${ }^{4}$ The most important component of the TEM is the electron gun. Also, it is necessary to maintain an Ultra-High Vacuum (UHV) to create insulation between the anode and the cathode. ${ }^{4}$ Figure 2.1 shows the schematic diagram of the TEM. ${ }^{6}$ According to Abbe's equation, the theoretical resolution of a microscope can be calculated as follows: ${ }^{4}$

$R=\frac{0.61 \lambda}{\eta(\sin \theta)}$

(Equation 2.1) 
where $R$ is the resolution of the microscope, $\lambda$ is the wavelength of the light, $\eta$ is the index of refraction of the environment surrounding the lens and $\vartheta$ is the angle of illumination. The denominator of the above equation represents the numerical aperture of the objective lens. ${ }^{4}$ According to the wave-particle duality of light, it is possible to calculate the wavelength of the electrons using the DeBroglie wave equation: $:^{4,7}$

$\lambda=\frac{h}{m v}$

where $\lambda$ is the wavelength of the accelerated electrons, $h$ is the plank's constant, $m$ is the mass of the electron and $v$ is the velocity of the electrons.

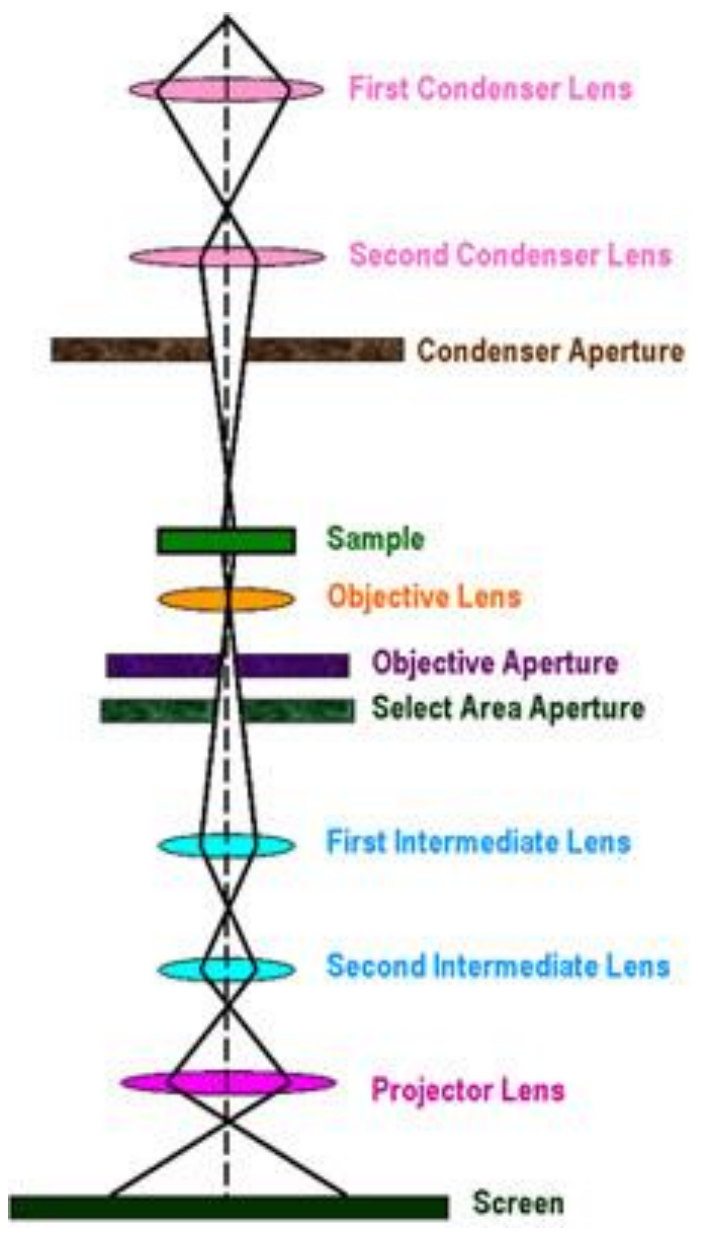

Figure 2.1 The schematic diagram of the Transmission Electron Microscope ${ }^{6}$ 
If the electron is accelerated with the potential drop $\mathrm{V}$, the wavelength of the electron can be written as,

$\lambda=\frac{h}{\sqrt{2 m_{0} V\left(1+\frac{V}{2 m_{0} c^{2}}\right)}}$

where $m_{0}$ is the rest mass of the electron. The usual operating voltage of TEM is around 100-300 $\mathrm{kV}$. Therefore, it is possible to observe the samples in atomic scale $\left(10^{-10} \mathrm{~m}\right)$. Also, when it is operated in high resolution mode (HRTEM), it is possible to observe the atomic planes which help to measure the lattice spacing of the crystal planes.

\subsection{Scanning Electron Microscopy (SEM)}

JOEL JSM-7600F Scanning Electron Microscope (SEM) is equipped with a thermal field emission gun as an electron source and was used to investigate the surface morphology of the $\mathrm{Au}-\mathrm{ZnO}$ heterostructures. Electron beam is focused using the electromagnetic lenses as same as in TEM (Figure 2.2). ${ }^{8}$ In general, the electron beam is accelerated by $200 \mathrm{~V}-40 \mathrm{kV}$ voltage and a nanometer level resolution can be achieved. ${ }^{4}$ The electron beam bombards the surface of the sample and the secondary electrons are collected while the electron beam striking position is moving smoothly. ${ }^{9}$ Two pairs of scanning coils are used to deflect the electron beam in $\mathrm{x}$ and $\mathrm{y}$ directions in order to perform the line by line scan (raster scan). The electron beam penetrate (depth of the interaction volume) around $2 \mu \mathrm{m}$ (Figure 2.3) and several types of signals can be detected after bombarding the primary electron beam in to the sample. ${ }^{4,5}$ Different types of detectors collect the output signals from the sample to generate the image. 


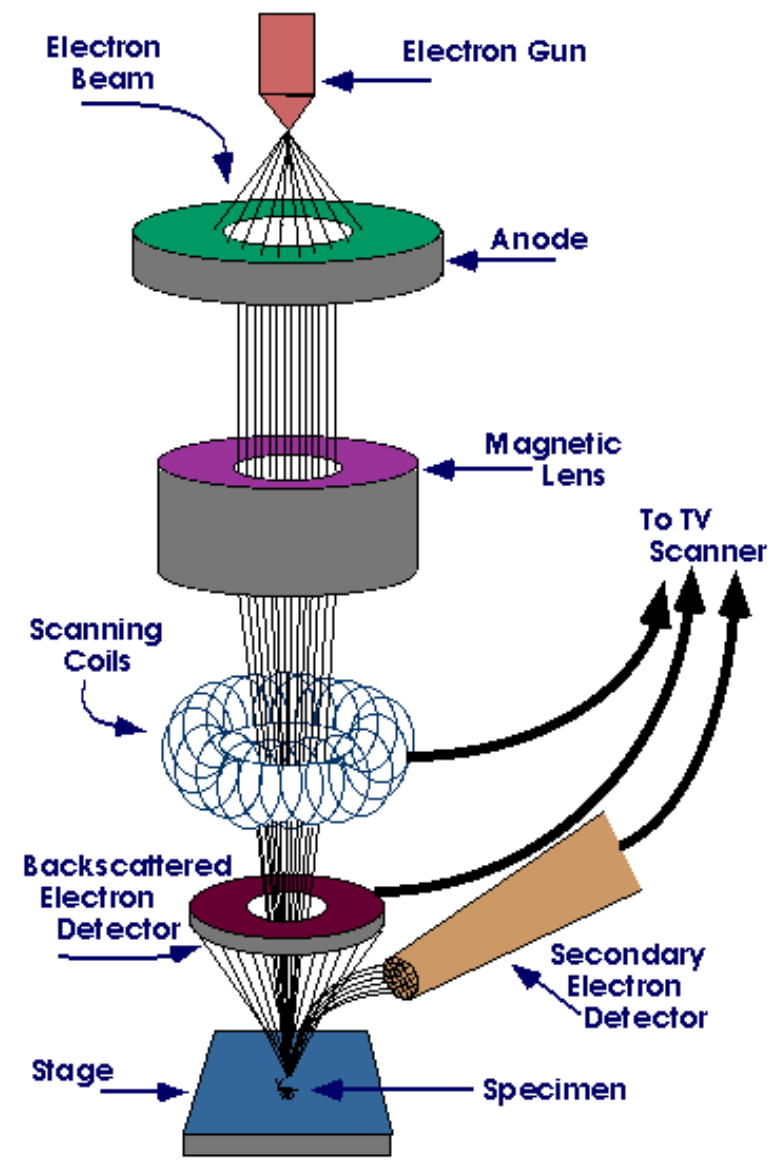

Figure 2.2 The schematic diagram of the Scanning Electron Microscope ${ }^{8}$

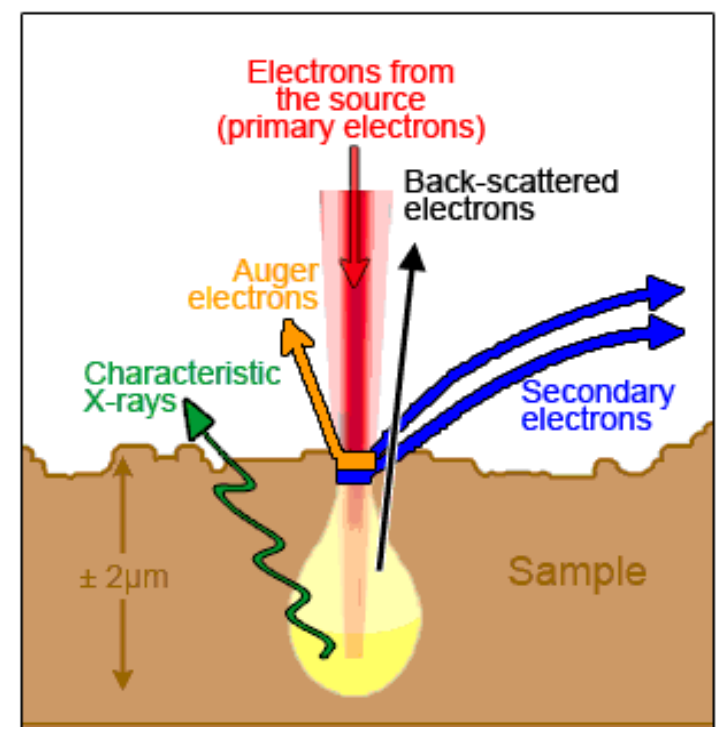

Figure 2.3 Illustration of the sample interaction volume and corresponding particles emitted from sample depth ${ }^{5}$ 
The Secondary Electron (SE) and Backscattered Electron (BSE) detectors are the mostly used detectors. The inelastic scattering of electrons by atoms generate low energy secondary electrons with energy less than the $50 \mathrm{eV} .{ }^{4}$ Due to their low energy, these secondary electrons needed to be accelerated to the photomultiplier via a scintillator. Also, they originate close to the sample surface. The elastic scattering of electrons by atomic nuclei of the sample generate the backscattered electrons with energy greater than the $50 \mathrm{eV}$ up to the energy of the incident beam. Since they have higher energy, they can get scattered out of the interaction volume. The brightness of the backscattered electrons depends on the atomic number of the specimen. Elements with higher atomic numbers are brighter than the elements with lower atomic numbers. ${ }^{8}$ Secondary electrons provide a high resolution image and back scattered electrons provide a relatively low resolution image compared to the secondary electrons. The inelastic scattering near to the surface (Figure 2.3) generate Auger electrons which is useful for surface chemical analysis. ${ }^{4}$ The characteristic $X$ rays are generated at the lower sample depths (Figure 2.3) due to the inelastic scattering of the electrons. ${ }^{4}$

\subsection{Diffuse reflectance spectroscopy}

Perkin Elmer LAMBDA 1050 UV/Vis/NIR Spectrophotometer was used to characterize the powder samples of $\mathrm{Au}-\mathrm{ZnO}$ heterostructures that is mentioned in Chapter 5. There are several reflection types that can be used for material characterization such as specular reflection, diffuse reflection, internal reflection and attenuated total reflection. ${ }^{8}$ The specular reflection usually occurs on smooth polished surfaces. ${ }^{8}$ In fine powder samples, there are a large number of particles with smooth surfaces. Therefore, when the incoming radiation strikes the fine powder sample,

specular reflection occurs on all smooth surfaces (Figure 2.4). ${ }^{10}$ However, since these smooth 
surfaces of the particles are randomly oriented, the incoming radiation reflects in all the directions. Typically, the intensity of the reflected radiation is independent of the viewing angle. ${ }^{8}$

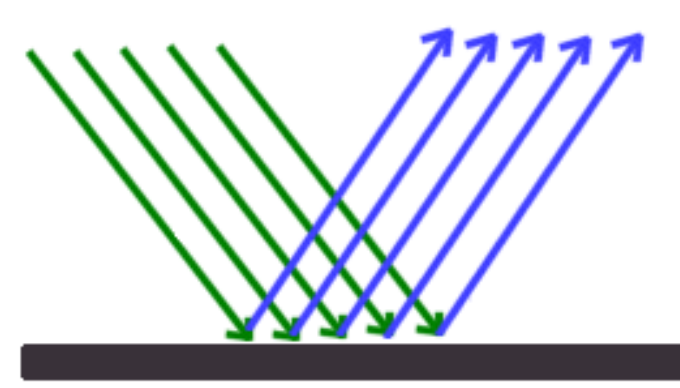

specular reflection

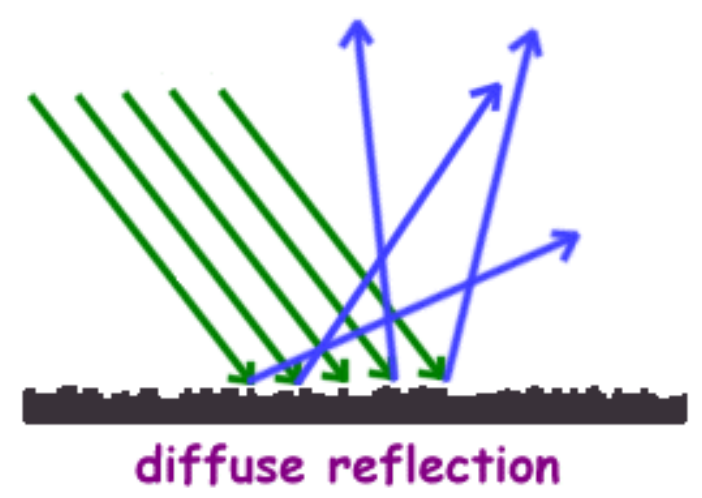

diffuse reflection

Figure 2.4 The schematic diagram showing the difference between specular reflection and diffuse reflection ${ }^{10}$

The most common model for the intensity of reflected radiation that was originally developed by Kubelka-Munk and Fullar-Griffith showed that the relative intensity of the reflected radiation of the powder sample can be given by the following equation. ${ }^{11-13}$

$f\left(R_{\infty}\right)=\frac{\left(1-R_{\infty}\right)^{2}}{2 R_{\infty}}=\frac{k}{S}$

where $R_{\infty}$ is the ratio of reflected intensity of the sample to a non-absorbing standard like finely ground $\mathrm{KCl}, \mathrm{k}$ is the molar absorption coefficient of the analyte and $S$ is the scattering coefficient. Usually, the percentage of $R_{\infty}$ is plotted with the wavelength. ${ }^{8}$ 


\subsection{UV-VIS absorption spectroscopy}

Agilent $8453 \mathrm{UV} / \mathrm{Vis} / \mathrm{NIR}$ Spectrophotometer was used with a quartz cuvette to obtain the absorption spectra of $\mathrm{Au}-\mathrm{ZnO}$ nanopyramids dissolved in hexane. Also, it was used to obtain absorption spectra of methylene blue dye during the dye degradation experiments discussed in Chapter 6. Ultra Violet (190 nm to $400 \mathrm{~nm}$ ) - Visible (400 nm to $800 \mathrm{~nm}$ ) absorption spectroscopy is commonly used for the quantitative analysis of a variety of inorganic, organic and biological species. ${ }^{8,14}$ Incoming radiation is absorbed by the atoms, ions or molecules in an analyte solution due to the electron transition from lower energy states to the higher energy states. According to the Beer-Lambert law, absorption is linearly proportional to the concentration and path length of the sample as follows: ${ }^{13,14}$

$A=-\log T=\log \frac{P_{0}}{P}=\epsilon b c$

where $A$ is the absorbance, $T$ is the transmittance, $P_{0}$ is incident the radiation power, $P$ is the transmitted radiation power, $\epsilon$ is the molar absorptiviy, $b$ is the path length of the sample and $c$ is the concentration of the absorber. ${ }^{8}$

As shown in Figure 2.5, two air-wall interfaces and two solution-wall interfaces are in the beam path and those interfaces are responsible for beam attenuation. ${ }^{8,15}$ Also, the scattering from larger atoms, ions or molecules and absorption from the walls of the glass cell affects the transmitted radiation power. ${ }^{8}$ A glass cell with only the solvent is usually used as a reference to remove these affects. 


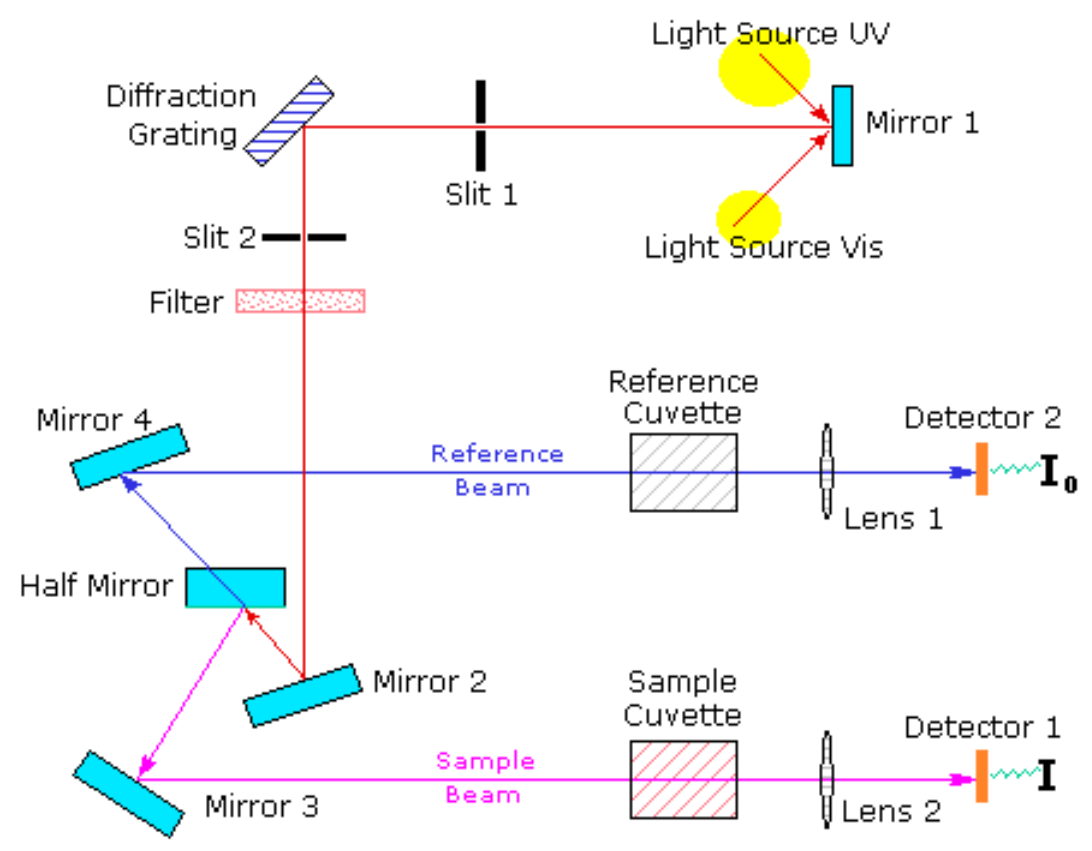

Figure 2.5 The schematic diagram of the UV-Vis spectrophotometer ${ }^{15}$

\subsection{X-ray diffractometry}

PANalytical X'pert Pro powder X-Ray diffractrometer was used to take the X-ray diffraction data in order to identify the crystal structures of $\mathrm{Au}-\mathrm{ZnO}$ heterostructures as well as for Rietveld refinement analysis. X-ray diffraction is a very common experimental technique that is used to investigate the structure of crystalline materials. X-ray beams are generated in four different ways such as by bombarding a metal target with a beam of high energy electrons (Figure 2.6), by exposing a substance to a primary beam of X-rays to generate a secondary beam of X-ray fluorescence, by a radioactive decay process and from synchrotron radiation sources. ${ }^{8}{ }^{8} 16$ When the $\mathrm{X}$-ray beam reaches the atomic planes of the material; transmittance, absorption, refraction, scattering and diffraction can be occurred. By collecting the diffracted X-ray photons, it is possible to plot a graph of intensity versus scattering angle. Each crystalline element has a unique X-ray 
diffraction pattern which is characteristic of the crystalline structure of that element. There are two main requirements for X-ray diffraction.

1. The spacing between the layers of atoms is roughly equal to the wavelength of the X-ray radiation.

2. The scattering centers must be spatially distributed in a highly regular way Using the Bragg's law (41), it is possible to calculate the distance between two atomic planes ( $\mathrm{d}$ spacing) in a crystalline structure. Bragg's law:

$n \lambda=2 d \sin \theta$

where $n$ is the order of the diffracted beam, $\lambda$ is the wavelength of the $\mathrm{X}$-ray radiation, $\theta$ is the incidence angle of the $\mathrm{X}$-ray beam and $d$ is the distance between the adjacent atomic planes.

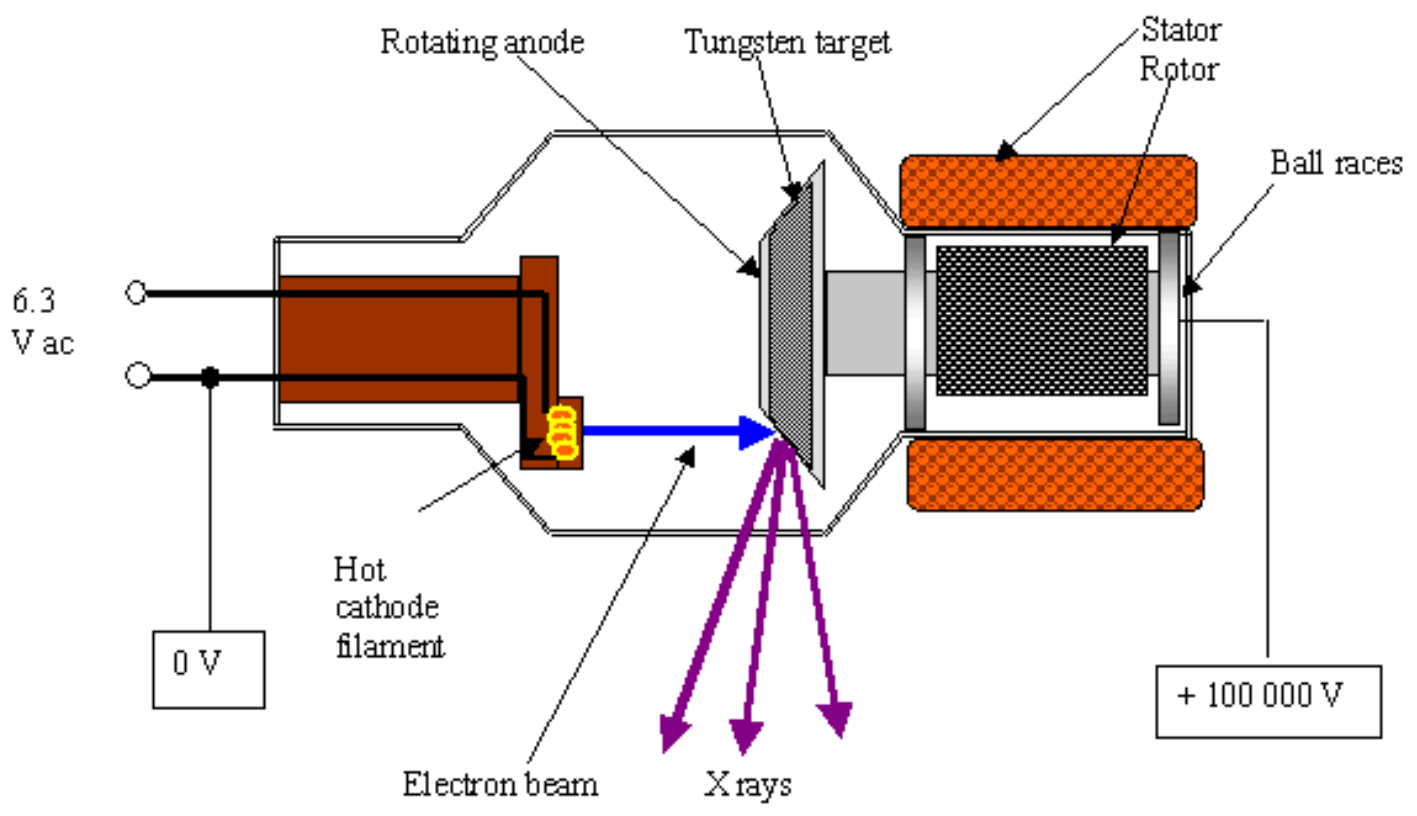

Figure 2.6 The schematic diagram of the X-ray generation from high energy electrons ${ }^{16}$ 


\subsection{Fourier Transform Infra-Red spectroscopy (FTIR)}

Perkin Elmer - Spectrum 100 Optica FTIR spectrometer was used to obtain FTIR spectra of Au$\mathrm{ZnO}$ nanopyramids before and after $\mathrm{O}_{2}$ plasma etching in order to confirm the removal of organic ligands. Since molecules are made with a unique combination of atoms, their vibrational spectra are also unique. ${ }^{17}$ Also, these vibrations are in IR region which means IR spectra can be used to identify the materials. Since the size of the IR peak is proportional to the amount of the material present in the sample; this technique was used to confirm the removal of the organic molecules. In FTIR spectrometers, an interferometer is used to measure all the frequencies simultaneously and it generates a unique signal (interferogram) which contains the information about all the IR frequencies generated from the source. ${ }^{17}$ This signal is converted to a frequency spectrum by a computer algorithm and this method is extremely fast compared to the old dispersive type instruments. Figure 2.7 shows the schematic diagram of the generation of an FTIR spectrum. ${ }^{18}$

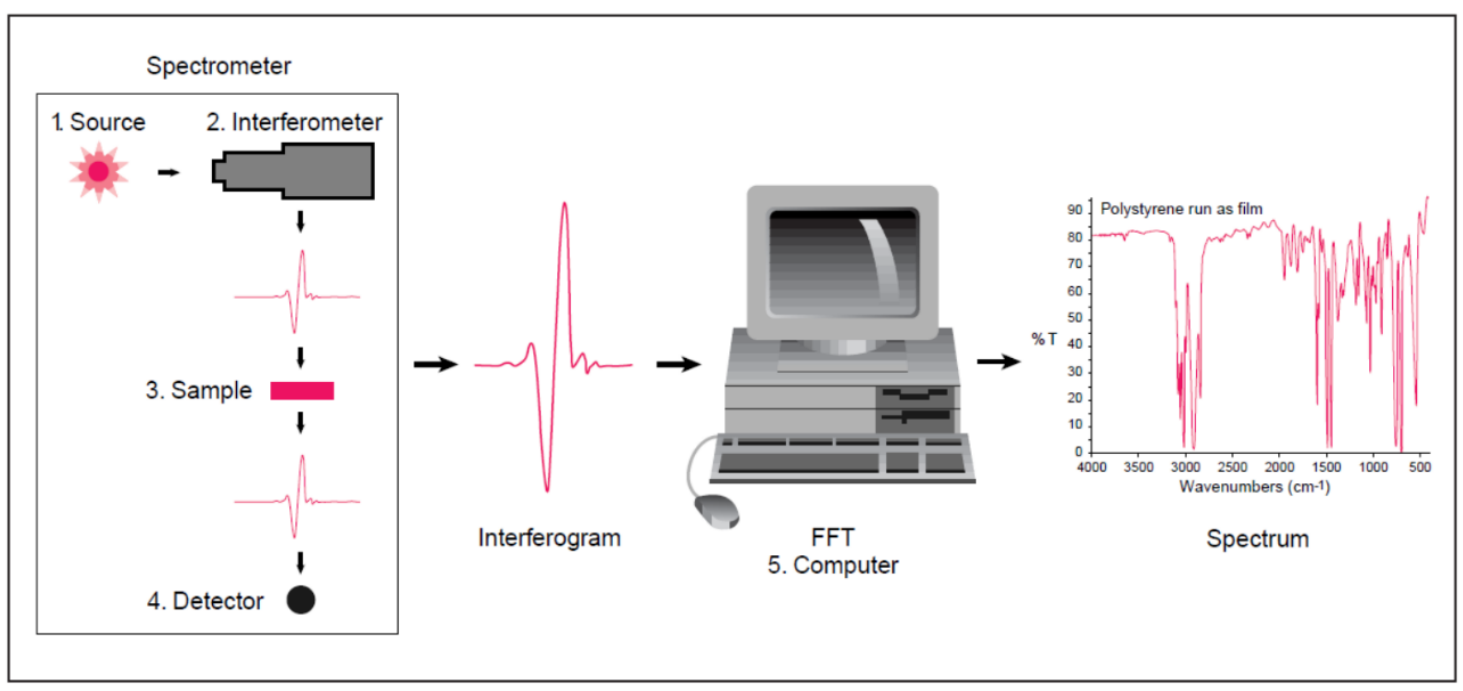

Figure 2.7 The schematic diagram of the generation of FTIR spectrum ${ }^{18}$ 


\subsection{Gas Chromatography (GC)}

Perkin Elmer Clarus 600 Gas Chromatograph equipped with Thermal Conductivity Detector (TCD) and Flame Ionization Detector (FID) was used to identify the $\mathrm{CO}_{2}$ reduction products that are discussed in Chapter 5. The basic principle of GC is volatilization of the sample in a heated inlet followed by the separation of components in the special columns and detect at the detector according to the retention time of each component. ${ }^{19}$ Therefore, materials which vaporize without decomposition can be analyzed using the GC. In general, a carrier gas or mobile phase (usually $\mathrm{H}_{2}$ or He) transfers the sample from the inlet to the detector. The stationary phase is coated in the inner wall of the column. The separation of the components depend on the distribution of each component between the mobile phase and the stationary phase. ${ }^{19}$ For an example, a component which stays a little longer in the stationary phase will leave the column later. In other words, the peak corresponding to the component that stays a longer time in the stationary phase can be seen at end of the "intensity versus retention time" graph. There are several detectors specifically designed for the GC such as TCD (Thermal Conductivity Detector) and FID (Flame Ionization Detector).

\subsubsection{Thermal Conductivity Detector (TCD)}

Thermal Conductivity Detector (TCD) is a non-destructive detector and it consists of two channels (Figure 2.8): one for the mobile phase with the eluate and the other for the pure mobile phase. ${ }^{20}$ Since TCD is non-destructive, it is possible to transfer the eluate to a different detector after the TCD. The TCD acts as a Wheatstone bridge circuit with four filaments (resistors). ${ }^{19}$ The components with different thermal conductivities change the temperature and resistance of the 
filament compared to the reference channel. Since helium has a higher thermal conductivity than the most analytes, usually helium is used as the mobile phase.

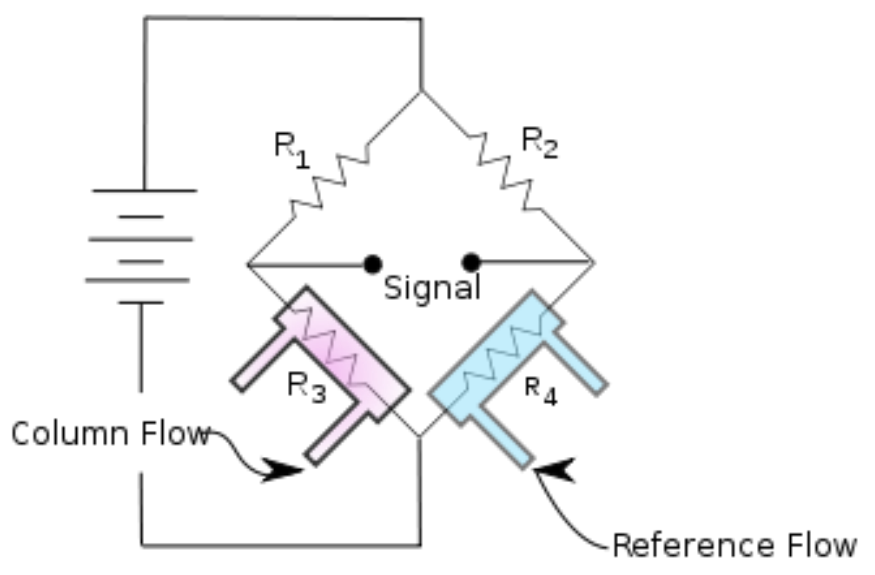

Figure 2.8 The schematic diagram of the thermal conductivity detector ${ }^{20}$

\subsubsection{Flame Ionization Detector (FID)}

FID is mostly used to detect the components which have $\mathrm{C}-\mathrm{H}$ bonds. ${ }^{19}$ FID needs hydrogen gas and air to make a flame at the tip of the flame jet (Figure 2.9). ${ }^{21}$ Analyte eluate burns at the flame and produces ions and the amount of the ions is proportional to the concentration of the component. The positive ions are collected by the negatively biased collector and are amplified electronically. ${ }^{21}$

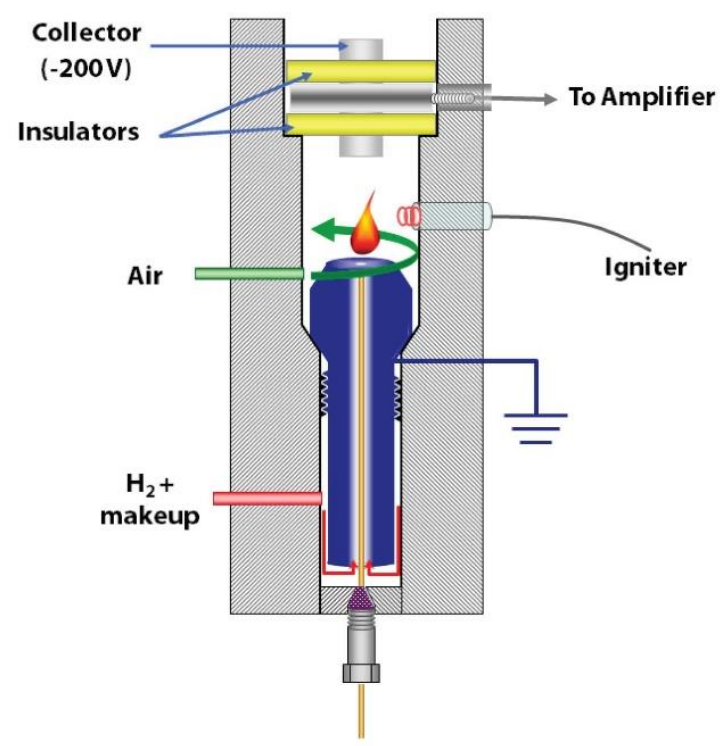

Figure 2.9 The schematic diagram of the flame ionization detector ${ }^{21}$ 


\subsection{Oxygen $\left(\mathrm{O}_{2}\right)$ plasma etching}

March PX-250 $\mathrm{O}_{2}$ Plasma Asher was used to remove the excess organic ligands in Au- $\mathrm{ZnO}$ nanopyramids after the wet chemical synthesis. Plasma etching is a chemical etching method which means the unnecessary molecules can be removed from the sample after a chemical reaction between the plasma and the atoms in the sample. In general, $\mathrm{O}_{2}$ plasma is generated in a vacuum chamber which has a constant $\mathrm{O}_{2}$ gas flow and a radio frequency potential generator. ${ }^{22} \mathrm{O}_{2}$ gas is partially ionized when it is exposed to the radio frequency potential at the reduced pressure $(\sim 300$ mTorr in this work). As a result of this ionization, it creates atomic oxygen which are more active than the oxygen molecules. ${ }^{6}$ Atomic oxygen reacts with organic molecules and converts them in to water and carbon dioxide that will be removed from the chamber via a vacuum pump (Figure 2.10). ${ }^{23}$

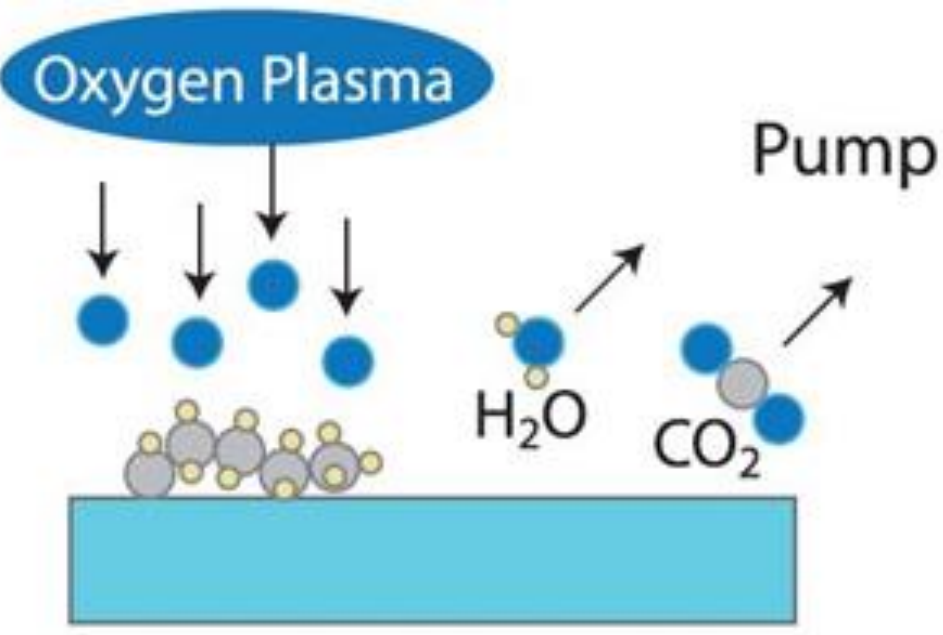

Figure 2.10 The schematic diagram of the $\mathrm{O}_{2}$ plasma etching ${ }^{23}$ 


\subsection{Raman spectroscopy}

Renishaw InVia Raman Spectrometer with a Crystal 532-100 green laser was used to investigate the plasmonic heating effects of $\mathrm{Au}-\mathrm{ZnO}$ heterostructures. In general, Raman spectroscopy is used to identify the vibrational states of the materials based on the inelastic scattering of monochromatic radiation. ${ }^{10,24}$ The changing polarizability of the molecule is responsible for the Raman scattering. ${ }^{25}$ The scattered light can be divided in to two components as Rayleigh scattering which has the same frequency $\left(v_{\mathrm{ex}}\right)$ as incident light (elastic scattering) and Raman scattering which has a slightly higher or lower frequency $\left(v_{\mathrm{ex}} \pm v_{\mathrm{v}}\right)$ compared to the incident light. $v_{\mathrm{v}}$ is the vibrational frequency of the molecule. ${ }^{22}$ Also, Raman scattering is very weak and usually the signal intensity is $10^{5}$ times lower than the incident light intensity. ${ }^{22}$ The higher frequencies $\left(v_{\mathrm{ex}}+v_{\mathrm{v}}\right)$ and lower frequencies $\left(v_{\mathrm{ex}}-v_{\mathrm{v}}\right)$ are called as anti-stokes peaks and stokes peaks respectively (Figure 2.11). ${ }^{8}$,

${ }^{22}$ Under the laser illumination, molecules get excited in to a virtual energy state from a low energy vibrational state and then relax back. Since the population is higher in ground vibrational state compared first excited vibrational state, stokes peaks (Figure 2.11) are relatively more intense than the anti-stokes peaks.

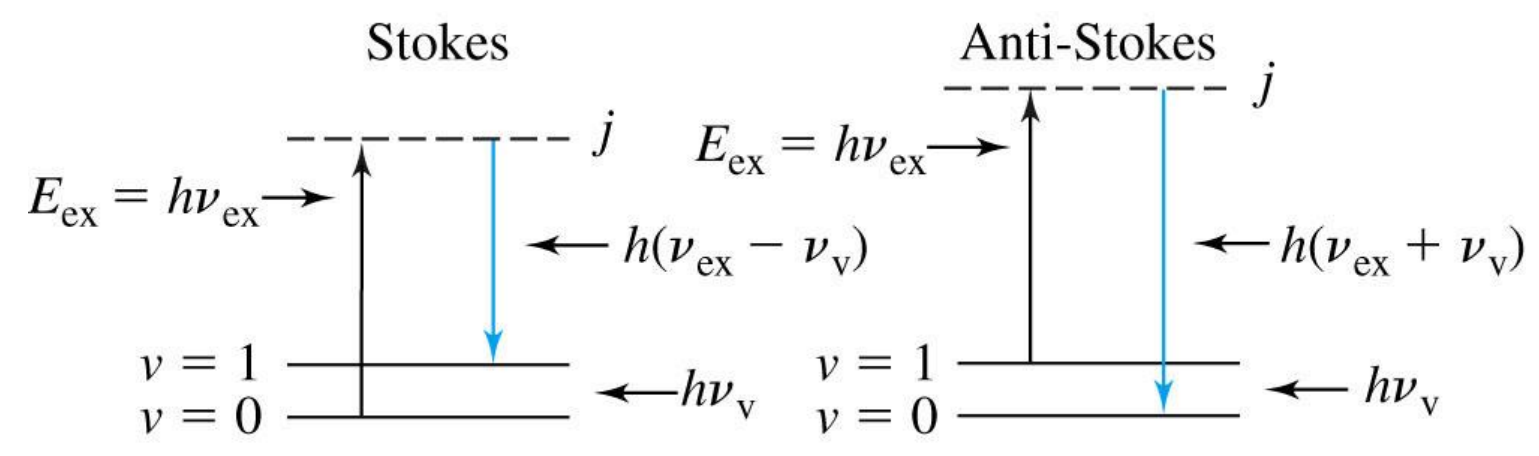

Figure 2.11 The schematic diagram of the stoke and anti-stokes lines ${ }^{8}$ 


\section{References}

1. PRINCIPLES OF THE TRANSMISSION ELECTRON MICROSCOPE (TEM) http://em-outreach.ucsd.edu/web-course/Sec-I.A/Sec-I.A.html\#Origin, Accessed 04/13/2015, 2015.

2. The Electron Gun, http://www.snaggledworks.com/em_for_dummies/gun.html, Accessed 04/13/20115, 2015.

3. A. Marshall and A. L. Koh, Transmission Electron Microscope (TEM) Facility, http://web.stanford.edu/group/snl/tem.htm, Accessed 04/13/2015, 2015.

4. B. D. Fahlman, Materials Chemistry, Springer Netherlands, 2011.

5. How does an EM work?, http://www.vcbio.science.ru.nl/en/fesem/info/principe/, Accessed 04/14/2015, 2015.

6. Y. Ding, Fundamental Theory of Transmission Electronic Microscopy, http://www.nanoscience.gatech.edu/zlwang/research/tem.html, Accessed 04/13/2015, 2015.

7. Limits to Resolution in the Transmission Electron Microscope, http://www.ou.edu/research/electron/bmz5364/resolutn.html, Accessed 04/13/2015, 2015.

8. D. A. Skoog, F. J. Holler and S. R. Crouch, Instrument Analysis, Cengage Learning India Private Limited, 2009.

9. SEM Introduction: an Overview of Scanning Electron Microscopy, http://www.understanding-cement.com/sem-introduction.html, Accessed 04/14/2015, 2015. 
10. Behavior of Light as a Wave, http://www.ducksters.com/science/physics/light_as_a_wave.php, Accessed 04/14/2015, 2015.

11. F. P. Kubelka and Z. Munk, Tech Phys, 1931, 12.

12. P. Kubelka, J. Opt. Soc. Am., 1948, 38, 448-448.

13. M. P. Fuller and P. R. Griffiths, Analytical Chemistry, 1978, 50, 1906-1910.

14. S. Petrozzi, Practical Instrumental Analysis: Methods, Quality Assurance and Laboratory Management, 2012.

15. UV-Visible Spectroscopy, https://www2.chemistry.msu.edu/faculty/reusch/virttxtjml/Spectrpy/UV-Vis/uvspec.htm, $04 / 14 / 2015$

16. Medical Physics Tutorial 7 - X-Rays, http://www.antonineeducation.com/Pages/Physics_5_Options/Medical_Physics/MED_07/med_phys_7.htm, Accessed 04/14/2015, 2015.

17. Introduction to Raman Spectroscopy, http://bwtek.com/raman-introduction-to-ramanspectroscopyl, Accessed 04/15/2015, 2015.

18. Introduction to FTIR http://mmrc.caltech.edu/FTIR/FTIRintro.pdf, Accessed 04/14/2015, 2015.

19. O. D. Sparkman, Z. E. Penton and F. G. Kitson, Gas Chromatography and Mass Spectrometry, 2011.

20. Thermal conductivity detector, https://en.wikipedia.org/wiki/Thermal_conductivity_detector, Accessed 04/28/2015, 2015. 
21. M. Klee, GC Solutions \#11: The Flame Ionization Detector, http://www.sepscience.com/Techniques/GC/Articles/208-/GC-Solutions-11-The-FlameIonization-Detector, Accessed 04/14/2015, 2015.

22. J. R. Ferraro, K. Nakamoto and C. W. Brown, in Introductory Raman Spectroscopy (Second Edition), ed. J. R. F. N. W. Brown, Academic Press, San Diego, 2003, pp. 1-94.

23. Cold Gas Plasma Surface Modification - Optimize Plastics Bonding Adhesion, http://www.adhesionbonding.com/2012/04/30/cold-gas-plasma-surface-modificationoptimize-plastics-bonding-adhesion/, Accessed 04/15/2015, 2015.

24. J. Javier, An Introduction to Raman Spectroscopy: Introduction and Basic Principles, http://www.spectroscopynow.com/details/education/sepspec1882education/AnIntroduction-to-Raman-Spectroscopy-Introduction-and-BasicPrinciples.html?\&tzcheck=1, Accessed 04/15/2015, 2015.

25. H. Kuzmany, Solid-State Spectroscopy, Springer. 


\section{Chapter 3}

\section{Vibrational modes and phonon spectral density of Au20 clusters}

\subsection{Introduction}

Vibrational spectroscopy can be used as an efficient tool to identify the different isomers of small gold clusters. ${ }^{1}$ Using vibrational spectroscopy, Gruene et al confirmed the pyramidal structure (tetrahedral) for neutral $\mathrm{Au}_{20}{ }^{2}$ The tetrahedral $\mathrm{Au}_{20}$ cluster was widely studied due its high symmetry. The group theory predicted 23 non-degenerate vibrational modes (Equation 3.1) with irreducible representation of tetrahedral $\mathrm{Au}_{20} .^{3}$

$\Gamma_{\mathrm{vib}}=4 \mathrm{~A}_{1}+\mathrm{A}_{2}+5 \mathrm{E}+5 \mathrm{~T}_{1}+8 \mathrm{~T}_{2}$

(Equation 3.1)

According to the group theory, 9 vibrational modes with symmetry of $A_{1}$ and $E$ are Raman active and 8 modes with $T_{2}$ symmetry are both Raman and IR active. The modes with $A_{2}$ and $T_{1}$ are silent for both Raman and IR. ${ }^{3}$ In previous work, vibrational modes of $\mathrm{Au}_{20}$ that were calculated using different approximations, methods and basis can be found. ${ }^{1-7}$

Geometrical structure optimization, molecular dynamic (MD) simulations and vibrational modes calculations were performed using the FIREBALL ${ }^{8-10}$; a local-orbital density functional theory (DFT) package. For all the calculations BLYP method and SNP basis were used. The phonon density of states of tetrahedral $\mathrm{Au}_{20}$ were calculated by taking the Fourier transform of the velocities from MD simulations. 


\subsection{Geometrical structure optimization of $\mathrm{Au}_{20}$}

The geometrical structure optimization was run until it reached a very stable structure. The time step size was $1 \mathrm{fs}$ and RMS cutoff was set as 0.05 . The structure reached RMS value after 823 time steps and the calculated band gap was $2.18 \mathrm{eV}$. Figure 3.1 shows the optimized structure of $\mathrm{Au}_{20}$ and Figure 3.2 shows the variation of the total energy during the geometrical structure optimization. Tetrahedral $\mathrm{Au}_{20}$ cluster consists of 3 types of atoms such as 4 apex atoms with coordination number $=3,4$ center atoms with coordination number $=9$ and 12 edge atoms with coordination number $=6 \cdot^{3-6}$ Also, it contains $54 \mathrm{Au}$-Au bonds including 6 bonds between middle edge atoms, 24 bonds between center atoms and edge atoms, 12 bonds between middle edge atoms in opposite edges and 12 bonds between apex atoms and edge atoms. ${ }^{7}$

The bond distances and bond angles of optimized $\mathrm{Au}_{20}$ structure were measured using the $\mathrm{Jmol}$; an open-source Java viewer for chemical structures in 3D. ${ }^{11}$ Table 3.1 shows the detail comparison of the main parameters of the $\mathrm{Au}_{20}$ cluster with previous work. Differences between calculated values from previous literature can be seen in Table 3.1 since the calculated values largely depend on the calculation method and basis set. In this work, the band gap for the tetrahedral $\mathrm{Au}_{20}$ cluster was found to be $2.18 \mathrm{eV}$ and this value is fairly large when compared to the experimental value of $1.77 \mathrm{eV}$ measured by Li et al ${ }^{6}$ using X-ray photoelectron spectroscopy. Also, Flores ${ }^{7}$ calculated the band gap as $1.90 \mathrm{eV}$ using PBE method and DNP basis. Molina et $\mathrm{al}^{3}$ calculated the band gap of $\mathrm{Au}_{20}$ cluster as $1.92 \mathrm{eV}$ and $2.96 \mathrm{eV}$ using BP86 and B3LYP methods respectively. However Molina et $\mathrm{al}^{3}$ used the same LANL2DZ basis for both calculations, which means almost $\sim 1 \mathrm{eV}$ difference of the band gap can be observed just for the difference in the calculation method. 
Our calculated a-e bond distances (see the Figure 3.1) well matched the values calculated by Kryachko et al ${ }^{5}$ and Flores. ${ }^{7}$ Also, the longest e-e bond distance in this work was compatible with the values of Kryachko et $a l^{5}$ while the c-e bond distances agreed with the values published by Kryachko et al $^{5}$ and Flores. ${ }^{7}$ The c-c bond distance was within the error less than $10 \%$ of the value calculated by the Molina et al. ${ }^{3}$ The Au-Au-Au bond angles which were calculated in this work well matched the values reported by De et $a l^{4}$ within the error less than $5 \%$. Also, to the date there is no theoretical calculations of $\mathrm{Au}_{20}$ cluster available with BLYP method and SNP basis.

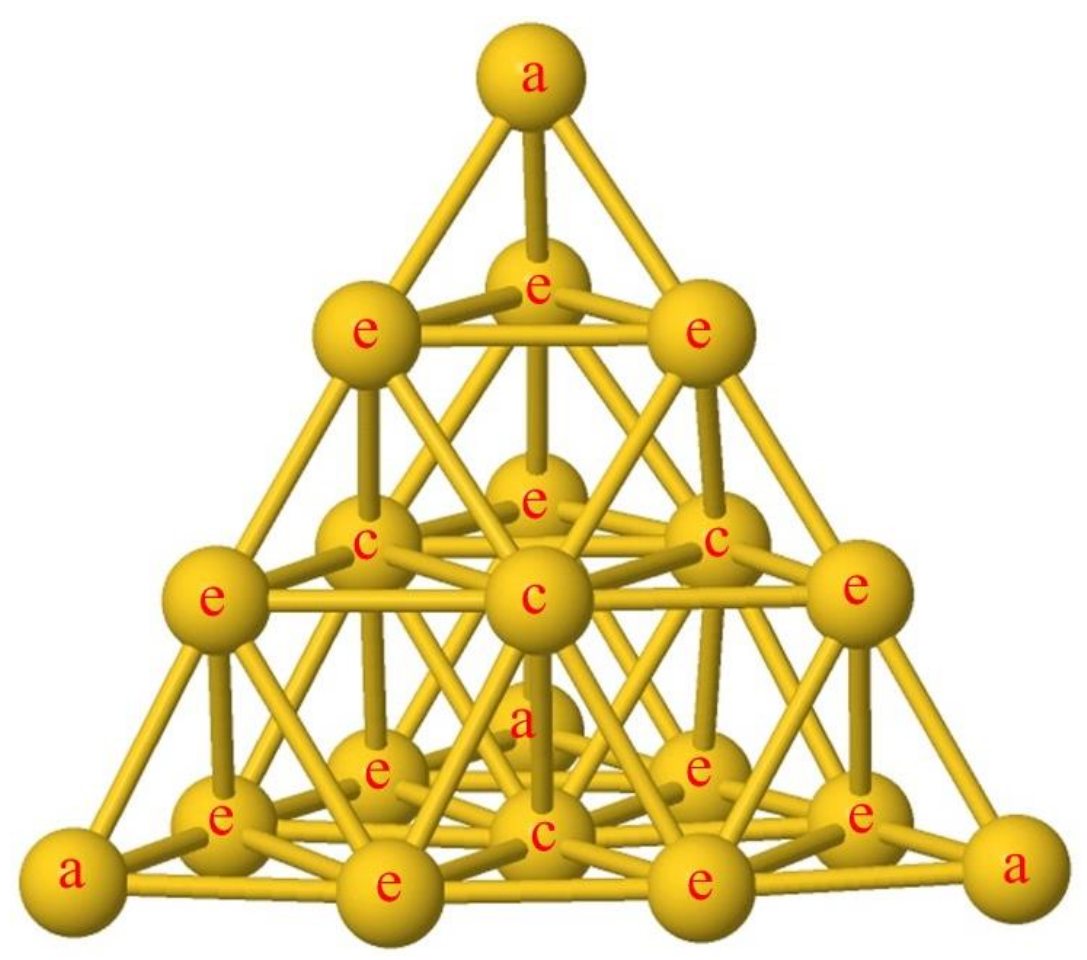

Figure 3.1 Optimized tetrahedral structure of $\mathrm{Au}_{20}$, 'a','c' and 'e' represent apex, center and edge atoms 


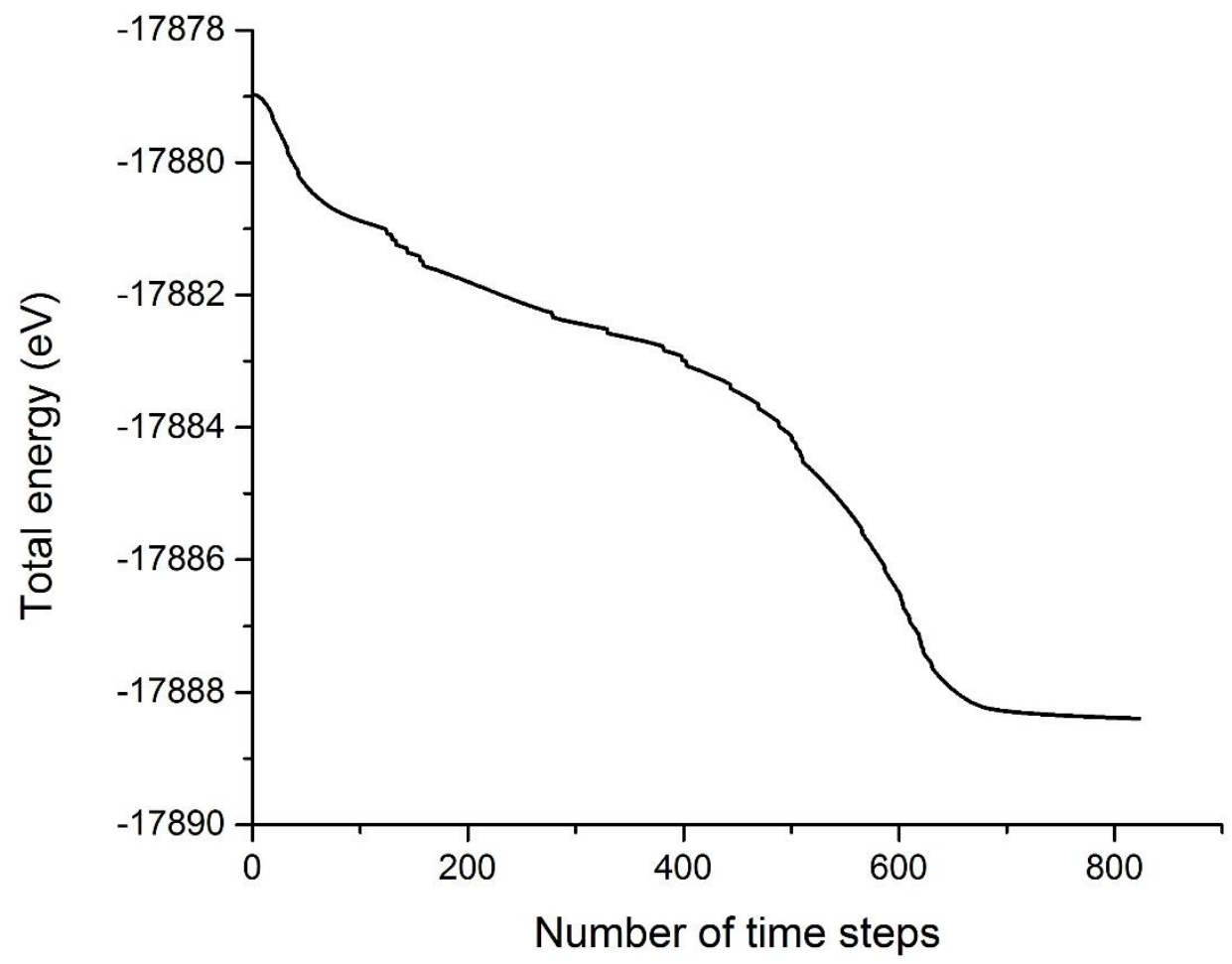

Figure 3.2 Variation of the total energy during the geometrical structure optimization

\subsection{Vibrational modes of $\mathrm{Au}_{20}$}

The vibrational modes of $\mathrm{Au}_{20}$ cluster were calculated using the eigen values of dynamical matrix which was calculated using the FIREBALL package. The dynamical matrix consists of second derivatives of the energy with respect to the geometry of elements. ${ }^{12}$ Forces are the first derivatives with respect to the geometry. The second derivatives were calculated using the two forces in to opposite directions and the displacement. As the first step, one atom of the optimized structure was moved in to one direction and self-consistent field ${ }^{13}$ (SCF) calculations were performed to obtain the force. Then, the same atom was moved in to the opposite direction and performed SCF calculations and obtained the force. After that, the difference of these two forces was divided by the displacement of the atom in order to get the second derivatives of the energy with respect to the geometry. This procedure was performed for all $\mathrm{Au}$ atoms in $\mathrm{x}, \mathrm{y}$ and $\mathrm{z}$ directions. 
Table 3.1 Comparison of main parameters of the tetrahedral $\mathrm{Au}_{20}$ neutral cluster after the geometrical structure optimization.

\begin{tabular}{|c|c|c|c|c|c|c|c|c|}
\hline Reference & Method & Basis & $\begin{array}{c}\Delta \mathbf{E}_{\mathrm{HL}} \\
(\mathrm{eV})\end{array}$ & $\begin{array}{l}\text { HOMO } \\
(\mathrm{eV})\end{array}$ & $\begin{array}{c}\text { Highest } \\
\text { vibrational } \\
\text { frequency } \\
\left(\mathbf{c m}^{-1}\right)\end{array}$ & $\begin{array}{c}\text { Lowest } \\
\text { vibrational } \\
\text { frequency } \\
\quad\left(\mathrm{cm}^{-1}\right)\end{array}$ & $\begin{array}{c}\text { Au-Au distances } \\
\text { (§) }\end{array}$ & $\begin{array}{c}\text { Au-Au-Au bond angles } \\
(\AA)\end{array}$ \\
\hline This work & BLYP & SNP & 2.18 & -4.64 & 195.80 & 25.32 & $\begin{array}{l}\text { a-e (longest) }=2.76 \\
\text { a-e (shortest) }=2.75 \\
\text { e-e (longest) }=3.09 \\
\text { e-e (shortest) }=2.68 \\
\text { c-e (longest) }=2.89 \\
\text { c-e (shortest) }=2.81 \\
\text { c-c (longest) }=3.44 \\
\text { c-c (shortest) }=3.06\end{array}$ & $\begin{array}{l}\text { e-a-e (largest) }=68.3 \\
\text { e-a-e (smallest) }=63.8 \\
\text { e-c-e (largest) }=177.2 \\
\text { e-c-e (smallest) }=163.4 \\
\text { a-e-e (largest) }=178.2 \\
\text { a-e-e (smallest) }=175.5 \\
\text { e-e-e (largest) }=63.1 \\
\text { e-e-e (smallest) }=57.2\end{array}$ \\
\hline $\begin{array}{l}\text { Sekhar De } \\
\text { et } \mathrm{al}^{4}\end{array}$ & B88 & RECP & & & & & $\begin{array}{l}\mathrm{a}-\mathrm{e}=2.80 \\
\mathrm{e}-\mathrm{e}=2.74 \\
\mathrm{c}-\mathrm{e}=2.93 \\
\mathrm{c}-\mathrm{c}=3.34\end{array}$ & $\begin{array}{l}\mathrm{e}-\mathrm{a}-\mathrm{e}=67.0 \\
\mathrm{e}-\mathrm{c}-\mathrm{e}=170.4 \\
\mathrm{a}-\mathrm{e}-\mathrm{e}=175.6 \\
\mathrm{e}-\mathrm{e}-\mathrm{e}=60.0\end{array}$ \\
\hline $\begin{array}{l}\text { Kryachko et } \\
\mathrm{al}^{5}\end{array}$ & BP86 & LANL2DZ & & -6.02 & 172 & 28 & $\begin{array}{l}\text { a-e }=2.76 \\
\text { e-e (longest) }=3.01 \\
\text { e-e (shortest) }=2.71 \\
\mathrm{c}-\mathrm{e}=2.86\end{array}$ & \\
\hline $\begin{array}{l}\text { Kryachko et } \\
\mathrm{al}^{5}\end{array}$ & B3LYP & LANL2DZ & & -6.40 & 161 & 26 & $\begin{array}{l}\mathrm{a}-\mathrm{e}=2.80 \\
\mathrm{e}-\mathrm{e} \text { (longest) }=3.08 \\
\mathrm{e}-\mathrm{e} \text { (shortest) }=2.73 \\
\mathrm{c}-\mathrm{e}=2.91\end{array}$ & \\
\hline Flores $^{7}$ & PBE & DNP & 1.9 & & 181.4 & 16.2 & $\begin{array}{l}\mathrm{a}-\mathrm{e}=2.75 \\
\mathrm{e}-\mathrm{e}(\text { longest })=3.00 \\
\mathrm{e}-\mathrm{e}(\text { shortest })=2.71 \\
\mathrm{c}-\mathrm{e}=2.86\end{array}$ & \\
\hline $\begin{array}{l}\text { Molina et } \\
\mathrm{al}^{3}\end{array}$ & BP86 & LANL2DZ & 1.92 & -6.03 & 172.35 & 29.13 & $\begin{array}{l}2.71,2.76,2.87,3.01, \\
3.13\end{array}$ & \\
\hline $\begin{array}{l}\text { Molina et } \\
\mathrm{al}^{3}\end{array}$ & B3LYP & LANL2DZ & 2.96 & -6.41 & 162.22 & 26.42 & $\begin{array}{l}2.73,2.79,2.91,3.08, \\
3.21\end{array}$ & \\
\hline Li et $\mathrm{al}^{6}$ & $\begin{array}{l}\text { X-Ray } \\
\text { Photoele } \\
\text { ctron } \\
\text { Spectros } \\
\text { copy } \\
\text { (experi } \\
\text { mental) }\end{array}$ & & 1.77 & & & & $\begin{array}{l}2.68,2.71,2.83,2.97 \\
3.12 \text { (calculated) }\end{array}$ & \\
\hline
\end{tabular}


60 frequencies were obtained since there are $3 n\left(n=\right.$ number of atoms) degrees of freedom in $\mathrm{Au}_{20}$ cluster in $\mathrm{x}, \mathrm{y}$ and $\mathrm{z}$ directions. However, $3 \mathrm{n}$ degrees of freedom include translational and rotational motion of the $\mathrm{Au}_{20}$ cluster as well. The first six frequencies of the FIREBALL output correspond to the 3 rotational frequencies and 3 translational frequencies. The remaining 54 frequencies are actual vibrations. The highest vibrational frequency found to be as $195.80 \mathrm{~cm}^{-1}$ in this calculations which is $8 \%$ higher than the calculated value by Flores. ${ }^{7}$ The lowest vibrational frequency found to be as $25.32 \mathrm{~cm}^{-1}$, which well agrees with the calculations done by the Kryachko et al. ${ }^{5}$ All 54 vibrational modes can be seen in Figure 3.3. Due to the tetrahedral structure of $A_{20}$, there is a large number of degenerate modes. The 23 distinct vibrational modes (Equation 3.1) were identified by Molina et al. ${ }^{3}$

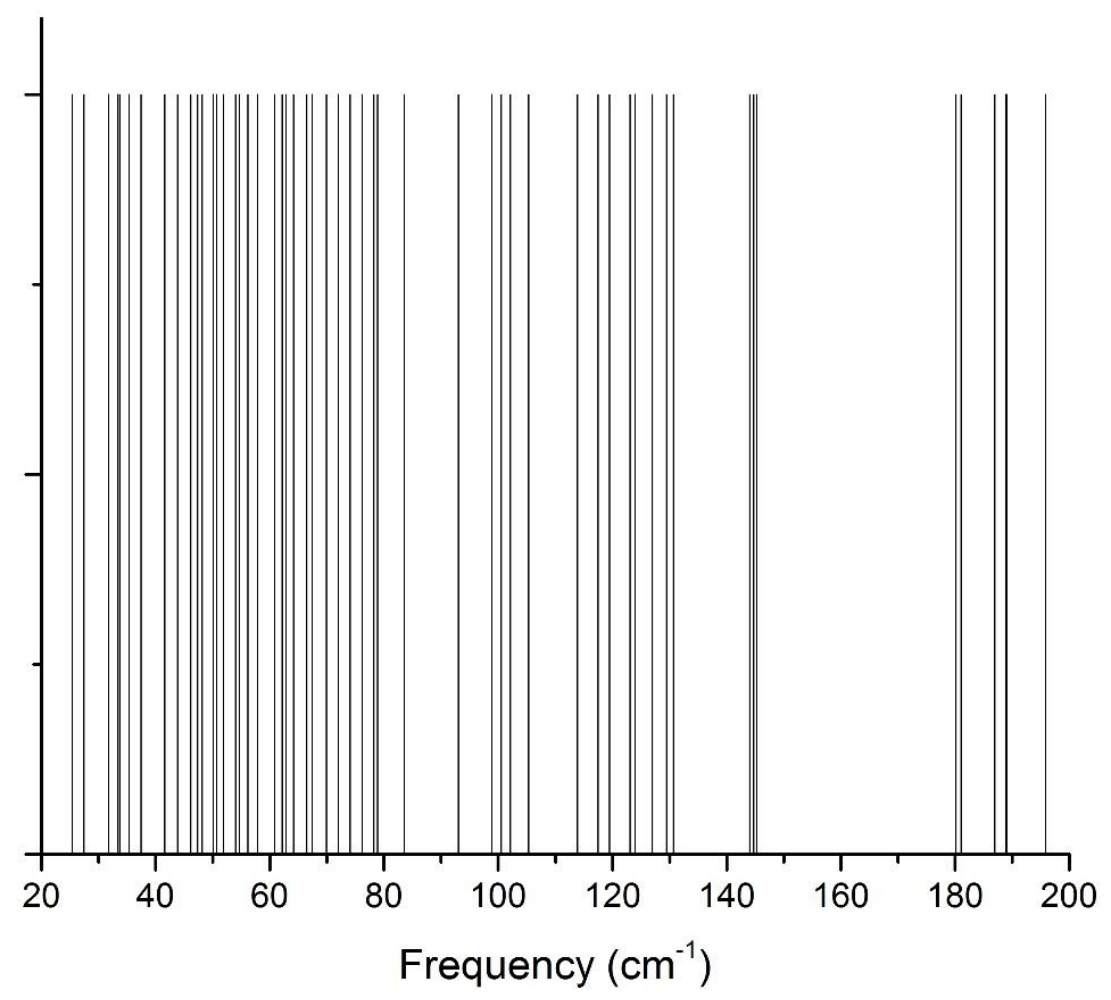

Figure 3.3 Vibrational modes of $\mathrm{Au}_{20}$ cluster 
The schematic diagrams of eigen vectors from Molina et $a l^{3}$ were used to identify the symmetry of vibrational modes of this work. Figure 3.4 shows the eigen vectors for highest vibrational mode $\left(195.80 \mathrm{~cm}^{-1}\right)$ which is the $4^{\text {th }}$ mode of $A_{1}$ symmetry. Only the tangential atoms were involved in this $A_{1}$ vibrational mode and there is no considerable displacement in four center atoms. The Raman breathing mode ( $3^{\text {rd }}$ mode of $A_{1}$ symmetry) with the frequency of $105.32 \mathrm{~cm}^{-1}$ can be seen in Figure 3.5. This breathing mode shows identical radial displacement in all 20 atoms. Also, degeneracy of some symmetries were identified. For an example, the $6^{\text {th }}$ mode of $T_{2}$ symmetry has a degeneracy of 3 around $129.05 \mathrm{~cm}^{-1}$ (Figure 3.6)

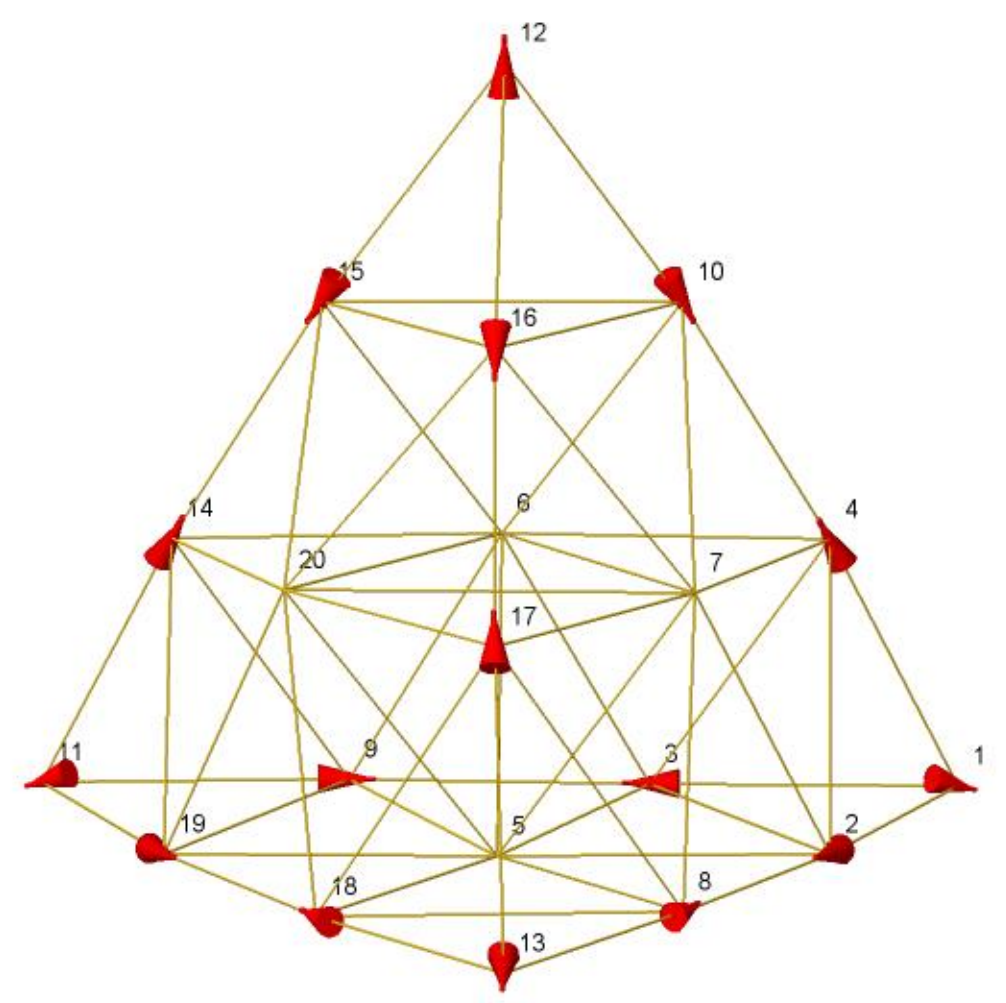

Figure 3.4 Schematic diagram of a highest vibrational mode ( $4^{\text {th }}$ mode of $A_{1}$ symmetry) 


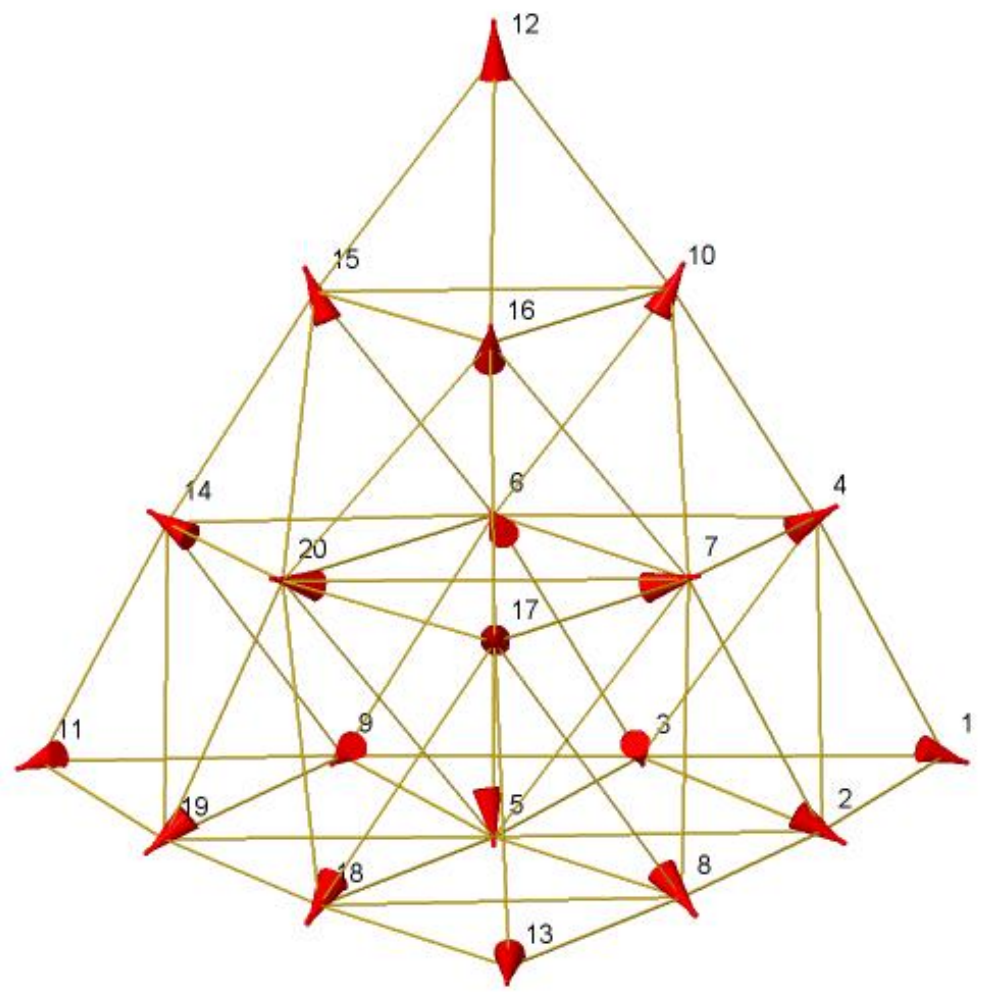

Figure 3.5 Schematic diagram of a Raman breathing mode ( $3^{\text {rd }}$ mode of $A_{1}$ symmetry)
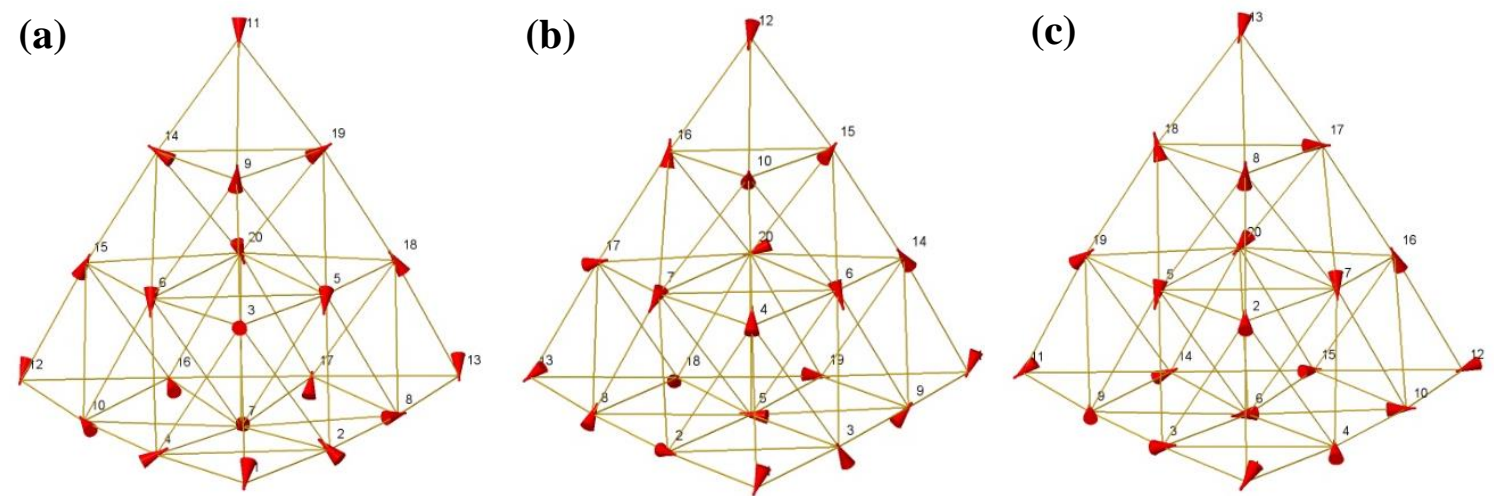

Figure 3.6 Schematic diagram of a degenerate $6^{\text {th }}$ mode of $T_{2}$ symmetry (mode 49,50 and 51) 


\subsection{Phonon spectral density of $\mathrm{Au}_{20}$}

Phonon spectral density is another way to investigate the vibrational spectrum of $\mathrm{Au}_{20}$. The phonon spectral density gives the number of vibrational states that can occupy the phonons per unit volume of crystal per unit energy. ${ }^{14}$ The Fourier transform of the velocity auto-correlation (VAC) function was taken to calculate the phonon spectral density. ${ }^{8}$, 14-16 The Molecular Dynamics (MD) simulations were performed at $300 \mathrm{~K}$ in order to obtain the atomic velocities. The time step was $1 \mathrm{fs}$ and the total duration of the MD simulation was $50 \mathrm{ps}$. The velocity auto-correlation function can be expressed as follows ${ }^{14}$ :

$V A C(t)=\frac{1}{N} \sum_{n=1}^{N} \frac{\left\langle v_{n}(t) \cdot v_{n}(0)\right\rangle}{\left\langle v_{n}(0) \cdot v_{n}(0)\right\rangle}$

where,

$\left\langle v_{n}(t) \cdot v_{n}(0)\right\rangle=\frac{1}{M} \sum_{m=1}^{M} v_{n}\left(t_{m}\right) \cdot v_{n}\left(t_{m}+t\right)$

$\left\langle v_{n}(0) \cdot v_{n}(0)\right\rangle=\frac{1}{M} \sum_{m=1}^{M} v_{n}\left(t_{m}\right) \cdot v_{n}\left(t_{m}\right)$

$t$ is the time, $N$ is the number of atoms and $M$ is the number of time origins (time steps). $v\left(t_{m}\right)$ and $v\left(t_{m}+t\right)$ are the atomic velocities at the reference time and delay time in the simulation respectively. $v_{n}(0)$ is the velocity at the zero correlation time. The phonon spectral density (PDS) given by ${ }^{14}$;

$\operatorname{PDS}(\omega)=\int_{\infty}^{\infty} V A C(t) \cos (\omega t) d t$

(Equation 3.5)

The VAC function was calculated over all 20 atoms in $\mathrm{Au}_{20}$ cluster for all $\mathrm{x}, \mathrm{y}$ and $\mathrm{z}$ directions. Four MD simulations were run in order to reduce the signal to noise ratio that might affect the PDS 
spectrum. Figure 3.7 shows VAC functions for four different MD simulations and Figure 3.8 shows the average VAC function which was used to calculate the PDS spectrum. During the MD simulations, there are strong interatomic repulsive and attractive forces acting on the atoms, but the atomic positions will remain nearly constant. Therefore, the motion of the atoms can be seen as oscillations. The atoms vibrate forward and backward while reversing the velocity at the end of each oscillation. ${ }^{17}$ As a result of this, VAC function shows a strong oscillatory behavior at the beginning and decays eventually.

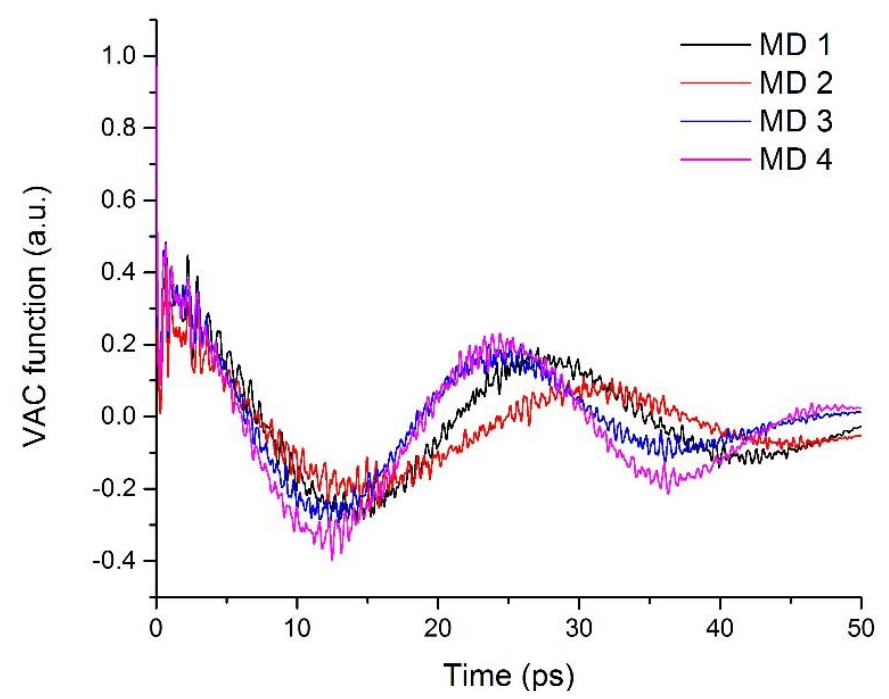

Figure 3.7 VAC function for 4 different MD simulations

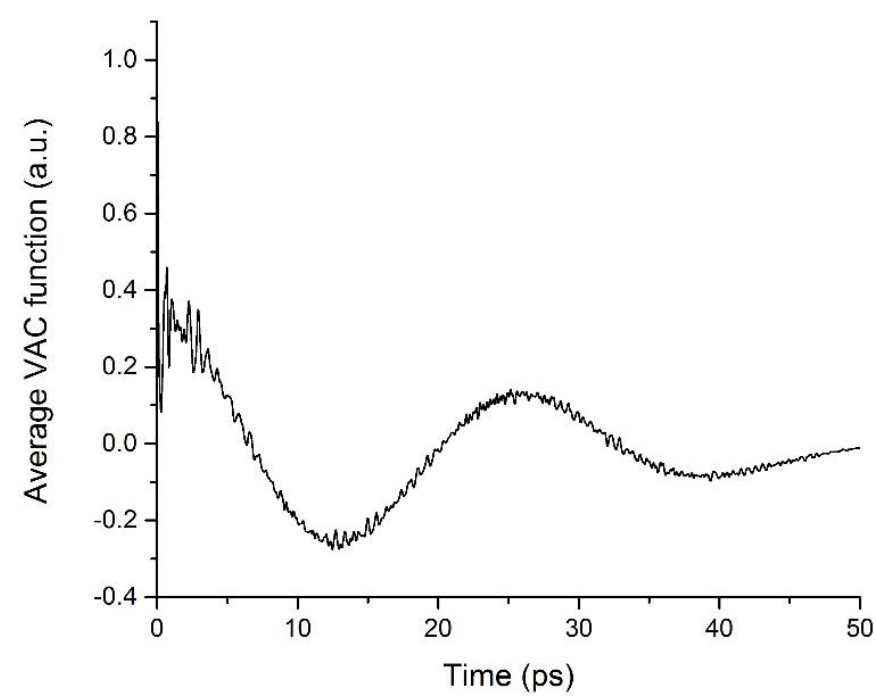

Figure 3.8 Average VAC function which used to calculate the phonon spectral density 
The calculated phonon spectral density spectrum can be seen in Figure 3.9 and it closely agrees with the vibrational modes which were calculated in section 3.3. The most dominant peak can be seen around $46.7 \mathrm{~cm}^{-1}$ and based on the eigen vectors of the vibrational modes it can be identified as the $1^{\text {st }}$ mode of $A_{1}$ symmetry where only the center atoms are displaced perpendicular to the plane.

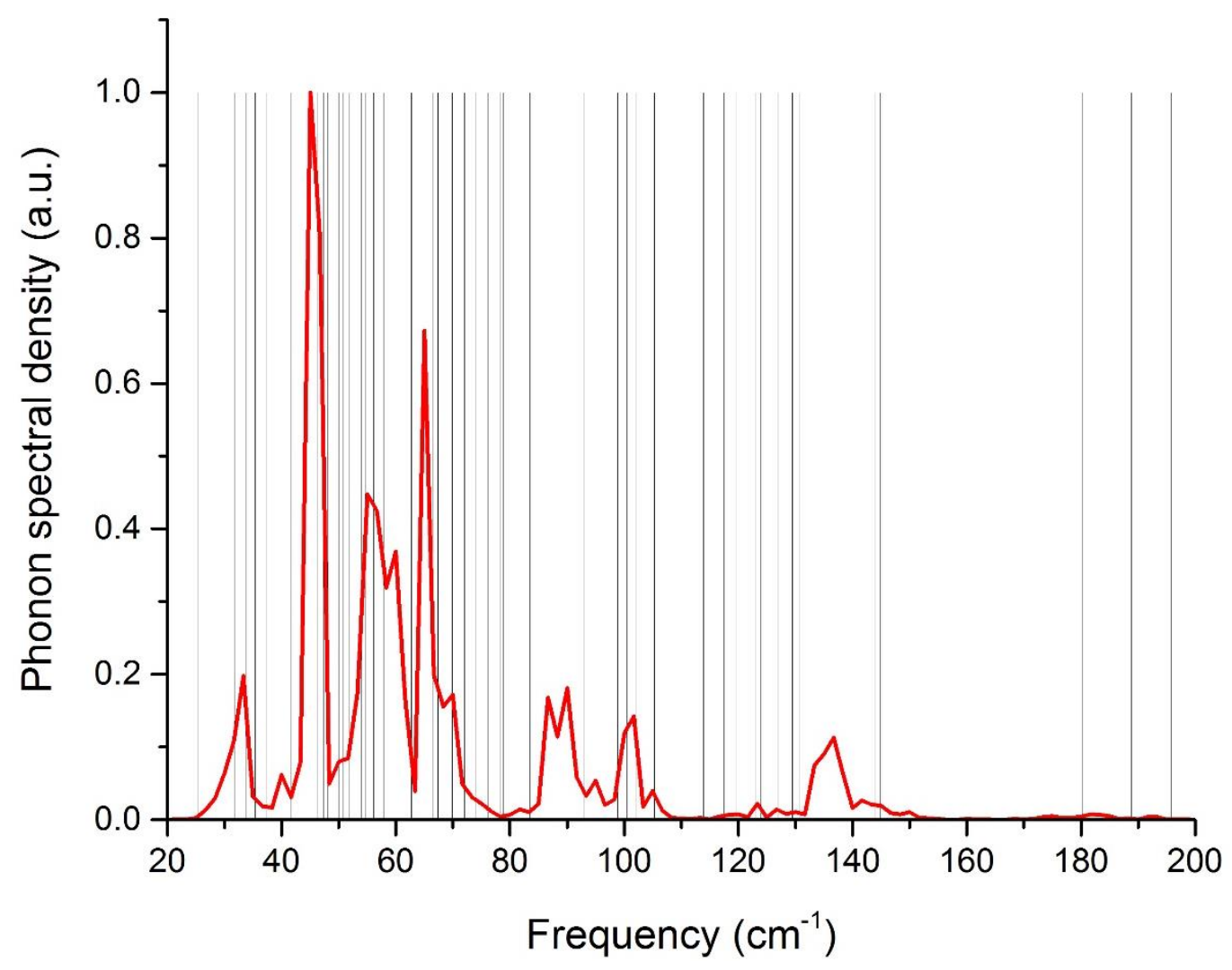

Figure 3.9 Phonon spectral density spectrum of $\mathrm{Au}_{20}$ cluster. Red lines show the distinct peaks 


\subsection{Conclusion}

Most stable tetrahedral structure ${ }^{6}$ for $\mathrm{Au}_{20}$ (among the other possible structures such as icosahedral and octahedral) was used for calculations. The vibrational spectrum of the $\mathrm{Au}_{20}$ cluster was investigated in two different methods (vibrational modes calculations using dynamical matrix and phonon spectral density calculation). The Au-Au bond distances and $\mathrm{Au}-\mathrm{Au}-\mathrm{Au}$ bond angles of optimized structure well agreed with the values reported in literature. The symmetry of some vibrational modes were identified using the eigen vectors. Some discrepancies can be found in this work and previous work probably due to the differences of computational methods. However, there is no experimental work to compare these results yet. 


\section{References}

1. A. Yang, W. Fa and J. Dong, Physics Letters A, 2010, 374, 4506-4511.

2. P. Gruene, D. M. Rayner, B. Redlich, A. F. G. van der Meer, J. T. Lyon, G. Meijer and A. Fielicke, Science, 2008, 321, 674-676.

3. B. Molina, J. R. Soto and A. Calles, Revista mexicana de física, 2008, 54, 314-318.

4. H. S. De, S. Krishnamurty and S. Pal, The Journal of Physical Chemistry C, 2009, 113, 7101-7106.

5. E. S. Kryachko and F. Remacle, International Journal of Quantum Chemistry, 2007, 107, 2922-2934.

6. J. Li, X. Li, H.-J. Zhai and L.-S. Wang, Science, 2003, 299, 864-867.

7. A. Tlahuice-Flores, J Mol Model, 2013, 19, 1937-1942.

8. O. F. Sankey and D. J. Niklewski, Physical Review B, 1989, 40, 3979-3995.

9. P. Jelínek, H. Wang, J. P. Lewis, O. F. Sankey and J. Ortega, Physical Review B, 2005, 71, 235101.

10. J. P. Lewis, P. Jelínek, J. Ortega, A. A. Demkov, D. G. Trabada, B. Haycock, H. Wang, G. Adams, J. K. Tomfohr, E. Abad, H. Wang and D. A. Drabold, physica status solidi (b), 2011, 248, 1989-2007.

11. R. Hanson, Journal of Applied Crystallography, 2010, 43, 1250-1260.

12. Vibrational modes, http://nanosurf.fzu.cz/wiki/doku.php?id=vibrational_modes, Accessed 04/05/2015, 2014.

13. J. C. Slater, Physical Review, 1953, 91, 528-530.

14. D. Raymand, T. J. Jacobsson, K. Hermansson and T. Edvinsson, The Journal of Physical Chemistry C, 2012, 116, 6893-6901. 
15. J. S. Lin, S. P. Ju, W. J. Lee and M. X. Weng, in Nano/Micro Engineered and Molecular Systems, 2006. NEMS '06. 1st IEEE International Conference on, 2006, pp. 1076-1079.

16. F. Kirchhoff, M. J. Mehl, N. I. Papanicolaou, D. A. Papaconstantopoulos and F. S. Khan, Physical Review B, 2001, 63, 195101.

17. The Velocity Autocorrelation Function, http://www.ccp5.ac.uk/DL_POLY/Democritus/Theory/vaf.html, Accessed 04/07/2015. 


\section{Chapter 4}

\section{Phonon-induced relaxation of plasmon excitation in Aus5 clusters}

\subsection{Introduction}

Noble metal nanoparticles have been extensively studied in different areas such as bio-sensing, photocatalysis, nanophotonics, plasmonics, solar cells and data storage for years due to their remarkable optical activity. Surface plasmons are mainly responsible for this remarkable optical response and this is potentially useful for many applications. Therefore it is very important to understand the surface plasmon relaxation dynamics of metal nanoparticles in order to develop novel systems with surface plasmons. Usually relaxation of plasmons via electron-phonon interactions takes from 1 to 10 ps. ${ }^{1}$

The electron density localization of each energy state allows to identify them as bulk, surface or plasmon states. ${ }^{2}$ Guo $\mathrm{et} \mathrm{al}^{2}$ showed the difference between the electron density localization in the $\mathrm{Ag}_{104}$ for bulk, surface and plasmon states. The electron densities of the plasmon states are highly delocalized compared to the bulk and surface states. The delocalization of electron density increases with the energy of the plasmon states. ${ }^{1}$ A nano cluster consists of $55 \mathrm{Au}$ atoms was used in this work in order to investigate the plasmon relaxation dynamics in Au nanoparticles. The $\mathrm{Au}_{55}$ was extensively investigated in both computational and experimental works. ${ }^{3-8}$ Also, the size of $\operatorname{Au}_{55}(\mathrm{~d} \sim 1.4 \mathrm{~nm})$ is a moderate size for the Density Functional Theory (DFT) calculations and gives more reliable data which can be compared with experimental data. ${ }^{4}$ However, electron relaxation dynamics for the $\mathrm{Au}_{55}$ is not well understood. There are two previously proposed 
electron relaxation pathways; molecular like relaxation ${ }^{8}$ and bulk like relaxation ${ }^{7}$ based on the electron relaxation of ligand attached $\mathrm{Au}_{55}$ clusters. Therefore, it is worthwhile to perform a computational investigation for plasmon relaxation dynamics in $\mathrm{Au}_{55}$.

\subsection{Geometrical optimization}

The icosahedral structure ${ }^{4}$ of Auss (Figure 4.1) which is the most stable structure ${ }^{9}$ among the other high symmetry structures such as cuboctahedral and decahedral was used for this work. First, geometrical structure optimization of $\mathrm{Au}_{55}$ was performed at $300 \mathrm{~K}$ using FIREBALL ${ }^{10-12}$; a localorbital DFT package to identify the lowest energy structure. Time step size was 1 fs and RMS cutoff was set as 0.05 . The variation of total energy of the $\mathrm{Au}_{55}$ cluster can be seen in Figure 4.2.

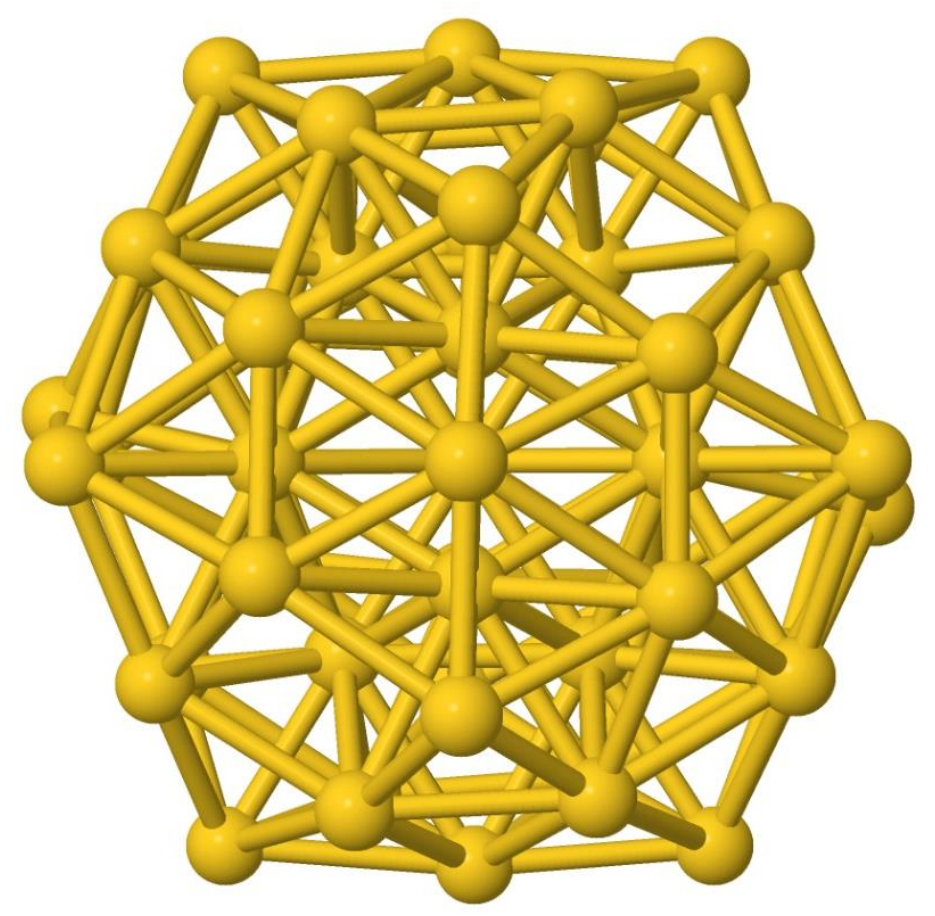

Figure 4.1 Icosahedral structure of $\mathrm{Au}_{55}$ 


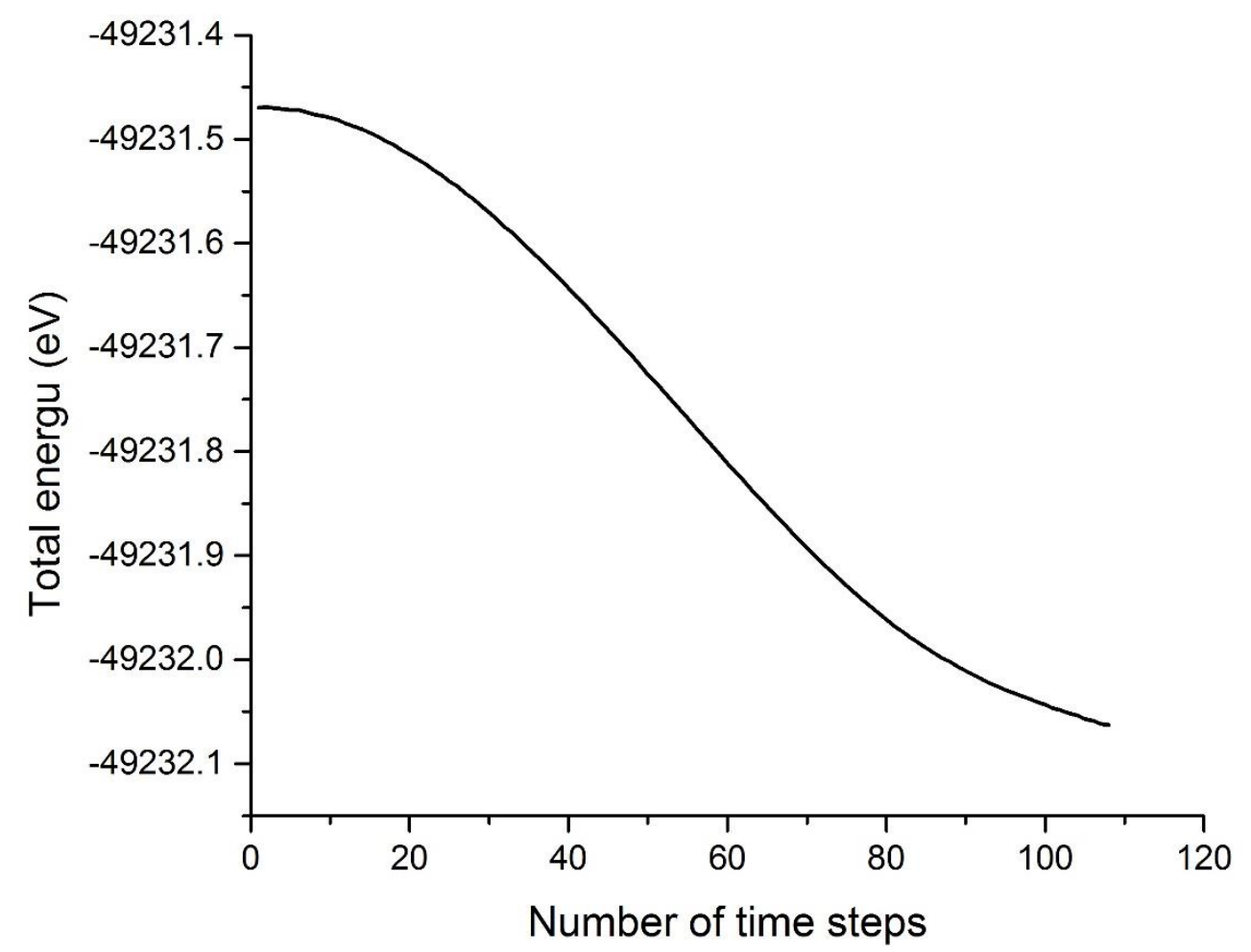

Figure 4.2 The variation of total energy of the $\mathrm{Au}_{55}$ cluster during the geometrical structure optimization

\subsection{Identify the plasmon states}

Electron densities of all the energy states which are above the LUMO (lowest unoccupied molecular orbital) state were investigated in order to identify the highly delocalized plasmon states. The arbitrary state number came from the computational output but they represent consecutive energy states. The difference of the electron density localization between surface states and plasmon states can be clearly seen. Figures 4.3 to 4.5 show the electron densities of HOMO (highest occupied molecular orbital) state, LUMO state and the first state above the LUMO state respectively. Figures 4.6 to 4.10 show five plasmon states which were chosen based on the localized electron densities for this work. According to Figures 4.6 to 4.10, electron densities extended far away with respect to the Au atoms for plasmon states. 


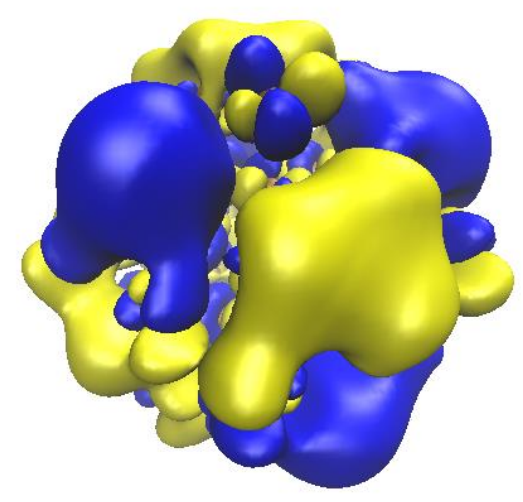

Figure 4.3 HOMO state $\left(\mathrm{S}_{303}\right)$

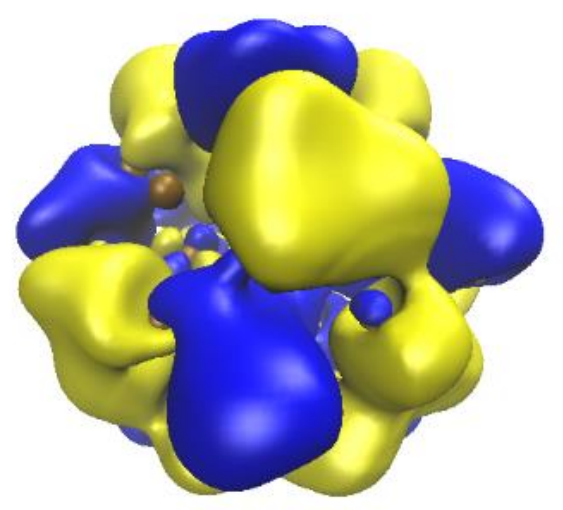

Figure 4.5 State $305\left(\mathrm{~S}_{305}\right)$

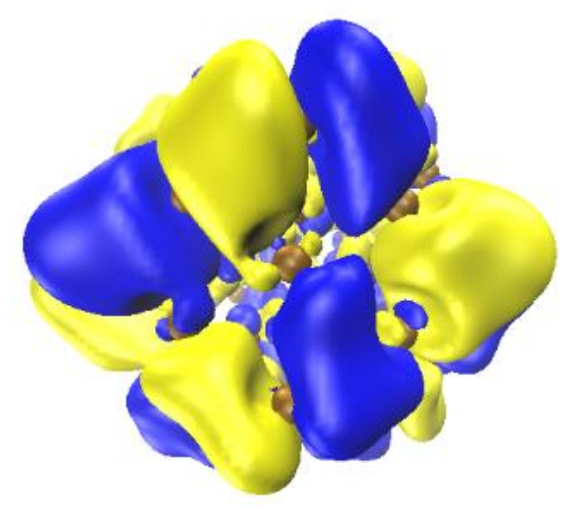

Figure 4.7 Plasmon state $\left(\mathrm{S}_{339}\right)$

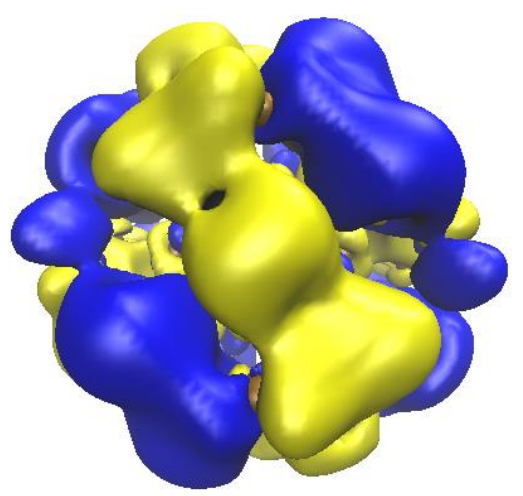

Figure 4.4 LUMO state $\left(\mathrm{S}_{304}\right)$

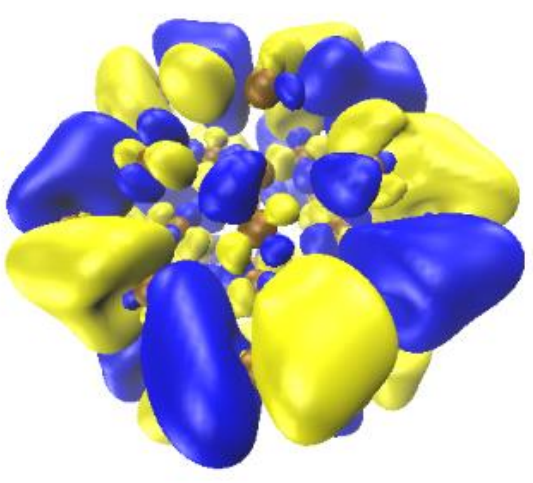

Figure 4.6 Plasmon state $\left(\mathrm{S}_{338}\right)$

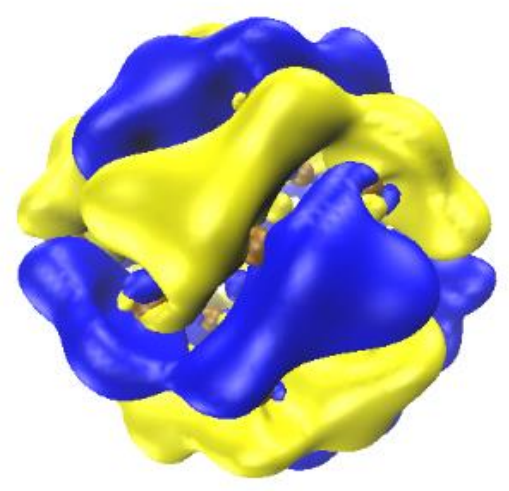

Figure 4.8 Plasmon state $\left(\mathrm{S}_{341}\right)$ 


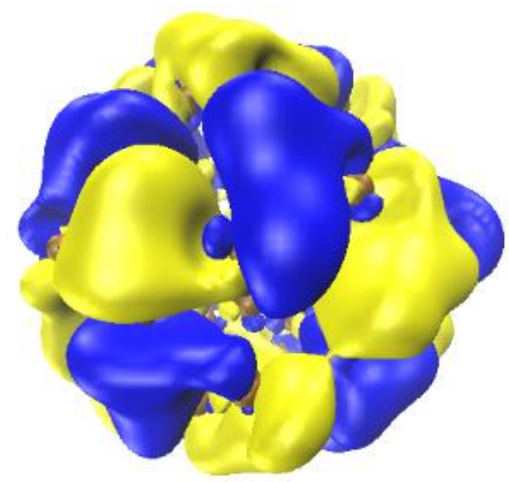

Figure 4.9 Plasmon state $\left(\mathrm{S}_{342}\right)$

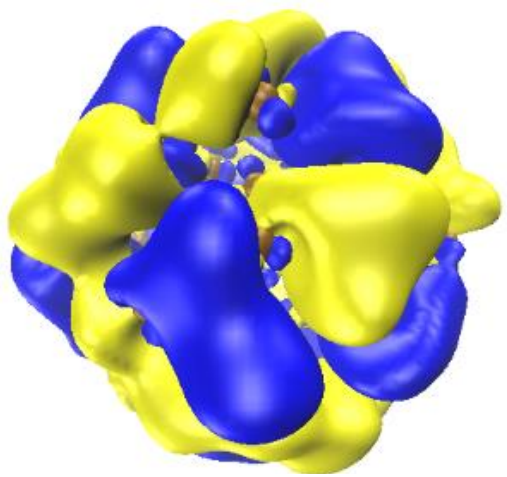

Figure 4.10 Plasmon state $\left(\mathrm{S}_{343}\right)$

The energies of the HOMO state $\left(\mathrm{S}_{303}\right)$ and LUMO state $\left(\mathrm{S}_{304}\right)$ were found to be $-3.85 \mathrm{eV}$ and $3.25 \mathrm{eV}$ respectively. Table 4.1 shows the energies of the 5 plasmon states which were investigated in this work. The position of the energy eigen value was used as the state number. Figure 4.11 show the locations of each HOMO, LUMO and 5 plasmon states along with the density of states of $\mathrm{Au}_{55}$.

Table 4.1. Energy of each initial plasmon state

\begin{tabular}{|c|c|}
\hline Plasmon state & Energy (eV) \\
\hline $\mathrm{S}_{338}$ & -0.2116 \\
\hline $\mathrm{S}_{339}$ & -0.1820 \\
\hline $\mathrm{S}_{341}$ & +0.3973 \\
\hline $\mathrm{S}_{342}$ & +0.6832 \\
\hline $\mathrm{S}_{343}$ & +0.8090 \\
\hline
\end{tabular}




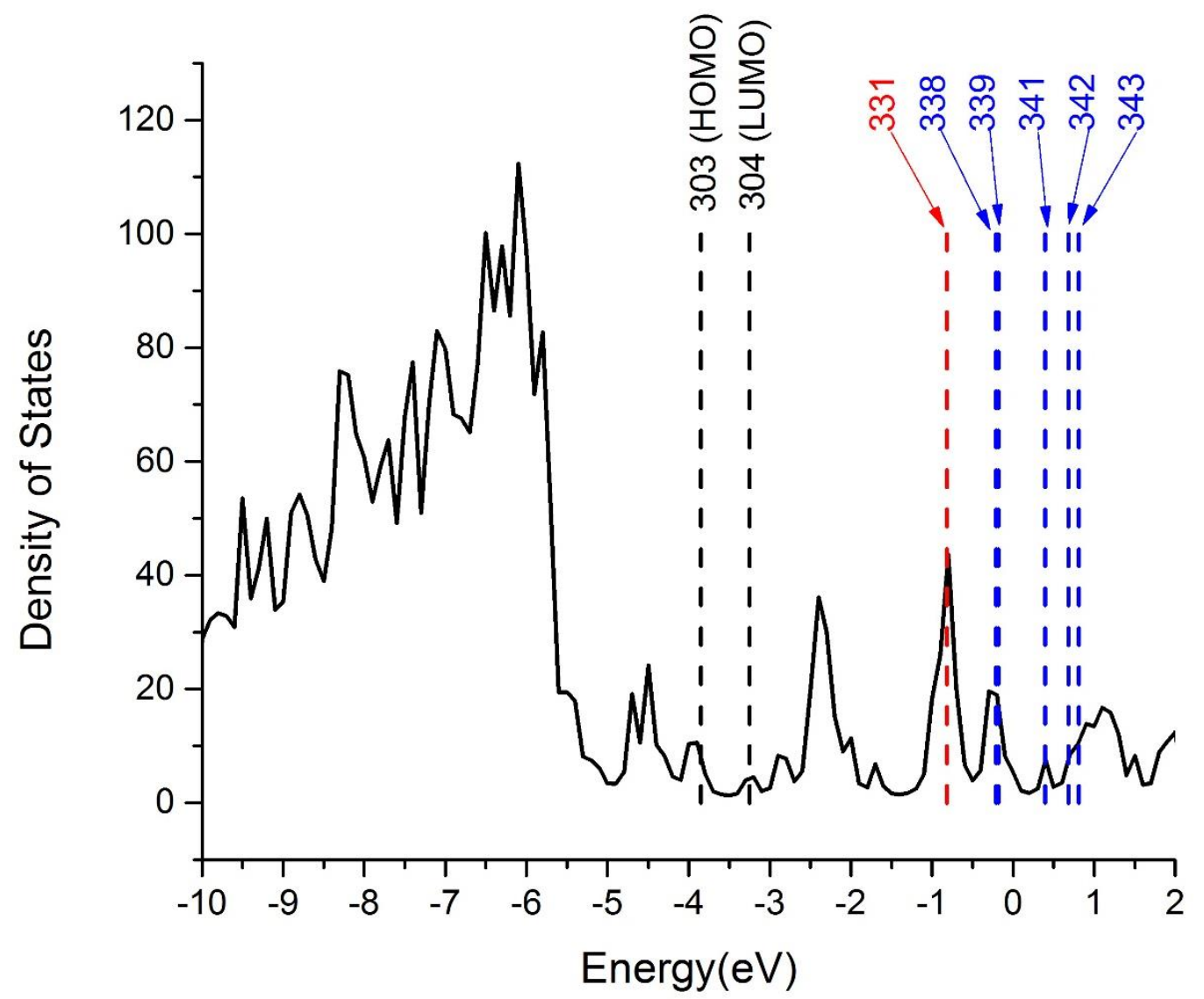

Figure 4.11 Density of states of $\mathrm{Au}_{55}$ and energy of plasmon states

\subsection{Non-adiabatic molecular dynamics (NAMD) simulations}

\subsubsection{Computational method}

For non-adiabatic molecular dynamics (NAMD) simulations, it is necessary to keep the inter relation between nuclear motion and electronic quantum state while atoms follow the classical trajectories. ${ }^{13}$ The time dependent Schrodinger equation was used to explain the time evolution of 
the electronic wave function. ${ }^{13}$ Usually single particle Hartree Fock (HF) or Kohn-Sham (KS) orbitals are used to solve the time dependent Schrodinger equation. ${ }^{13}$ The different potential energy surfaces (PES) can be calculated using these single particle orbitals. ${ }^{13}$

The time evolution of single particle $\mathrm{KS}$ orbital $^{13}$ can be written as:

$\widehat{H}_{K S} \varphi_{P}=i \hbar \frac{\partial \varphi_{P}}{\partial t}$

$\widehat{H}_{K S}(R) \psi_{i}=\varepsilon_{i}(R) \psi_{i}$

is the single particle KS Hamiltonian and

$\varphi_{P}(r, t)=\sum_{i} a_{i}(t) \psi_{i}(r, R)$

is the time evolving KS orbitals. The $a_{i}(t)$ in the Equation 4.3, can calculate using the equation:

$\frac{\partial a_{i}(t)}{\partial t}=a_{i}(t) \varepsilon_{i}(R)-i \hbar \sum_{j} a_{j}(t) d_{i j} . V$

where $d_{i j} . V$ represent the coupling between classical motion of the nuclei and the electronic quantum state. $^{13}$

$d_{i j} . V=\sum_{\alpha} d_{i j}^{\alpha} V_{\alpha}$

(Equation 4.5)

where $d_{i j}^{\alpha} \equiv\left\langle\psi_{i} \mid \frac{\partial \psi_{i}}{\partial R_{\alpha}}\right\rangle$ are the non-adiabatic coupling vectors (NACV) between the single particle

KS states ${ }^{13}$ and $V_{\alpha}=\frac{\partial R_{\alpha}}{\partial t}$ is the atomic velocity. ${ }^{14}$ 
Usually, Ehrenfest dynamics ${ }^{15,16}$ and surface hopping ${ }^{17,18}$ are used for NAMD simulations. The surface hopping method is better than the Ehrenfest method where changes in electronic populations affects the nuclear motion. The fewest switches surface-hopping (FSSH) algorithm ${ }^{17}$ was used for NAMD methodology. The hopping probability depends on both non-adiabatic coupling vector and the atomic velocity. ${ }^{14}$ NACV's are calculated during the simulation using the previously implemented efficient algorithm. ${ }^{13}$ During the electronic transitions, nuclear velocities were rescaled to conserve the energy. ${ }^{14}$ If the kinetic energy present is sufficient, hopping in to the higher energy state is possible. As a result of this, it is possible to see up and down electron transition among the energy states during the NAMD simulations.

\subsubsection{NAMD simulations}

An electron was removed from the HOMO state and placed it in one of the 5 plasmon states in order to simulate the plasmon excitation. The excited electron relaxes during the NAMD simulation which was performed for 10,000 time steps at $300 \mathrm{~K}$. The total simulation time was 30 ps (time step size is $3 \mathrm{fs}$ ). Fifty NAMD trajectories were performed in order to reduce the error for each plasmon excitation. Random initial coordinates and initial velocities were generated using an automated python script.

The electron population of each energy state between the specific plasmon state and HOMO state were extracted for all the 50 NAMD trajectories and the average was used to generate the population plots (Figures 4.12 to 4.16). For an example, there were 36 energy states involved in electron relaxation from the $S_{338}$ (plasmon state) to $S_{303}$ (HOMO state). Similarly 37, 39, 40 and 41 energy states were involved for electron relaxation from the states of $S_{339}, S_{341}, S_{342}$ and $S_{343}$ in 
to the state $\mathrm{S}_{303}$. Figures 4.12 to 4.16 show the variations of average population of all the energy states which were involved for the electron relaxation from each plasmon state. It was found that state $S_{331}$ highly populated during the electronic relaxation process regardless of the energy of the initial plasmon state. The HOMO state in black color, initial plasmon state in blue color and state $S_{331}$ in red color can be seen. The populations of the other states were faded for clarity of the major states.

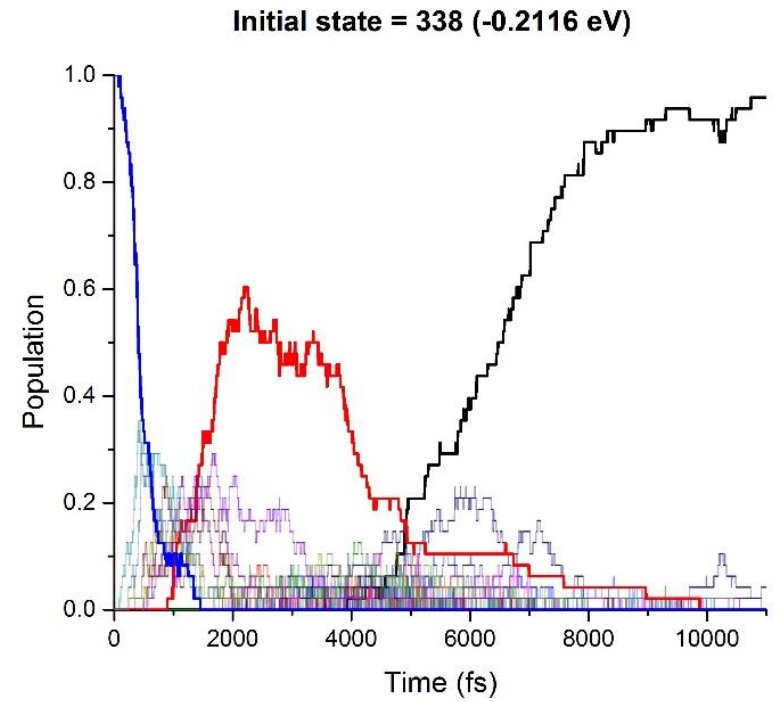

Figure 4.12 The populations of all the states between HOMO state and initial plasmon state $\mathrm{S}_{338}$

Initial state $=341(+0.3973 \mathrm{eV})$

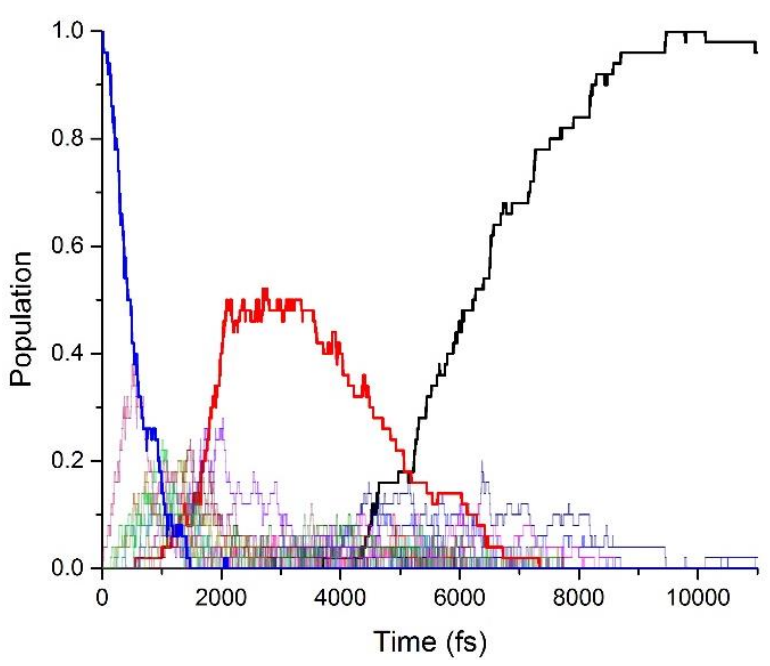

Figure 4.14 The populations of all the states between HOMO state and initial plasmon state $\mathrm{S}_{341}$

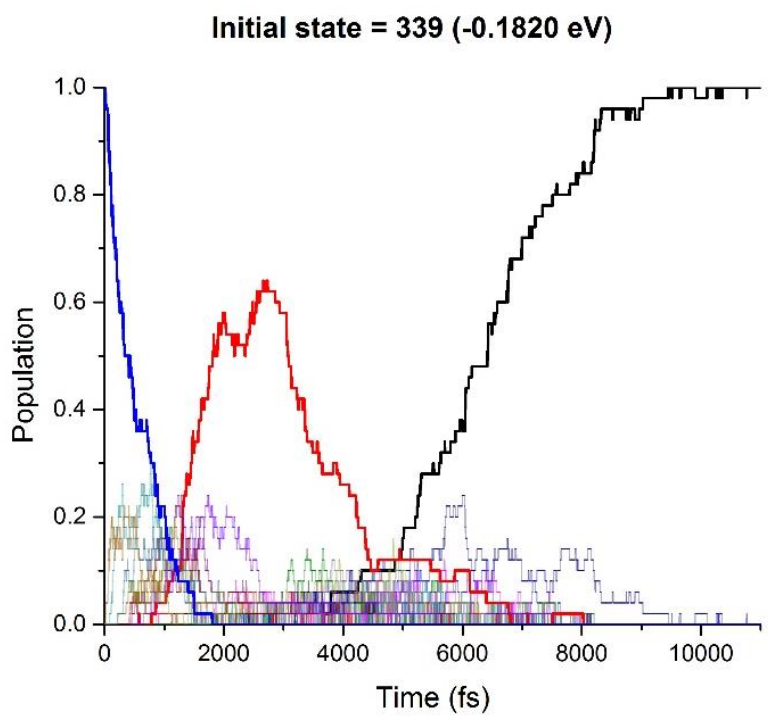

Figure 4.13 The populations of all the states between HOMO state and initial plasmon state $\mathrm{S}_{339}$

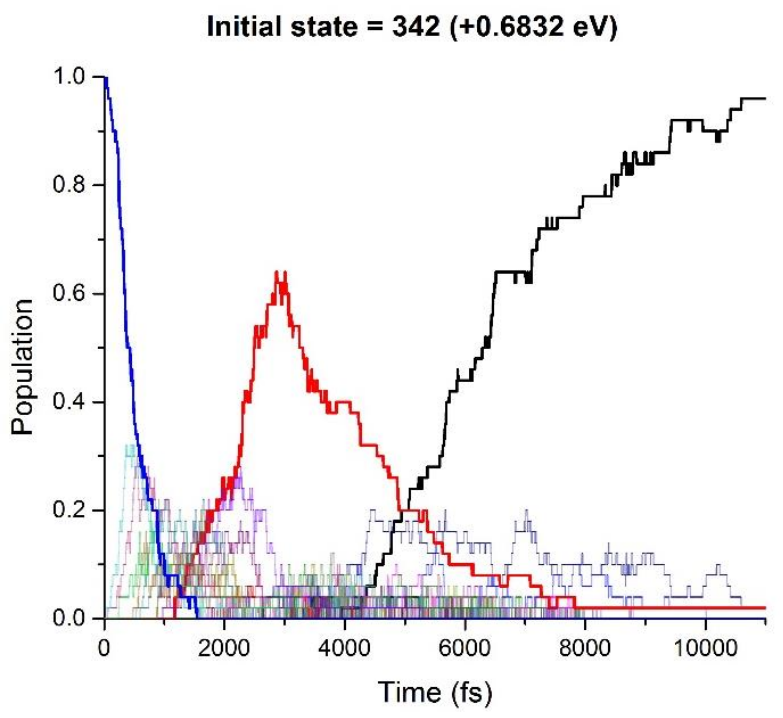

Figure 4.15 The populations of all the states between HOMO state and initial plasmon state $S_{342}$ 


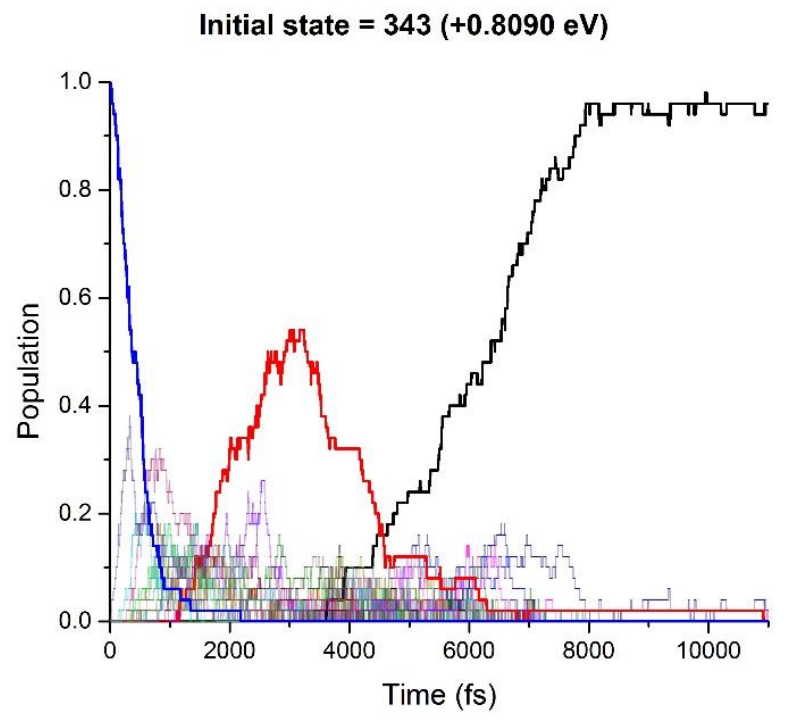

Figure 4.16 The populations of all the states between HOMO state and initial plasmon state $S_{343}$

The maximum average population (from 50 trajectories) of each energy state (except HOMO state and plasmon state) were plotted as a function of state number (Figure 4.17).

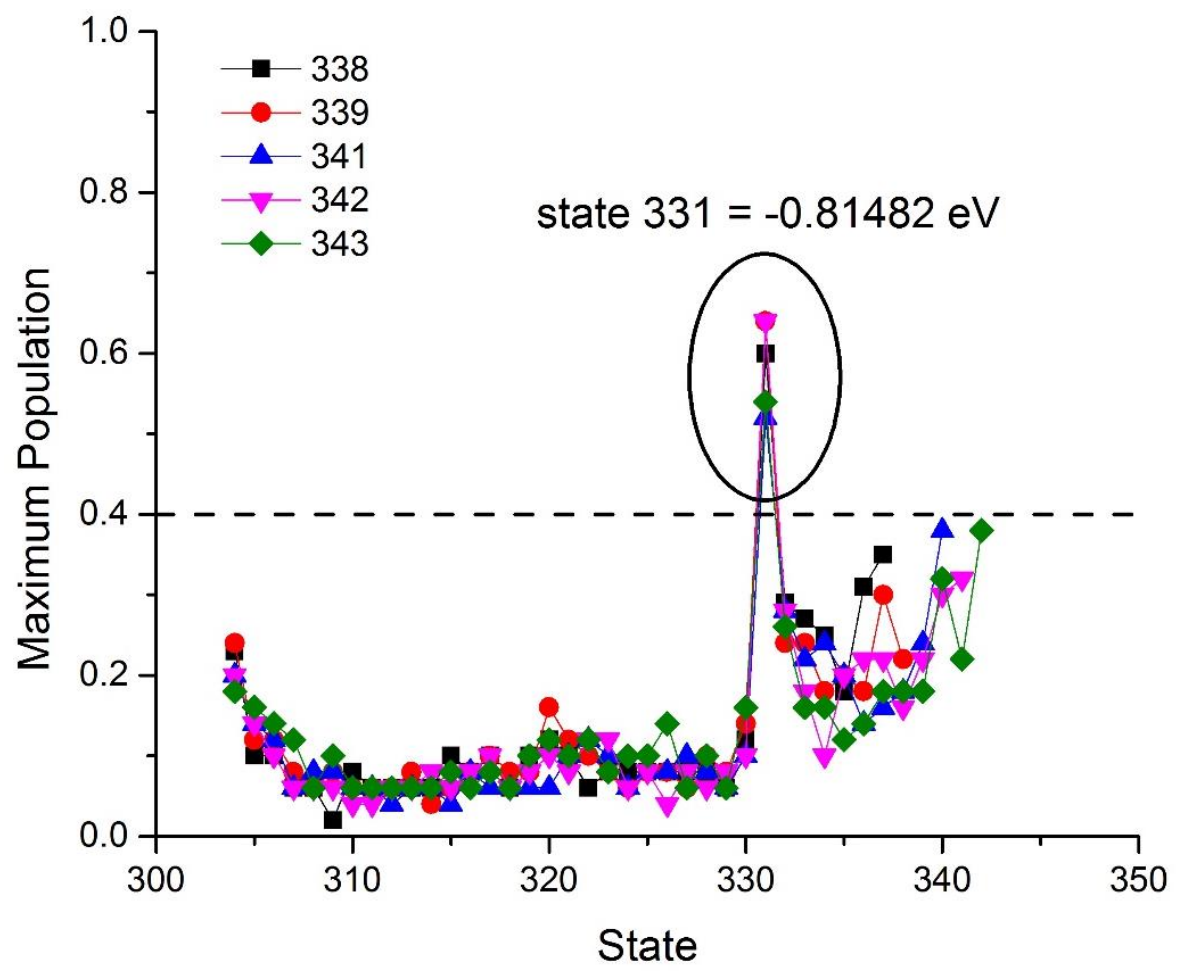

Figure 4.17 Average maximum populations at each state for five different initial plasmon states 
According to the Figure 4.17, state $\mathrm{S}_{331}$ at $-0.8148 \mathrm{eV}$ shows the dominant maximum average population compared to the other states regardless of the energy of the initial plasmon state. Then, the populations of the state $S_{331}$ were investigated and it was found that the population remain above 0.4 for more than 1 ps during the relaxation from all 5 initial plasmon states (Figures 4.18 to 4.22). Also, it is very clear to identify the average population variation of highly populated long living state $S_{331}$ in Figures 4.12 to 4.16 (in red color). The average population variation of initial plasmon state, $\mathrm{S}_{303}$ (HOMO state) and state $\mathrm{S}_{331}$ showed a similar pattern among all the 5 initial plasmon states. No other energy state showed a higher average population or a long living during the relaxation (Figures 4.12 to 4.17 ).

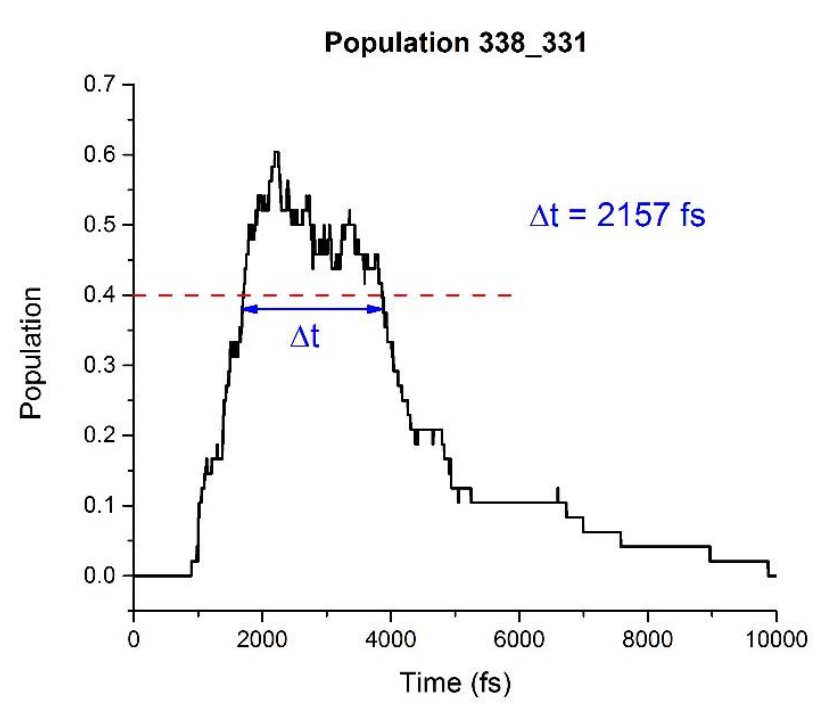

Figure 4.18 The populations of state $S_{343}$ for initial plasmon state $\mathrm{S}_{338}$

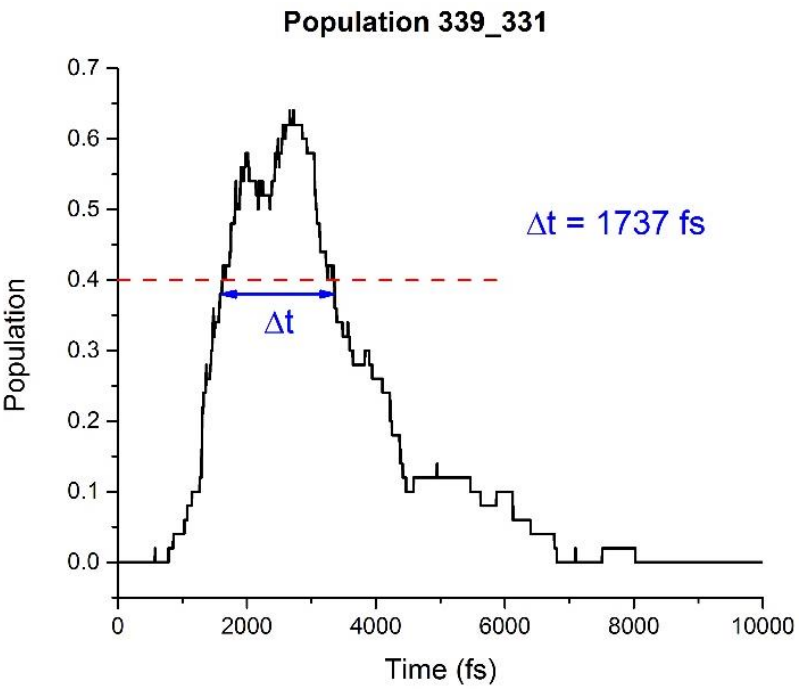

Figure 4.19 The populations of state $S_{343}$ for initial plasmon state $S_{339}$ 


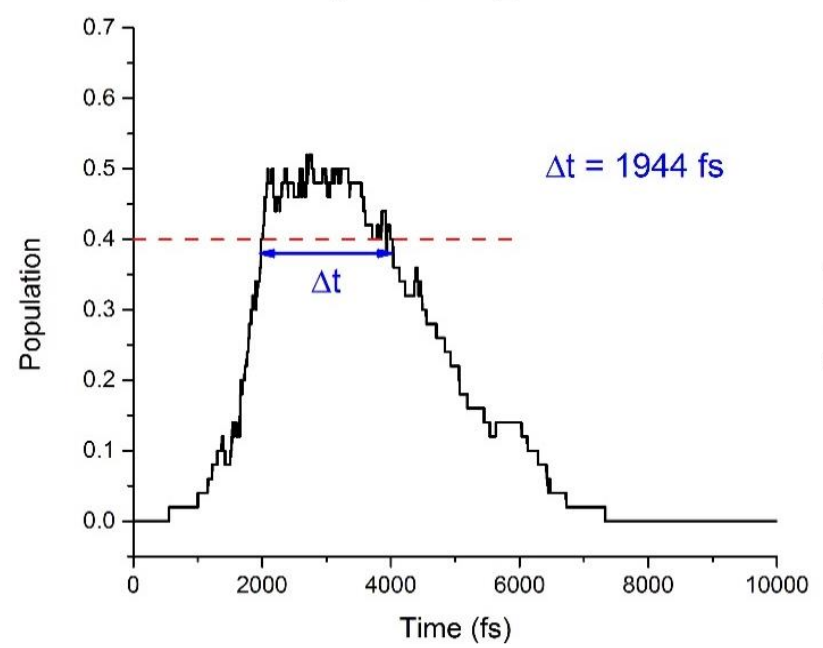

Figure 4.20 The populations of state $S_{343}$ for initial plasmon state $S_{341}$

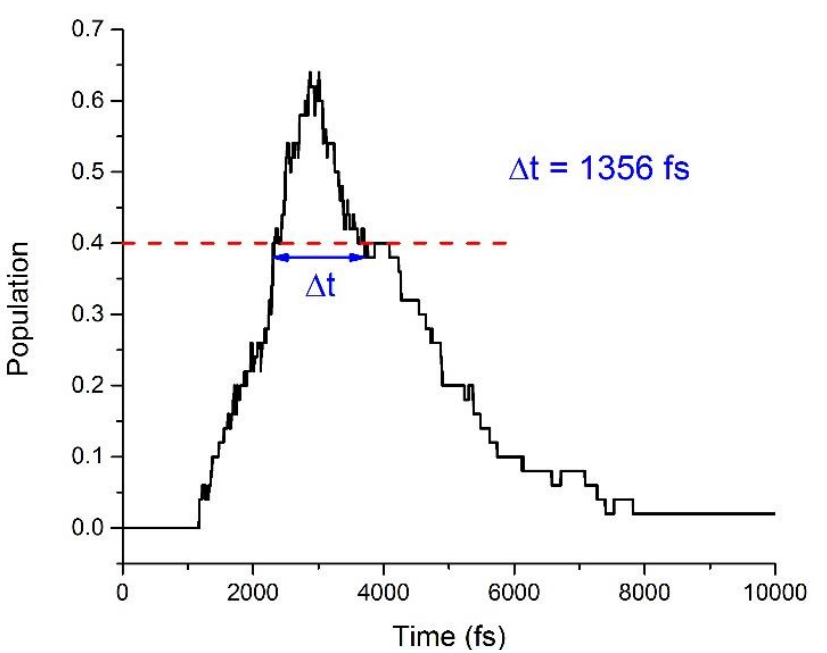

Figure 4.21 The populations of state $S_{343}$ for initial plasmon state $S_{342}$

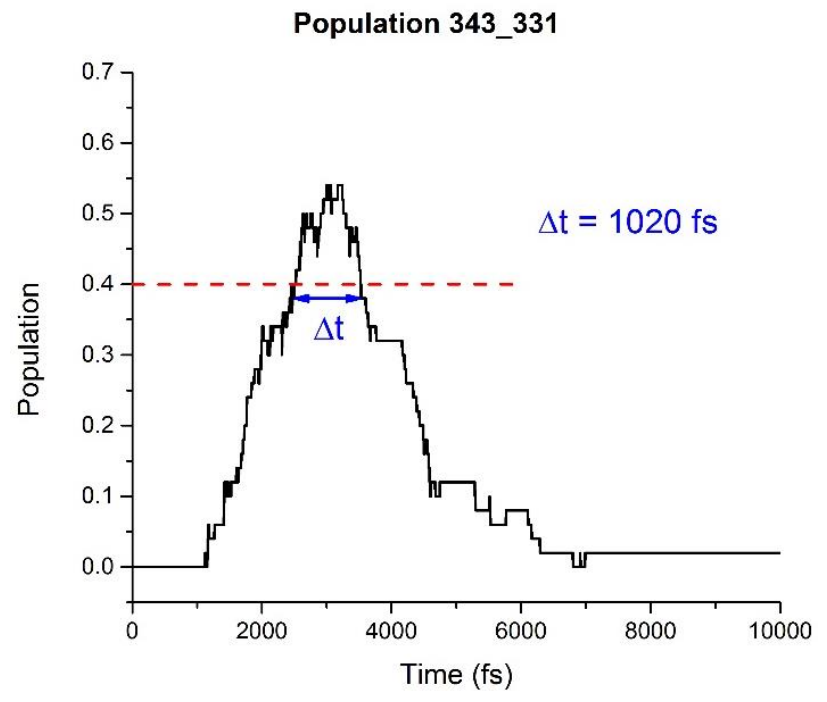

Figure 4.22 The populations of state $S_{343}$ for initial plasmon state $S_{343}$

The overall electronic relaxation can be identified as a 3 step process. First, the excited electron was relaxed in to the state $S_{331}$ from initial plasmon state through the other states in $\sim 1$ ps regardless of the energy of the initial plasmon state. Then, it stayed in state $S_{331}$ for $\sim 3$ ps and after that it gradually relaxed to the HOMO state through the other states. The lack of a conical 
intersection ${ }^{14}$ between state $S_{331}$ and state $S_{330}$ in first $\sim 4$ ps might be the possible reason for the long living of the electron at state $S_{331}$ during the relaxation process. Even if there is a conical intersection between state $S_{331}$ and state $S_{330}$, the probability of electron hopping depends on the both non-adiabatic coupling vector and the atomic velocity (Equation 4.5). ${ }^{14}$ The relaxation was almost completed in $\sim 11$ ps for all the 5 initial plasmon states.

\subsubsection{Conical intersections and electron hopping}

The evolution of energies in states $S_{332}, S_{331}$ and $S_{330}$ along a single trajectory were investigated in order to understand the electronic transition (hopping) to states $S_{331}$ and from $S_{331}$. For an example, Figure 4.23 shows evolution of energies in states $S_{332}, S_{331}$ and $S_{330}$ for one of the trajectories of initial plasmon state $S_{338}$. The electron hop in to the state $S_{331}$ at the 1434 fs and stays there for around $1600 \mathrm{fs}$. There is a short term (66 fs) transition to state $\mathrm{S}_{332}$ that can be seen at $3075 \mathrm{fs}$. At $4041 \mathrm{fs}$, electron hop in to the state $S_{330}$. There is no conical intersection between state $S_{331}$ and state $S_{330}$ for the NAMD simulation time range from 1434 fs to 4041 fs. In Figure 4.23, the electron stay $3-3.5 \mathrm{ps}$ in state $\mathrm{S}_{331}$ which is a good representation for all 50 trajectories. The electronic transitions happen almost at the conical intersections. However, it is not necessary to have an electronic transition at the conical intersection because hopping probability is not always $100 \%$.

Over $90 \%$ of trajectories relaxed through the state $S_{331}$ and more than $75 \%$ of them hop in to state $S_{330}$ from state $S_{331}$. This is valid for all five initial plasmon states. 


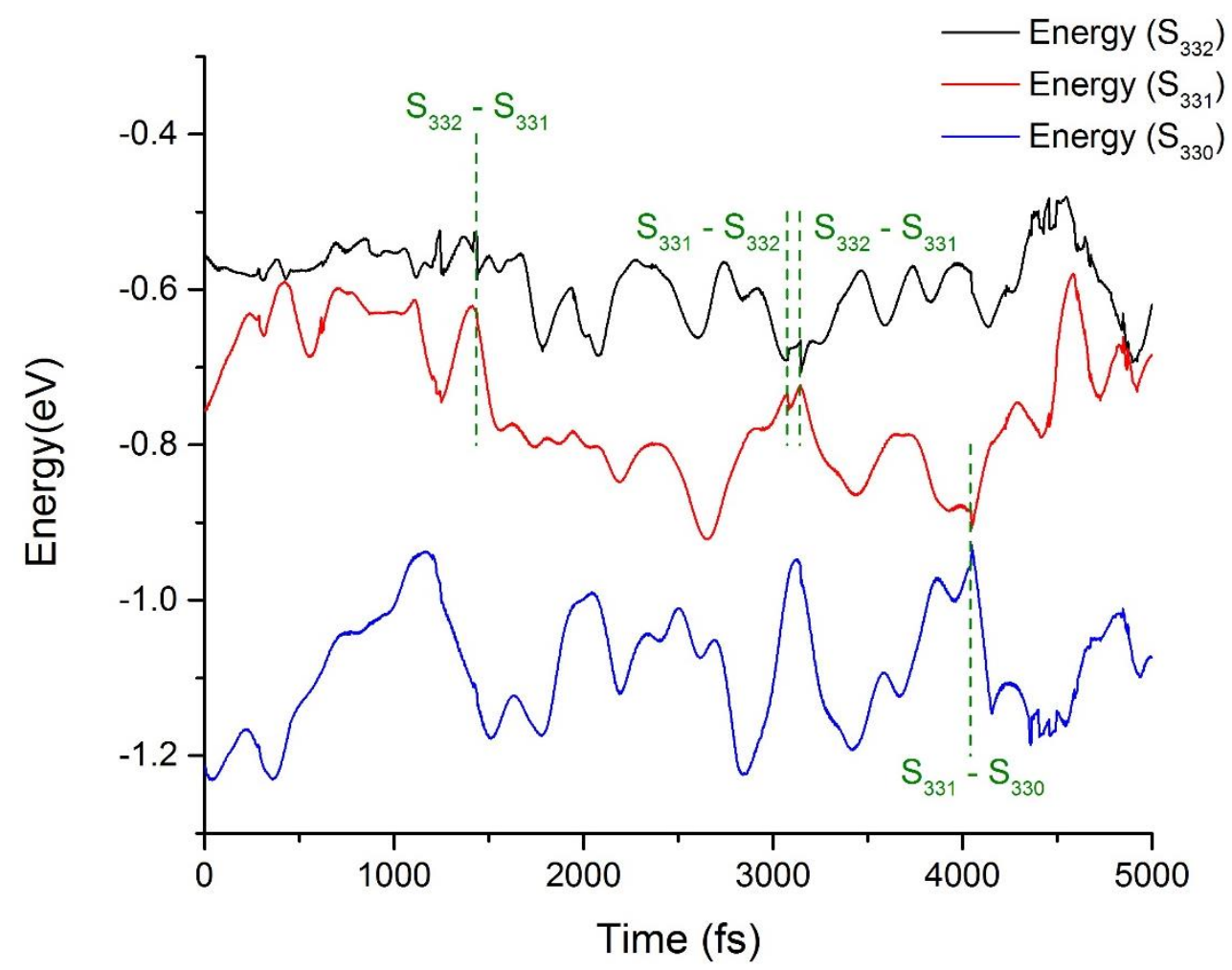

Figure 4.23 The evolution of energies of state $S_{332}, S_{331}$ and $S_{330}$ along one trajectory of initial plasmon state $\mathrm{S}_{338}$

\subsection{Plasmon relaxation time}

Since there are 3 main steps (initial state to state $S_{331}$, stay at state $S_{331}$ and state $S_{331}$ to HOMO state) in the overall relaxation process, the best way is to investigate the [1-population of HOMO state] in order to calculate the total relaxation time constant. The relaxation time constant of [1$S_{\text {номо }}\left(\tau_{0}\right)$ and duration of staying at 1 of [1- $S_{\text {номо }}$ curve $\left(\mathrm{t}_{0}\right)$ were used to calculate the total relaxation time constant. The relaxation time constants of [1- $S_{\text {номо }}\left(\tau_{0}\right)$ were obtained by fitting the population data in to the following Gaussian function ${ }^{1}$.

$f(t)=A \exp \left[-(t / \tau)^{2}\right]$

(Equation 4.6) 
The normalization constant $A$ was fixed to the first population value after plateau at 1 for [1$S_{\text {HOMO }}$ curve. An example Gaussian fit can be seen in Figure 4.24. The [1- $S_{\text {HOMO }}$ data can be seen in black dots and Gaussian fit can be seen in red line.

\section{$\left[1-S_{\text {номо }}\right]($ Initial plasmon State $=338)$}

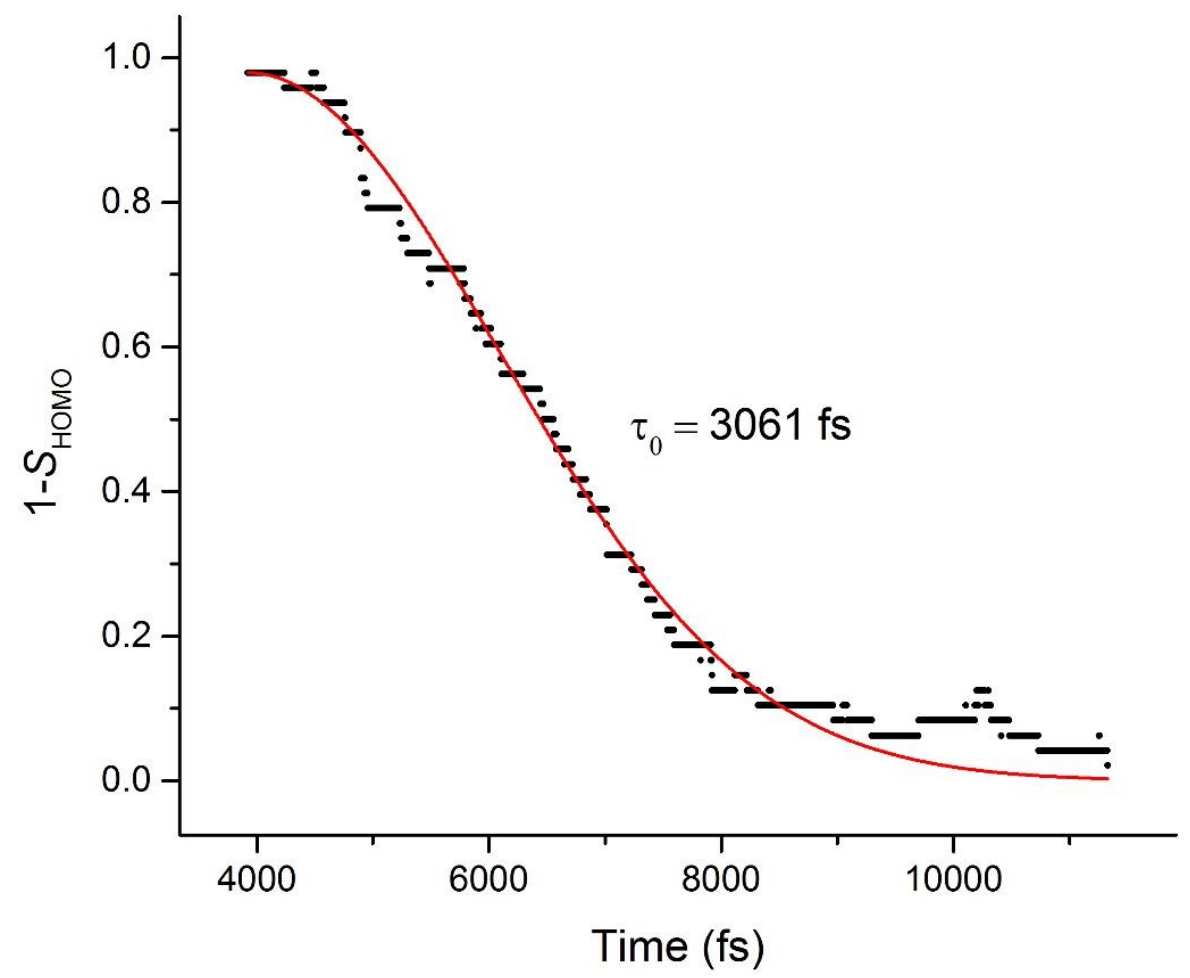

Figure 4.24 The Gaussian fit for the $\left[1-S_{\text {Hомо }}\right]$ data

Figures 4.25 to 4.29 show the relaxation time constants of [1- $S_{\text {HOMO }}\left(\tau_{0}\right)$ and the durations of staying at 1 of $\left[1-S_{\text {Hомо }}\right]$ curve $\left(t_{0}\right)$ for all the initial plasmon states. The durations of staying at 1 of [1- $S_{\text {HOMo }}$ curve $\left(\mathrm{t}_{0}\right)$ is always greater than $3.6 \mathrm{ps}$. In other words, any of the trajectories did not relax in to the HOMO state during the first 3.6 ps of NAMD simulations due to the long living state $S_{331}$. 


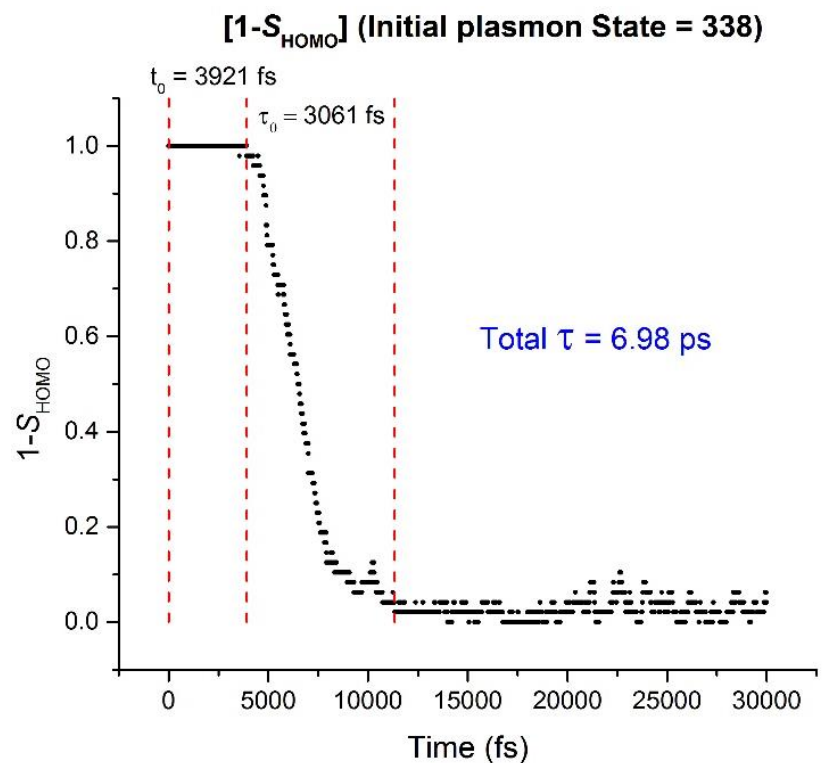

Figure 4.25 Relaxation time constants for initial plasmon state $S_{338}$

$\left[1-S_{\text {номо }}\right]($ Initial plasmon State $=341)$

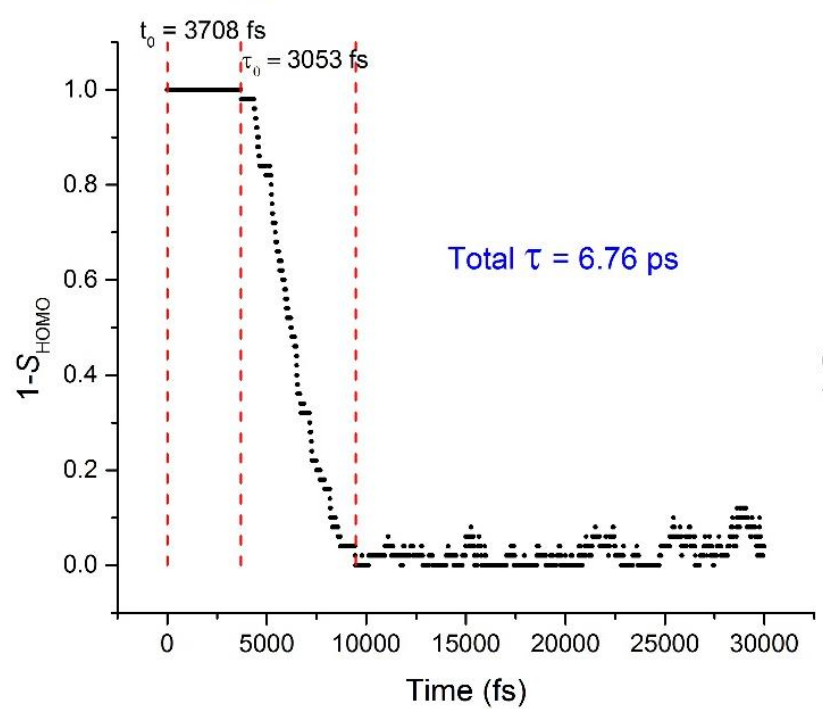

Figure 4.27 Relaxation time constants for initial plasmon state $S_{341}$
$\left[1-S_{\text {номо }}\right]$ (Initial plasmon State $\left.=339\right)$

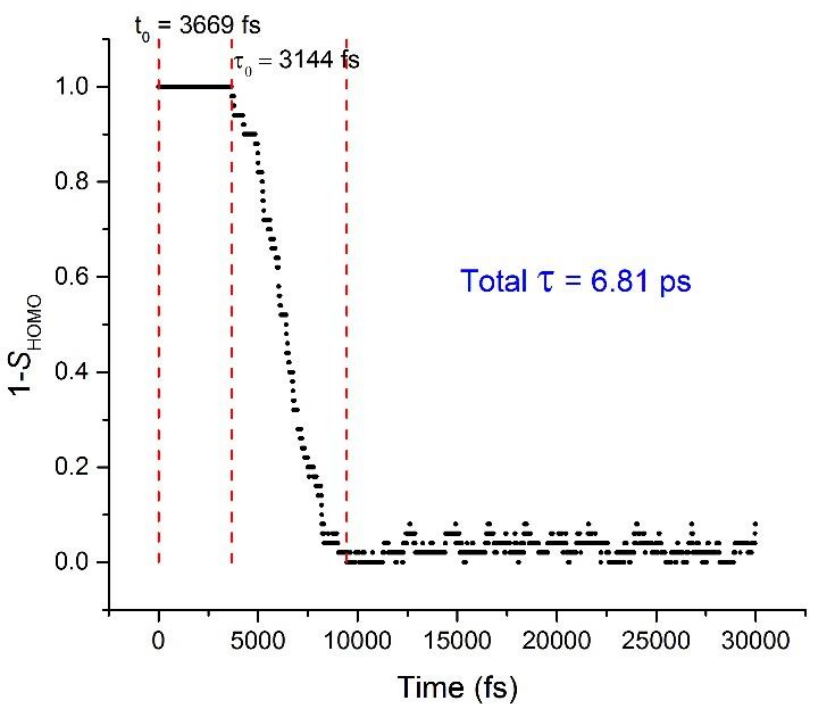

Figure 4.26 Relaxation time constants for initial plasmon state $\mathrm{S}_{339}$

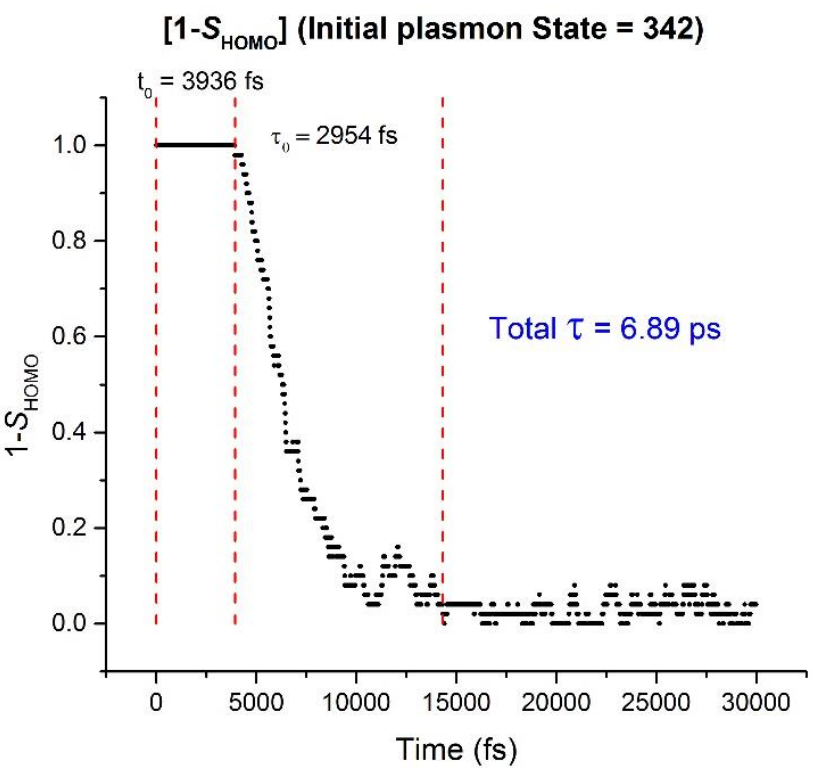

Figure 4.28 Relaxation time constants for initial plasmon state $\mathrm{S}_{342}$ 


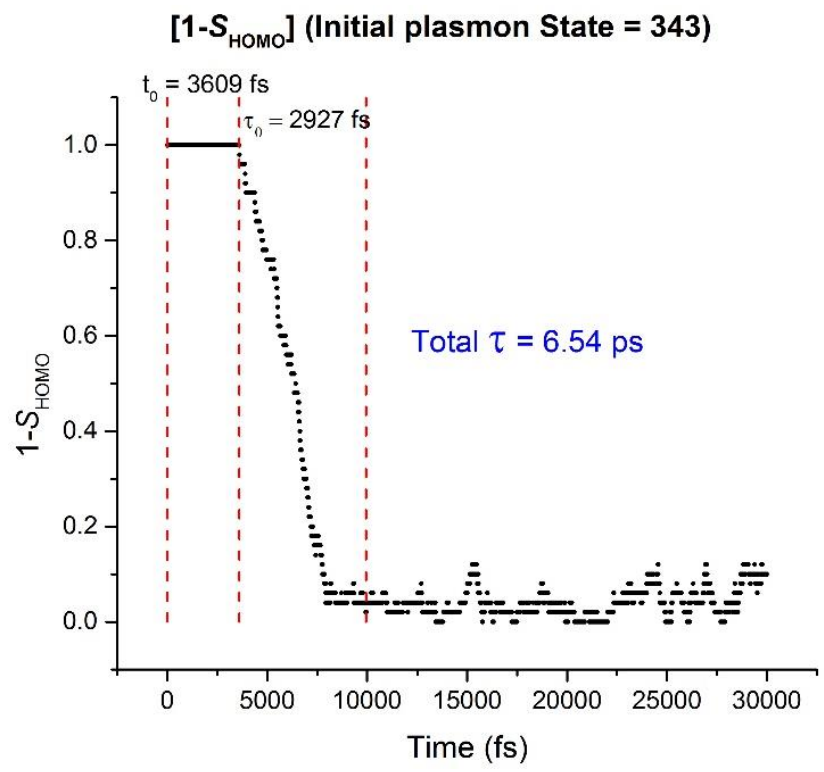

Figure 4.29 Relaxation time constants for initial plasmon state $\mathrm{S}_{343}$

The calculated relaxation time constants for all the initial plasmon states can be found in Table 4.2.

Table 4.2 Summary of the relaxation time constants

\begin{tabular}{|c|c|c|c|}
\hline \multirow{2}{*}{$\begin{array}{c}\text { Initial } \\
\text { plasmon state }\end{array}$} & \multicolumn{3}{|c|}{ Relaxation time constants (ps) } \\
\cline { 2 - 4 } & $\mathbf{t}_{\mathbf{0}}$ & $\boldsymbol{\tau}_{\mathbf{0}}$ & $\tau_{\text {TOT }}$ \\
\hline $\mathbf{3 3 8}$ & 3.92 & 3.06 & $\mathbf{6 . 9 8}$ \\
\hline $\mathbf{3 3 9}$ & 3.67 & 3.14 & $\mathbf{6 . 8 1}$ \\
\hline $\mathbf{3 4 1}$ & 3.71 & 3.05 & $\mathbf{6 . 7 6}$ \\
\hline $\mathbf{3 4 2}$ & 3.94 & 2.95 & $\mathbf{6 . 8 9}$ \\
\hline $\mathbf{3 4 3}$ & 3.61 & 2.93 & $\mathbf{6 . 5 4}$ \\
\hline
\end{tabular}


According to the Table 4.2, the overall relaxation time constant was found to be $\sim 6.8 \mathrm{ps}$ and it is independent of the energy of the initial plasmon state. This $\mathrm{Au}_{55}$ electron relaxation dynamics are very different compared to the previously reported electron relaxation dynamics of $\mathrm{Ag}_{68}$ clusters. $^{1}$ According to Neukirch et $a l^{1}$ the relaxation time constant depends on the energy of the initial plasmon state for $\mathrm{Ag}_{68}$. For high energy plasmon excitation in $\mathrm{Ag}_{68}$, the electron stays a longer time in low energy plasmon states before relaxes in to the bulk states. But for low energy plasmon excitation, the electron quickly relaxes in to the bulk states. There is a clear difference between the relaxation time constants of high energy $(\tau=1.21 \mathrm{ps})$ and low energy $(\tau=0.68 \mathrm{ps})$ plasmon excitations in $\mathrm{Ag}_{68}{ }^{1}$ In $\mathrm{Ag}_{68}$, the energy difference between the investigated lowest energy plasmon state and highest energy plasmon state was $\sim 0.3 \mathrm{eV}$. However in this work for $\mathrm{Au}_{55}$, the energy difference between the state $S_{338}$ (lowest energy plasmon state) and $S_{343}$ (highest energy plasmon state) found to be $\sim 1 \mathrm{eV}$ and the difference between the relaxation time constants for these 2 states was $\sim 0.44$ ps $(\sim 6 \%)$.

\subsection{Conclusion}

Smith et $a l^{7}$ experimentally found a $\sim 1$ ps relaxation time constant for the $\mathrm{Au}_{55}$ clusters and a $\sim 7$ ps relaxation time constant for the $15 \mathrm{~nm}$ Au nanoparticles. The relaxation time constant for $\mathrm{Au}_{55}$ from this work is considerably larger than the experimental values reported by Smith et al. ${ }^{7}$ The attached ligands in experimental work would be one reason for this difference. Also Smith et $a l^{7}$ probed hot electrons using only one energy. So there is a possibility to miss some long living states below or above the probe energy. The following equation which includes both electron-phonon coupling and surface collision rate was used by smith et $a l^{7}$ to explain the changes of the relaxation time constant of different sizes of nanoparticles. 
$\frac{1}{\tau_{o b s}}=\frac{a R^{\alpha}}{\tau_{b u l k}}+\frac{b v_{f}}{R}$

(Equation 4.7)

where $\tau_{o b s}$ and $\tau_{\text {bulk }}$ are observed and bulk relaxation time constants, $v_{f}$ is Fermi velocity of the electrons, $R$ is radius of the nanoparticle, $\alpha$ is a positive integer, $a$ and $b$ are constants specific for the given material. Since the $2^{\text {nd }}$ term of the above equation was derived from the surface collision, if the surface collision is dominant in the relaxation process, when decreasing the size of the nanoparticle the relaxation time constant should be decreased. But longer relaxation time constant for $\mathrm{Au}_{55}(2 \mathrm{R} \sim 1.4 \mathrm{~nm})$ helps to easily rule out the surface collision dominant relaxation process for $\mathrm{Au}_{55}$. Since $1^{\text {st }}$ term of the above equation was derived from the electron-phonon coupling, if the electron-phonon coupling is dominant in the relaxation process, when decreasing the size of the nanoparticle the relaxation time constant should be increased. Probably due to the weakening of the electron-phonon coupling ${ }^{7}$ in smaller size nanoparticles. Therefore, the longer relaxation time constant of $\mathrm{Au}_{55}$ in this work can be attributed to the long living state $\mathrm{S}_{331}$ or weak electronphonon coupling or both. Also previously reported molecular like relaxation dynamics ${ }^{8}$ for $\mathrm{Au}_{55}$ can be rule out base on this results. 


\section{References}

1. A. J. Neukirch, Z. Guo and O. V. Prezhdo, The Journal of Physical Chemistry C, 2012, 116, 15034-15040.

2. Z. Guo, B. F. Habenicht, W.-Z. Liang and O. V. Prezhdo, Physical Review B, 2010, 81, 125415.

3. W. Vogel, B. Rosner and B. Tesche, The Journal of Physical Chemistry, 1993, 97, $11611-11616$.

4. J. A. Carr, H. Wang, A. Abraham, T. Gullion and J. P. Lewis, The Journal of Physical Chemistry C, 2012, 116, 25816-25823.

5. G. Schmid, W. Meyer-Zaika, R. Pugin, T. Sawitowski, J.-P. Majoral, A.-M. Caminade and C.-O. Turrin, Chemistry - A European Journal, 2000, 6, 1693-1697.

6. Y. Pei, N. Shao, Y. Gao and X. C. Zeng, ACS Nano, 2010, 4, 2009-2020.

7. B. A. Smith, J. Z. Zhang, U. Giebel and G. Schmid, Chemical Physics Letters, 1997, 270, 139-144.

8. S. H. Yau, O. Varnavski, J. D. Gilbertson, B. Chandler, G. Ramakrishna and T. Goodson, The Journal of Physical Chemistry C, 2010, 114, 15979-15985.

9. C. M. Chang, C. Cheng and C. M. Wei, The Journal of Chemical Physics, 2008, 128, 124710.

10. O. F. Sankey and D. J. Niklewski, Physical Review B, 1989, 40, 3979-3995.

11. P. Jelínek, H. Wang, J. P. Lewis, O. F. Sankey and J. Ortega, Physical Review B, 2005, 71, 235101. 
12. J. P. Lewis, P. Jelínek, J. Ortega, A. A. Demkov, D. G. Trabada, B. Haycock, H. Wang, G. Adams, J. K. Tomfohr, E. Abad, H. Wang and D. A. Drabold, physica status solidi (b), 2011, 248, 1989-2007.

13. E. Abad, J. P. Lewis, V. Zobač, P. Hapala, P. Jelínek and J. Ortega, The Journal of Chemical Physics, 2013, 138, 154106.

14. A. J. Neukirch, L. C. Shamberger, E. Abad, B. J. Haycock, H. Wang, J. Ortega, O. V. Prezhdo and J. P. Lewis, Journal of Chemical Theory and Computation, 2014, 10, 14-23.

15. C. M. Isborn, X. Li and J. C. Tully, The Journal of Chemical Physics, 2007, 126, 134307.

16. S. Meng and E. Kaxiras, The Journal of Chemical Physics, 2008, 129, 054110.

17. J. C. Tully, The Journal of Chemical Physics, 1990, 93, 1061-1071.

18. S. Hammes-Schiffer and J. C. Tully, The Journal of Chemical Physics, 1994, 101, 46574667. 


\section{Chapter 5}

\section{Plasmonic heating assisted $\mathrm{CO}_{2}$ reduction using $\underline{\text { Au-ZnO heterostructures }}$}

\subsection{Introduction}

More than $80 \%$ of the world's energy demand will be full filled by burning of fossil fuels at least for next few decades. However, in addition to energy, burning of fossil fuels releases a massive amount of $\mathrm{CO}_{2}$ to the environment. Even though energy efficient equipment and alternative energy methods help to reduce the $\mathrm{CO}_{2}$ emission, world population growth compete with it. The net result is the increasing atmospheric $\mathrm{CO}_{2}$ concentration annually and still there is no promising method to reduce the excess $\mathrm{CO}_{2}$ in the environment. Most of the artificial photocatalytic reduction of $\mathrm{CO}_{2}$ approaches is not efficient in large scale applications. The poor optical activity is one of the main problems of mostly used wide band gap metal oxide photocatalysts. The wide band gap metal oxides such as $\mathrm{TiO}_{2}$ and $\mathrm{ZnO}$ can only utilize the ultra violet region of the solar spectrum which is less than $5 \%$ of the total light coming to the earth's surface. Also, most of these photocatalysts depend on the electron-hole pair generation to drive the $\mathrm{CO}_{2}$ reduction reactions. However, the efficiency of electron-hole pair generation and their transfer in to reactants in large scale applications is very low. The oxidative corrosion ${ }^{1,2}$ is a another problem of photocatalysts which needs to be addressed with sacrificial hole scavengers. ${ }^{3}$

Remarkable optical properties of metal nanoparticles ${ }^{4}$ can be used to improve the efficiency of photocatalysis $^{5-8}$. The surface plasmon resonance of metal nanoparticles ${ }^{9,10}$ can be tuned to cover 
the entire range of visible light and extend in to near infrared range of the solar spectrum. Various plasmonic nanoparticles enhance the performance of photocatalysts under visible light excitation due to their efficient charge transfer ${ }^{11-13}$ and electric field enhancement effects. ${ }^{14,15}$ In addition to that, metal nanoparticles can generate heat under surface plasmon resonance conditions. ${ }^{4,16-18}$ In general, thermally driven catalytic processes have higher yields and are easy to implement into larger scale reactors. Therefore, it is feasible to start this in industrial settings and it is important to implement this approach to wide range of reactions utilizing this technique with high surface area heterogeneous catalysts.

It is found that there are extensive industrial applications of $\mathrm{ZnO}$ based catalysts at higher temperatures such as methanol synthesis from $\mathrm{CO}_{2}$ and water-gas shift reactions. ${ }^{19,}{ }^{20}$ Therefore, a low cost and efficient heating method for $\mathrm{ZnO}$ based catalysts will open the paths for a large number of industrial applications. In this work, the temperature of $\mathrm{ZnO}$ was elevated using the heat generated by Au nanoparticles under the surface plasmon resonance conditions. The heated $\mathrm{ZnO}$ catalytically convert the $\mathrm{CO}_{2}$ and $\mathrm{H}_{2}$ gas mixture in to $\mathrm{CH}_{4}$ and $\mathrm{CO}$.

\subsection{Light absorption and heat generation from the Au nanoparticles}

Simple model calculations were performed in order to understand the light absorption and heat generation of Au nanoparticles under different conditions. Only the absorbed light is responsible for plasmonic heating of metal nanoparticles and heating effect is very efficient and strong when the frequency of incoming electromagnetic radiation matches the surface plasmon resonance

frequency of the metal nanoparticles. ${ }^{21}$ Since the dielectric constant of metals depends on the 
frequency of the applied electric field, the amount of heat generation from Au nanoparticles also depends on the frequency of the applied electric field.

The absorption cross section was calculated according the example shown in Ohodnicki et al. ${ }^{22}$ The wavelength dependent dielectric constant of the Au which contains both standard contribution of a Drude model and the contribution from interband transitions was calculated according to Etchegoin et al. ${ }^{23}$ The optical parameters (Table 5.1) were obtained from previous reports. ${ }^{23-25}$ The following equations give absorption cross section $\left(Q_{a b s}\right)$ and wavelength dependent dielectric $\operatorname{constant}\left(\epsilon_{A u}(\lambda)\right)$ :

$Q_{a b s}=\frac{4 \pi^{2} a^{3} \sqrt{\epsilon_{m}}}{2 \lambda} \operatorname{Im}\left[\frac{\epsilon_{A u}-\epsilon_{m}}{\epsilon_{A u}+2 \epsilon_{m}}\right]$

$\epsilon_{A u}(\lambda)=\epsilon_{\infty}-\frac{1}{\lambda_{p}^{2}\left(\frac{1}{\lambda^{2}}+\frac{i}{\gamma_{p} \lambda}\right)}+\sum_{j=1,2} \frac{A_{j}}{\lambda_{j}}\left[\frac{e^{i \phi_{j}}}{\left(\frac{1}{\lambda_{j}}-\frac{1}{\lambda}-\frac{i}{\gamma_{j}}\right)}+\frac{e^{-i \phi_{j}}}{\left(\frac{1}{\lambda_{j}}+\frac{1}{\lambda}+\frac{i}{\gamma_{j}}\right)}\right]$

Where $a$ is the radius of the nanoparticle, $\epsilon_{m}$ is the dielectric constant of the matrix phase, $\lambda$ is optical wavelength, $\epsilon_{\infty}$ is the high frequency limit dielectric constant, $\lambda_{p}$ is the plasma wavelength, $\gamma_{p}$ is the damping (expressed as a wavelength), $\lambda_{j}$ is the interband transition wavelength, $\gamma_{j}$ is the transition broadening (expressed as a wavelength), $A_{j}$ is the dimensionless critical point amplitudes and $\phi_{j}$ is the phase which was fixed in to $-\pi / 4$ according to Etchegoin et al. ${ }^{23}$ 
Table 5.1 Optical parameters

\begin{tabular}{|c|c|c|}
\hline Parameter & Value & Source \\
\hline$a$ & $2.5 \mathrm{~nm}$ & Average radius from the TEM images \\
\hline$\epsilon_{m}$ (Hexane) & 1.88 & Mopsik $^{25}$ \\
\hline$\epsilon_{m}(\mathbf{Z n O})$ & 3.70 & Azad et al. ${ }^{24}$ \\
\hline $\boldsymbol{\epsilon}_{\infty}$ & 1.53 & Etchegoin et al. ${ }^{23}$ \\
\hline$\lambda_{p}$ & $145 \mathrm{~nm}$ & Etchegoin et al. ${ }^{23}$ \\
\hline$\gamma_{p}$ & $17000 \mathrm{~nm}$ & Etchegoin et al. ${ }^{23}$ \\
\hline$\lambda_{1}$ & $468 \mathrm{~nm}$ & Etchegoin et al. ${ }^{23}$ \\
\hline$\lambda_{2}$ & $331 \mathrm{~nm}$ & Etchegoin et al. ${ }^{23}$ \\
\hline$\gamma_{1}$ & $2300 \mathrm{~nm}$ & Etchegoin et al. ${ }^{23}$ \\
\hline$\gamma_{2}$ & $940 \mathrm{~nm}$ & Etchegoin et al. ${ }^{23}$ \\
\hline$A_{1}$ & 0.94 & Etchegoin et al. ${ }^{23}$ \\
\hline$A_{2}$ & 1.36 & Etchegoin et al. ${ }^{23}$ \\
\hline$\phi_{1}$ & $-\pi / 4 \mathrm{rad}$ & Etchegoin et al. ${ }^{23}$ \\
\hline$\phi_{2}$ & $-\pi / 4 \mathrm{rad}$ & Etchegoin et al. ${ }^{23}$ \\
\hline
\end{tabular}

The Real and imaginary parts of the wavelength dependent dielectric constant of the Au are shown in Figures 5.1 and 5.2. 


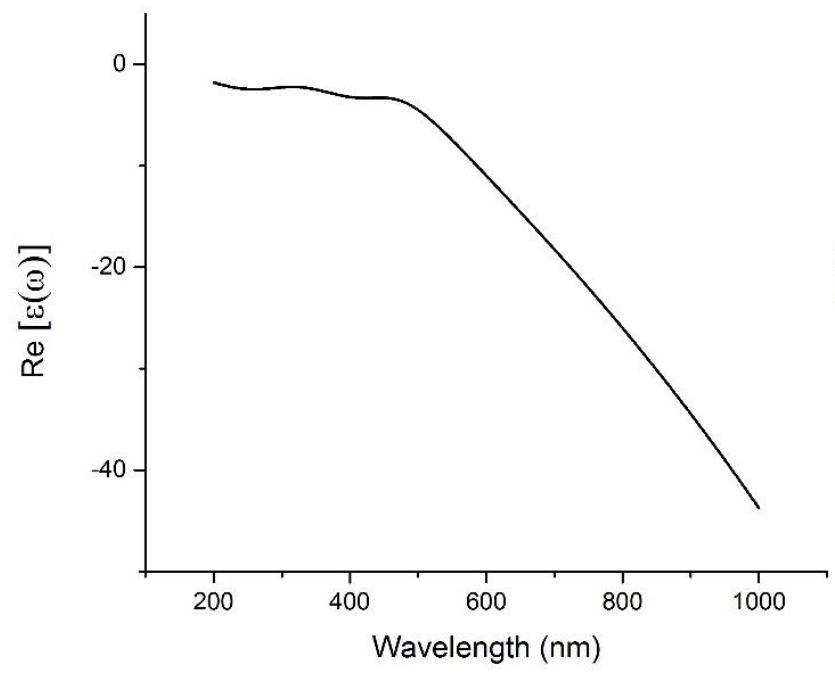

Figure 5.1 Real part of the wavelength dependent dielectric constant of $\mathrm{Au}$

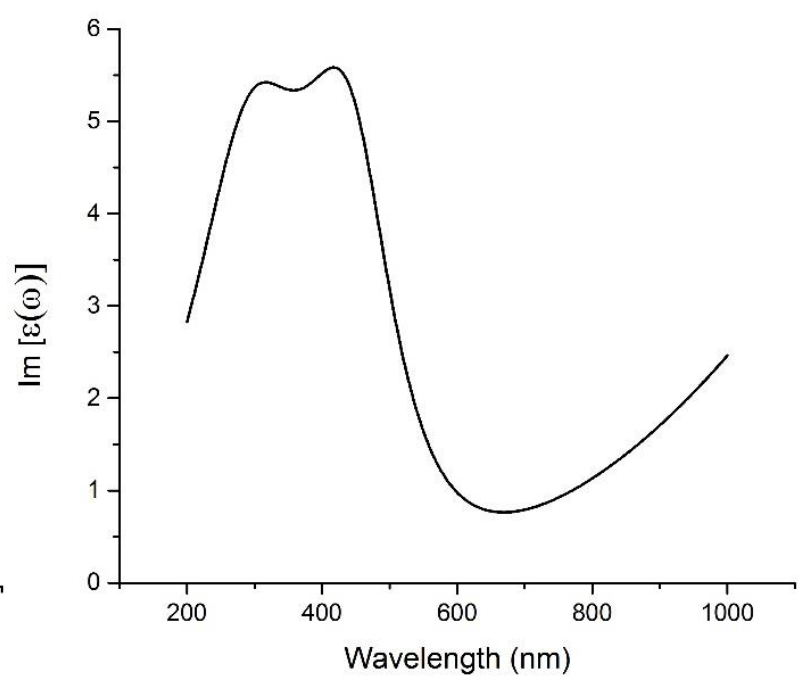

Figure 5.2 Imaginary part of the wavelength dependent dielectric constant of $\mathrm{Au}$

A simple calculation which was based on simplified Mie theory was performed according to the Govorov et $a l^{21}$ in order to calculate the temperature at surface of the spherical shape $\mathrm{Au}$ nanoparticle. The usual heat transfer equation can be used to explain the heat transfer of metal nanoparticles when there is no phase transformation. ${ }^{21}$

$\rho(r) c(r) \frac{\partial T(r, t)}{\partial t}=\nabla k(r) \nabla T(r, t)+Q(r, t)$

where $T(r, t)$ is the temperature as a function of position $r$ and time $t, \rho(r)$ is the mass density of the nanoparticle, $c(r)$ is the specific heat of nanoparticles, $k(r)$ is the thermal conductivity of nanoparticles and $Q(r, t)$ is the local heat intensity.

The local heat intensity can be described as follows ${ }^{21}$,

$Q(r, t)=<j(r, t) \cdot E(r, t)>_{t}=-\frac{1}{2} \operatorname{Re}\left[i \omega \frac{\epsilon(r)-1}{4 \pi} \tilde{E}(r) \tilde{E}^{*}(r)\right]$ 
where $j(r, t)$ is the current density, $E(r, t)=\operatorname{Re}\left[\tilde{E}(r) e^{-i \omega t}\right]$ is the induced electric field, $\epsilon(r)$ is the relative permittivity of the nanoparticles.

The external electric field which uses to excite the surface plasmon of metal nanoparticles is, $E_{0}(t)=\operatorname{Re}\left[\widetilde{E}_{0}(t) e^{-i \omega t}\right]$ and the light intensity is $I(t)=I_{0}=c E_{0}^{2} \sqrt{\epsilon_{s}} / 8 \pi$ where $c$ is the velocity of light and $\epsilon_{S}$ is the relative permittivity of the surrounding medium.

Then temperature distribution outside the spherical nanoparticle ${ }^{21}$,

$\Delta T(r)=\frac{V_{N P} Q}{4 \pi k_{s} r}\left(r>R_{N P}\right)$

where $V_{N P}$ is the volume of the nanoparticles, $R_{N P}$ is the radius of the nanoparticles and $k_{S}$ is the thermal conductivity of the surrounding medium. Then, the local heat intensity can be re-written $\operatorname{as}^{21}$,

$Q=-\operatorname{Re}\left[i \omega \frac{\varepsilon_{N P}-1}{8 \pi} E_{0}^{2}\left|\frac{3 \epsilon_{S}}{2 \epsilon_{S}+\varepsilon_{N P}}\right|^{2}\right]$

where $\varepsilon_{N P}$ is the relative permittivity of the metal nanoparticles. The maximum temperature at the surface of the nanoparticle ${ }^{21}$,

$\Delta T_{\max }\left(I_{0}\right)=\frac{R_{N P}^{2}}{3 k_{0}} \operatorname{Re}\left[i \omega \frac{1-\varepsilon_{N P}}{8 \pi}\left|\frac{3 \epsilon_{S}}{2 \epsilon_{S}+\varepsilon_{N P}}\right|^{2}\right] \frac{8 \pi I_{0}}{c \sqrt{\epsilon_{S}}}$

Figure 5.3 shows the absorption cross section of the $20 \mathrm{~nm}$ single spherical Au nanoparticle in air and in $\mathrm{ZnO}$ media as a function of illumination wavelength. The absorption maxima can be seen around $481 \mathrm{~nm}$ and $552 \mathrm{~nm}$ for Au nanoparticle in air and in $\mathrm{ZnO}$ respectively. The illumination 
intensity was $2.5^{*} 10^{5} \mathrm{~W} / \mathrm{m}^{2}$. Since the absorption cross section directly affects the heat generation one can expect the maximum heating effects to be around the maximum absorption cross section.

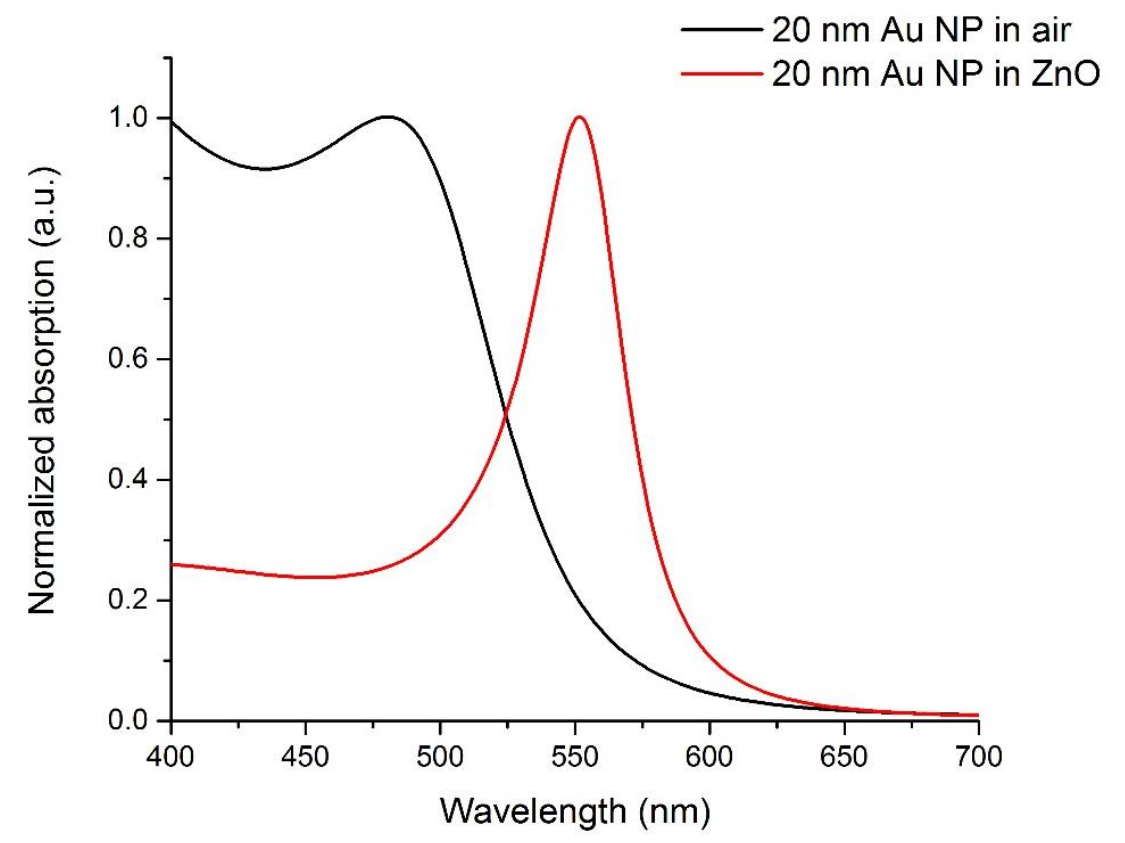

Figure 5.3 Absorption cross section of $20 \mathrm{~nm}$ spherical shaped $\mathrm{Au}$ nanoparticle in air and in $\mathrm{ZnO}$ media

Figure 5.4 shows the maximum temperature increase at the surface of the $20 \mathrm{~nm}$ spherical shaped $\mathrm{Au}$ nanoparticle embedded in $\mathrm{ZnO}$ under $2.5^{*} 10^{5} \mathrm{~W} / \mathrm{m}^{2}$ light illumination. The maximum temperature difference can be observed at $552 \mathrm{~nm}$ which also confirms that the maximum heat generation occurs at the maximum absorption. Figure 5.5 shows the temperature increase at the surface of different sizes of Au nanoparticles as function of the illumination light intensity. For a given size of Au nanoparticle, the heat generation linearly increases with the illumination light intensity. Also, according to the Figure 5.5, the heat generation increases with the size of the $\mathrm{Au}$ nanoparticle for a given illumination light intensity. However, Govorov et al ${ }^{21}$ recommended not to use this method to calculate the heat generation of Au nanoparticles which are larger than the $50 \mathrm{~nm}$ since they used a simplified Mie theory to derive the above equations. The heat generation 
can be strongly enhanced in the presence of more than one Au nanoparticle due to the cumulative effect and Coulomb interactions. ${ }^{26}$

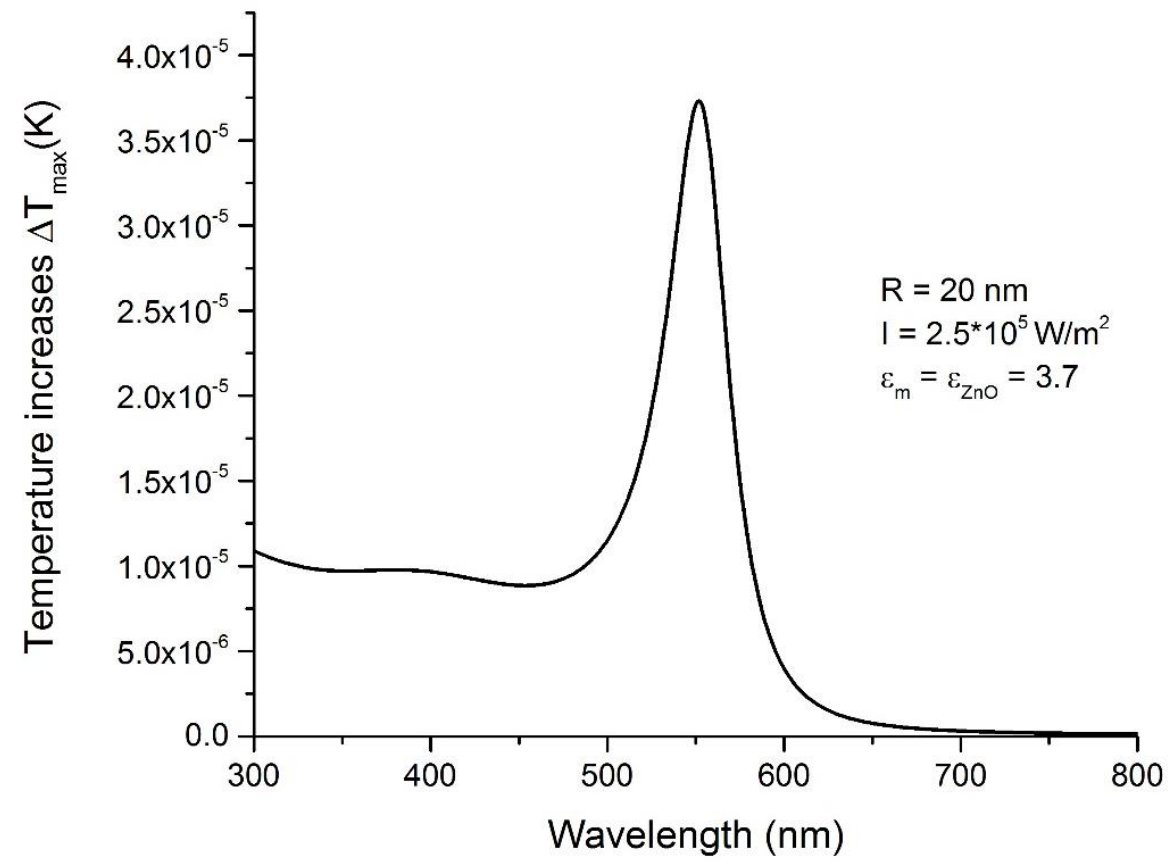

Figure 5.4 Temperature increase of $20 \mathrm{~nm}$ spherical shaped Au nanoparticle embedded in $\mathrm{ZnO}$

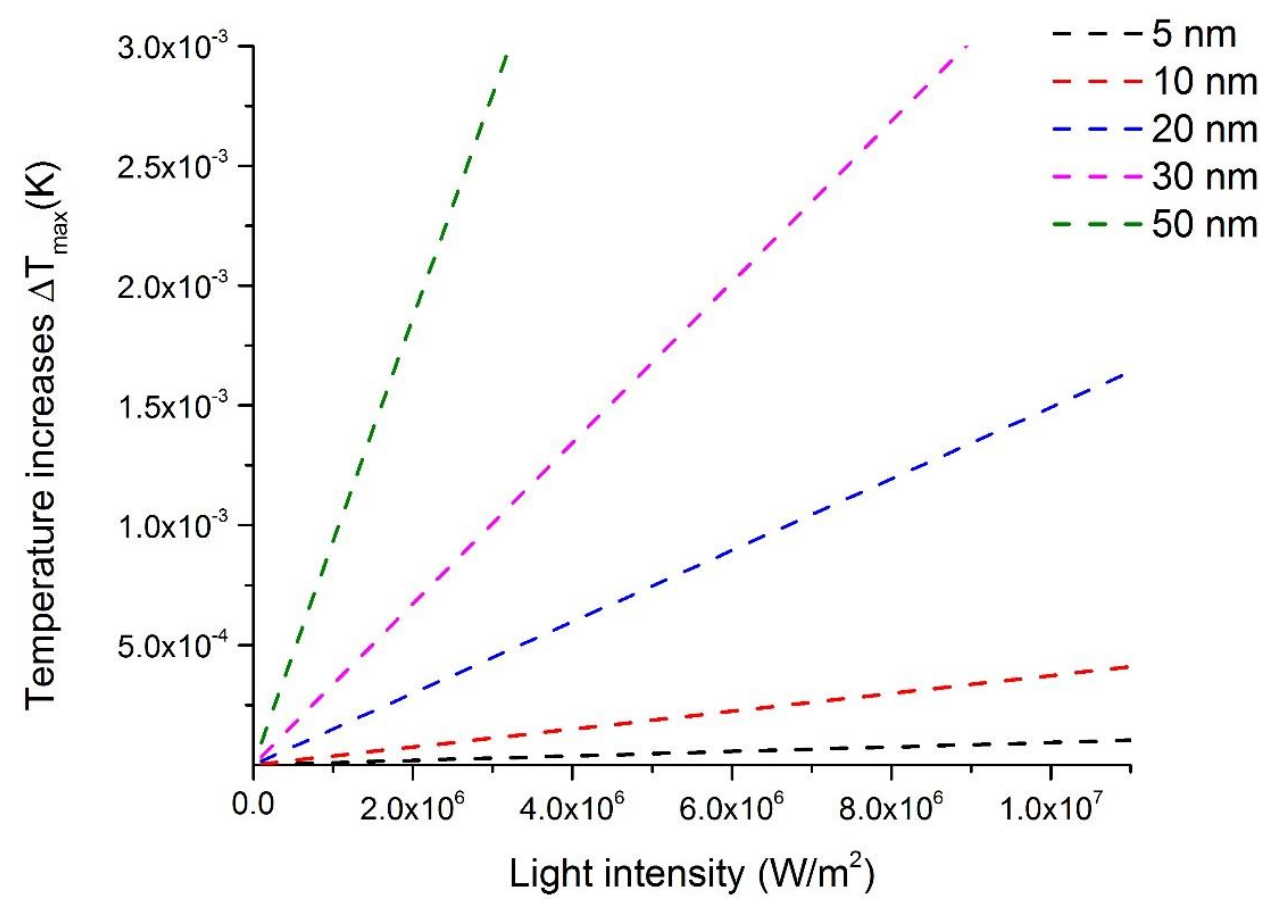

Figure 5.5 Temperature increase of different sizes of spherical shaped Au nanoparticles embedded in $\mathrm{ZnO}$ 
Based on the above model calculations; the surrounding medium, size of the Au nanoparticles and the illumination light intensity play critical roles in plasmonic heat generation. In order to achieve the maximum light-to-heat conversion efficiency, it is necessary to have a narrow plasmon resonance peak which closely matches the illumination light frequency or a broadband light source that matches the bandwidth of the resonance. A single Au nanoparticle which is fully embedded in $\mathrm{ZnO}$ was considered for the above model calculations. Also, it was assumed that there are no other Au nanoparticles in the vicinity (assumed that there is no accumulative effects and coulomb interactions). However, if the Au nanoparticles are agglomerated (clustering) heat generation might be increased by several orders of magnitude. Govorov et al ${ }^{21}$ showed the 5 fold increase of heat generation in a $4 * 4$ array (total of 16 nanoparticles) of Au nanoparticles compared to the single Au nanoparticle. This allows us to hypothesize that the local temperature of $\mathrm{ZnO}$ can be increased by a couple of hundreds of degrees, via the simultaneously illumination of clusters which contain a large number of Au nanoparticles.

Therefore, the first objective in this work was to synthesize $\mathrm{Au}-\mathrm{ZnO}$ heterostructures with moderate size Au nanoparticles which show a strong and narrow absorption peak close to $532 \mathrm{~nm}$ (illumination wavelength). The simple wet chemical method was implemented in order to achieve this objective is discussed in detail in Section 5.3.

\subsection{Materials preparation}

Hydrogen tetrachloroaurate(III) hydrate $\left(\mathrm{HAuCl}_{4} \cdot \mathrm{xH}_{2} \mathrm{O}\right), 99.9 \%$, was purchased from Alfa Aesar. $\mathrm{ZnO}$ nanoparticles and $\mathrm{SiO}_{2}(99.995+\%)$ were obtained from Meliorum Technologies and Sigma-Aldrich, respectively. 


\subsubsection{Synthesis of Au-ZnO heterostructured catalysts}

$\mathrm{ZnO}$ nanoparticles $(0.3 \mathrm{~g})$ were dispersed in a small amount of methanol to form a paste. Then, HAucl $\mathrm{xH}_{2} \mathrm{O}$ (according to Table 5.1) was dissolved in a small amount of methanol and was slowly added to the $\mathrm{ZnO}$ paste under vigorous stirring to form a uniform mixture. The uniform mixture was dried under a small $\mathrm{N}_{2}$ flow until methanol was evaporated. The dried sample was heat treated at the annealing temperature $\left(100{ }^{\circ} \mathrm{C}-800{ }^{\circ} \mathrm{C}\right)$ for 1 hour in air. The temperature was increased from room temperature to annealing temperature with the rate of $10{ }^{\circ} \mathrm{C} \mathrm{min}^{-1}$. After cooling the sample to the room temperature, a pink-red colored powder was observed. These Au$\mathrm{ZnO}$ powder samples were used for characterizations, Raman spectral analysis and $\mathrm{CO}_{2}$ reduction reactions, without further processing. Ten different samples (Table 5.2) were synthesized according to the above procedure at different annealing temperatures and Au concentrations. Au$\mathrm{SiO}_{2}$ was synthesized using the same method as $\mathrm{Au}-\mathrm{ZnO}$.

Table 5.2 Au-ZnO samples synthesized at different annealing temperatures and different Au concentrations

\begin{tabular}{|c|c|c|}
\hline Sample \# & Annealing temperature $\left({ }^{\circ} \mathbf{C}\right)$ & Au concentration by weight $\%$ \\
\hline 1 & 100 & 5 \\
\hline 2 & 200 & 5 \\
\hline 3 & 200 & 10 \\
\hline 4 & 200 & 5 \\
\hline 5 & 300 & 10 \\
\hline 6 & 300 & 15 \\
\hline 7 & 300 & 5 \\
\hline 8 & 400 & 5 \\
\hline 9 & 600 & 5 \\
\hline 10 & 800 & \\
\hline
\end{tabular}




\subsection{Characterization of the $\mathrm{Au}-\mathrm{ZnO}$ heterostructures}

\subsubsection{Diffuse reflectance spectroscopy characterization}

The optical properties of the Au- $\mathrm{ZnO}$ and $\mathrm{ZnO}$ were characterized using the Perkin Elmer Lambda $1050 \mathrm{UV} / \mathrm{Vis} / \mathrm{NIR}$ spectrometer equipped with an integrating sphere. Right after the synthesis diffuse reflectance spectra were taken for all the samples. Au-ZnO samples which were annealed at $200{ }^{\circ} \mathrm{C}$ and $300{ }^{\circ} \mathrm{C}$ showed very narrow absorption peaks compared to the other samples close to the $532 \mathrm{~nm}$ wavelength (Figure 5.6). The broadening of the absorption peak was observed when increasing the annealing temperature due to the large size distribution. All the samples showed an absorption peak around $532 \mathrm{~nm}$ due to the surface plasmon resonance of Au nanoparticles. However, the above mentioned two samples can be identified as the best $\mathrm{Au}-\mathrm{ZnO}$ candidates to excite the surface plasmon resonance of Au nanoparticles using the $532 \mathrm{~nm}$ green laser. Among the three different Au concentrations (5\%,10\% and 15\%) which were annealed at $200{ }^{\circ} \mathrm{C}$ and 300 ${ }^{\circ} \mathrm{C}, 5 \% \mathrm{Au}-\mathrm{ZnO}$ samples (for both annealing temperatures) showed a very strong absorption peak close to $532 \mathrm{~nm}$ wavelength (Figure 5.7) most probably due to the narrow size distribution. The higher concentration of Au may increase the size distribution of the Au nanoparticles.

According to the model heat generation calculations the maximum heat generation can be achieved at the maximum light absorption. Since 5\% Au- $\mathrm{ZnO}$ heterostructure sample which annealed at the $300{ }^{\circ} \mathrm{C}$ shows the strong and narrow light absorption around $\sim 538 \mathrm{~nm}$ due to the surface plasmon resonance of Au nanoparticles, it was the best $\mathrm{Au}-\mathrm{ZnO}$ candidate for the maximum heat generation under the $532 \mathrm{~nm}$ light illumination. 


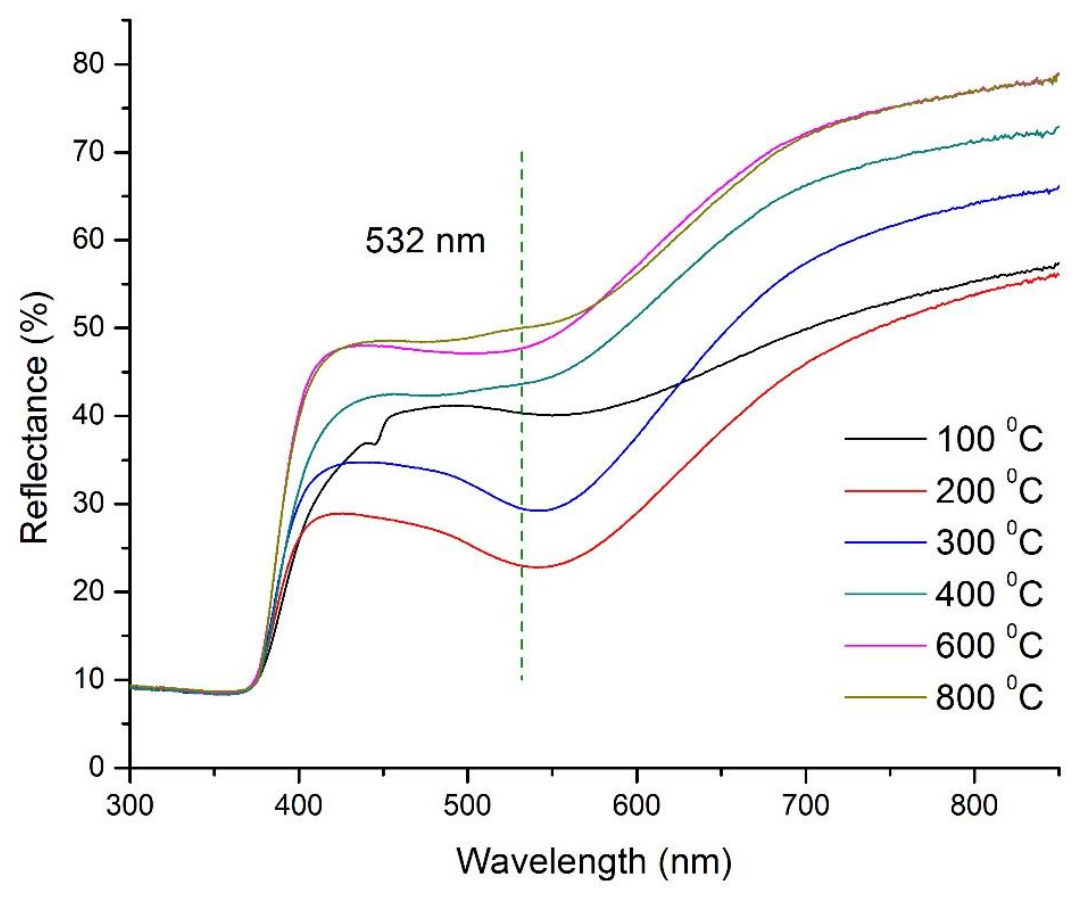

Figure 5.6 Diffuse Reflectance spectra for 5\% Au-ZnO heterostructure samples at different annealing temperatures

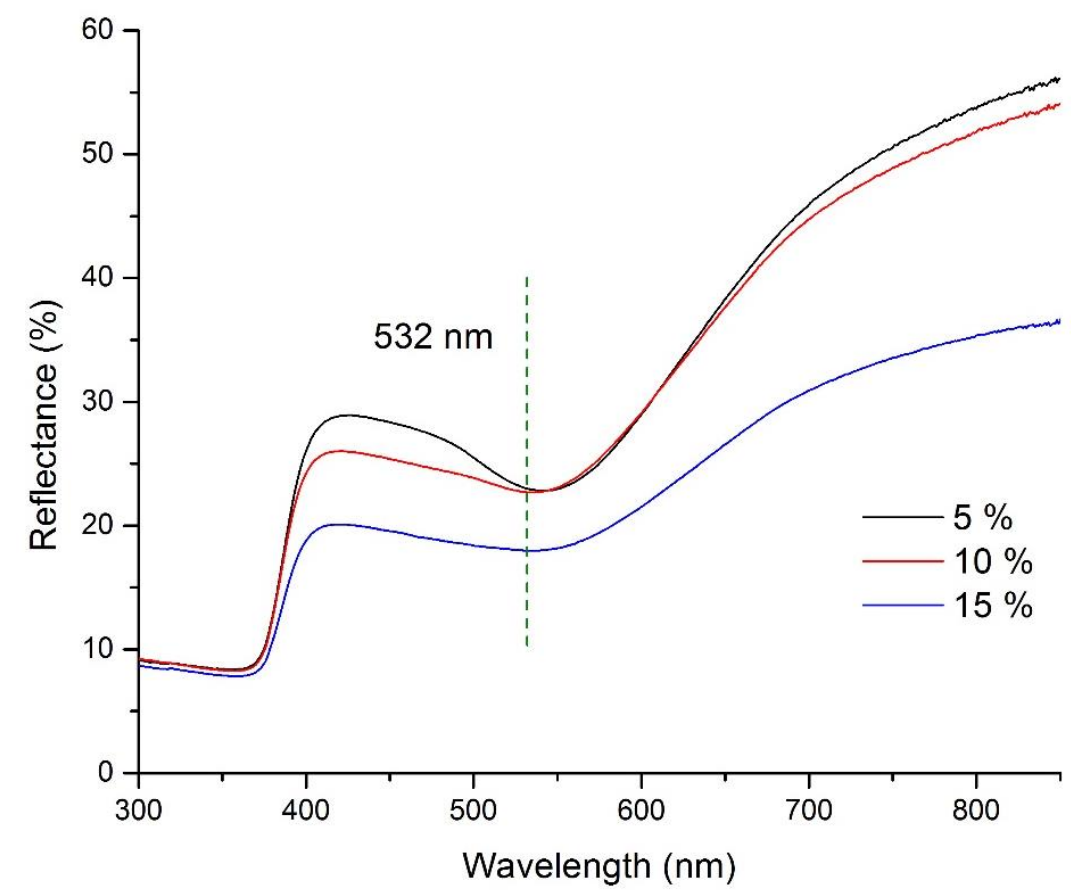

Figure 5.7 Diffuse Reflectance spectra for $\mathrm{Au}-\mathrm{ZnO}$ heterostructure samples with different $\mathrm{Au}$ concentration annealed at $200{ }^{\circ} \mathrm{C}$ 


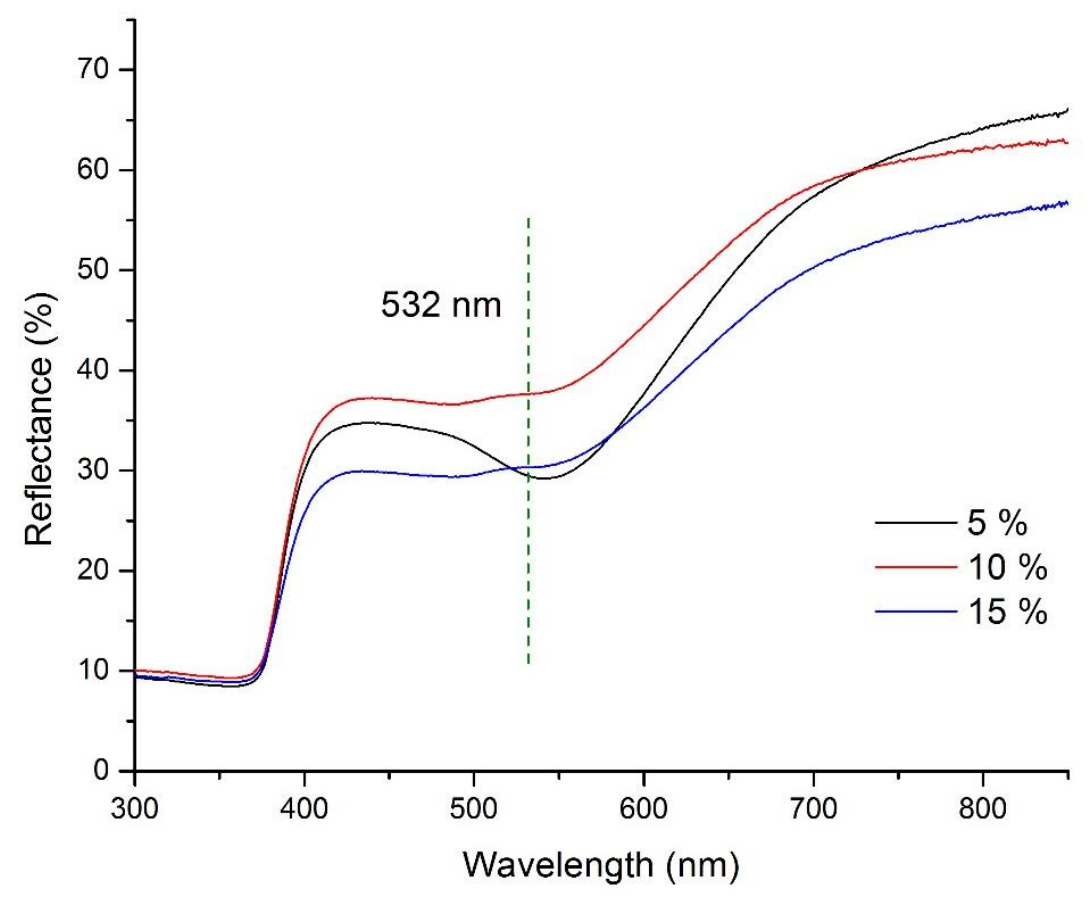

Figure 5.7 Diffuse Reflectance spectra for $\mathrm{Au}-\mathrm{ZnO}$ heterostructure samples with different Au concentration annealed at $300{ }^{\circ} \mathrm{C}$

It was expected the convenient resonance of the surface plasmon of Au nanoparticles with $532 \mathrm{~nm}$ green lasers which were utilized for the experiments in this work. This can be confirmed by the comparison of Diffuse Reflectance spectra of pure $\mathrm{ZnO}$ and 5\% Au- $\mathrm{ZnO}$ (Figure 5.8). According to the Figure 5.8, it can be clearly seen that the $532 \mathrm{~nm}$ green light absorption only happens on $\mathrm{Au}-\mathrm{ZnO}$ heterostructures. Also the surface plasmon resonance peak around $\sim 538 \mathrm{~nm}$ is consistent with the calculated absorption spectra of $20 \mathrm{~nm}$ Au nanoparticle embedded in $\mathrm{ZnO}$ which shows the absorption maximum at $552 \mathrm{~nm}$. The Au nanoparticles in 5\% Au- $\mathrm{ZnO}$ sample were around $20 \mathrm{~nm}$ (see the section 5.3.4) but they were not fully embedded in $\mathrm{ZnO}$. Since the surrounding dielectric medium directly affects the absorption cross section, it is acceptable to see a small difference between the calculated and experimental values. The strong absorption around $\sim 380$ $\mathrm{nm}$ can be attributed to the band edge absorption of the $\mathrm{ZnO}$. 


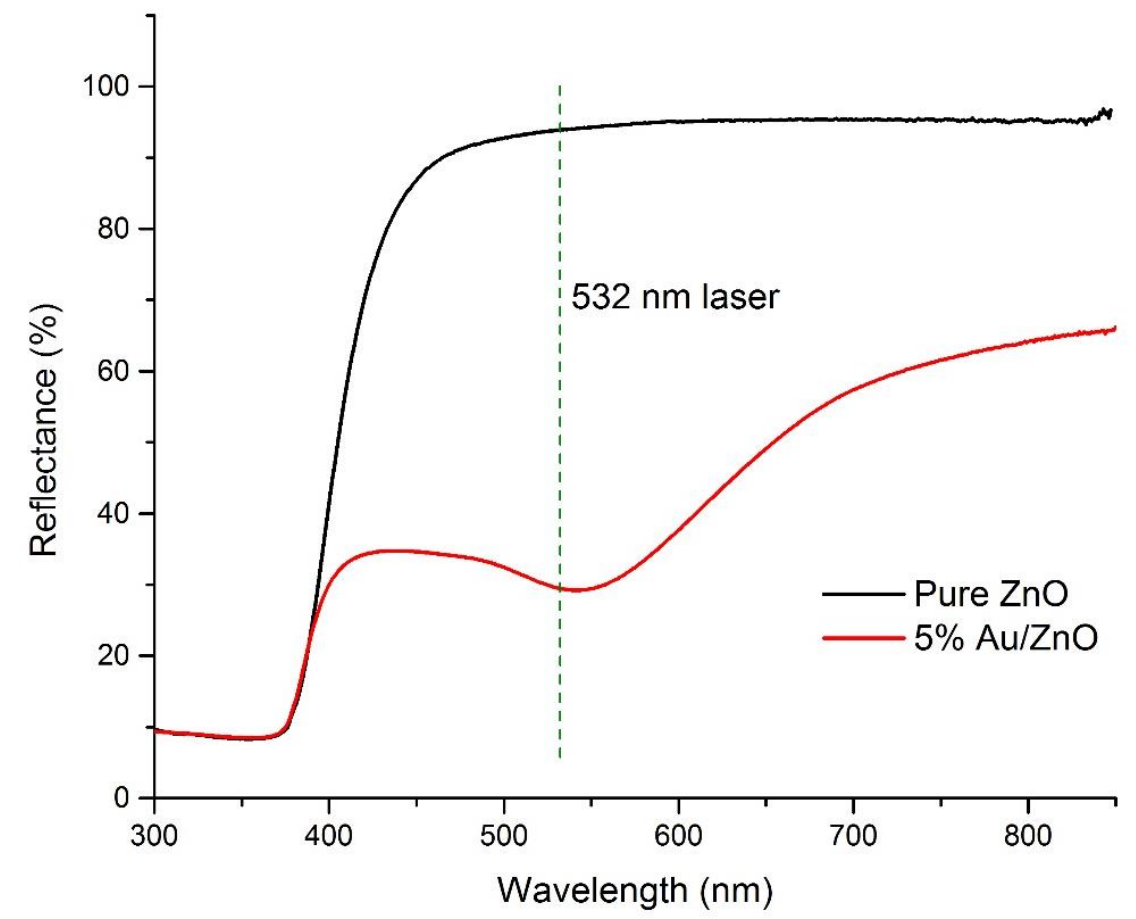

Figure 5.8 Comparison of Diffuse Reflectance spectra for pure $\mathrm{ZnO}$ and $5 \% \mathrm{Au}-\mathrm{ZnO}$

The reproducibility of the catalyst is very important in large scale industrial applications. Therefore, three batches of $5 \% \mathrm{Au}-\mathrm{ZnO}$ heterostructure samples were synthesized in order to confirm the reproducibility. The Diffuse Reflectance spectra were well consistent with each other with the same absorption maximum around $\sim 538 \mathrm{~nm}$ (Figure 5.9). 


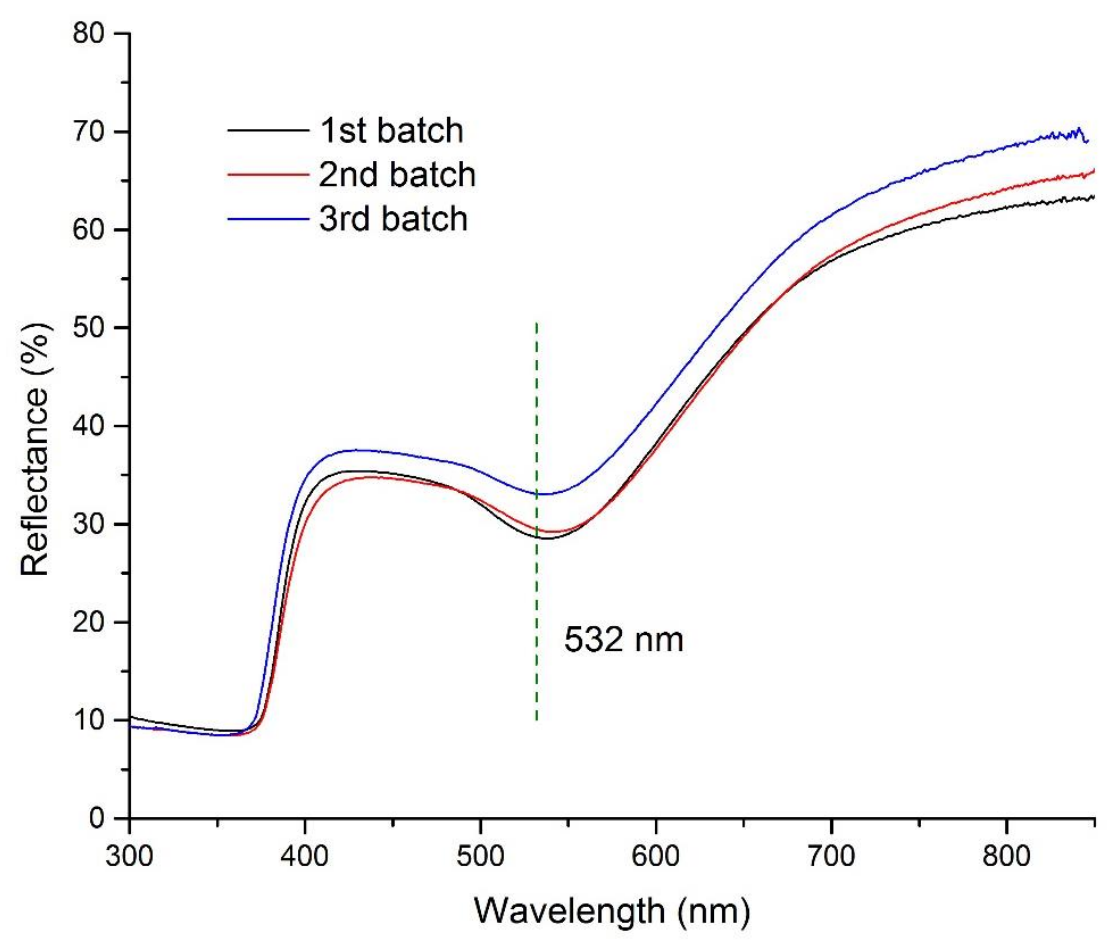

Figure 5.9 Comparison of Diffuse Reflectance spectra for 3 different batches of $5 \% \mathrm{Au}-\mathrm{ZnO}$

\subsubsection{Scanning Electron Microscopy characterization}

JEOL JSM-7600F Scanning Electron Microscope was used to investigate the surface morphology of the $\mathrm{Au}-\mathrm{ZnO}$ samples. Back scattered detector (COMPO2) at $15 \mathrm{kV}$ was used to enhance the contrast of the Au nanoparticles, relative to the contrast of $\mathrm{ZnO}$ nanoparticles. Secondary electron detector (SEI) gives better images than back scattered detector but due to the similarity of shape and order of the particle size it is hard to see the difference between $\mathrm{Au}$ and $\mathrm{ZnO}$ (Figure 5.10). The bright white dots represent the Au nanoparticles in SEM images which were obtained with back scattered detector. 

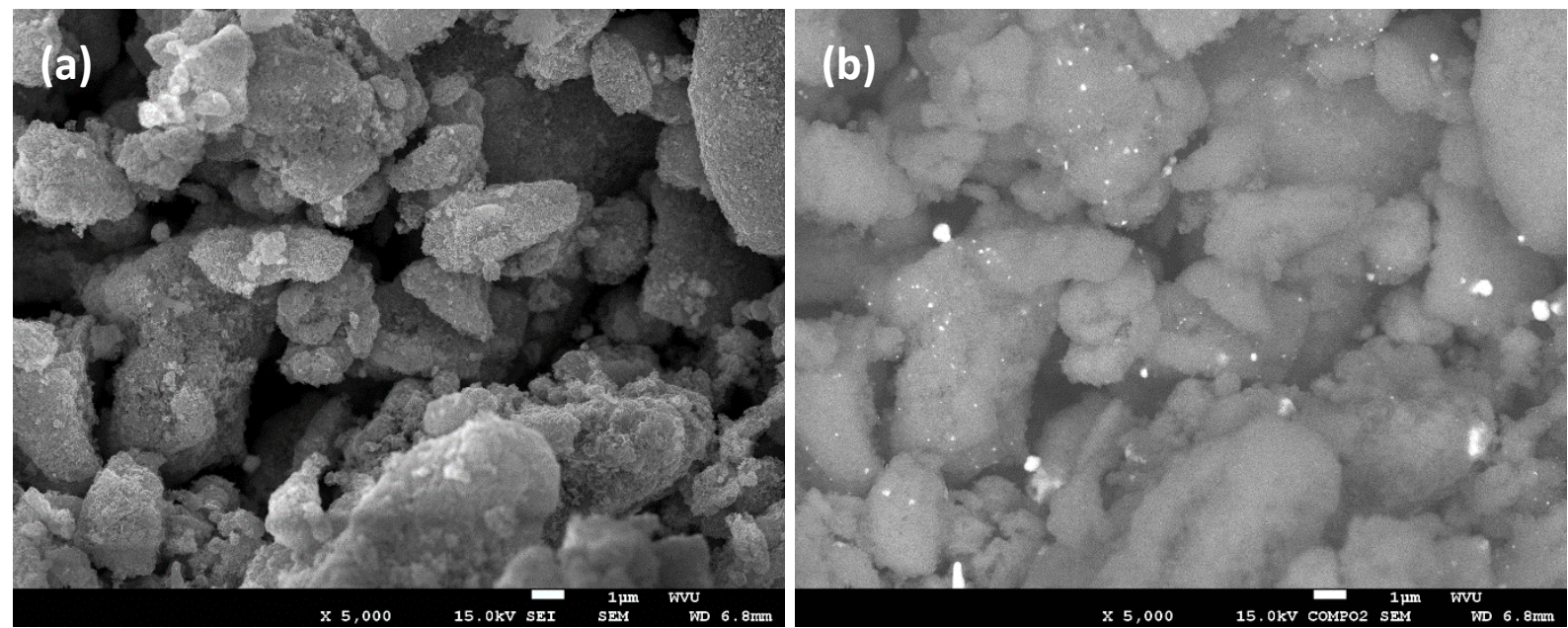

Figure 5.10 Comparison of SEM images at the same position of Au- $\mathrm{ZnO}$ sample using two different detectors (a) SEI and (b) COMPO2

According to the Figures 5.11 to 5.16, an uneven distribution of Au nanoparticles in all $\mathrm{Au}-\mathrm{ZnO}$ samples can be clearly observed. Au nanoparticles are decorating the $\mathrm{ZnO}$ surface at random positions. Figure 5.17 shows SEM images of four different positons of the 5\% Au-ZnO which was annealed at the $300{ }^{0} \mathrm{C}$. This is an evidence to confirm the arbitrary locations of the $\mathrm{Au}$ nanoparticles in $\mathrm{Au}-\mathrm{ZnO}$ samples. However, at least a few Au nanoparticles can be found when consider the $13 \mu \mathrm{m}$ experimental laser spot size which has used for the Raman spectral analysis and catalytic reactions. Also, the larger Au clusters were observed for all the $\mathrm{Au}-\mathrm{ZnO}$ samples. It is difficult to make any accurate measurement of the size of the Au nanoparticles because of the clustering of the particles. But $\mathrm{Au}-\mathrm{ZnO}$ samples which were annealed at $400{ }^{0} \mathrm{C}, 600{ }^{0} \mathrm{C}$ and 800 ${ }^{0} \mathrm{C}$ show a larger particle size distribution compared to the $\mathrm{Au}-\mathrm{ZnO}$ sample which was annealed at $300{ }^{\circ} \mathrm{C}$. This larger size distribution is consistent with the broadening of the Diffuse Reflectance spectra for the $\mathrm{Au}-\mathrm{ZnO}$ samples which were annealed at higher temperatures. Only $5 \% \mathrm{Au}-\mathrm{ZnO}$ which was annealed at $300{ }^{\circ} \mathrm{C}$ used for the rest of the experiments. 


\subsubsection{BET surface area measurements}

Surface areas of the catalysts were determined from $\mathrm{N}_{2}$ adsorption isotherms at $77 \mathrm{~K}$ using a Quantachrome Autosorb 1-C. Multipoint BET measurements were performed at relative pressures $(P / P 0)$ in the range of $0.1-0.3$. Prior to the measurements, samples were degassed under vacuum at $110{ }^{\circ} \mathrm{C}$ for $4 \mathrm{~h}$. The BET surface areas of $\mathrm{ZnO}$ (Meliorum) (after calcination at $300{ }^{\circ} \mathrm{C}$ for $1 \mathrm{~h}$ in air) and $5 \% \mathrm{Au}-\mathrm{ZnO}$ catalyst are 10 and $12 \mathrm{~m}^{2} \mathrm{~g}^{-1}$ respectively.

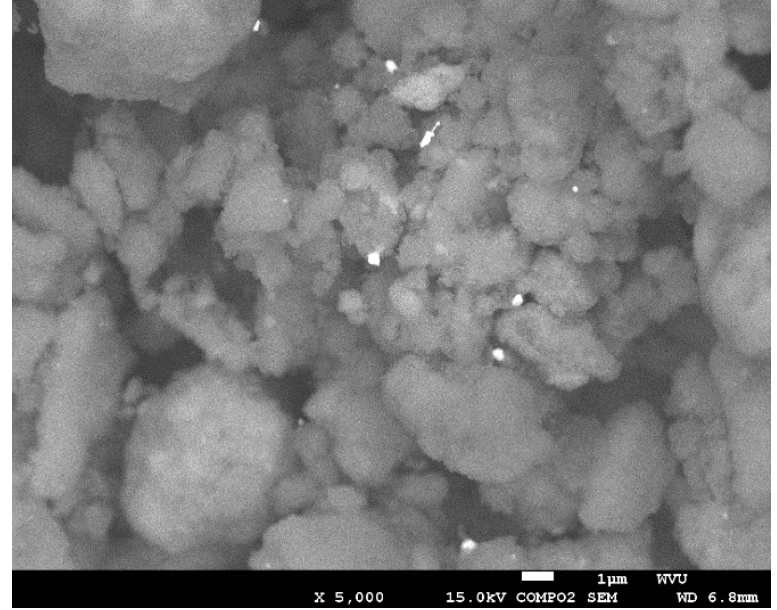

Figure 5.11 5\% Au-ZnO annealed at $100{ }^{\circ} \mathrm{C}$

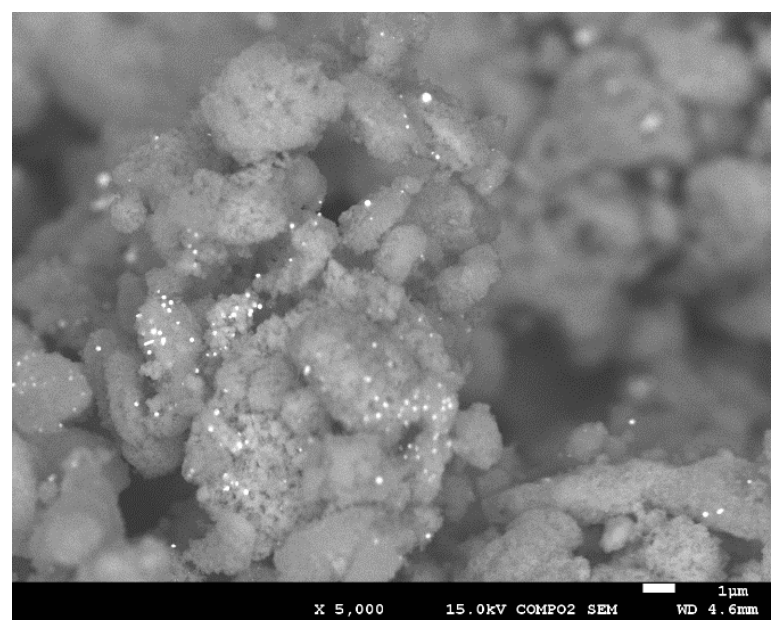

Figure 5.13 5\% Au- $\mathrm{ZnO}$ annealed at $300{ }^{\circ} \mathrm{C}$

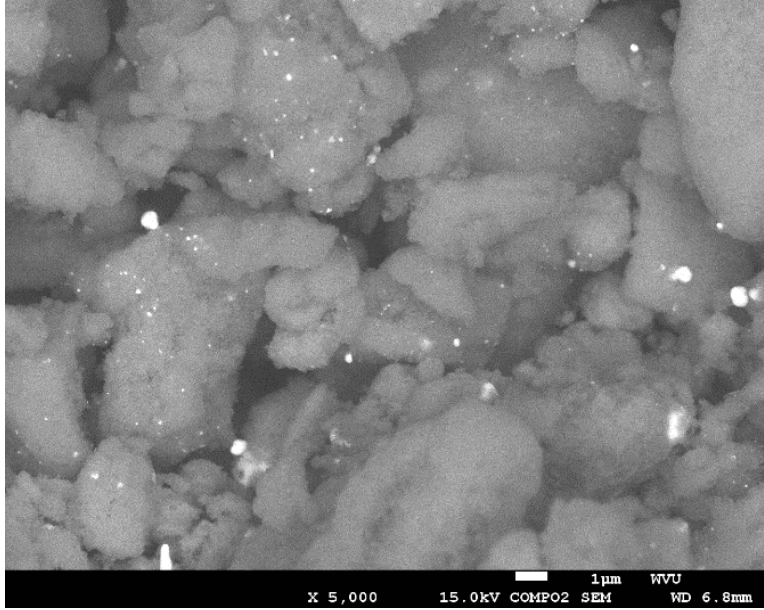

Figure 5.12 5\% Au- $\mathrm{ZnO}$ annealed at $200{ }^{\circ} \mathrm{C}$

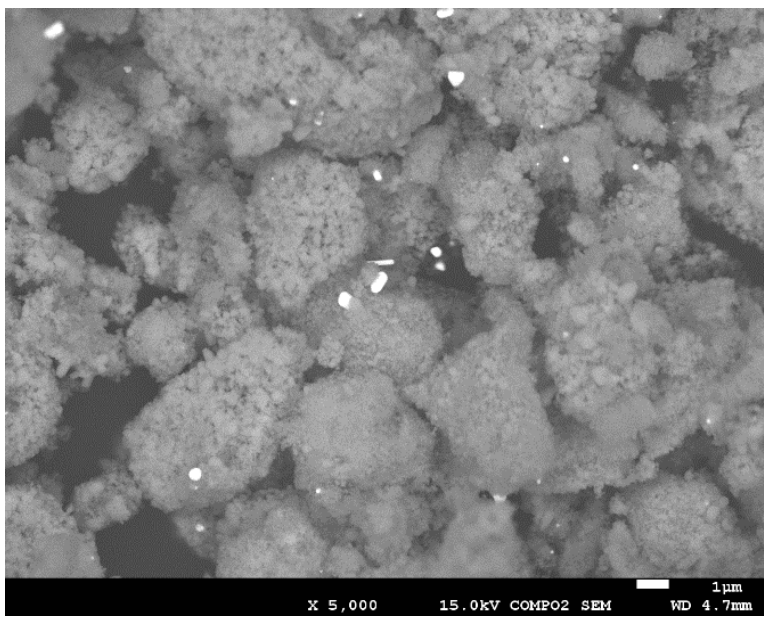

Figure 5.14 5\% Au- $\mathrm{ZnO}$ annealed at $400{ }^{\circ} \mathrm{C}$ 


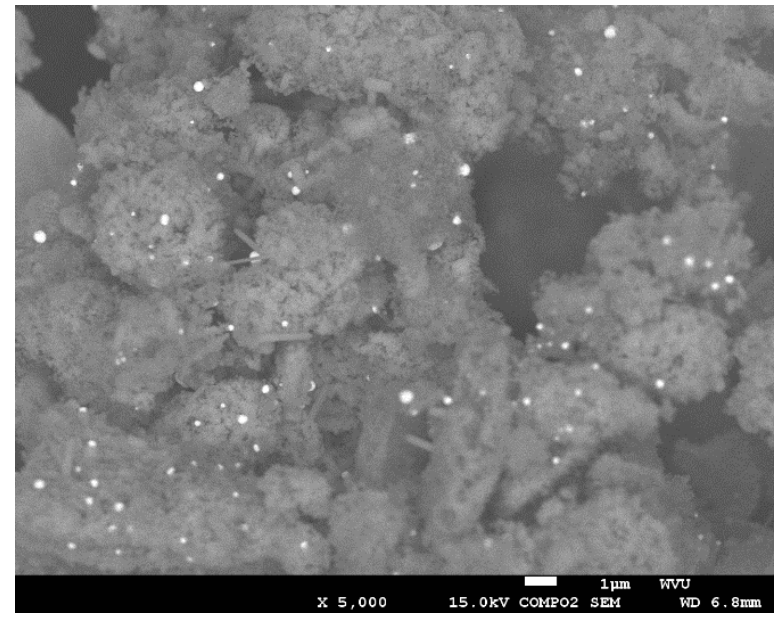

Figure 5.15 5\% Au-ZnO annealed at $600{ }^{\circ} \mathrm{C}$

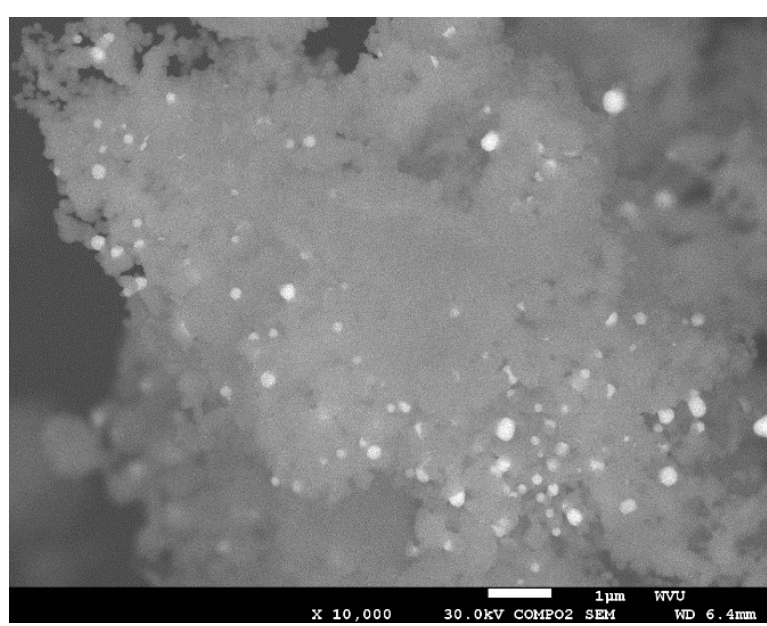

Figure 5.16 5\% Au- $\mathrm{ZnO}$ annealed at $800{ }^{\circ} \mathrm{C}$
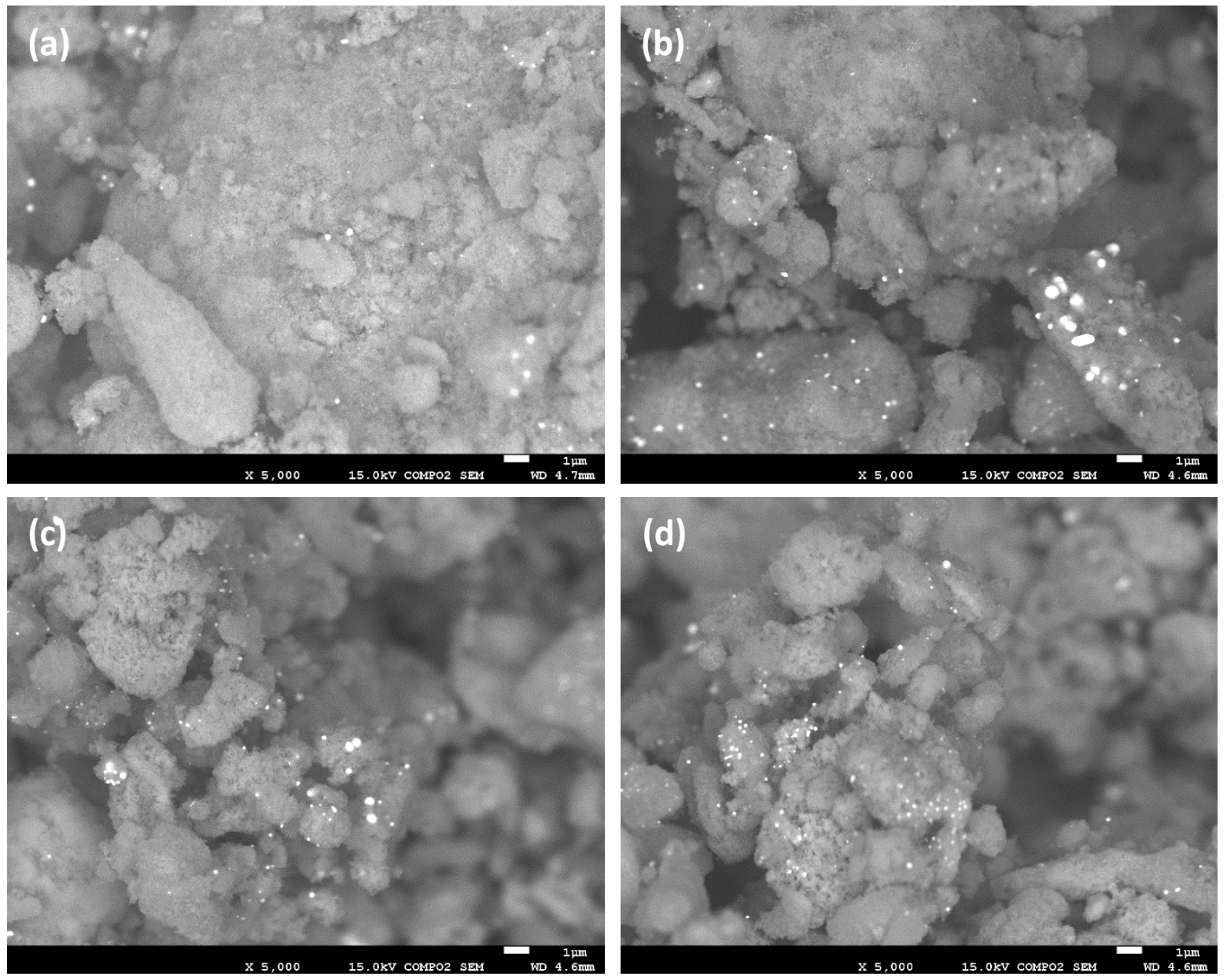

Figure 5.17 Different positions of 5\% $\mathrm{Au}-\mathrm{ZnO}$ sample 


\subsubsection{Transmission Electron Microscopy characterization}

JEOL JEM-2100 Transmission Electron Microscope was used to take both conventional and High Resolution Transmission Electron Microscope images. Instrument was operated at $200 \mathrm{kV}$. Conventional images were obtained using the Gatan ES500W digital camera which is capable of high speed and high quality imaging with a field of view larger than the traditional TEM film. High resolution images were obtained using the Orius SC1000, which is the latest generation of large format (11 Megapixel) retractable and fiber-optical coupled CCD cameras. Figure 5.18 shows the TEM image of 5\% Au-ZnO and Figure 5.19 shows the HRTEM image of the 5\% Au$\mathrm{ZnO}$. The average size of the Au nanoparticle was found to be $\sim 20 \mathrm{~nm}$.

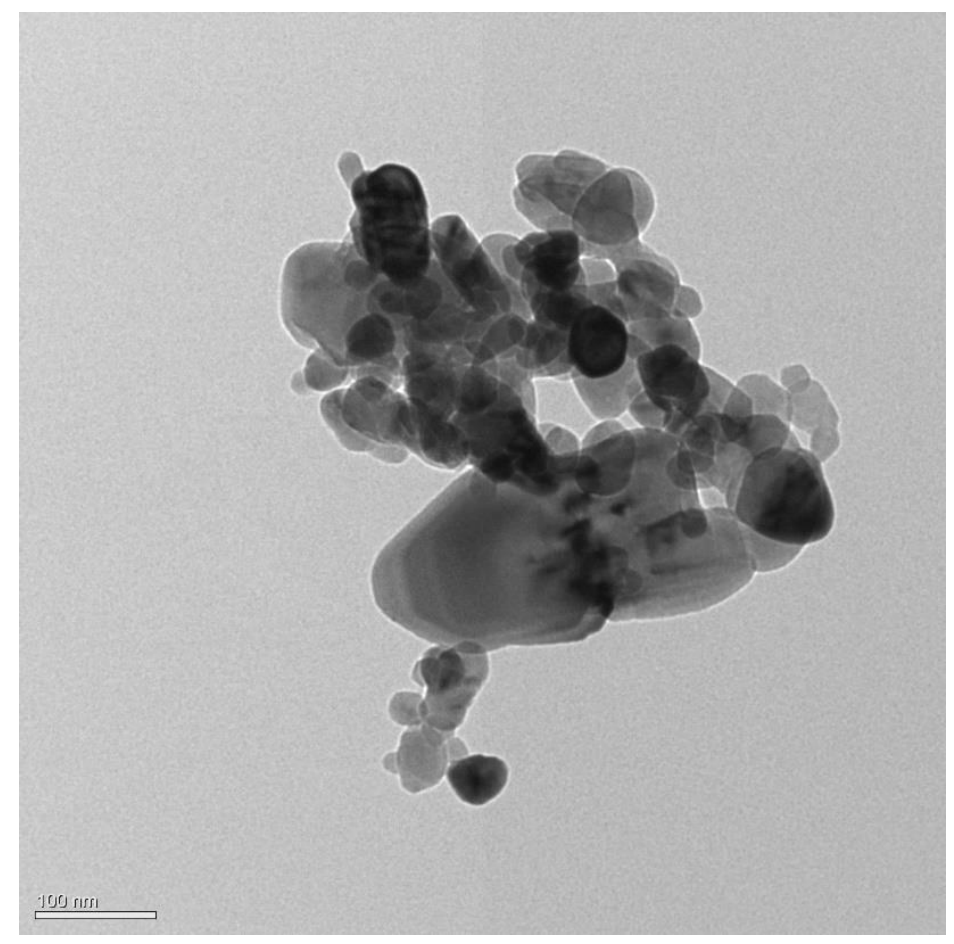

Figure 5.18 TEM image of 5\% Au-ZnO sample 


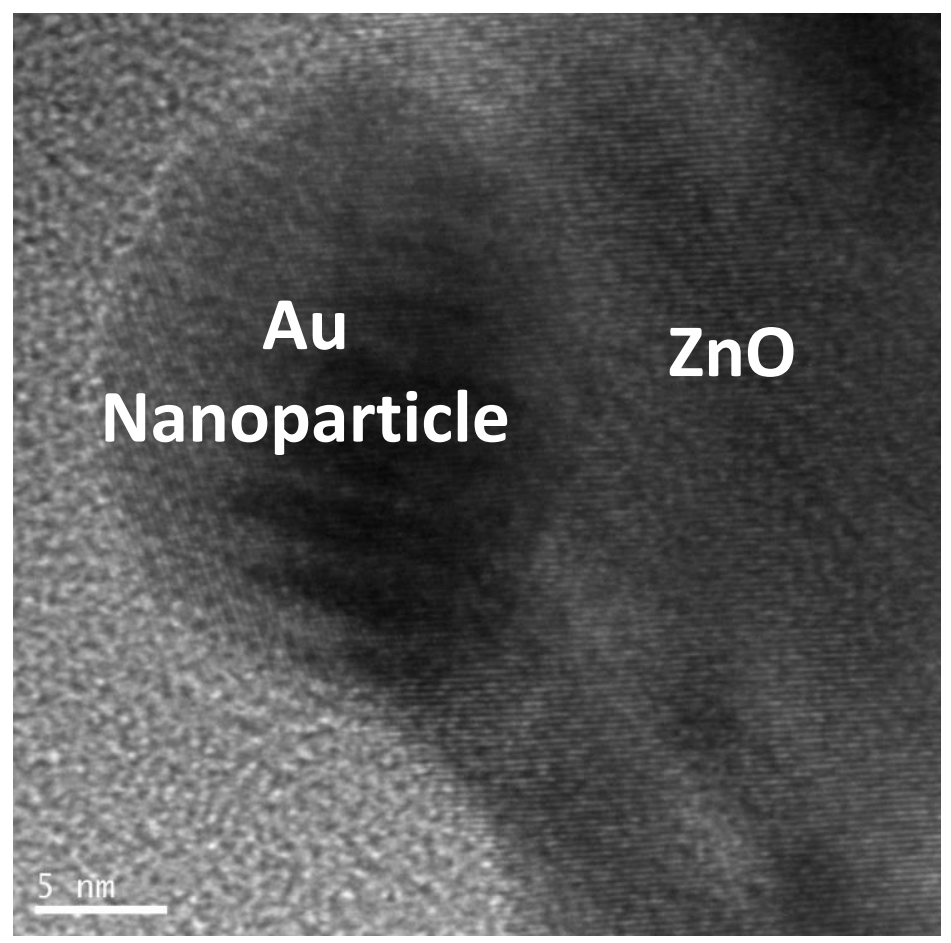

Figure 5.19 HRTEM image of 5\% Au-ZnO sample

\subsubsection{X-Ray Diffraction patterns characterization}

PANalytical X'pert Pro powder X-Ray diffractrometer was used to investigate the crystal structure information of $\mathrm{Au}-\mathrm{ZnO}$ heterostructures and instrument was operated at $40 \mathrm{kV}$ and $40 \mathrm{~mA}$. According to the X-Ray diffraction patterns, Au nanoparticles showed cubic structure and $\mathrm{ZnO}$ nanoparticles showed hexagonal wurtzite structure with high crystallinity (Figure 5.20). 


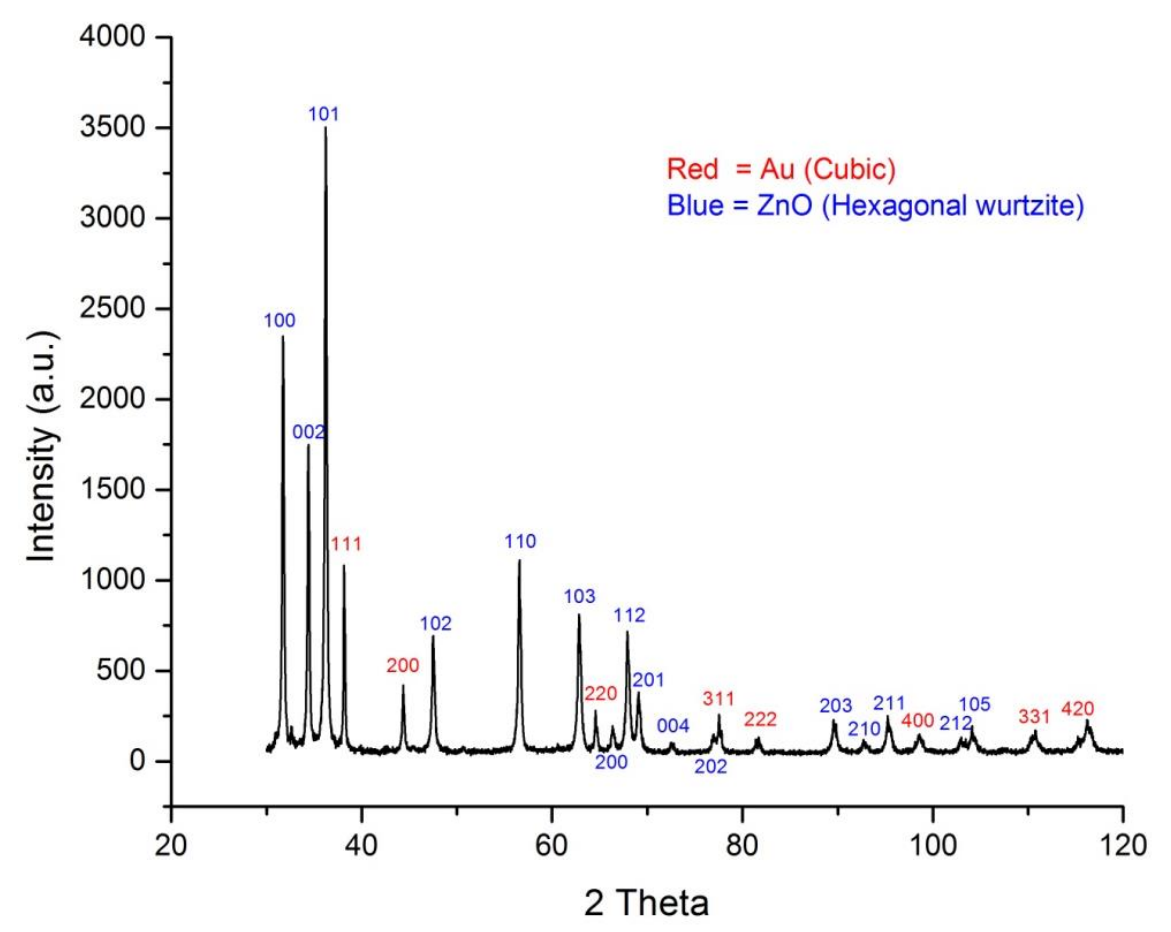

Figure 5.20 X-Ray Diffraction patterns of 5\% Au-ZnO

\subsection{Local temperature estimation studies of the $\mathrm{Au}-\mathrm{ZnO}$ heterostructures}

$\mathrm{ZnO}$ is a well-known catalyst for $\mathrm{CO}_{2}$ reduction at higher temperatures. Therefore $\mathrm{ZnO}$ is a good candidate for plasmonic heating assisted catalysis. It is very challenging to measure the temperature increases on $\mathrm{ZnO}$ due to the heat generation by the $\mathrm{Au}$ nanoparticles under surface plasmon resonance. Since heat generation of $\mathrm{Au}$ nanoparticles is a highly localized process in nanoscale. Bulk instruments cannot directly measure the local temperature of $\mathrm{ZnO}$. However, it is very important to know the local temperature of $\mathrm{ZnO}$ for the industrial catalysis applications. In this work, temperature dependent phonon spectrum of $\mathrm{ZnO}$ was used to estimate the local temperature of $\mathrm{ZnO}$. 


\subsubsection{Optical phonon modes of $\mathrm{ZnO}$}

The hexagonal wurtzite and zincblende are the two main structures of $\mathrm{ZnO}$. However, hexagonal wurtzite structure (Figure 5.21) is the most stable and the common one. The melting point is $1975{ }^{0} \mathrm{C}$ for bulk $\mathrm{ZnO}$.

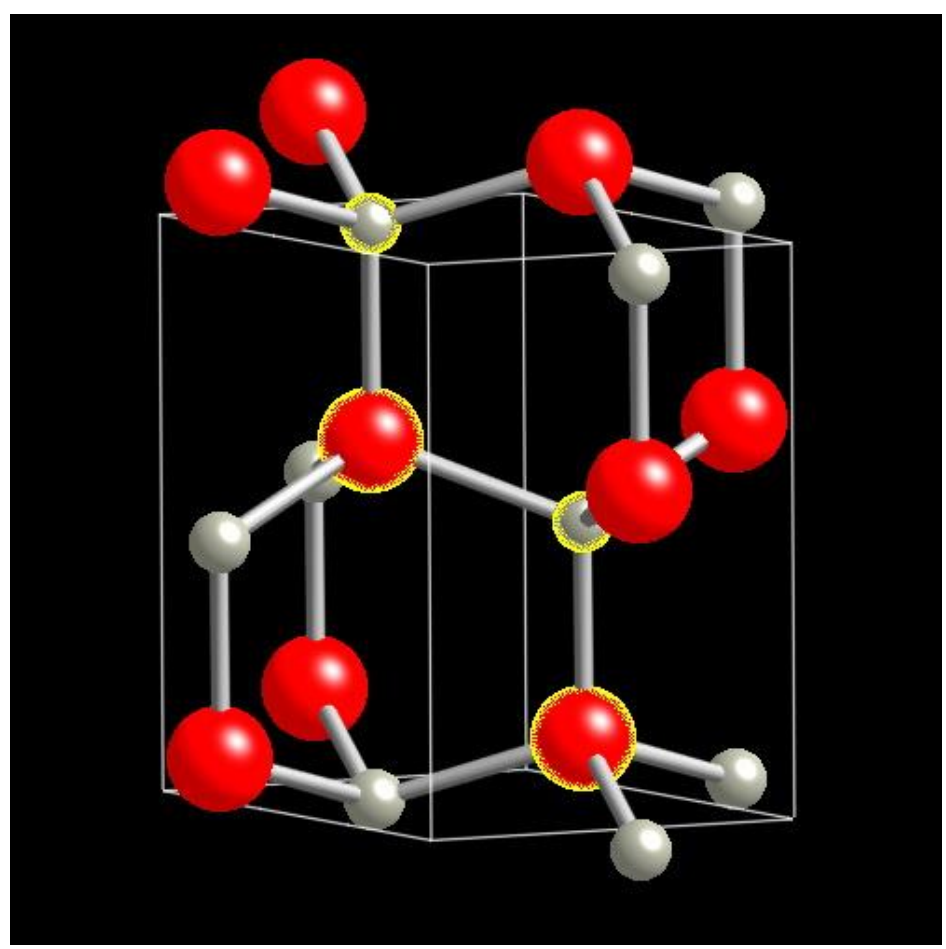

Figure 5.21 Crystal structure of wurtzite $\mathrm{ZnO}$. Grey color represents $\mathrm{Zn}$ atoms and red color represents $\mathrm{O}$ atoms. Highlighted atoms are inside the unit cell

$\mathrm{ZnO}$ which has the hexagonal wurtzite type lattice structure belongs to the $C_{6 v}^{4}$ symmetry group. ${ }^{27}$,

28 There are four atoms in the primitive unit cell and group theory predicted the following irreducible representation for the optical phonons at $\Gamma$ point of the Brillion zone. ${ }^{27-29}$

$$
\Gamma_{\mathrm{opt}}=A_{1}+E_{1}+2 E_{2}+2 B_{1}
$$


Both $A_{1}$ and $E_{1}$ modes are polar modes and exhibit the different frequencies for the transverseoptical (TO) and longitudinal-optical (LO) phonons. ${ }^{30}$ Also they are active in Raman and infrared spectroscopy. $B_{1}$ mode is a silent mode, it cannot be detected in either Raman or infrared spectroscopy. ${ }^{27,29}$ Since $E_{2}$ modes are non polar modes, they are not infrared active but they give two distinct frequencies due to the motion of $\mathrm{O}$ atoms and $\mathrm{Zn}$ sub lattice (Figure 5.22) in Raman spectra which are called as $E_{2}($ high $)$ and $E_{2}$ (low) modes respectively. ${ }^{27,29}$

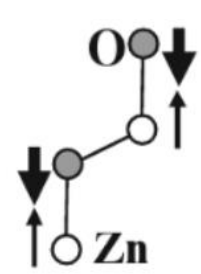

$\mathbf{A}_{1}$

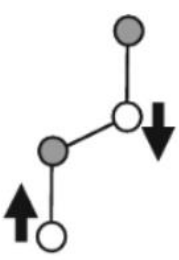

$\mathbf{B}_{1}{ }^{(1)}$

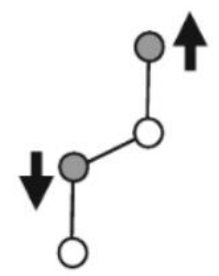

$B_{1}{ }^{(2)}$

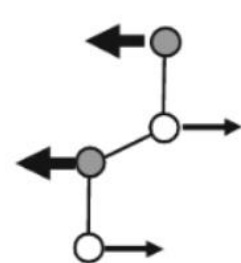

$\mathbf{E}_{1}$

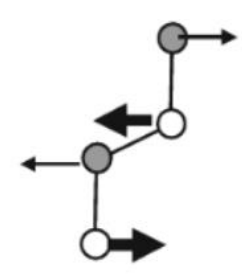

$E_{2}{ }^{(1)}$

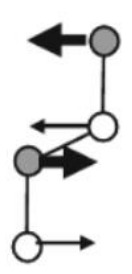

$E_{2}{ }^{(2)}$

Figure 5.22 Eigen vectors of the $\mathrm{ZnO}$ optical phonon modes. The bold arrows represent the dominating displacement vectors, while grey circles represent $\mathrm{Zn}$ atoms and white circles represent $\mathrm{O}$ atoms. Superscripts 1 and 2 showed the low and high frequencies.

The Raman active $E_{2}($ high $)$ mode of $\mathrm{ZnO}$ is temperature dependent and the temperature dependent linewidth broadening and frequency shifting for $E_{2}($ high $)$ are already reported. ${ }^{28,31-33}$ The linewidth broadening can be attributed to the temperature dependent phonon damping which is mainly due to the decay in to the lower energy phonons. ${ }^{32}$ In this work, linewidth broadening of $E_{2}($ high $)$ mode was used to estimate the local temperature of $\mathrm{ZnO}$.

Several phonon decay processes can be found in literature for $E_{2}(h i g h)$ mode. Samanta et $l^{32}$ reported a decay of $E_{2}(\mathrm{high})$ phonon mode $\left(441 \mathrm{~cm}^{-1}\right)$ in to three symmetric phonons (quadratic anharmonicity) with the frequency $\left(147 \mathrm{~cm}^{-1}\right)$. Cusco et $\mathrm{al}^{28}$ and Serrano et $a l^{34}$ used sum of two phonons $\left(250 \mathrm{~cm}^{-1}\right.$ and $\left.190 \mathrm{~cm}^{-1}\right)$ (cubic anharmonicity) and difference of two phonons (cubic 
anharmonicity) to explain the decay channel of $E_{2}(h i g h)$ phonon mode. Also Cusco et al ${ }^{28}$ reported that the rate of sum of two phonons decay is dominant over the difference of two phonons.

\subsubsection{Temperature dependent intensity dependent Raman spectra of $\mathrm{ZnO}$}

A Renishaw InVia Raman Microscope with $1.0 \mathrm{~cm}^{-1}$ resolution was used to collect the Raman spectra of $\mathrm{ZnO}$. The samples were excited with a $532 \mathrm{~nm}$ green laser (CL-532-100). A 5X magnification microscope objective was used in the experiments. Numerical aperture size was 0.12. The experimental laser spot size was measured using a calibration slide and was determined to be $\sim 13 \mu \mathrm{m}$. A Linkam high temperature stage (THMS 600) and temperature controller (TMS 94) were used to obtain temperature dependent Raman spectra of the samples in the temperature range of $25{ }^{\circ} \mathrm{C}$ to $450{ }^{\circ} \mathrm{C}$ with $25{ }^{\circ} \mathrm{C}$ steps under $2.6 * 10^{5} \mathrm{~W} / \mathrm{m}^{2}$ continuous wave (cw) $532 \mathrm{~nm}$ laser excitation (Figure 5.23). A series of Raman spectra were also collected for $\mathrm{ZnO}$ at different laser excitation intensities to measure the temperature change under laser illumination (Figure 5.24).

The $E_{2}($ high) mode of each Raman spectra was fitted in to Lorentzian line shape to determine the position and the full width half maximum (FWHM) or linewidth after the background subtraction. Both the linewidth and the position of the $E_{2}($ high) mode for temperature dependent Raman spectra were drastically changed with increasing temperature (Figure 5.25). The decreasing peak positon and increasing linewidth for $E_{2}$ (high) mode has been already reported. ${ }^{28,31}$ 


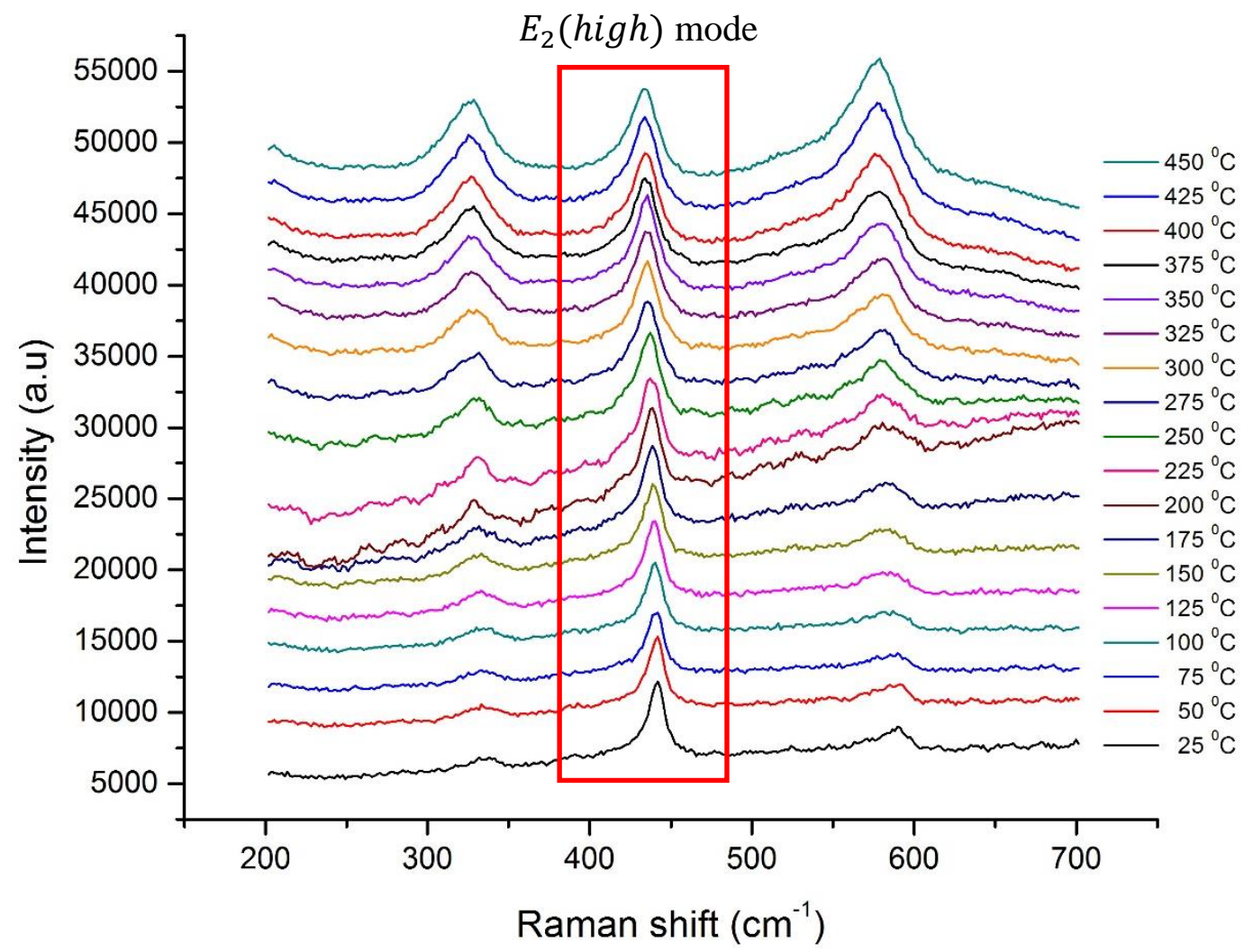

Figure 5.23 Raman spectra of $\mathrm{ZnO}$ at different temperatures

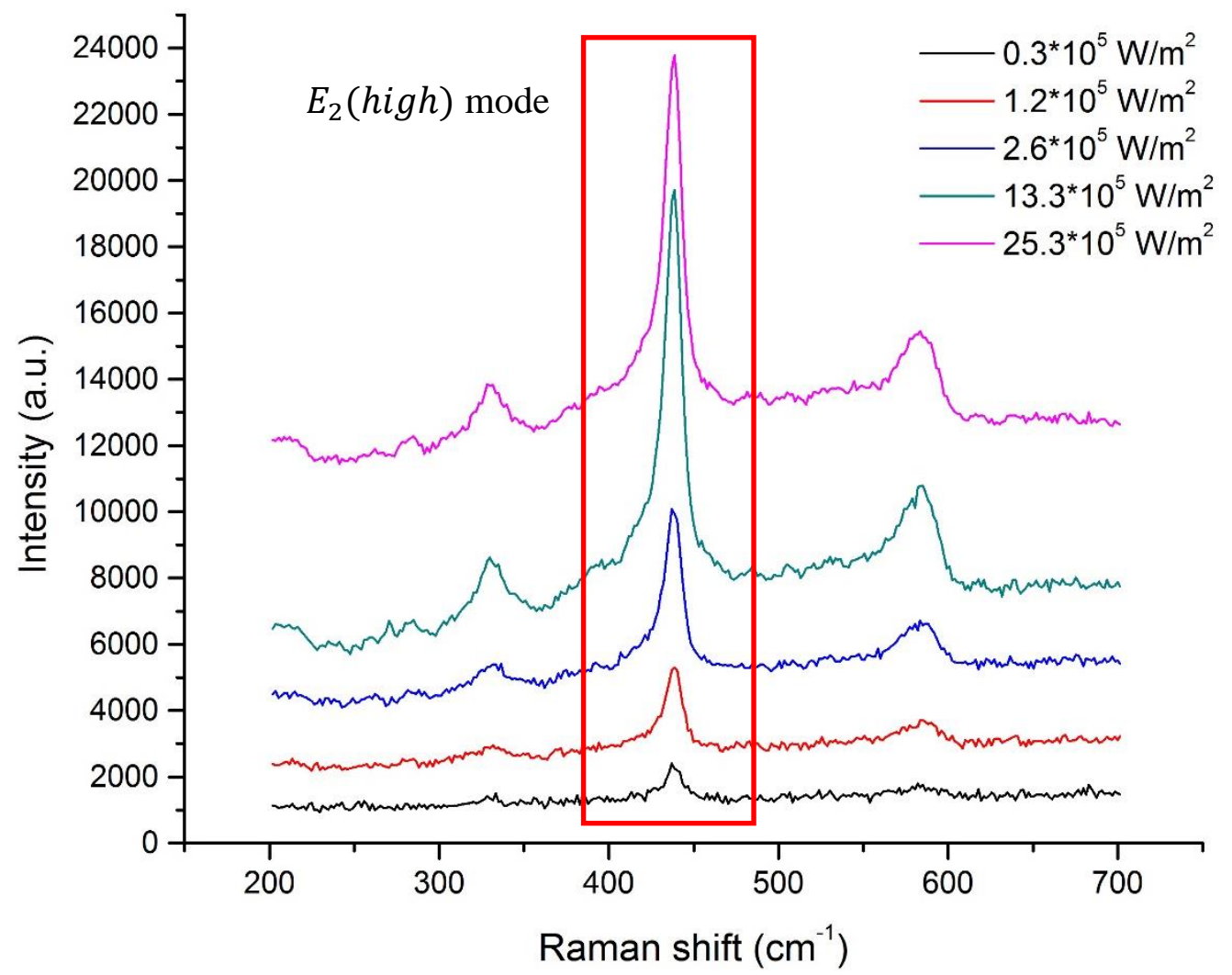

Figure 5.24 Raman spectra of $\mathrm{ZnO}$ at different laser intensities 
But for the laser intensity dependent Raman spectra at room temperature, both linewidth and position of the $E_{2}($ high) mode didn't show any considerable variation while increasing the laser intensity (Figure 5.26). Alim et $a l^{30}$ also previously reported the negligible laser heating effects for $E_{2}($ high $)$ mode of $\mathrm{ZnO}$ using the stokes and anti-stokes peak intensities. These results confirmed the negligible contribution of the laser intensity to the linewidth broadening of temperature dependent Raman spectra of $\mathrm{ZnO}$. In other words, there is no contribution of the laser intensity to the increasing temperature of $\mathrm{ZnO}$. The thermal expansion of the material affects the position of the Raman peaks but there is no effect on linewidth. ${ }^{31,32}$

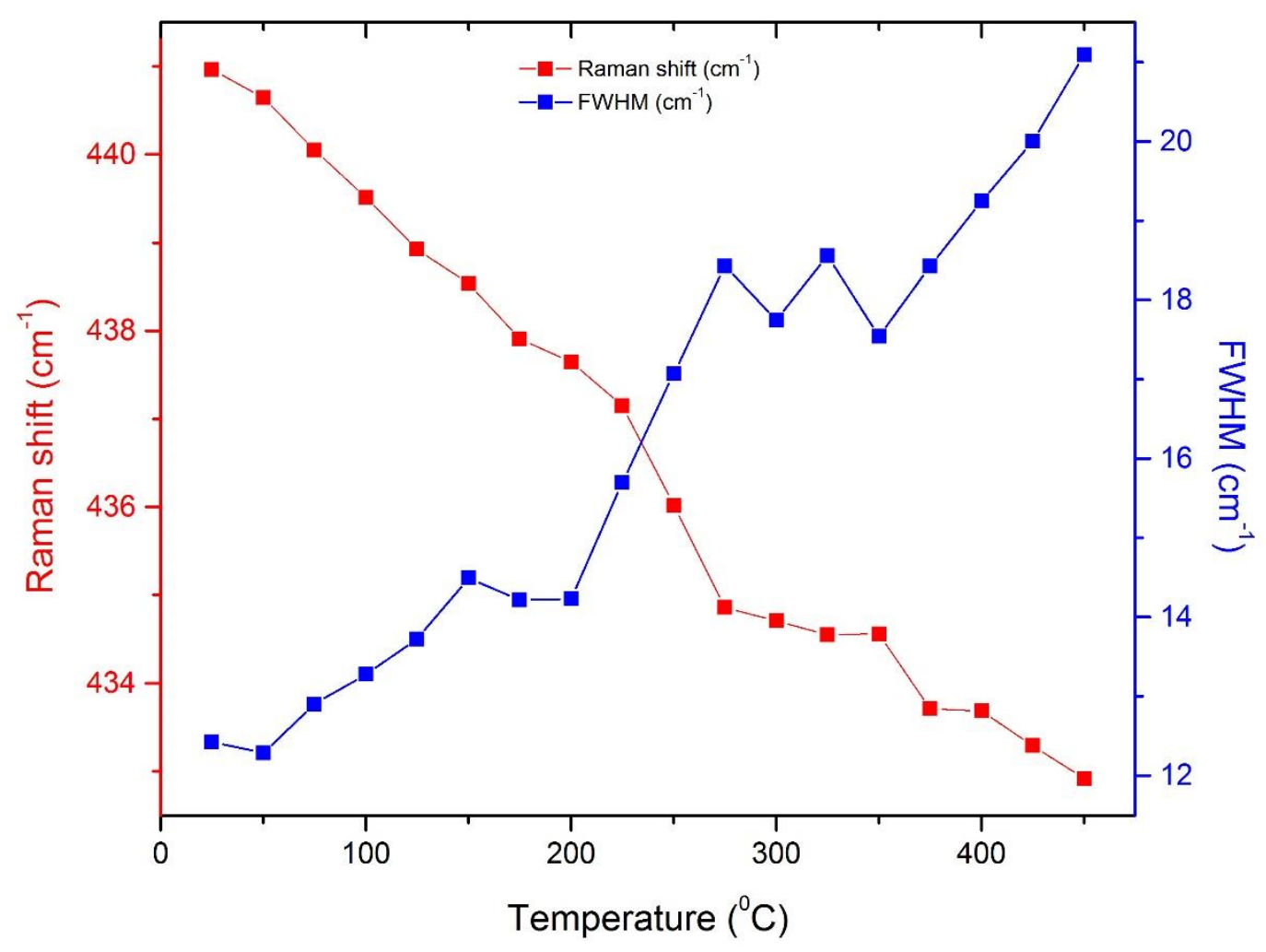

Figure 5.25 Raman shift and linewidth variation of $E_{2}($ high) mode of $\mathrm{ZnO}$ at different temperatures 


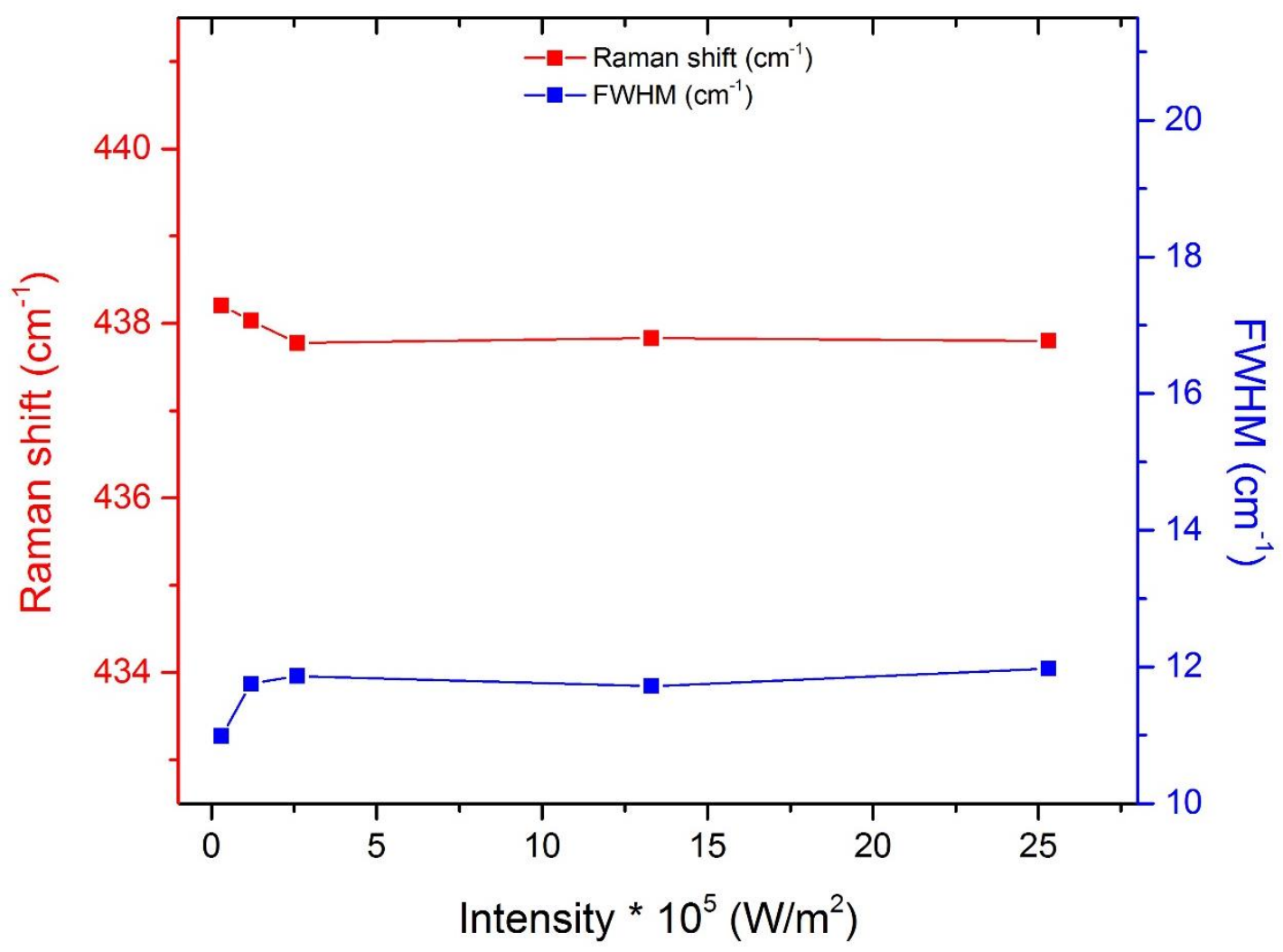

Figure 5.26 Raman shift and linewidth variation of $E_{2}(h i g h)$ mode of $\mathrm{ZnO}$ at laser intensities

\subsubsection{Theoretical model for the linewidth broadening of the $E_{2}($ high $)$ mode}

The temperature dependent linewidth of the optical phonons in $\mathrm{ZnO}$ is described in several previous reports ${ }^{27,} 28,31,32,34,35$ using the model derived by Menendez et al. ${ }^{36}$ The temperature dependence of linewidth of the $E_{2}($ high $)$ mode of $\mathrm{ZnO}$ can be explained using the following equation:

$T(T)=T_{0}+A\left(1+\sum_{j=1}^{2} n_{j}\left(\omega_{j}, T\right)\right)+B\left(1+\sum_{j=1}^{3} n_{j}\left(\omega_{j}, T\right)+n_{j}^{2}\left(\omega_{j}, T\right)\right)$

where $T(T)$ is the temperature dependent linewidth, $T_{0}$ is the background contribution to the linewidth due to impurity and/or defects scattering, $n_{j}\left(\omega_{j}, T\right)=\left[\exp \left(\frac{\hbar \omega}{k_{B} T}\right)\right]^{-1}$ is the Bose- 
Einestein distribution function and $A$ and $B$ are cubic and quadratic anharmonic coupling constants respectively. As mentioned in section 5.4.1, several proposed decay mechanisms for $E_{2}(h i g h)$ mode can be found in the literature. Yadav et $a l^{31}$ noted that cubic anharmonicity is dominating during the decay process but quadratic anharmonicity also is necessarily required. Therefore, in this work the both the sum of two phonons $\left(250 \mathrm{~cm}^{-1}\right.$ and $\left.190 \mathrm{~cm}^{-1}\right)$ (cubic anharmonicity) and sum of three symmetric phonons (quadratic anharmonicity) with the frequency $\left(147 \mathrm{~cm}^{-1}\right)$ were considered. The background contribution and anharmonic coupling constants were optimized using the experimental linewidth data and above theoretical equation. The optimized values for $T_{0}, A$ and $B$ found to be $2.56 \mathrm{~cm}^{-1}, 4.58$ and -0.08 respectively. This calibration curve (Figure 5.27) was used to estimate the local temperature of $\mathrm{Au}-\mathrm{ZnO}$ samples at different laser intensities.

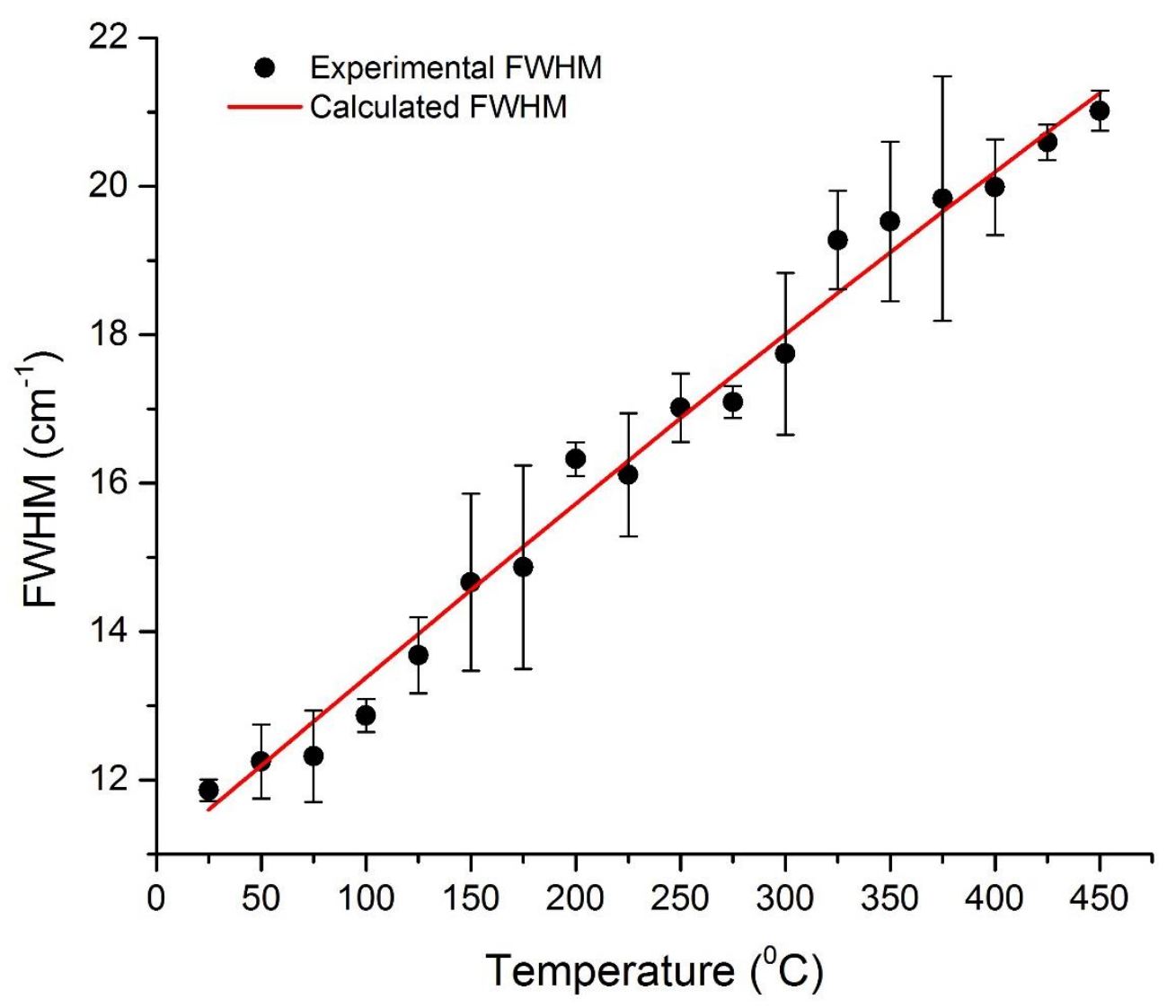

Figure 5.27 Linewidth of $\boldsymbol{E}_{\mathbf{2}}(\boldsymbol{h i g h})$ mode of $\mathrm{ZnO}$ as a function of temperature (black dots) under $2.6 * 10^{5} \mathrm{~W} / \mathrm{m}^{2} \mathrm{cw} 532 \mathrm{~nm}$ laser excitation. The red line is the fit based on the theoretical equation 


\subsubsection{Raman spectra of $\mathrm{ZnO}$ and $\mathrm{Au}-\mathrm{ZnO}$ at room temperature under different laser excitation intensities}

Raman spectra were collected for $\mathrm{Au}-\mathrm{ZnO}$ sample at room temperature under different laser intensities. The linewidth of the $E_{2}($ high $)$ mode was determined using the Lorentzian shape peak fitting. Neutral density filters were used to adjust the laser power beyond the limits of Raman spectrometer software. The linewidth of the $\mathrm{ZnO}$ shows a negligible variation under all the laser excitations (Figure 5.26). But the linewidth of the Au-ZnO showed a significant broadening when the laser intensity increased compared to the linewidth of the $\mathrm{ZnO}$ (Figure 5.28). Also, linewidth broadening is linearly dependent on the laser intensity. These results confirmed the heat generation (plasmonic heating) of $\mathrm{Au}-\mathrm{ZnO}$ sample under different laser intensities.

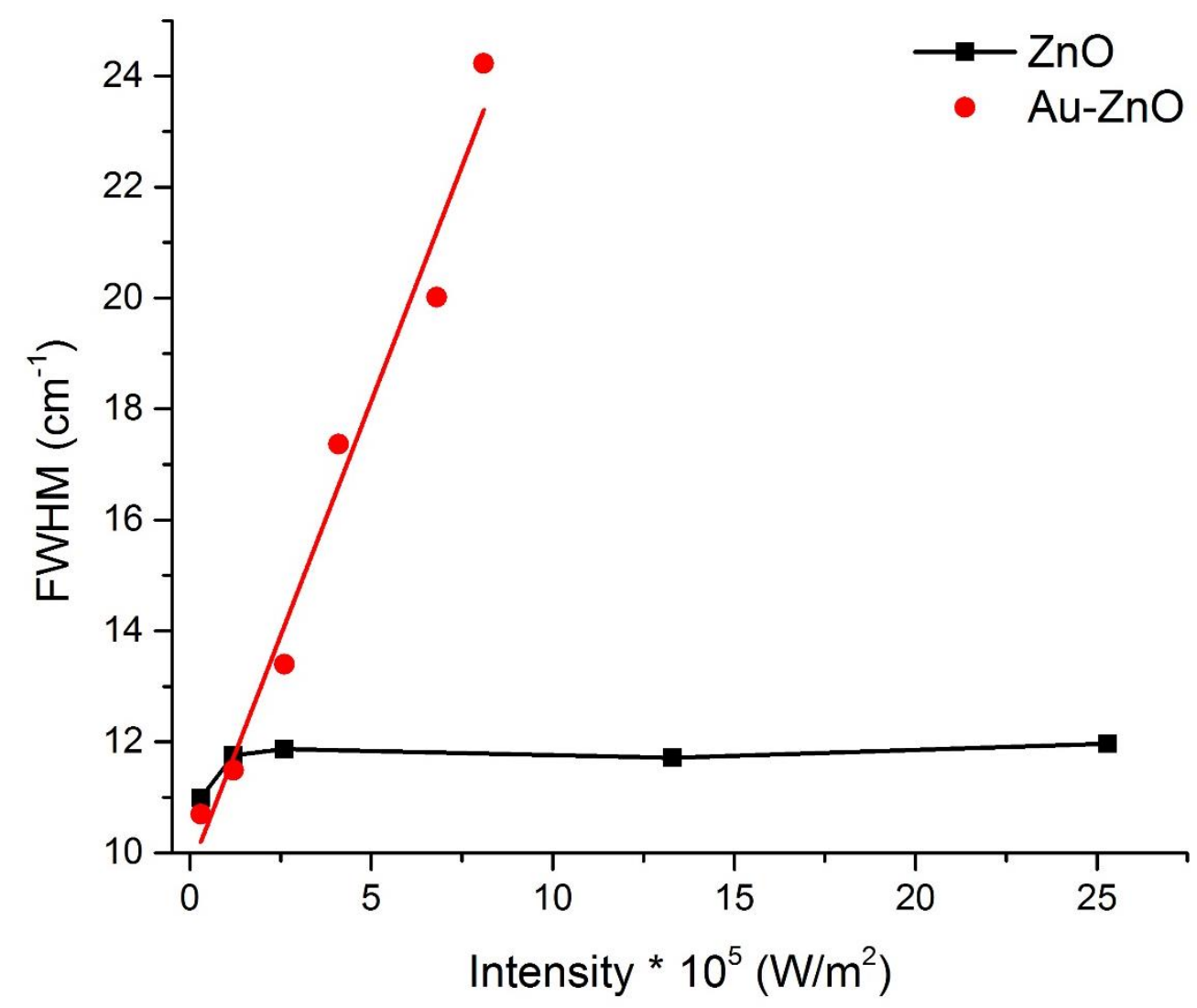

Figure 5.28 Linewidth of $E_{2}$ (high) mode of $\mathrm{ZnO}$ (black squares) and $\mathrm{Au}-\mathrm{ZnO}$ (red dots) as a function of different intensities of $\mathrm{cw} 532 \mathrm{~nm}$ laser excitation. The red line is a linear fit of the linewidth variation of the $\mathrm{Au}-\mathrm{ZnO}$ 
The steady state local temperature of the $\mathrm{Au}-\mathrm{ZnO}$ at given laser intensity was determined using the calibration curve (Figure 5.27). The calibration curve was slightly extrapolated to estimate the local temperature at laser intensity $8.0 * 10^{5} \mathrm{~W} / \mathrm{m}^{2}$. Table 5.3 and Figure 5.29 show the estimated local temperature of $\mathrm{Au}-\mathrm{ZnO}$ at different laser intensities. The local temperature linearly increases as laser intensity increases which is well agreed with the Equation 5.7. But due to the clustering of $\mathrm{Au}$ nanoparticles this estimated local temperature can't be compared with the calculated temperature increases in Figures 5.4 and 5.5. Since plasmonic heating is highly localized, this estimated local temperature reflects an average steady state temperature of the $\mathrm{ZnO}$ surface. The local temperature in close proximity to the $\mathrm{Au}-\mathrm{ZnO}$ interface could be significantly higher than the estimated local temperature.

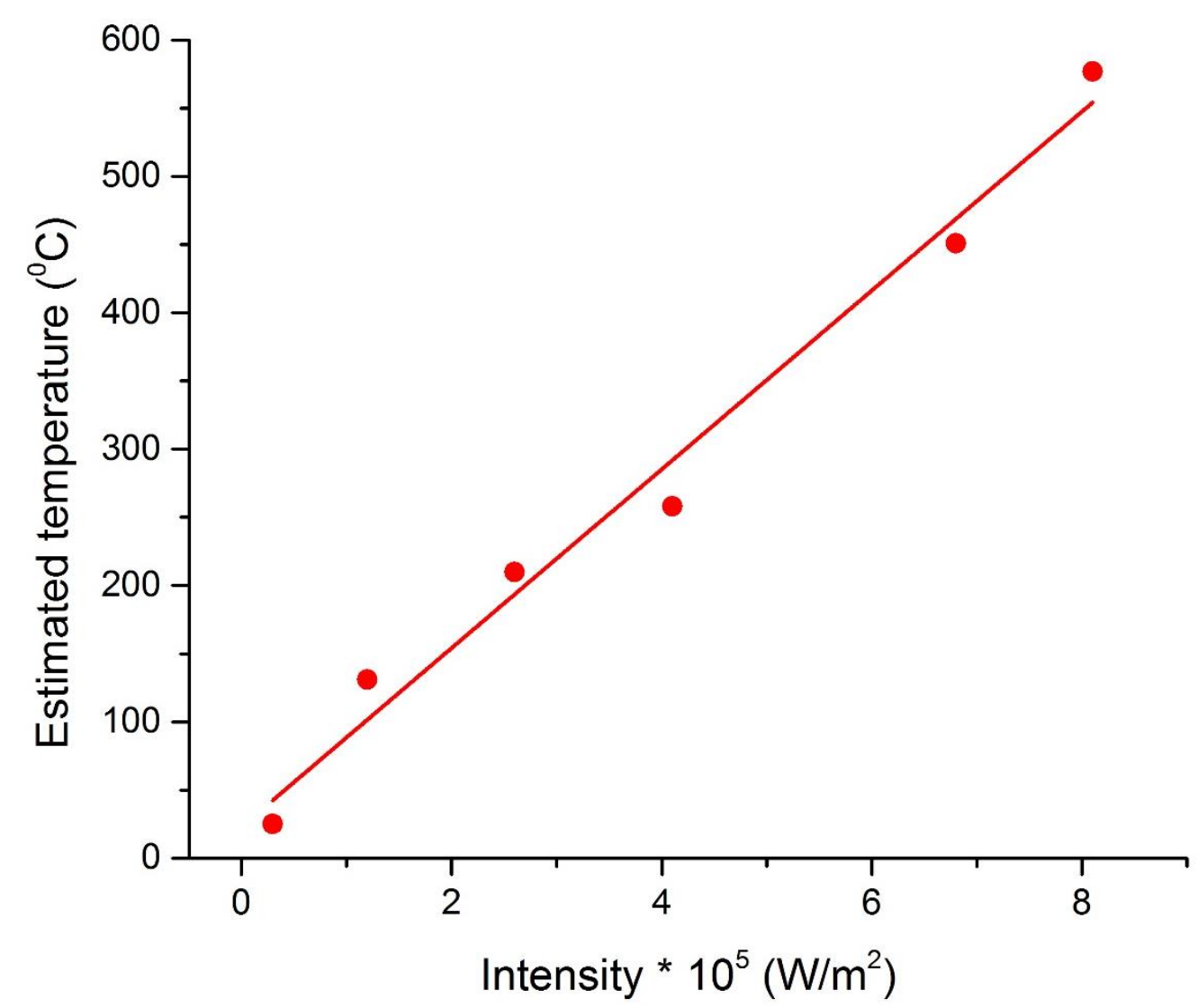

Figure 5.29 Estimated local temperature of $\mathrm{Au}-\mathrm{ZnO}$ under different laser intensities 


\section{$5.6 \mathrm{CO}_{2}$ reduction reactions}

A gas-tight photocatalysis cell was constructed using a stainless steel spacer (MDC Vacuum Products) with conflat flange components and fitted with two inlet/outlet valves, one gas chromatography (GC) sampling port (HP 5890) connected via Swagelok fittings, and two UV quartz viewports. The two UV quartz viewports along the laser irradiation direction ensured that no metal part of the photocatalysis cell would be illuminated by the $532 \mathrm{~nm}$ laser. The volume of the photocatalysis cell was $40 \mathrm{~mL}$. A watch glass was placed at the bottom of the UV quartz viewport of the photocatalysis cell, on which 10 to $15 \mathrm{mg}$ of the catalyst samples were loaded for photocatalysis reactions. After a new septum was placed in the sampling port and a catalyst sample was loaded, the photocatalysis cell was purged with $25 \% \mathrm{CO}_{2}+75 \% \mathrm{H}_{2}$ of gas mixture for 20 minutes. The photocatalysis cell was subsequently irradiated through the top quartz viewport using a $c w 532 \mathrm{~nm}$ laser laser (Figure 5.30). The laser spot size was $\sim 150 \mu \mathrm{m}$ and illumination time was $5 \mathrm{hr}$. Gas chromatography (GC) analysis of photoreduction products was performed using a Perkin Elmer Clarus 600 Gas Chromatograph with a heated sample inlet. Sample detection was achieved using both a thermal conductivity detector and a flame ionization detector with methanizer, joined in sequence. Gas samples were injected manually using a $5 \mathrm{~mL}$ gas-tight syringe. 

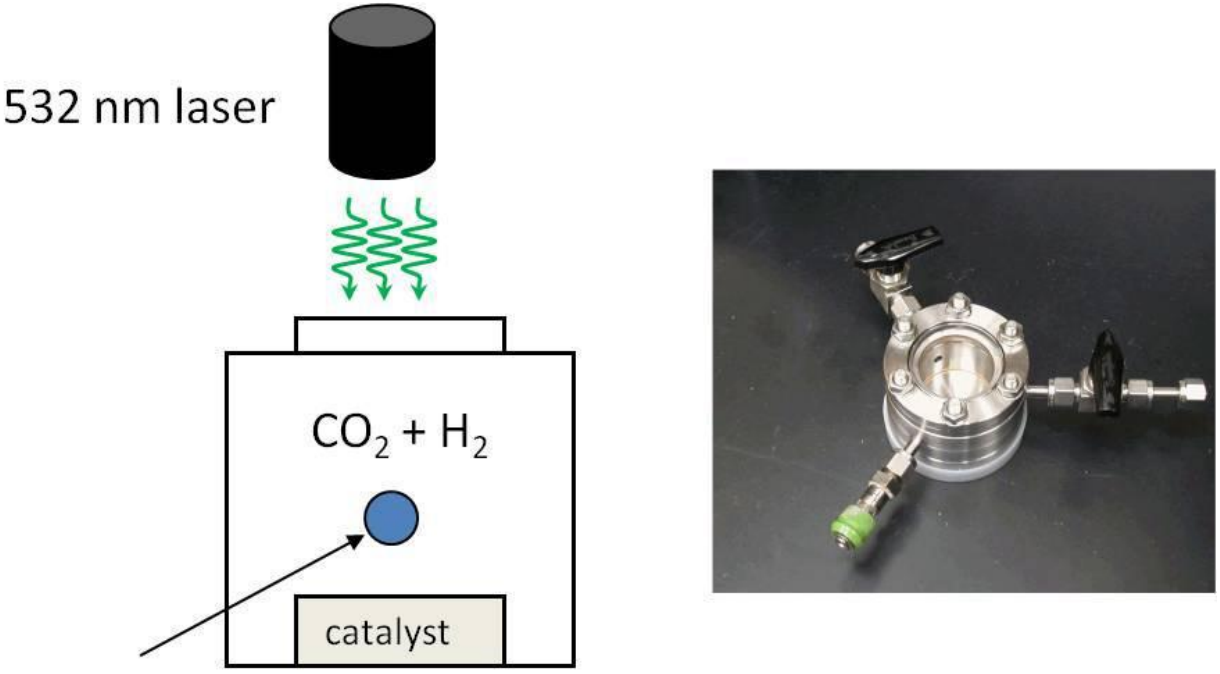

\section{Gas sampling port for}

\section{GC analysis}

Figure 5.30 The schematic diagram of laser illumination and homemade photocatalysis cell

The $532 \mathrm{~nm} c w$ laser was used for plasmonic heating assisted $\mathrm{CO}_{2}$ reduction which allowed to directly match the intensities used in Raman experiments to estimate the local temperatures of Au$\mathrm{ZnO}$. After the laser illumination, two reaction products $\left(\mathrm{CH}_{4}\right.$ and $\left.\mathrm{CO}\right)$ were detected using the GC. Then $\mathrm{CO}_{2}$ conversion rate (Figure 5.31) and product generation rates (Figures 5.32 and 5.33) were calculated based on moles of products, amount of catalyst and illumination time. The reaction rate $4.22 \mu \mathrm{mol} / \mathrm{g} / \mathrm{h}$ of the $\mathrm{Au}-\mathrm{ZnO}$ catalyst under $\sim 8 * 10^{5} \mathrm{~W} / \mathrm{m}^{2} c w 532 \mathrm{~nm}$ laser illumination is nearly 4 times higher than the previously observed using $\mathrm{PbS} / \mathrm{Cu} / \mathrm{TiO}_{2}$ catalysts under visible light excitation in a similar reaction cell using the same gas product detection method ${ }^{37}$ The control experiments with $\mathrm{ZnO}$ and $\mathrm{SiO}_{2}$ didn't show any considerable activity even at the highest laser intensity (Figure 5.31). 


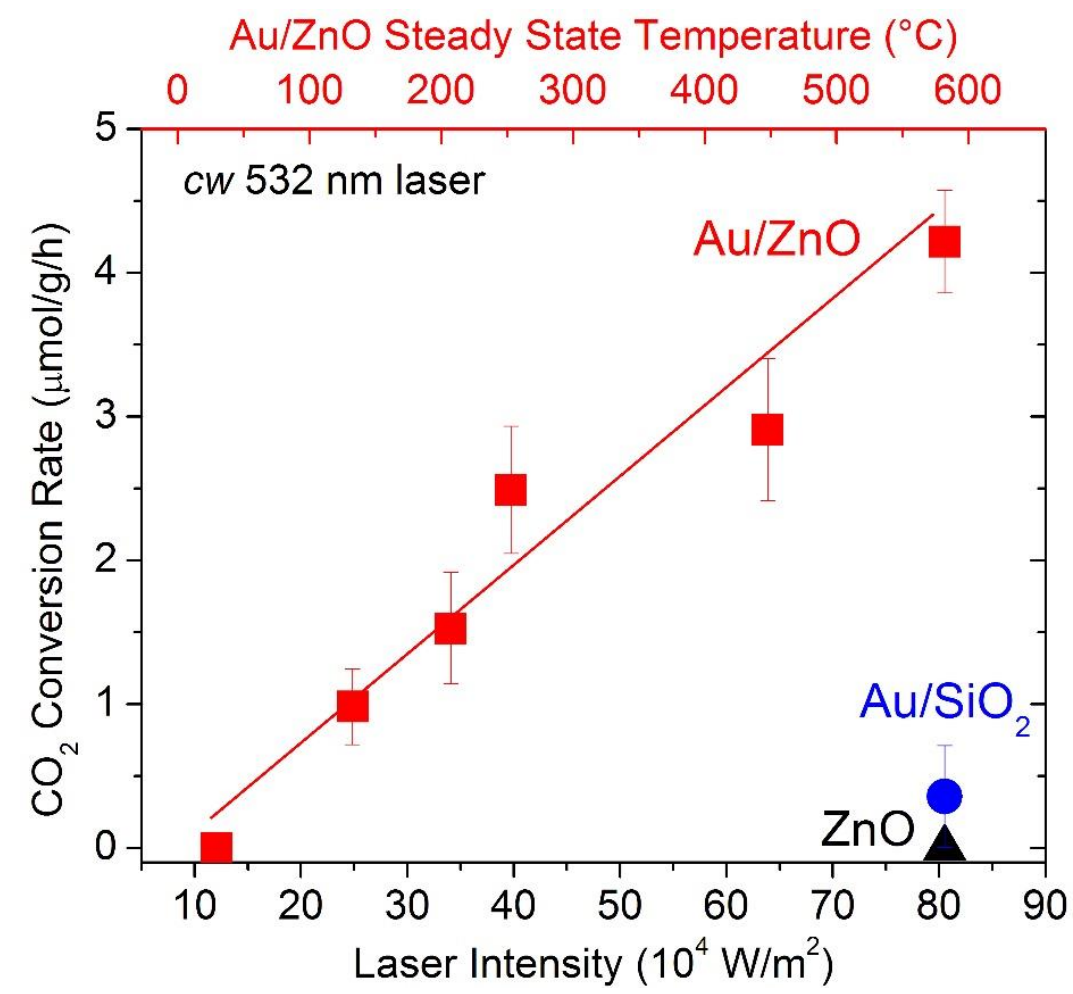

Figure 5.31 $\mathrm{CO}_{2}$ conversion rate at different laser intensities with $\mathrm{Au}-\mathrm{ZnO}$, with the corresponding local temperature due to the plasmonic heating as the top $x$-axis

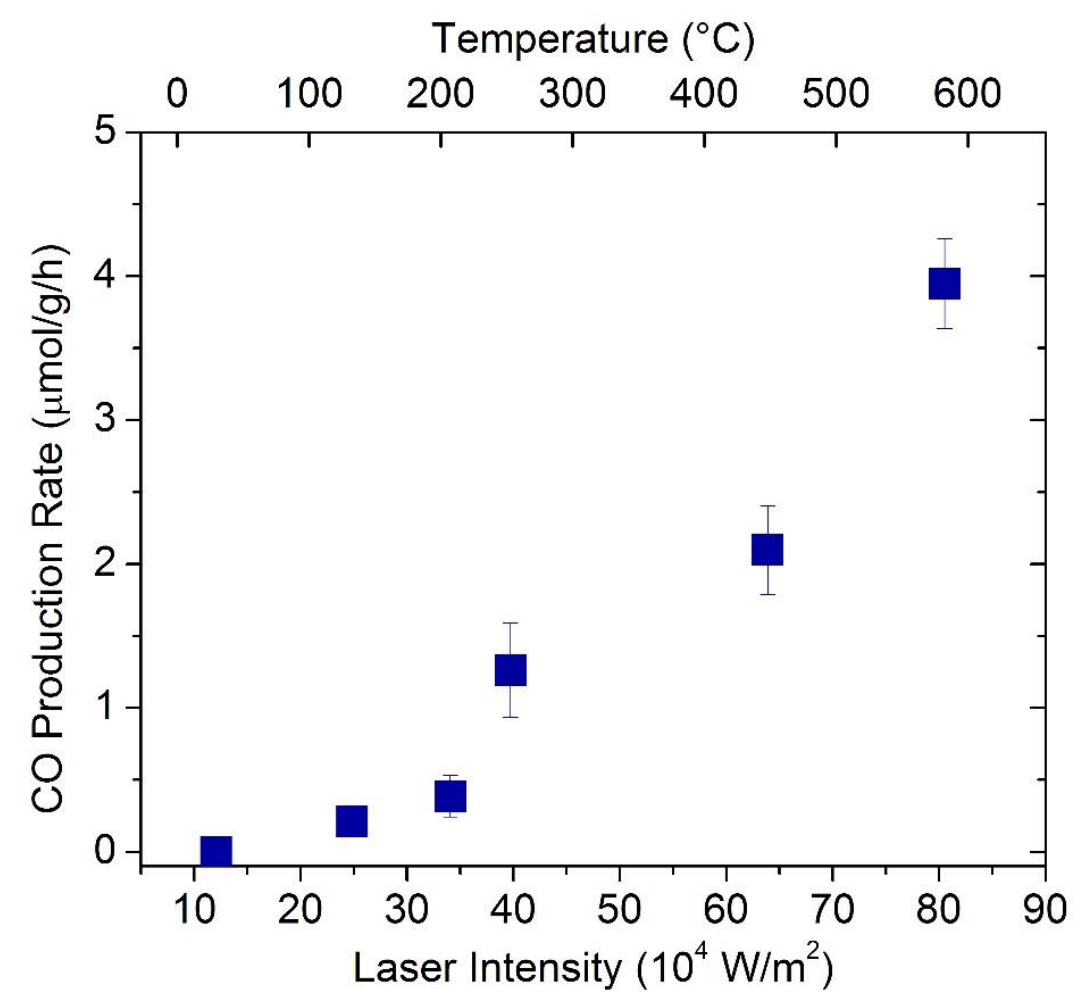

Figure 5.32 $\mathrm{CO}$ production rate at different laser intensities with $\mathrm{Au}-\mathrm{ZnO}$, with the corresponding local temperature due to the plasmonic heating as the top $x$-axis 


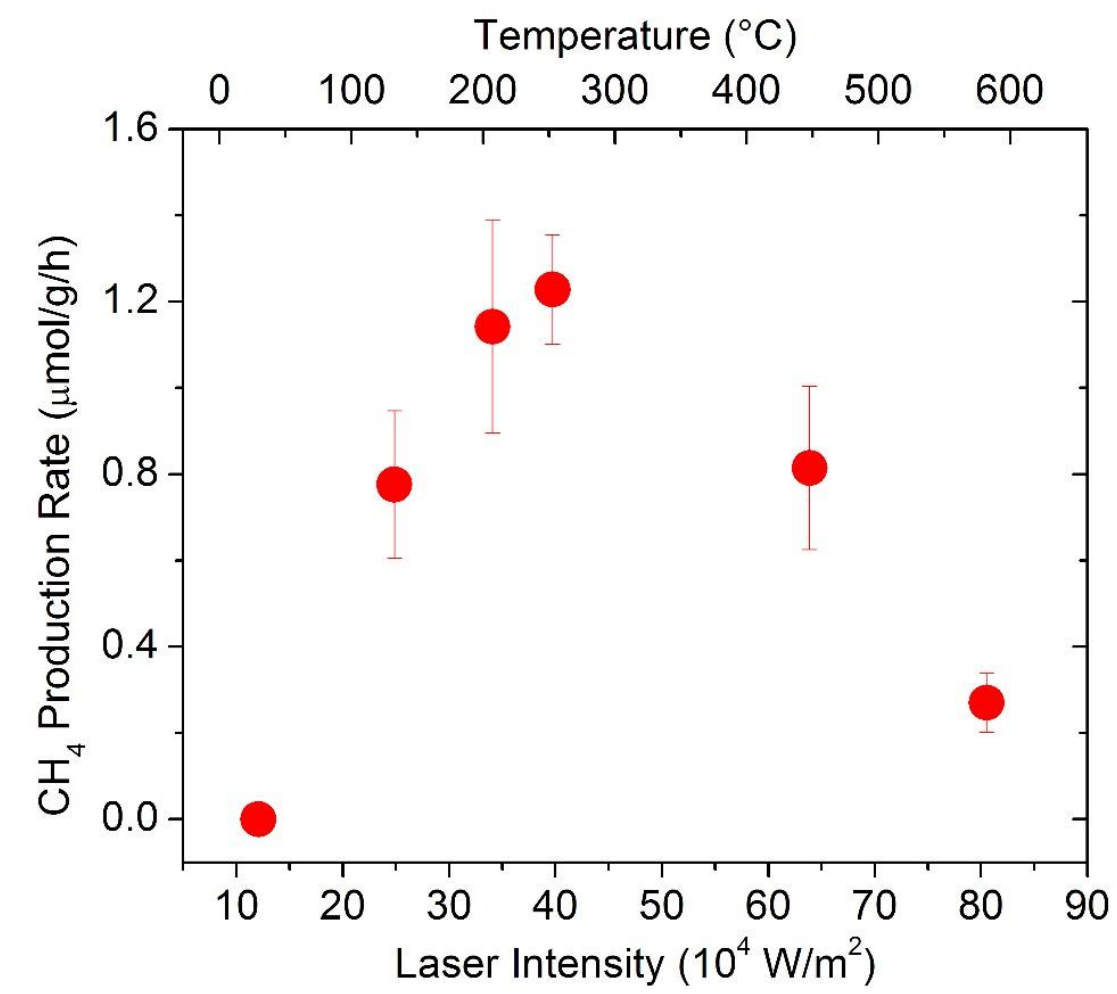

Figure 5.33 $\mathrm{CH}_{4}$ production rate at different laser intensities with $\mathrm{Au}-\mathrm{ZnO}$, with the corresponding local temperature due to the plasmonic heating as the top $x$-axis

Wang et $a l^{38}$ suggested following four possible reaction schemes for $\mathrm{CO}_{2}$ conversion reactions using the thermodynamic calculations.

Reaction scheme 1: $\mathrm{CO}_{2}+\mathrm{H}_{2} \leftrightarrows \mathrm{CO}+\mathrm{H}_{2} \mathrm{O}$ (Reversed Water Gas Shift (RWGS))

Reaction scheme 2: $\mathrm{CO}_{2}+\mathrm{H}_{2} \leftrightarrows \mathrm{CO}+\mathrm{H}_{2} \mathrm{O}$ (RWGS); $\mathrm{CO}_{2}+3 \mathrm{H}_{2} \leftrightarrows \mathrm{CH}_{3} \mathrm{OH}+\mathrm{H}_{2} \mathrm{O}$ (Methanol synthesis)

\section{Reaction scheme 3: $\mathrm{CO}_{2}+\mathrm{H}_{2} \leftrightarrows \mathrm{CO}+\mathrm{H}_{2} \mathrm{O}$ (RWGS); $\mathrm{CO}+3 \mathrm{H}_{2} \leftrightarrows \mathrm{CH}_{4}+\mathrm{H}_{2} \mathrm{O}$ (CO- methanation)}

Reaction scheme 4: $\mathrm{CO}_{2}+\mathrm{H}_{2} \leftrightarrows \mathrm{CO}+\mathrm{H}_{2} \mathrm{O}$ (RWGS); $\mathrm{CO}_{2}+3 \mathrm{H}_{2} \leftrightarrows \mathrm{CH}_{3} \mathrm{OH}+\mathrm{H}_{2} \mathrm{O}$ (Methanol synthesis); $\mathrm{CO}+3 \mathrm{H}_{2} \leftrightarrows \mathrm{CH}_{4}+\mathrm{H}_{2} \mathrm{O}$ (CO-methanation) 
The $3^{\text {rd }}$ reaction scheme is consistent with the detected products for $\mathrm{CO}_{2}$ reductions reactions using the $\mathrm{Au}-\mathrm{ZnO}$ in this work. First $\mathrm{CO}_{2}$ react with $\mathrm{H}_{2}$ in the presence of plasmonically heated $\mathrm{ZnO}$ (photocatalyst) and generate $\mathrm{CO}$ and $\mathrm{H}_{2} \mathrm{O}$ (Reversed water gas shift). Then $\mathrm{CO}$ further react with $\mathrm{H}_{2}$ to generate the $\mathrm{CH}_{4}$ ( $\mathrm{CO}$ methanation). Both reactions (Reversed water gas shift and $\mathrm{CO}$ methanation) are thermally driven and production rates depend on the reaction temperature (Figures 5.32 and 5.33). The temperature programmed reaction studies were performed in order to further confirm the thermal driven catalytic activity of $\mathrm{ZnO}$.

\subsubsection{Temperature programmed reactions}

Temperature programmed reaction was performed using a Micromeritics Autochem 2950 HP equipped with a Thermostar mass spectrometer (MS) to verify that $\mathrm{CO}$ and $\mathrm{CH}_{4}$ were thermochemically produced using the $\mathrm{ZnO}$ and $\mathrm{Au}-\mathrm{ZnO}$ catalysts. $\mathrm{ZnO}$ was dispersed in a $\mathrm{pH} \sim 2$ $\mathrm{HCl}$ solution, dried, and calcined at $300{ }^{\circ} \mathrm{C}$ in air for $1 \mathrm{~h}$ before cooling to room temperature, to follow the similar steps which were used for synthesis of $\mathrm{Au}-\mathrm{ZnO}$ heterostructures. $\mathrm{Au}-\mathrm{SiO}_{2}$ and $\mathrm{SiO}_{2}$ were also studied as control experiments. The $25 \% \mathrm{CO}_{2}+75 \% \mathrm{H}_{2}$ of gas mixture $\left(100 \mathrm{~cm}^{3}\right.$ $\mathrm{min}^{-1}$ ) was introduced to $300 \mathrm{mg}$ of the samples while the reactor pressure was kept at atmospheric pressure. The catalyst temperature was subsequently raised linearly at the rate of $10{ }^{0} \mathrm{C} \mathrm{min}^{-1}$ from

room temperature to $650{ }^{\circ} \mathrm{C}$. Fragments of the gas components in the outlet stream of the reactor $\left(\mathrm{H}_{2}, \mathrm{CH}_{4}, \mathrm{H}_{2} \mathrm{O}, \mathrm{CO}, \mathrm{CH}_{3} \mathrm{OH}\right.$ and $\left.\mathrm{CO}_{2}\right)$ were monitored using a ThermoStar mass spectrometer.

These control experiments were done in the dark and both $\mathrm{ZnO}$ and $\mathrm{Au}-\mathrm{ZnO}$ show similar catalytic activity as laser illumination $\mathrm{CO}_{2}$ reduction experiments by producing the $\mathrm{CO}$ and $\mathrm{CH}_{4}$ from $\mathrm{CO}_{2}$ reduction. The production rates for both $\mathrm{CO}$ and $\mathrm{CH}_{4}$ are qualitatively identical to the laser 
illumination $\mathrm{CO}_{2}$ reduction experiments (Figures 5.34 and 5.35). The generation of $\mathrm{CO}$ starts just below $150{ }^{\circ} \mathrm{C}$ in laser illumination experiments while generation of $\mathrm{CO}$ in temperature programmed dark experiments start around $425{ }^{\circ} \mathrm{C}$. Also $\mathrm{CH}_{4}$ production starts below $100{ }^{\circ} \mathrm{C}$ for laser illumination experiments while $\mathrm{CH}_{4}$ production in temperature programmed dark experiments start around $275{ }^{0} \mathrm{C}$. The increasing $\mathrm{CO}$ production rate with the increasing temperature also well agrees with laser illumination experiments. The increasing and decreasing behavior of the $\mathrm{CH}_{4}$ production rate is also observed in both laser illumination experiments and temperature programmed dark experiments. The maximum $\mathrm{CH}_{4}$ production rate was achieved around $250{ }^{\circ} \mathrm{C}$ and $425{ }^{\circ} \mathrm{C}$ for laser illumination experiments and temperature programmed dark experiments respectively. Also $\mathrm{Au}-\mathrm{SiO}_{2}$ didn't show considerable activity compared to the $\mathrm{ZnO}$ and $\mathrm{Au}-\mathrm{ZnO}$ for temperature programmed dark experiments respectively (Figures 5.34 and 5.35). These results can be identified as a strong evidence to confirm the thermally driven catalytic activity of $\mathrm{ZnO}$ for $\mathrm{CO}_{2}$ reduction which follows the $3^{\text {rd }}$ reaction scheme. Also, this indicates that plasmonic heating is occurring and it can drive purely thermal catalytic reactions. By comparing the results of the laser illumination experiments and temperature programmed dark experiments; one can point out that only difference is the shift in the temperature axis. The Raman spectra based local temperature estimation most probably underestimates the local temperature. 


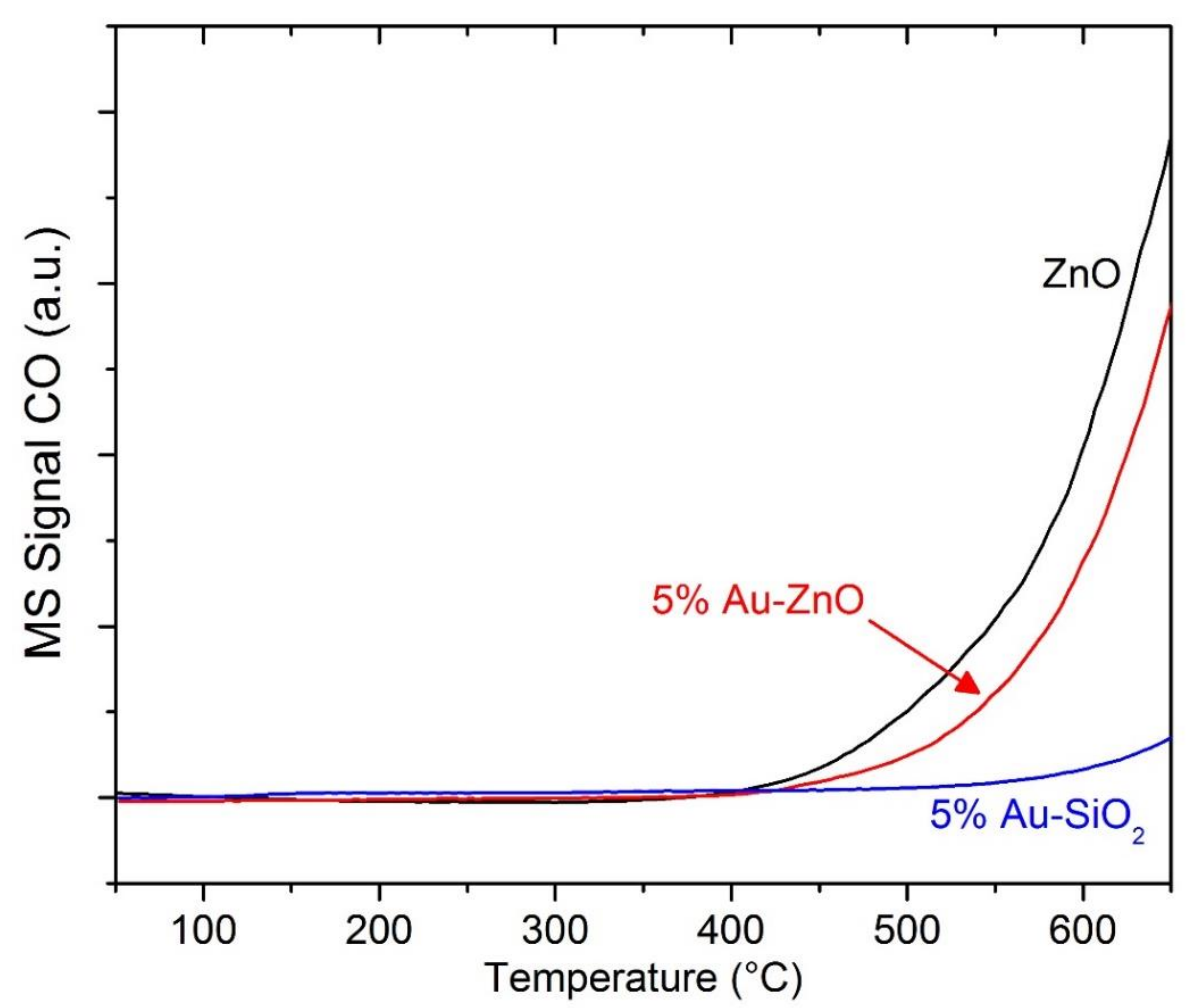

Figure 5.34 $\mathrm{CO}$ production detected by a mass spectrometer as function of temperature for $\mathrm{ZnO}, \mathrm{Au}-\mathrm{ZnO}$ and $\mathrm{Au}-\mathrm{SiO}_{2}$

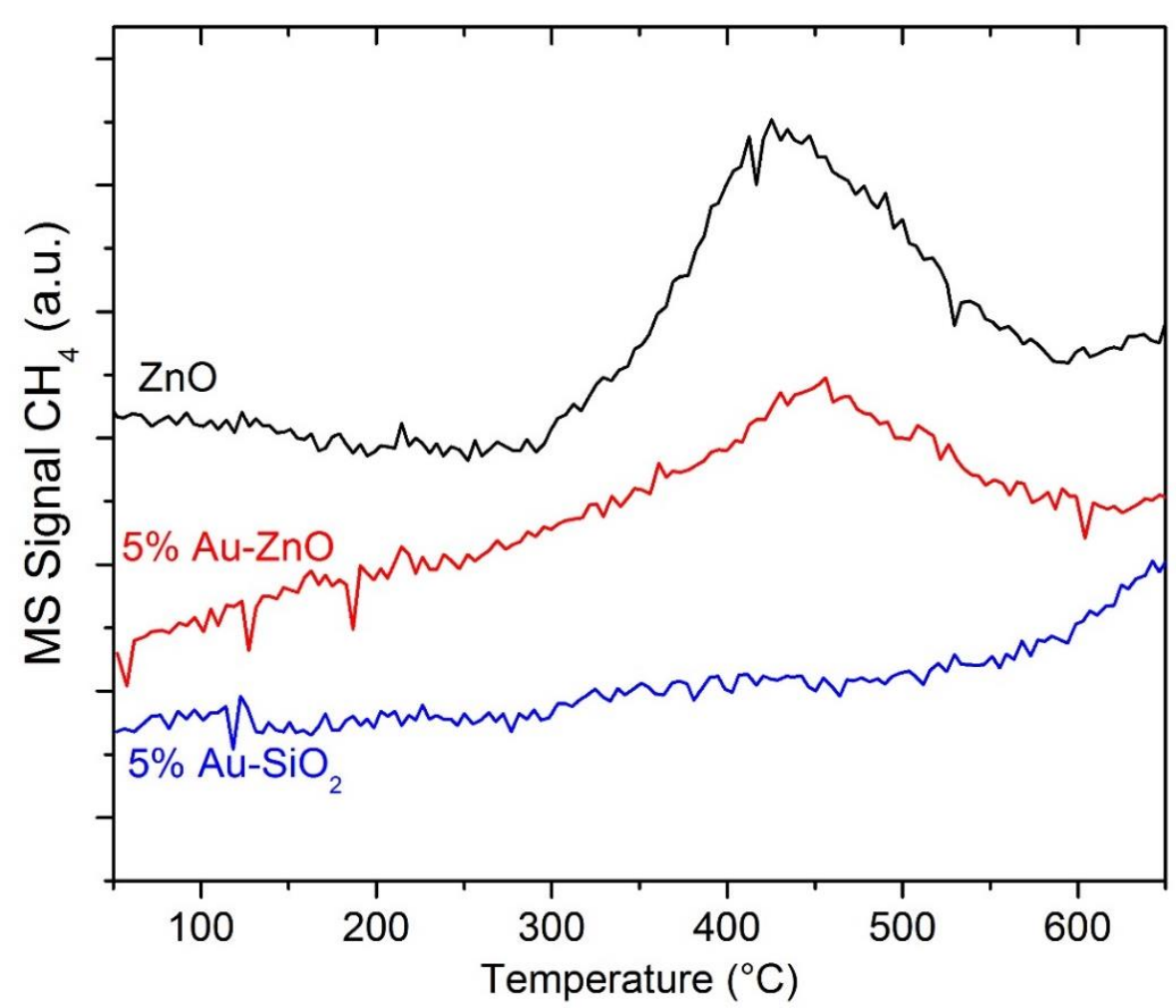

Figure 5.35 $\mathrm{CH}_{4}$ production detected by a mass spectrometer as function of temperature for $\mathrm{ZnO}, \mathrm{Au}-\mathrm{ZnO}$ and $\mathrm{Au}-\mathrm{SiO}_{2}$ 


\subsubsection{Plasmonic heating assisted catalytic activity of Au-ZnO}

Based on Raman spectral analysis, laser illumination experiments and temperature programmed dark experiments confirm the plasmonic heating assisted purely thermal driven $\mathrm{CO}_{2}$ reduction. The nanoparticle size distribution, nanoparticle densities, available reaction channels, illumination light intensity and its resonance with surface plasmons can be identified as the main parameters which directly affect the production efficiency. Also, it is very important to have a strong resonance of surface plasmon with illumination light to generate the maximum heat. The surface plasmon resonance of Au nanoparticles in this work $(\sim 538 \mathrm{~nm})$ were well resonated with $532 \mathrm{~nm}$ laser illumination and it could be one reason to have a higher $\mathrm{CO}_{2}$ conversion rate. Since illumination light intensity is directly proportional to the local temperature increase (Equation 5.7, Figures 5.4 and 5.29) the local temperature of the catalyst can be easily changed by just changing the illumination light intensity. The temperature dependent product selectivity was confirmed by both laser illumination experiments and temperature programmed dark experiments (Figures 5.32, $5.33,5.34,5.35)$. This is very important for large scale industrial applications and it allows controlling the amount of products as it needed. For an example; at the lower intensities it can generate more $\mathrm{CH}_{4}$ than the $\mathrm{CO}$ but at higher intensities it can generate more $\mathrm{CO}$ than the $\mathrm{CH}_{4}$. Also it is possible to get 1:1 ratio of $\mathrm{CO}$ and $\mathrm{CH}_{4}$ at middle intensities (Figure 5.36). 


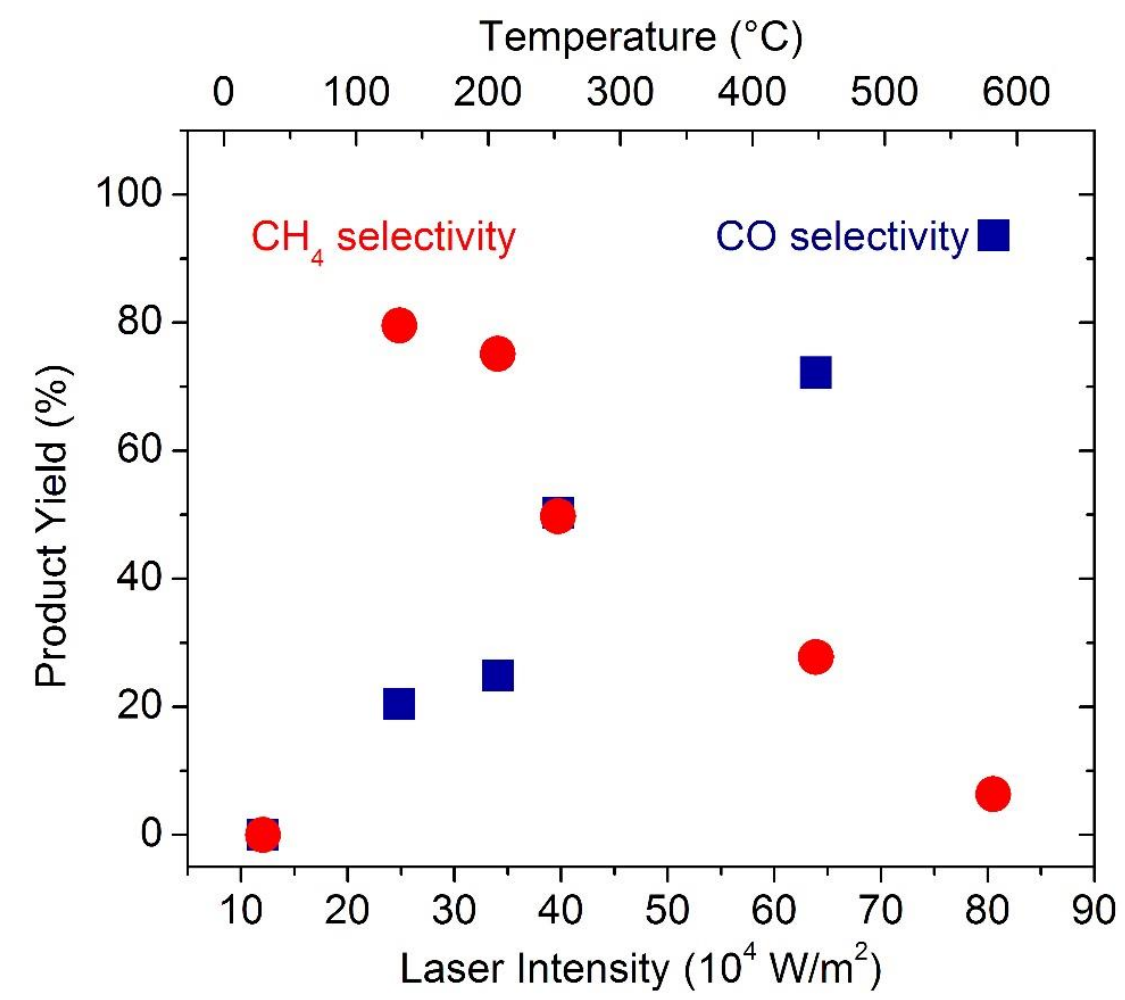

Figure 5.36 The experimental CO (blue squares) and CH4 (red circles) selectivity as a function of laser intensity and corresponding catalyst temperature in top $x$-axis

Since long term catalytic stability is an important factor for large scale, this $\mathrm{Au}-\mathrm{ZnO}$ catalyst was used to run five consecutive $\mathrm{CO}_{2}$ reduction cycles. The catalyst was illuminated for 5 hours using a $532 \mathrm{~nm} \mathrm{cw}$ laser with the intensity of $8.0 * 10^{5} \mathrm{~W} / \mathrm{m}^{2}$ (maximum laser intensity used for $\mathrm{CO}_{2}$ reduction reactions). At the end of each illumination cycle photocatalysis cell was purged with new $25 \% \mathrm{CO}_{2}+75 \% \mathrm{H}_{2}$ gas mixture for next illumination. The variation of $\mathrm{CO}_{2}$ conversion rate was $<15 \%$ in non-systematic fashion (Figure 5.37). This high stability of the $\mathrm{Au}-\mathrm{ZnO}$ can be attributed to the thermally driven $\mathrm{CO}_{2}$ conversion. In most photo-redox systems, oxidative corrosion of the catalyst and depletion of hole scavengers directly impact the long-term stability of the catalyst. Also, this confirms that the laser induced damage does not occur under the $532 \mathrm{~nm}$ $c w$ laser illumination. 


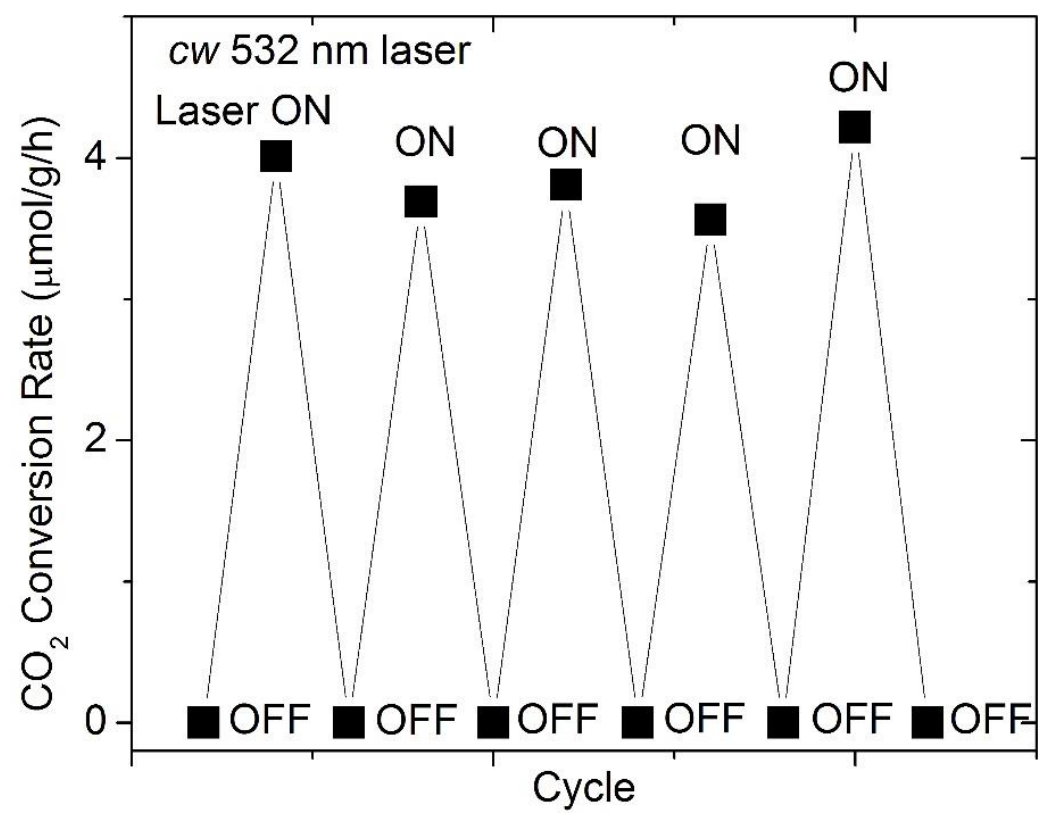

Figure 5.37 The $\mathrm{CO}_{2}$ conversion rate for $\mathrm{Au}-\mathrm{ZnO}$ catalyst after 5 hour laser illumination cycle

\subsection{Conclusion}

In this work, plasmonic heating assisted efficient $\mathrm{CO}_{2}$ conversion was proved and this method has a higher potential to develop in large scale applications. In addition to that this plasmonic heating effect of $\mathrm{Au}-\mathrm{ZnO}$ can be used for several other applications such as steam generation and drug delivery. The Au-ZnO sample reached close to $\sim 600{ }^{\circ} \mathrm{C}$ under $532 \mathrm{~nm}$ (visible light) laser illumination. The plasmonic heating drive the thermally driven $\mathrm{CO}_{2}$ reduction reactions under laser illumination. The minimum light intensity that needs to start the $\mathrm{CO}_{2}$ conversion can be achieve using the currently available solar concentrators. The higher robustness of the $\mathrm{Au}-\mathrm{ZnO}$ is very important factor for industrial applications.

The plasmonic heating effect was investigated in detail using the temperature dependent Raman spectroscopy. Temperature programmed reaction experiments, excitation intensity dependent 
catalysis reactions and thermodynamics simulations verified the plasmonic heating assisted $\mathrm{CO}_{2}$ reduction. This method will enable a more efficient method to convert $\mathrm{CO}_{2}$ in to viable chemicals using heterogeneous catalysts with metal nanoparticles and metal oxide under concentrated sun light.

Since it is not worth to reach the melting point of Au by increasing the heat generation it is worth to investigate the efficient heat generation of $\mathrm{Au}-\mathrm{ZnO}$ using the lower light intensity probably natural sunlight. It may possible to achieve this by changing the size, shape and density of the Au nanoparticle. An attempt to increase the heating efficiency of $\mathrm{ZnO}$ by increasing the Au particle density was discussed in next chapter (chapter 6) 


\section{References}

1. D. C. Grills and E. Fujita, The Journal of Physical Chemistry Letters, 2010, 1, 27092718.

2. H. Takeda and O. Ishitani, Coordination Chemistry Reviews, 2010, 254, 346-354.

3. B. Abramović, V. Despotović, D. Šojić and N. Finčur, Reac Kinet Mech Cat, 2014, 1-13.

4. H. Chen, L. Shao, Q. Li and J. Wang, Chemical Society Reviews, 2013, 42, 2679-2724.

5. J.-J. Chen, J. C. S. Wu, P. C. Wu and D. P. Tsai, The Journal of Physical Chemistry C, $2011,115,210-216$.

6. D. B. Ingram and S. Linic, Journal of the American Chemical Society, 2011, 133, 52025205.

7. P. Christopher, H. Xin and S. Linic, Nat Chem, 2011, 3, 467-472.

8. W. H. Hung, M. Aykol, D. Valley, W. Hou and S. B. Cronin, Nano Letters, 2010, 10, 1314-1318.

9. K. L. Kelly, E. Coronado, L. L. Zhao and G. C. Schatz, The Journal of Physical Chemistry B, 2003, 107, 668-677.

10. M. A. El-Sayed, Accounts of Chemical Research, 2001, 34, 257-264.

11. P. Christopher, H. Xin, A. Marimuthu and S. Linic, Nat Mater, 2012, 11, 1044-1050.

12. Y. Tian and T. Tatsuma, Journal of the American Chemical Society, 2005, 127, 76327637.

13. L. Brus, Accounts of Chemical Research, 2008, 41, 1742-1749.

14. W. Hou, W. H. Hung, P. Pavaskar, A. Goeppert, M. Aykol and S. B. Cronin, ACS Catalysis, 2011, 1, 929-936. 
15. M. K. Kumar, S. Krishnamoorthy, L. K. Tan, S. Y. Chiam, S. Tripathy and H. Gao, ACS Catalysis, 2011, 1, 300-308.

16. G. Baffou, C. Girard and R. Quidant, Physical Review Letters, 2010, 104, 136805.

17. M. T. Carlson, A. J. Green and H. H. Richardson, Nano Letters, 2012, 12, 1534-1537.

18. N. Harris, M. J. Ford and M. B. Cortie, The Journal of Physical Chemistry B, 2006, 110, 10701-10707.

19. S. Natesakhawat, J. W. Lekse, J. P. Baltrus, P. R. Ohodnicki, B. H. Howard, X. Deng and C. Matranga, ACS Catalysis, 2012, 2, 1667-1676.

20. M. Behrens, F. Studt, I. Kasatkin, S. Kühl, M. Hävecker, F. Abild-Pedersen, S. Zander, F. Girgsdies, P. Kurr, B.-L. Kniep, M. Tovar, R. W. Fischer, J. K. Nørskov and R. Schlögl, Science, 2012, 336, 893-897.

21. A. O. Govorov, W. Zhang, T. Skeini, H. Richardson, J. Lee and N. A. Kotov, Nanoscale Research Letters, 2006, 1, 84-90.

22. P. R. Ohodnicki, M. P. Buric, T. D. Brown, C. Matranga, C. Wang, J. Baltrus and M. Andio, Nanoscale, 2013, 5, 9030-9039.

23. P. G. Etchegoin, E. C. Le Ru and M. Meyer, The Journal of Chemical Physics, 2006, $125,164705$.

24. A. K. Azad, J. Han and W. Zhang, Applied Physics Letters, 2006, 88, 021103.

25. F. I. Mopsik JOURNAL OF RESEARCH of the National Bureau of Standards - A. Physics and Chemistry 1967, 71A, 287-292.

26. A. O. Govorov and H. H. Richardson, Nano Today, 2007, 2, 30-38.

27. J. W. Li, L. W. Yang, Z. F. Zhou, X. J. Liu, G. F. Xie, Y. Pan and C. Q. Sun, The Journal of Physical Chemistry B, 2010, 114, 1648-1651. 
28. R. Cuscó, E. Alarcón-Lladó, J. Ibáñez, L. Artús, J. Jiménez, B. Wang and M. J. Callahan, Physical Review B, 2007, 75, 165202.

29. C. F. Klingshirn, A. Waag, A. Hoffmann and J. Geurts, Zinc Oxide: From Fundamental Properties Towards Novel Applications, 2010.

30. K. A. Alim, V. A. Fonoberov, M. Shamsa and A. A. Balandin, Journal of Applied Physics, 2005, 97, 124313.

31. H. Kumar Yadav, R. S. Katiyar and V. Gupta, Applied Physics Letters, 2012, 100, 051906.

32. K. Samanta, P. Bhattacharya and R. S. Katiyar, Physical Review B, 2007, 75, 035208.

33. E. Alarcon-Llado, R. Cusco, J. Ibanez, L. Artus, J. Jimenez, B. Wang and M. Callahan, MRS Online Proceedings Library, 2006, 957, null-null.

34. J. Serrano, F. J. Manjón, A. H. Romero, F. Widulle, R. Lauck and M. Cardona, Physical Review Letters, 2003, 90, 055510.

35. J. W. Pomeroy, M. Kuball, H. Lu, W. J. Schaff, X. Wang and A. Yoshikawa, Applied Physics Letters, 2005, 86, 223501.

36. J. Menéndez and M. Cardona, Physical Review B, 1984, 29, 2051-2059.

37. C. Wang, R. L. Thompson, P. Ohodnicki, J. Baltrus and C. Matranga, Journal of Materials Chemistry, 2011, 21, 13452-13457.

38. C. Wang, O. Ranasingha, S. Natesakhawat, P. R. Ohodnicki, M. Andio, J. P. Lewis and C. Matranga, Nanoscale, 2013, 5, 6968-6974. 


\section{Chapter 6}

\section{Synthesis, characterization, and photocatalytic activity of $\underline{\text { Au-ZnO nanopyramids }}$}

\subsection{Introduction}

The previous chapter shows that the plasmonic heating occurs in 5\% Au- $\mathrm{ZnO}$ heterostructure under the $532 \mathrm{~nm} c w$ laser illumination. Also, it was found that the density of $\mathrm{Au}$ nanoparticles affect the final temperature increase at the $\mathrm{ZnO}$ nanoparticles. Therefore, the main objective of this work was increasing the plasmonic heating efficiency by increasing the $\mathrm{Au}$ to $\mathrm{ZnO}$ ratio. However, the synthesis procedure which was used for synthesis of $5 \% \mathrm{Au}-\mathrm{ZnO}$ was not suitable for this. Au- $\mathrm{ZnO}$ nanopyramids were the first choice because every $\mathrm{ZnO}$ nanoparticle has a $\mathrm{Au}$ nanoparticle attached in to the basal surface. Since plasmonic heating is a highly localized process, it was expected to transfer the plasmonincally generated heat from $\mathrm{Au}$ nanoparticle to $\mathrm{ZnO}$ nanoparticle. In 5\% $\mathrm{Au}$ $\mathrm{ZnO}$ sample in previous chapter, all the $\mathrm{ZnO}$ particles do not have attached Au nanoparticle. Au nanoparticle distribution was uneven throughout the $\mathrm{ZnO}$. Therefore plasmonic heating was occurring at certain few hot spots in $5 \% \mathrm{Au}-\mathrm{ZnO}$ sample (Figure 6.1). But when consider the large area in micrometer size for laser illumination, it is difficult to identify these hot spots separately. Since Au-ZnO nanopyramids has at least one Au nanoparticle attached in to the $\mathrm{ZnO}$, every $\mathrm{ZnO}$ nanoparticle can be considered as a hot spot for plasmonic heating (Figure 6.2). The examples for hot spots can be found inside the red circles in Figures 6.1 and 6.2. 
However, due to several possible reasons $\mathrm{Au}-\mathrm{ZnO}$ nanopyramids didn't show any plasmonic heating activity under the same laser intensities as $5 \% \mathrm{Au}-\mathrm{ZnO}$ heterostructures. But $\mathrm{Au}-\mathrm{ZnO}$ showed excellent photocatalytic activity for organic dye degradation reactions with the band gap excitation of $\mathrm{ZnO}$.

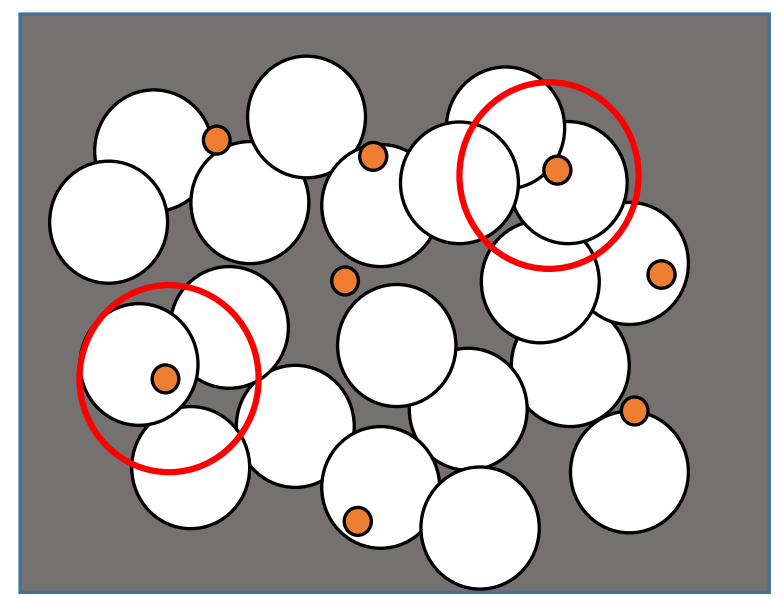

Figure 6.1 Schematic diagram of $5 \% \mathrm{Au}-\mathrm{ZnO}$

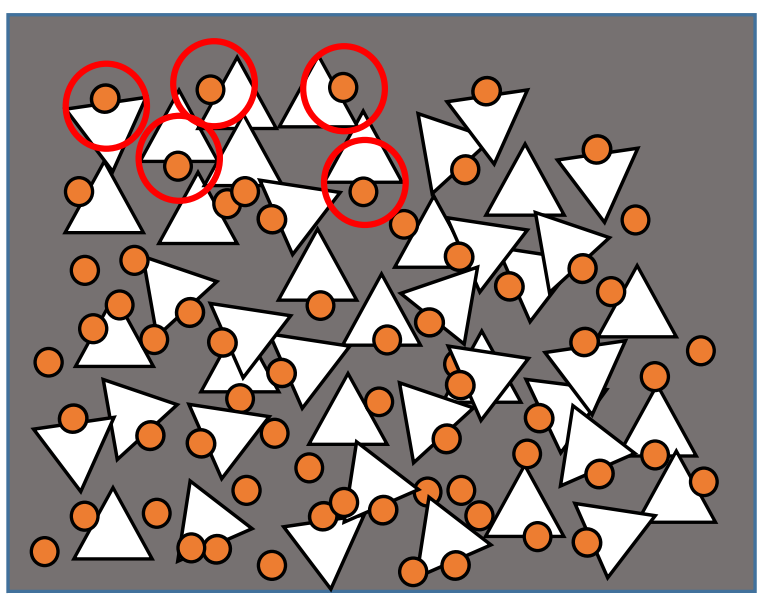

Figure 6.2 Schematic diagram of $\mathrm{Au}-\mathrm{ZnO}$ nanopyramids

Several papers in the literature describe how the size and shape of $\mathrm{Au}-\mathrm{ZnO}$ heterostructures can be controlled by using different synthetic approaches. ${ }^{1-3}$ Yao et al. ${ }^{3}$ were able to selectively photo-deposit $\mathrm{Au}$ particles at the tips of $\mathrm{ZnO}$ nanoparticles with a pyramid shape. Li et al. ${ }^{2}$ and Flomin et al. ${ }^{1}$ used wet chemical methods to grow well-formed $\mathrm{ZnO}$ nanopyramids from the surfaces of Au seeds and evaluated the photocatalytic activity of these heterostructures. He et al. ${ }^{4}$ have reported on heterogeneous powders of $\mathrm{Au}-\mathrm{ZnO}$ heterostructures which have been evaluated for the photodegradation of bacteria.

All of these works find that very subtle changes in the synthetic approaches yield dramatic changes in the general morphology of $\mathrm{Au}-\mathrm{ZnO}$ particles. In particular, the synthetic steps 
can be used to control where the Au is deposited as well as the structural details of the Au$\mathrm{ZnO}$ interface. Currently, there is little understanding in the literature of how these subtle structural details impact localized changes in electronic structure near the interface, defects arising from the heterostructuring step, and the final photocatalytic activity of the heterostructure. As such, more investigations are needed to develop insight into the final chemical properties of these materials.

In this work, $\mathrm{Au}-\mathrm{ZnO}$ heterostructures were synthesized using a wet chemical method that attempts to minimize the use of organic surfactants that can block catalytically active surface sites. In this system, Au nanoparticle seeds are used to nucleate the growth of $\mathrm{ZnO}$ structures with a pyramidal-type structure. A previously unreported lattice expansion of $\sim 3.5 \%$ is found for $\mathrm{ZnO}$ in the first $\sim 1.5 \mathrm{~nm}$ of the $\mathrm{Au}-\mathrm{ZnO}$ interface. Photocatalytic dye degradation studies of $\mathrm{Au}-\mathrm{ZnO}$ find that direct gap excitation is required to initiate photodecomposition and that the heterostructure has significantly higher catalytic activity than pure $\mathrm{ZnO}$. This work provides experimental evidence that the $\mathrm{Au}$ nanoparticles contribute to the higher catalytic activity of $\mathrm{Au}-\mathrm{ZnO}$ by widening the band gap of $\mathrm{ZnO}$, improving band alignment for the injection of charge carriers into solution species, and by increasing spatial separation of photogenerated charge carriers. 


\subsection{Materials preparation}

Hydrogen tetrachloroaurate (III) hydrate $\left(\mathrm{HAuCl}_{4} \bullet 4 \mathrm{H}_{2} \mathrm{O}\right), 99.9 \%$, Zinc acetate dihydrate, methanol, $99.9 \%$, hexane, $99.9 \%$ and acetone, $99.9 \%$ were purchased from Alfa Aesar. Tert-butylamine borane complex, oleylamine, C18 content 80-90\% and dodecanol, $98 \%$ were purchased from Acros Organics.

\subsubsection{Synthesis of Au nanoparticles}

Au nanoparticles were synthesized using the procedure of Peng et al. ${ }^{5} \mathrm{HAuCl}_{4} \bullet 4 \mathrm{H}_{2} \mathrm{O}(0.5$ mmol) was added to a three-neck flask with the mixture of oleylamine $(2 \mathrm{ml})$ and hexane (20 ml) at room temperature. This precursor mixture was purged under flowing $\mathrm{N}_{2}$ for $\sim 10$ minutes. Tert-butylamine borane complex $(1 \mathrm{mmol})$ was dissolved in oleylamine $(2 \mathrm{ml})$ and hexane $(2 \mathrm{ml})$ in a glove box. Then, this reducing solution was quickly injected to the precursor solution in the three-neck flask. The reduction reaction started instantaneously and the color of the precursor solution changed to a deep purple within a few seconds. This solution was stirred for 1 hour at room temperature. Ethanol was then added to the colloidal solution in order to precipitate the Au nanoparticles followed by centrifugation for 20 minutes at $3000 \mathrm{rpm}$ to collect the $\mathrm{Au}$ nanoparticles. $60 \mathrm{mg}$ of precipitate $(\mathrm{Au}$ nanoparticles) were re-dispersed in $5 \mathrm{ml}$ of hexane. The primary modification of Peng's recipe involves replacing tetralin with hexane, since tetralin was difficult to fully rinse from the sample and seemed to block surface access during photocatalytic studies. 


\subsubsection{Synthesis of Au-ZnO nanopyramids}

Synthesis of $\mathrm{Au}-\mathrm{ZnO}$ heterostructures was completed using a previously reported recipe which had been modified to reduce the use of heavier organic solvents that tend to leave surface residue on the samples. ${ }^{2}$ Alsmost all the parameters in the syntheis procedure is critical to get a high quality $\mathrm{ZnO}$ nanoparticle. First, it is important to find the corect amount of gold and zinc precursors. If there is excess amount of zinc precusor, it is posible to have free $\mathrm{ZnO}$ nanoparticles without $\mathrm{Au}$ seeds which is against the main objective. If there is excess amount of gold precursors, it is possible to have free Au nanopartcles in the Au$\mathrm{ZnO}$ sample which might be a problem at least by adding some volume and weight to the catalyst. The organic solvents act a critical role for mainting the correct shape and size of the $\mathrm{ZnO}$ nanoparticles. Li et $a l^{6}$ reported that synthesis of different shapes (nanopyramids, bullet like nanocrystals, pencil like nanorods) can be achieved by changing the amount of organic solvents. Also, synthesis temperature and reaction time affects the number of incomplete $\mathrm{ZnO}$ nanoparticles and quality of the facets. ${ }^{6}$ The shape, size and quality affect the photocatalytic perfomance. Also, remaining organic solvents in the sample after the syntheis may reduce the photocatalytic performance by blocking the active sites. In other words, the amount of these parameters in the synthesis procedure are competing each other with respect to catalytic performance. Therefore it is important to optimize each parameter one by one to achive the best $\mathrm{Au}-\mathrm{ZnO}$ nanopyramids. Several attempts were made in order to optimize the recipe to get high quality $\mathrm{Au}-\mathrm{ZnO}$ nanopyramids with lower levels organic molecules in the synthesis steps (Table 6.1). In most of these samples, $\mathrm{Au}-\mathrm{ZnO}$ heterostrucutres were not observed. For the first time,sample 26 (Table 6.1) resulted high quality $\mathrm{Au}-\mathrm{ZnO}$ heterostrucutred nanopyramids. Sample 26 shows pyramid shape $\mathrm{ZnO}$ 
nanoparticles with attached $\mathrm{Au}$ nanoparticles at the basel surface. Also free $\mathrm{ZnO}$ nanoparticles were not observed that much. Again, a few attempts were made to further reduce the organic molecules while mainting the quality of $\mathrm{Au}-\mathrm{ZnO}$ heterostrucutres in order to minimize the active site blocking by heavy organic molecules. Since sample 31 has similar quality of $\mathrm{Au}-\mathrm{ZnO}$ nanopyramids with less amount of organic solvents compared to sample 26, it was chosen the as the optimized recipe. Figures 6.3- 6.10 show the Transmission Electron Microscope images of sample 21- 31 respectively.

Zinc acetate dihydrate $(275 \mathrm{mg})$ was added to a mixture of oleylamine $(1.5 \mathrm{ml})$ and dodecanol $(6 \mathrm{ml})$ in a three-neck flask which was then purged for 10 minutes under $\mathrm{N}_{2}$ flow. After that, pre-prepared Au seeds (60 mg dispersed in $5 \mathrm{ml}$ hexane) were quickly injected into the flask and the temperature was slowly increased to and held at $140{ }^{\circ} \mathrm{C}$ for 90 minuntes. The sample was then allowed to cool to room temperature. The cooled solution was rinsed with an excess of methanol several times, centrifuged, and then rinsed with acetone, centrifuged, and dispersed in hexane. After removing the liquid phase, acetone was added to the test tubes and centrifuged for 10 minutes. The precipitate was dissolved in a small amount of hexane and combined to one test tube. Then, methanol was added to the solution and centrifuged for 10 minutes. This last step was repeated one more time. The precipitate was dried under $\mathrm{N}_{2}$ flow for few minutes. At this stage, precipitate was no longer soluble in hexane due to the lack of organic capping molecules for solubility. Then, the sample was further cleaned with oxygen plasma for 60 minutes to remove any excess organic molecules. 
Table 6.1 Au-ZnO nanopyramids synthesis parameters

\begin{tabular}{|c|c|c|c|c|c|c|}
\hline Sample & $\begin{array}{c}\text { Au } \\
\text { precursor } \\
(\mathbf{m g})\end{array}$ & $\begin{array}{c}\text { Zinc } \\
\text { precursor } \\
(\mathbf{m g})\end{array}$ & $\begin{array}{c}\text { Oleylamine } \\
(\mathbf{m l})\end{array}$ & $\begin{array}{c}\text { Dodecanol } \\
(\mathbf{m l})\end{array}$ & $\begin{array}{c}\text { Reaction } \\
\text { temperature } \\
\left({ }^{\mathbf{C}} \mathbf{C}\right)\end{array}$ & $\begin{array}{c}\text { Reaction } \\
\text { time } \\
(\mathbf{m i n u t e s})\end{array}$ \\
\hline 1 & 60 & 92 & 3 & 6 & 180 & 5 \\
\hline 2 & 6 & 92 & 3 & 6 & 180 & 5 \\
\hline 3 & 12 & 92 & 3 & 6 & 180 & 5 \\
\hline 4 & 24 & 92 & 3 & 6 & 180 & 5 \\
\hline 5 & 36 & 92 & 3 & 6 & 180 & 5 \\
\hline 6 & 48 & 92 & 3 & 6 & 180 & 5 \\
\hline 7 & 60 & 92 & 3 & 6 & 140 & 5 \\
\hline 8 & 60 & 92 & 3 & 6 & 220 & 5 \\
\hline 9 & 60 & 92 & 3 & 6 & 180 & 60 \\
\hline 10 & 60 & 92 & 3 & 6 & 180 & 30 \\
\hline 11 & 60 & 920 & 3 & 6 & 180 & 30 \\
\hline 12 & 60 & 184 & 3 & 6 & 120 & 60 \\
\hline 13 & 60 & 368 & 3 & 6 & 120 & 60 \\
\hline 14 & 60 & 92 & 3 & 6 & 160 & 30 \\
\hline 15 & 60 & 184 & 3 & 6 & 160 & 30 \\
\hline 16 & 60 & 184 & 3 & 6 & 100 & 60 \\
\hline 17 & 60 & 368 & 3 & 6 & 100 & 60 \\
\hline 18 & 60 & 92 & 3 & 6 & 140 & 30 \\
\hline 19 & 60 & 184 & 3 & 6 & 140 & 30 \\
\hline 20 & 60 & 184 & 3 & 6 & 140 & 90 \\
\hline 21 & 60 & 184 & 3 & 6 & 140 & 30 \\
\hline 22 & 60 & 275 & 3 & 6 & 140 & 30 \\
\hline 23 & 60 & 275 & 3 & 6 & 140 & 180 \\
\hline 24 & 60 & 368 & 3 & 6 & 140 & 180 \\
\hline 25 & 60 & 275 & 3 & 6 & 140 & 120 \\
\hline
\end{tabular}




\begin{tabular}{|c|c|c|c|c|c|c|}
\hline $\mathbf{2 6}$ & $\mathbf{6 0}$ & $\mathbf{2 7 5}$ & $\mathbf{3}$ & $\mathbf{6}$ & $\mathbf{1 4 0}$ & $\mathbf{9 0}$ \\
\hline 27 & 3 & 275 & 1.5 & 6 & 140 & 90 \\
\hline 28 & 6 & 275 & 0.5 & 3 & 140 & 90 \\
\hline 29 & 12 & 275 & 0.5 & 3 & 140 & 90 \\
\hline 30 & 60 & 275 & 0.5 & 3 & 140 & 90 \\
\hline $\mathbf{3 1}$ & $\mathbf{6 0}$ & $\mathbf{2 7 5}$ & $\mathbf{1 . 5}$ & $\mathbf{6}$ & $\mathbf{1 4 0}$ & $\mathbf{9 0}$ \\
\hline
\end{tabular}

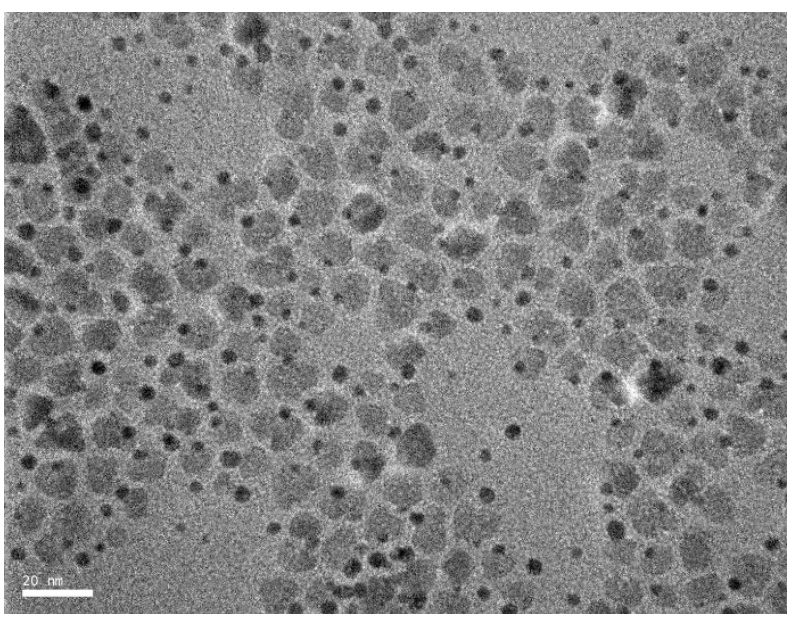

Figure 6.3 TEM image of $\mathrm{Au}-\mathrm{ZnO}$ (sample 21)

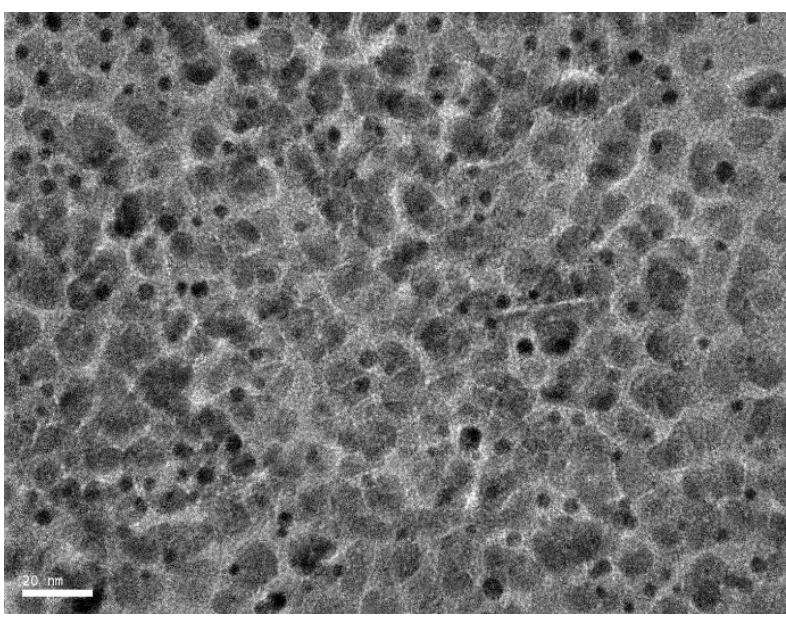

Figure 6.5 TEM image of $\mathrm{Au}-\mathrm{ZnO}$ (sample 23)

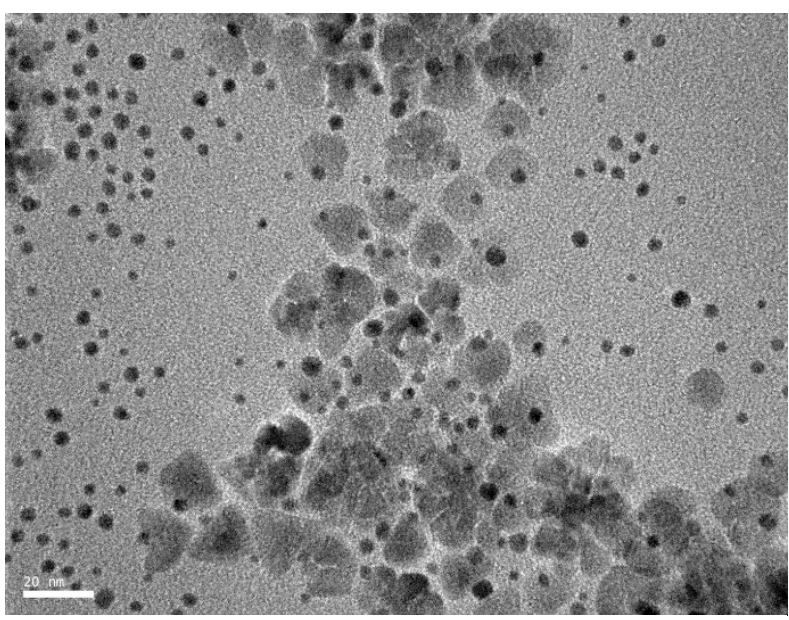

Figure 6.4 TEM image of $\mathrm{Au}-\mathrm{ZnO}$ (sample 22)

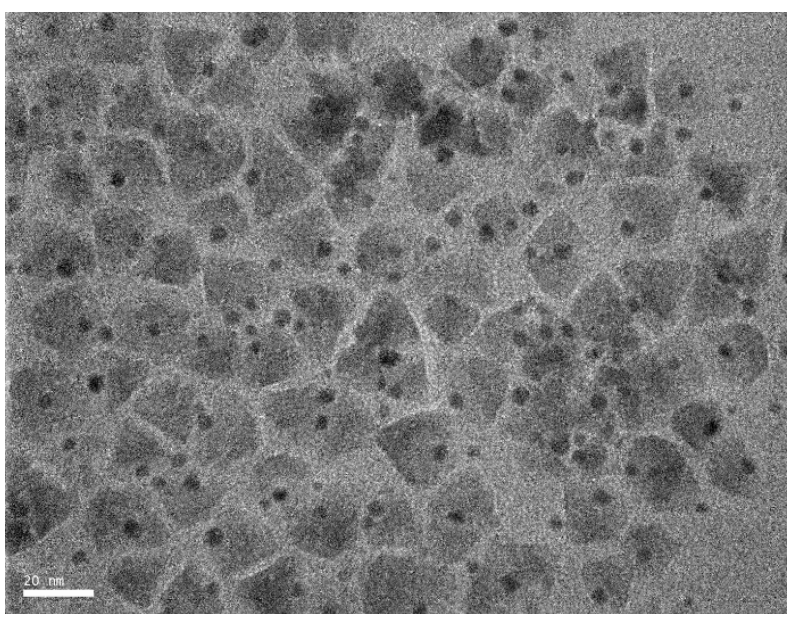

Figure 6.6 TEM image of $\mathrm{Au}-\mathrm{ZnO}$ (sample 26) 


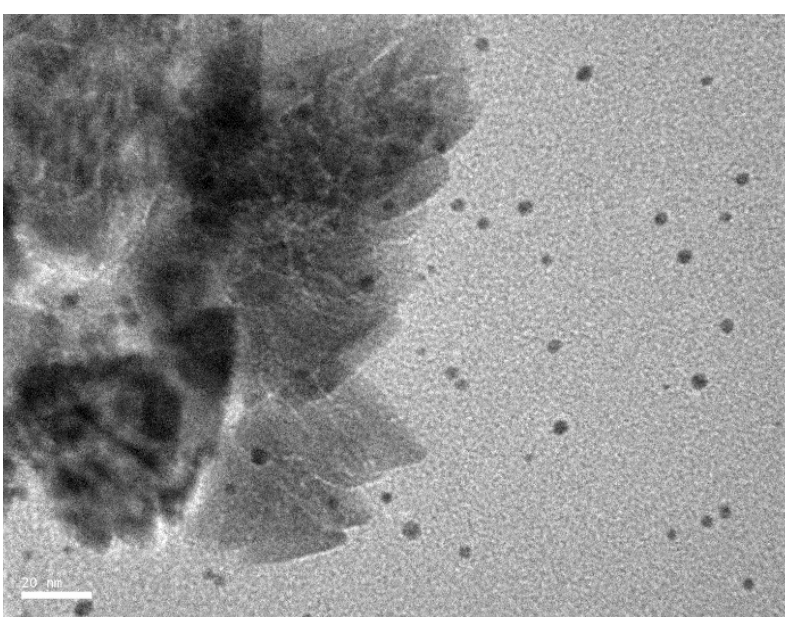

Figure 6.7 TEM image of Au-ZnO (sample 27)

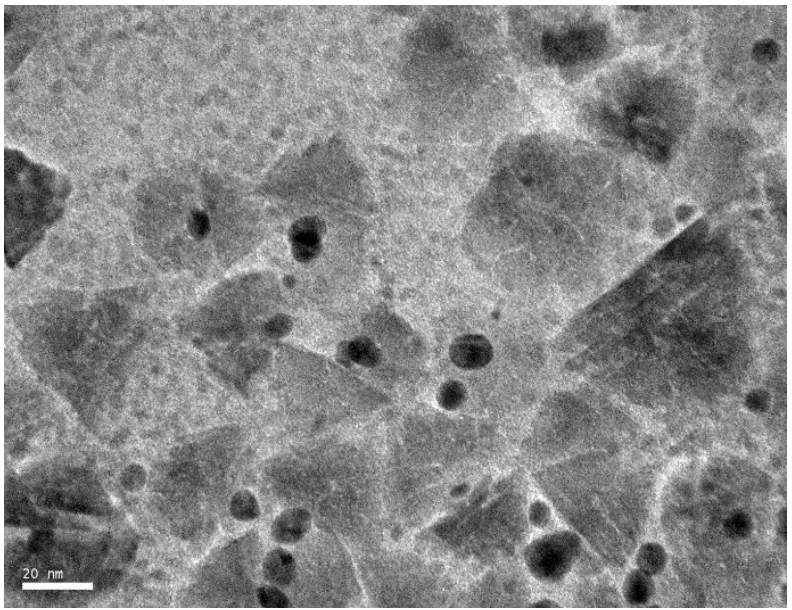

Figure 6.9 TEM image of $\mathrm{Au}-\mathrm{ZnO}$ (sample 30)

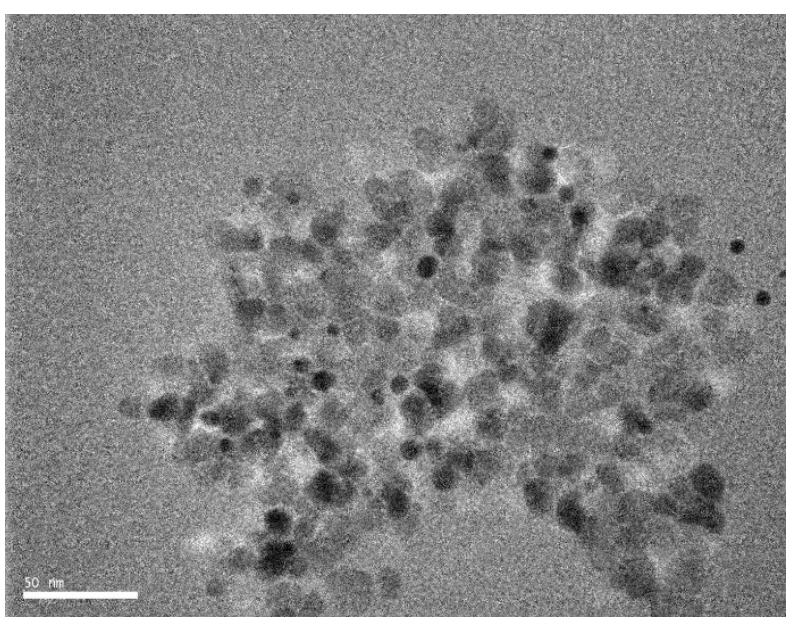

Figure 6.8 TEM image of Au-ZnO (sample 29)

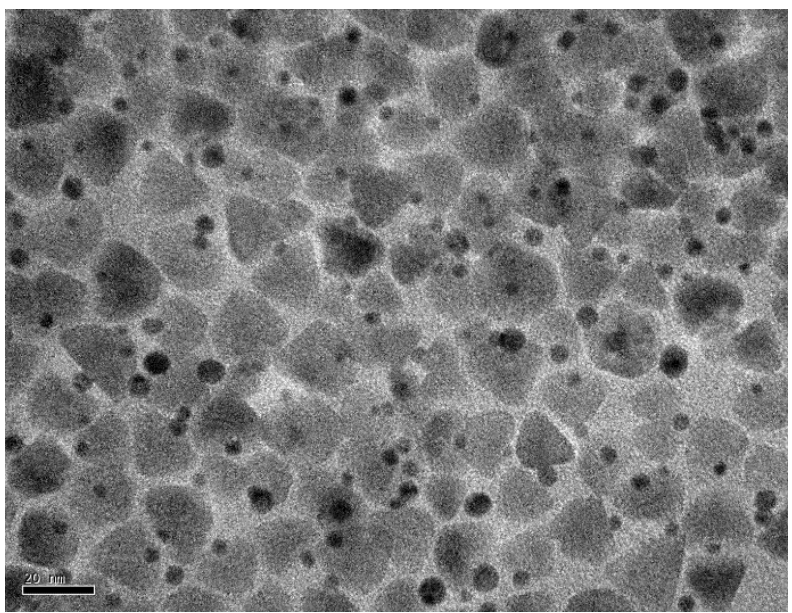

Figure 6.10 TEM image of Au-ZnO (sample 31)

\subsubsection{Synthesis of pure $\mathrm{ZnO}$ and $\mathrm{Au}-\mathrm{SiO}_{2}$}

The pure $\mathrm{ZnO}$ nanoparticles were synthesized using the exact same procedure described above for the $\mathrm{Au}-\mathrm{ZnO}$ heterostructures, except the $\mathrm{Au}$ seeds were omitted. $\mathrm{Au}-\mathrm{SiO}_{2}$ was prepared as a control catalyst by mixing $60 \mathrm{mg}$ of Au seeds with $275 \mathrm{mg}$ of commercial $\mathrm{SiO}_{2}$. 


\subsection{Methylene blue degradation reactions}

Prior to dye degradation runs the catalysts were treated with an $\mathrm{O}_{2}$ plasma for $\sim 60$ mintues to remove surface-bound organics from the synthesis steps that could interfere with surface adsoprtion and photocatlytic activity. The FTIR spectra before and after the plasma etching for pure $\mathrm{ZnO}$ and $\mathrm{Au}-\mathrm{ZnO}$, respectively, illustrate the complete removal of organics (Figure 6.11 and 6.12).

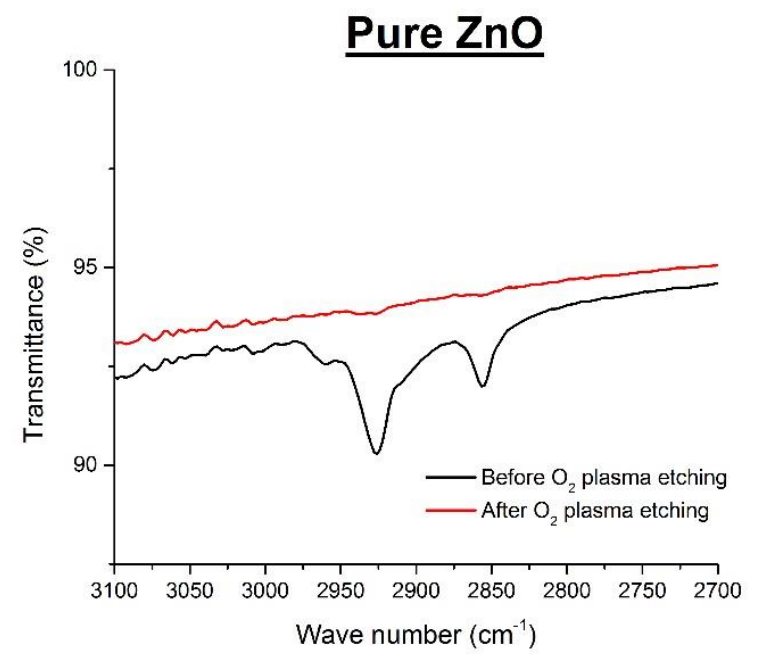

Figure 6.11 FTIR spectra of pure $\mathrm{ZnO}$ before (black) and after (red) the plasma etching

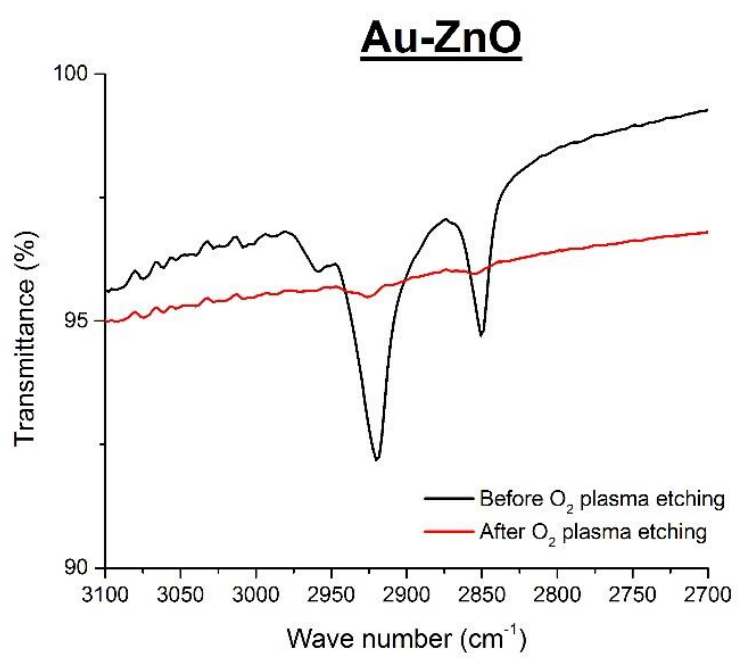

Figure 6.12 FTIR spectra of $\mathrm{Au}-\mathrm{ZnO}$ before (black) and after (red) the plasma etching

Methylene blue (MB) dye photodegradation was used as a model reaction in order to investigate the catalytic performance of $\mathrm{Au}-\mathrm{ZnO}$. The catalyst (27 $\mathrm{mg}$ ) was placed into 10 $\mathrm{ml}$ of $20 \mu \mathrm{mol} / \mathrm{L} \mathrm{MB}$ and soaked overnight ( 15 hours) in the dark to stabilize the surface adsorption and desorption equilibrium of the dye. A Newport $300 \mathrm{~W}$ Xe arc lamp with a manual shutter was used to illuminate the sample. Agilent $8453 \mathrm{UV} / \mathrm{Vis} / \mathrm{NIR}$ spectrophotometer was used to collect the absorption spectra during the MB degradation 
reactions. Edmunds TECHSPEC longpass filters were used for wavelength dependent experiments. In photocatalysis studies, the sample mixture above was exposed to light for a predetermined period of time. Then $6 \mathrm{ml}$ of the reaction solution was centrifuged to separate the solid catalyst from solution. The optical spectrum of the centrifuged solution was then taken. Absorption spectra were recorded every 10 minutes for a total of $\sim 80$ minutes. The spectral area under the absorption peak around $664 \mathrm{~nm}$ which is the characteristic peak for $\mathrm{MB}$ was calculated after the correction of baseline shift and background subtraction to get an accurate estimation for MB concentration.

\subsection{Results and Discussion}

\subsubsection{Characterization of nanoparticle samples using UV-Vis absorption spectra}

Figure 6.13 shows the UV-Vis absorption spectra for the Au seeds, pure $\mathrm{ZnO}$, and $\mathrm{Au}-\mathrm{ZnO}$ heterostructures used in this study. The broad absorption peak around $510 \mathrm{~nm}$ for the $\mathrm{Au}$ seeds is due to the plasmon resonance of these nanoparticles. After growth of the $\mathrm{ZnO}$ on the seeds, this plasmon resonance red-shifts and narrows due to Ostwald ripening of the Au nanoparticles which occurs at the temperatures used for the growth of $\mathrm{ZnO}^{2}$ The peak maximum for the Au plasmon resonance in $\mathrm{Au}-\mathrm{ZnO}$ was observed around $522 \mathrm{~nm}$. The changing dielectric environment caused by the newly formed $\mathrm{Au}-\mathrm{ZnO}$ interface is an additional physical mechanism that likely contributes to the red-shifting of the plasmon feature. ${ }^{7,8}$ To confirm this mechanism we have conducted a simple model calculation (Figure 6.14) using the quasi-static approximation ${ }^{9}$ to estimate the plasmon peak positions and expected shift due to differences in the dielectric environment. The calculations was 
done according following an example shown in Ohodnicki et al. ${ }^{9}$ (see the section 5.2 for detail calculation procedure)

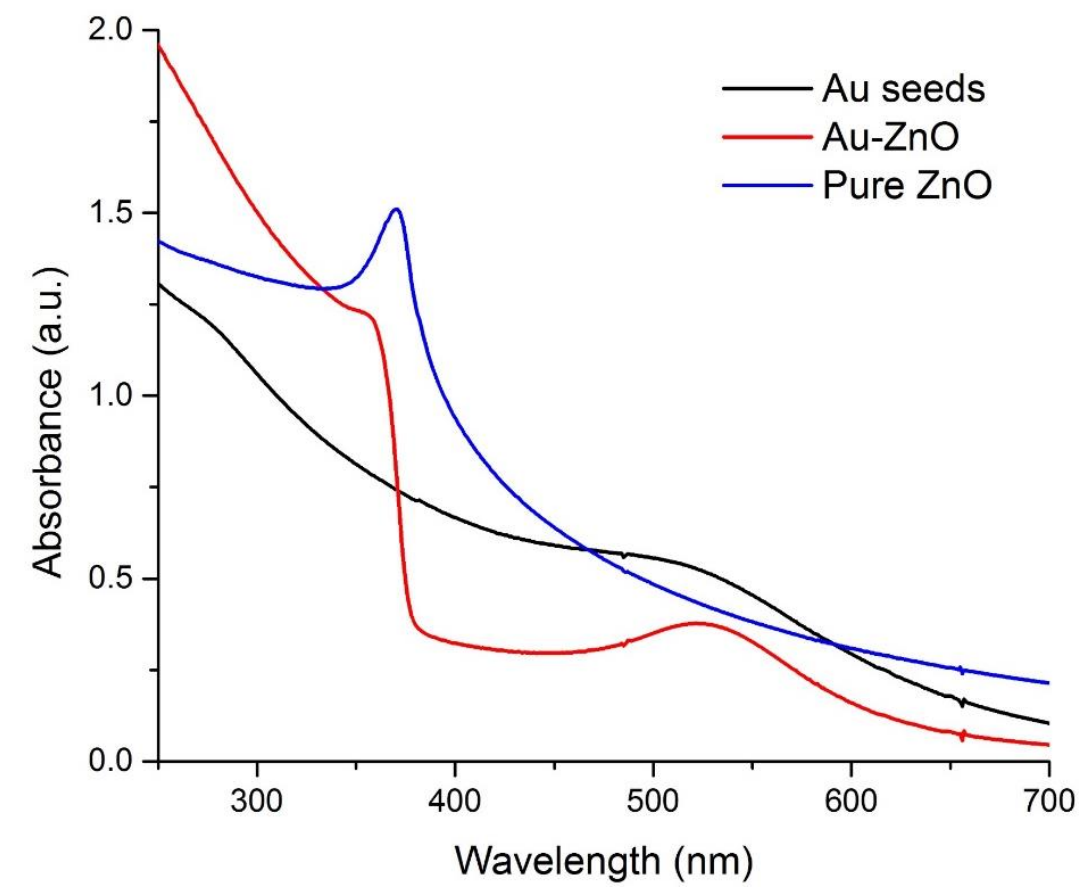

Figure 6.13 UV-Vis absorption spectra for Au seeds (black), pure $\mathrm{ZnO}$ (blue) and $\mathrm{Au}-\mathrm{ZnO}$ heterostructures (red).

Our model calculation predicts that $5 \mathrm{~nm}$ Au seeds in hexane have a plasmon resonance at $\sim 504 \mathrm{~nm}$ that shifts by $\sim 48 \mathrm{~nm}$ when fully encapsulated in $\mathrm{ZnO}$. Considering that our $\mathrm{Au}$ particles are slightly larger than $5 \mathrm{~nm}$, have a distribution in sizes, and are not fully encapsulated by $\mathrm{ZnO}$, the resonance features and red-shift noted in Figure 6.13 are reasonable and qualitatively consistent with a shift due to dielectric changes. Additionally, Khan et $a l^{10}$ have attributed the red-shift noted above, in part, to electron density changes which occur when charge flows from $\mathrm{Ag}$ to $\mathrm{ZnO}$ as Fermi levels equilibrate. A similar electron density change in $\mathrm{Au}-\mathrm{ZnO}$ would also cause red-shifting of the plasmon resonance, 
although quantifying this effect relative to the spectral changes mentioned above is difficult.

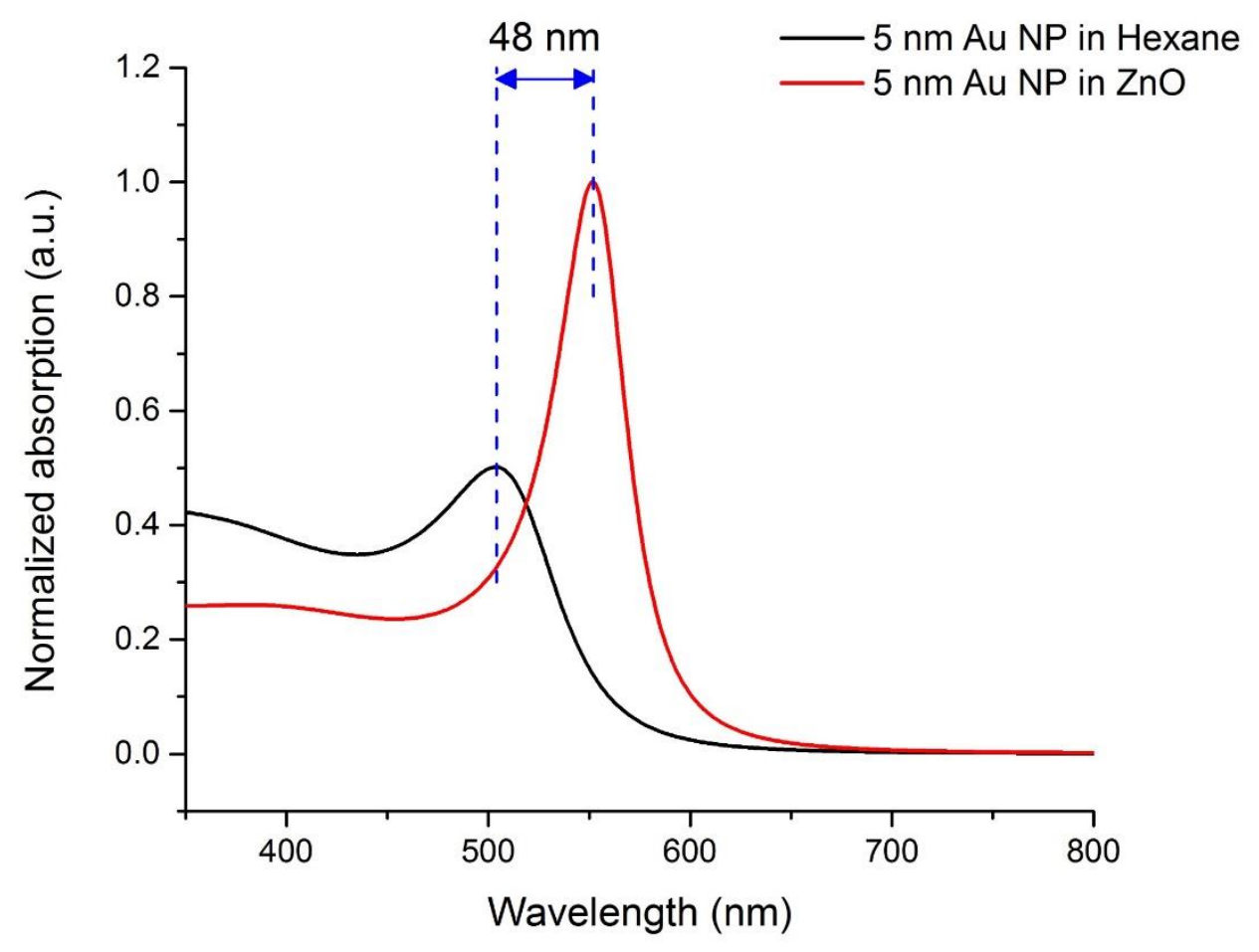

Figure 6.14 Comparison of Au nanoparticle suspended in hexane and full embedded in $\mathrm{ZnO}$

Figure 6.15 shows the Tauc plots of the absorbance spectra to estimate the direct and indirect band gaps of $\mathrm{Au}-\mathrm{ZnO}$ and $\mathrm{ZnO}$. Tauc plots estimate the direct band gap of $\mathrm{ZnO}$ at $405 \mathrm{~nm}(3.06 \mathrm{eV})$ in the pure $\mathrm{ZnO}$ sample and $\sim 383 \mathrm{~nm}(3.23 \mathrm{eV})$ in the $\mathrm{Au}-\mathrm{ZnO}$ sample. There is a strong scattering contribution to the pure $\mathrm{ZnO}$ spectra that can complicate band gap estimates; however, the peak spectral features associated with the band edge for the $\mathrm{Au}-\mathrm{ZnO}$ and $\mathrm{ZnO}$ samples are also shifted from each other. While the mechanism responsible for this bandgap shift is not fully understood, 
He et al. ${ }^{4}$ have also reported a Au-loading dependent bandgap in $\mathrm{Au}-\mathrm{ZnO}$ heterostructures formed by the reduction of $\mathrm{HAuCl}_{4}$ on pre-formed $\mathrm{ZnO}$ nanoparticles.
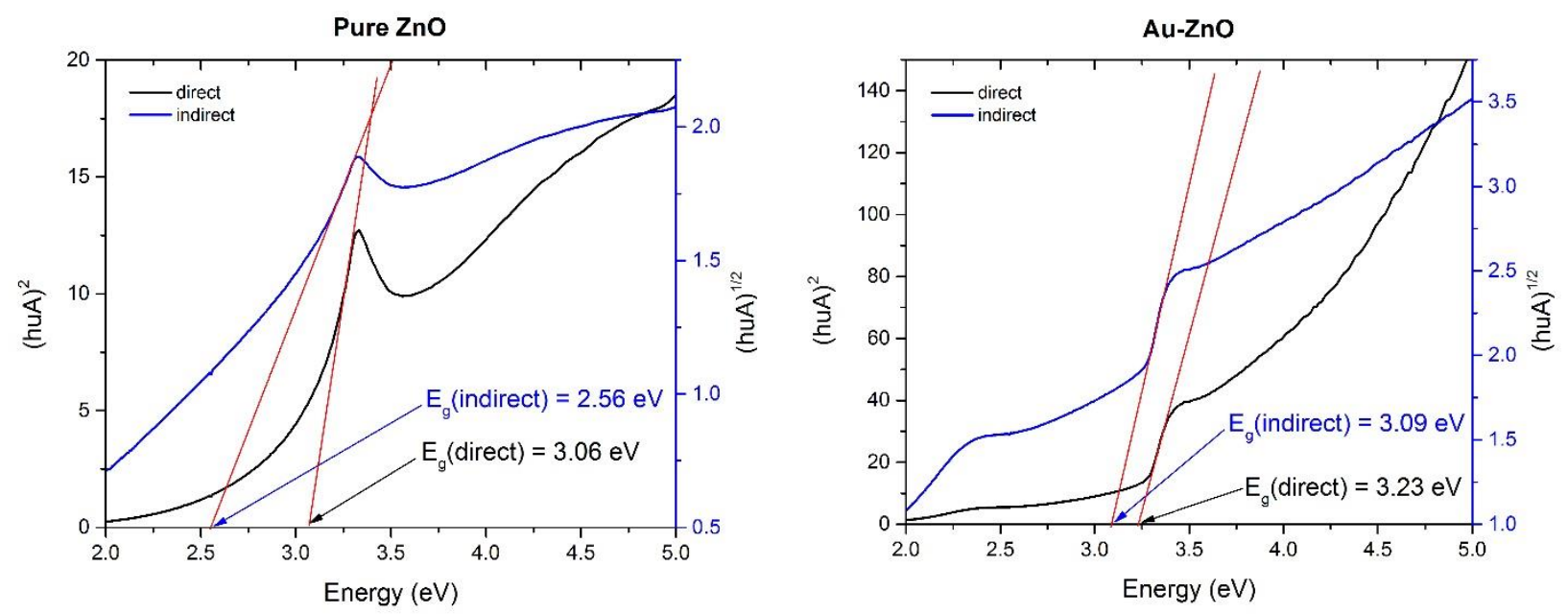

Figure 6.15 Tauc plots for the direct (black) and indirect (blue) band gap estimations of pure $\mathrm{ZnO}$ and $\mathrm{Au}-\mathrm{ZnO}$

\subsubsection{Characterization of TEM and HRTEM images}

Pure $\mathrm{ZnO}$ was synthesized without the use of $\mathrm{Au}$ seeds. In these samples the $\mathrm{ZnO}$ has particle sizes of $\sim 20-30 \mathrm{~nm}$ (Figure 6.16). The average size of the Au seeds used to nucleate the growth of $\mathrm{ZnO}$ is between 4-6 nm (Figure 6.17). Representative TEM images of the $\mathrm{Au}-\mathrm{ZnO}$ heterostructures find Au sizes between 5-7 nm (Figure 6.18). The increase in $\mathrm{Au}$ particle size in the heterostructure is consistent with the red-shifted plasmon feature noted in Figure 6.13. Au nanoparticles that are unattached to $\mathrm{ZnO}$ can also be seen in Figure 6.18 with diameters of $\sim 8-9 \mathrm{~nm}$. These large Au nanoparticles also likely contribute to the redshifting of the Au plasmon resonance peak. As a result of the free Au seeds, the photocatalysis experiments described below also evaluated similar $\mathrm{Au}$ nanoparticles deposited on an inert $\mathrm{SiO}_{2}$ in order to assess their contribution (or lack of) to the photodegradation of methylene blue (see photocatalysis discussion in section 6.4.4). 
The TEM images in Figure 6.18 show that the $\mathrm{ZnO}$ particle sizes in the heterostructures are around 18-22 nm. Additional TEM and HRTEM images (Figures 6.19 and 6.20) illustrate the $\mathrm{ZnO}$ is single-crystalline and possesses a pyramidal-type morphology with a hexagonally shaped base. A similar nanopyramid shape has been reported previously for the $\mathrm{ZnO}$ component of $\mathrm{Au}-\mathrm{ZnO}$ heterostructures, which are grown using a similar synthetic procedure as we report here. ${ }^{2}$ Likewise, similar nanopyramids resulted from a one-pot strategy capable of depositing the Au either at the base or tips of the pyramids. ${ }^{1}$ This type of nanopyramidal $\mathrm{ZnO}$ was also reported in a study that synthesized the $\mathrm{ZnO}$ structures first and later photodeposited Au particles at the tips. ${ }^{3}$ The more heterogeneous nature of the nanopyramids in this study can be attributed to the lower levels of surfactants employed for both the $\mathrm{Au}$ seed and $\mathrm{ZnO}$ synthesis. Since organic contamination can interfere with subsequent catalysis studies by blocking the access to surface sites, their usage was minimized as much as possible.

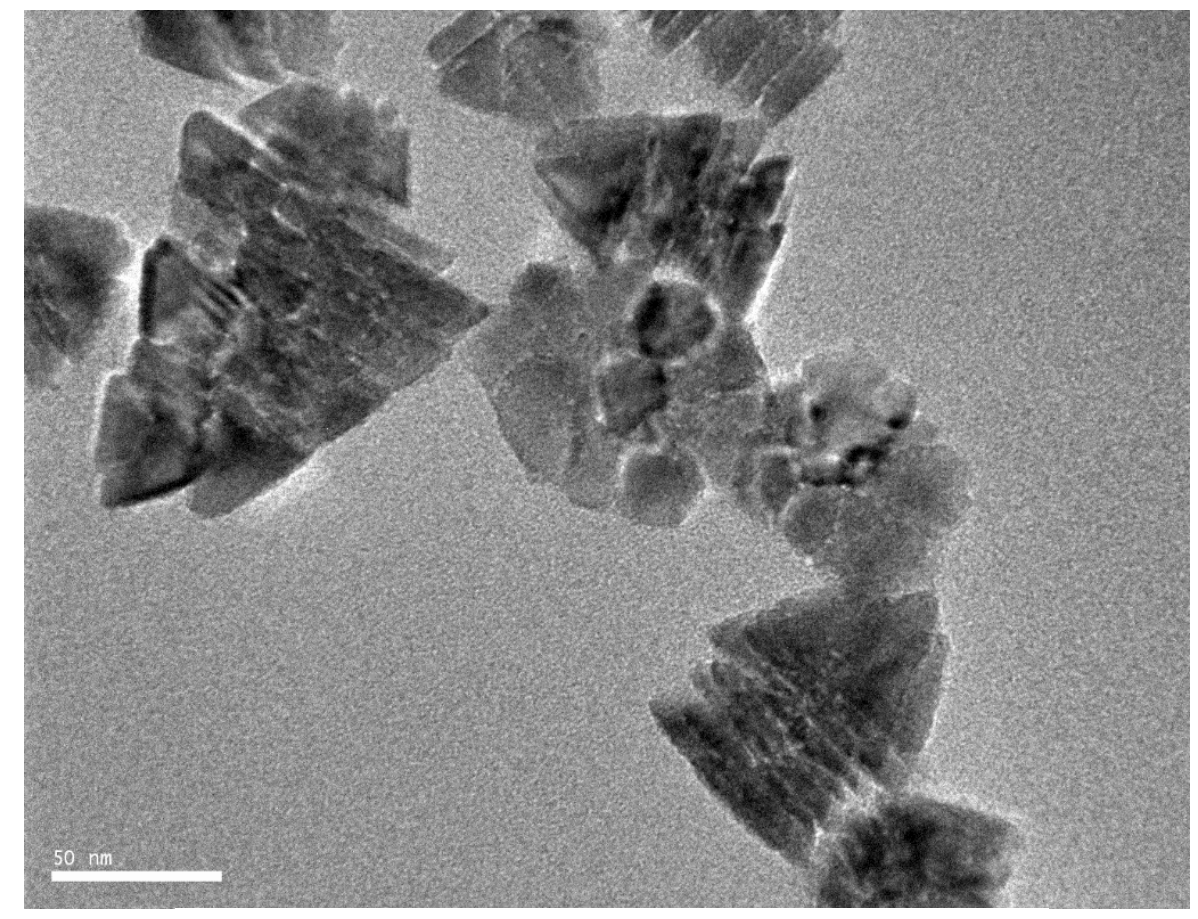

Figure 6.16 TEM images of pure $\mathrm{ZnO}$ nanopyramids 


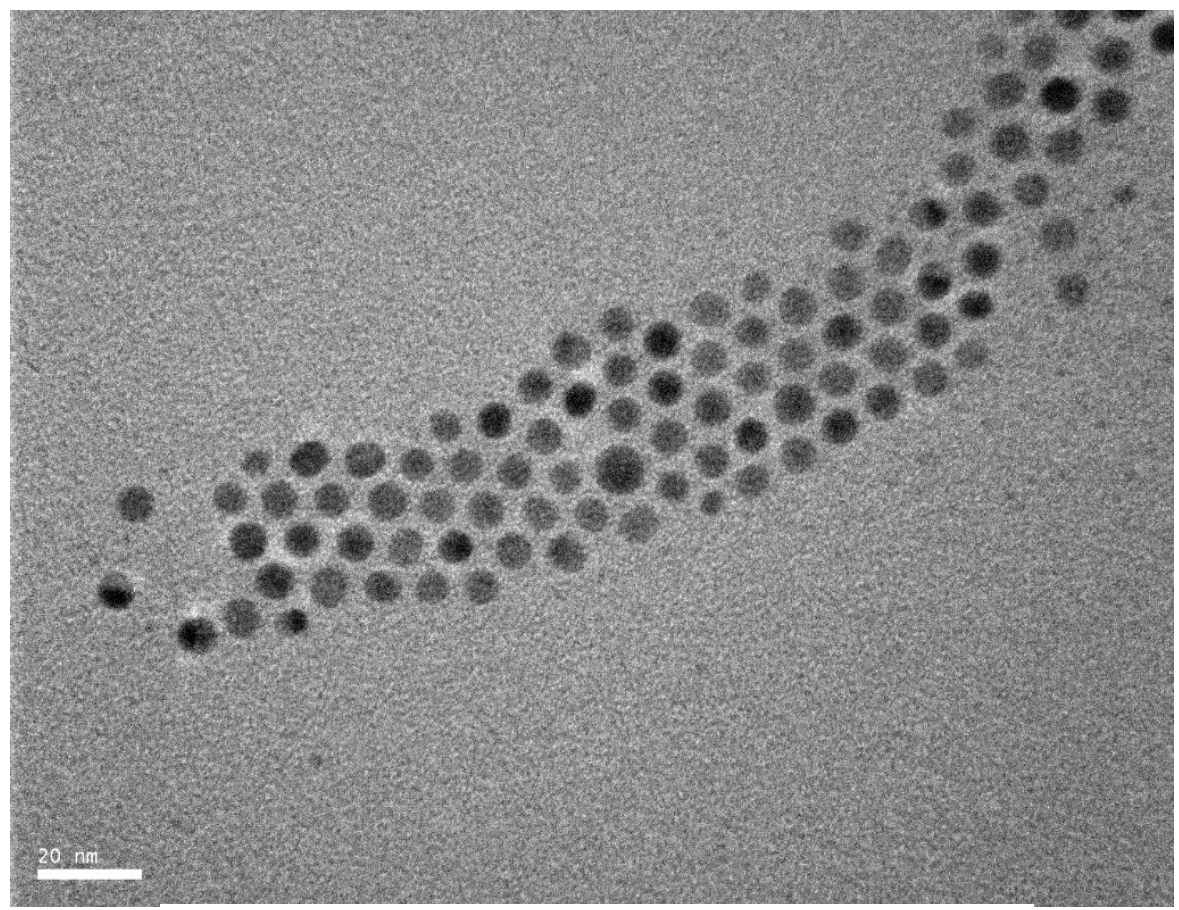

Figure 6.17 TEM images of Au seeds

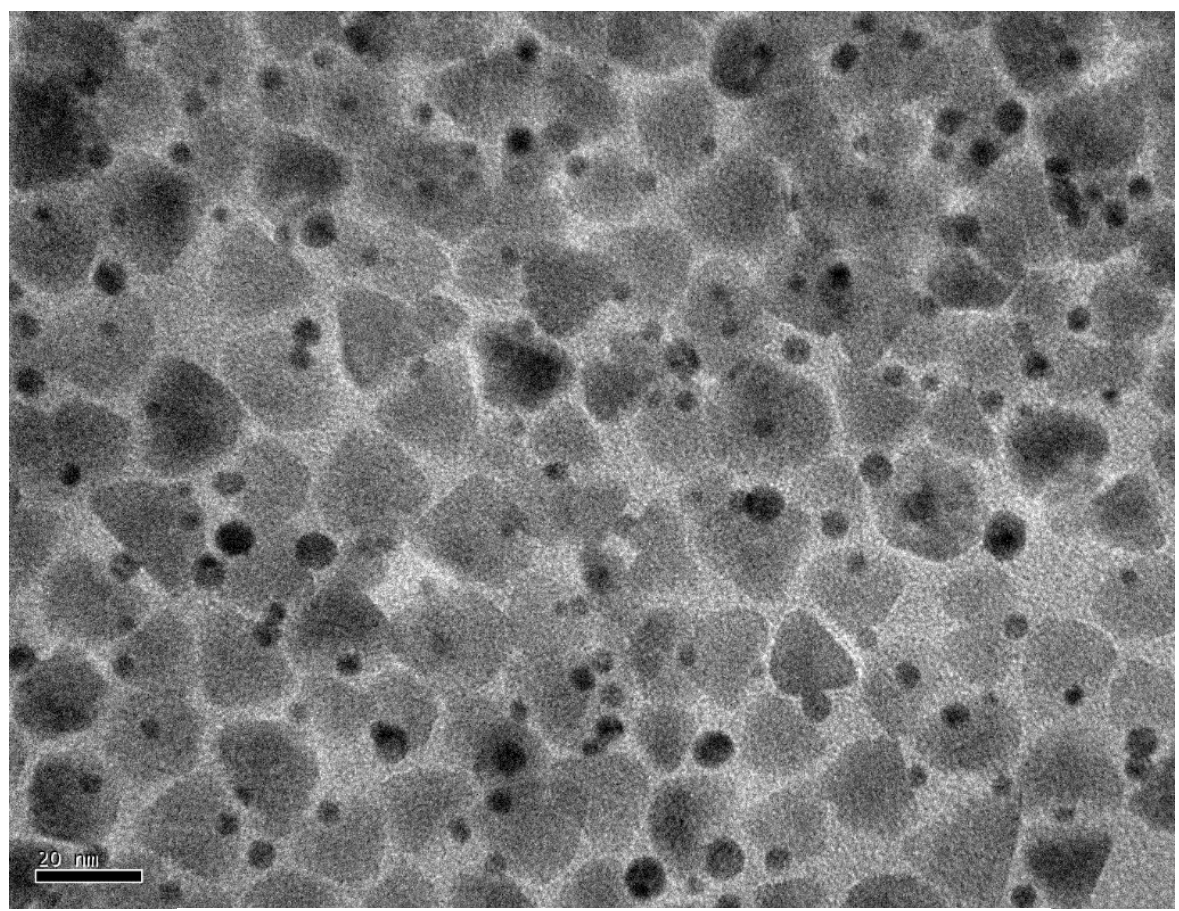

Figure 6.18 TEM images of Au-ZnO nanopyramids 
The HRTEM images in Figure 6.19 provide additional insight on the microstructure of the $\mathrm{Au}-\mathrm{ZnO}$ samples. The $\mathrm{ZnO}$ component of the heterostructure is single-crystalline with $\mathrm{ZnO}$ (002) lattice fringes observed. The Au can be identified as polycrystalline particles with multiple Au (111) facets observed having domain sizes of $\sim 2.6 \mathrm{~nm}$. This value agrees with the Au (111) domain size of $2.7 \mathrm{~nm}$ from the Rietveld analysis (see the section 6.4.3). The large number of $\mathrm{Au}$ (111) facets minimize the surface energy and strain due to the formation of twinned defects. ${ }^{11}$ The $\mathrm{ZnO}$ (002) plane appears to be parallel to the $\mathrm{Au}$ (111) plane at the interface. The basal surface of the $\mathrm{ZnO}$ structure is perpendicular to the $\mathrm{ZnO}$ (002) direction and the HRTEM images suggest the $\mathrm{ZnO}$ crystal starts growing in the [0001] direction, along the $c$-axis, on top of the Au seed. This growth mechanism was proposed by Flomin et al. ${ }^{1}$ using TEM images of aliquots obtained during different stages of the synthesis.

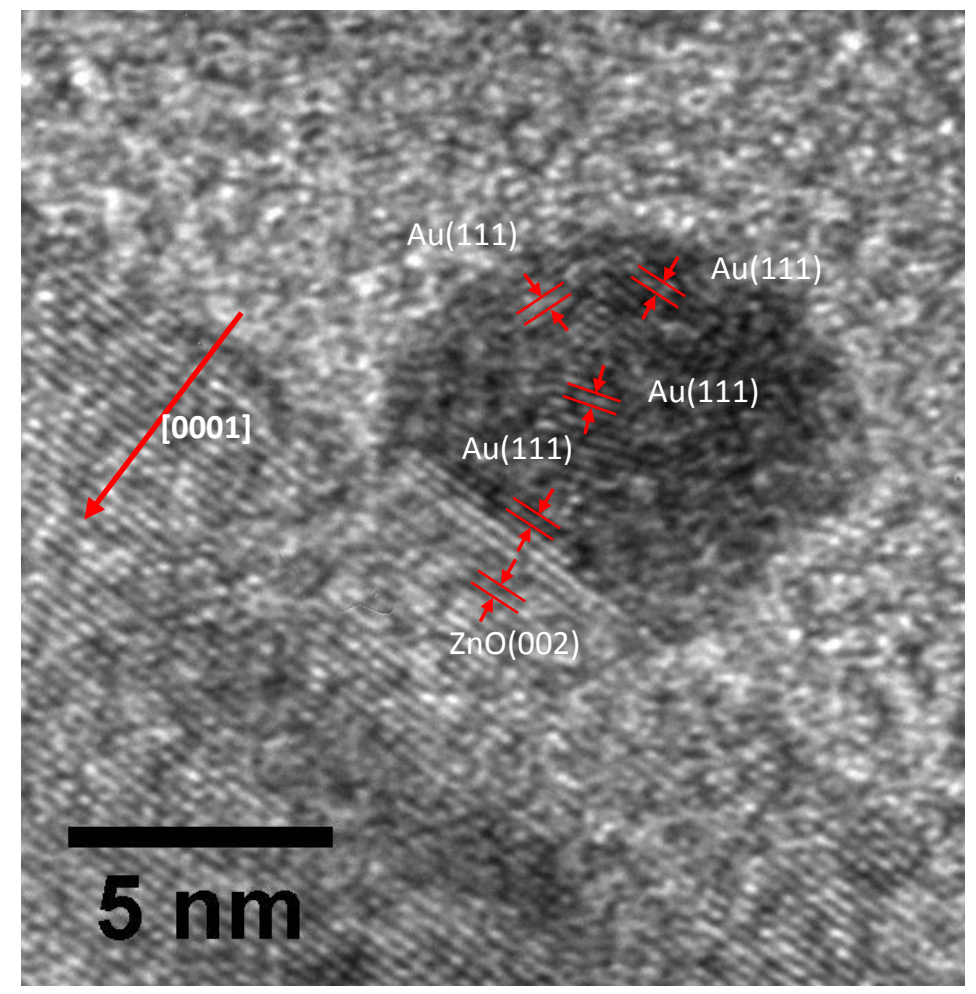

Figure 6.19 HRTEM image of the Au-ZnO interface 
TEM images of the $\mathrm{Au}-\mathrm{ZnO}$ heterostructures also suggest there is variation in the general position of the $\mathrm{Au}$ on the basal surface of the $\mathrm{ZnO}$ nanopyramid, which is shown in both the images and schematic of Figure 6.20. This variation has not been noted in previous structural characterizations of this interface ${ }^{1-3}$ and may result from the changes in surfactants used in the synthesis reported here. In general, it appears that the Au particle does not strictly occupy a position at the center of the $\mathrm{ZnO}$ basal plane and can instead occupy a variety of positions. Currently, the role of this structural detail on the photophysics of these samples is not understood.

The HRTEM images of $9 \mathrm{Au}-\mathrm{ZnO}$ interfaces were analyzed using ImageJ software. A rectangular area in the middle of the $\mathrm{ZnO}$ nanoparticle consisting of $2.5 \mathrm{~nm}$ width and 4 lattice planes of length was chosen for the analysis (Figure 6.21). The average $d$-spacing of these 4 lattice planes was used as an estimate of the $d$-spacing of the corresponding rectangular area. Up to 20 lattice planes from the $\mathrm{Au}-\mathrm{ZnO}$ interface were analyzed. The final results and standard deviations can be found in Table 6.2 and which are the average values of all $9 \mathrm{Au}-\mathrm{ZnO}$ interfaces. Figures 6.22, 6.23, 6.24 and 6.25 show the HRTEM images of additional Au-ZnO interfaces. 
(a)

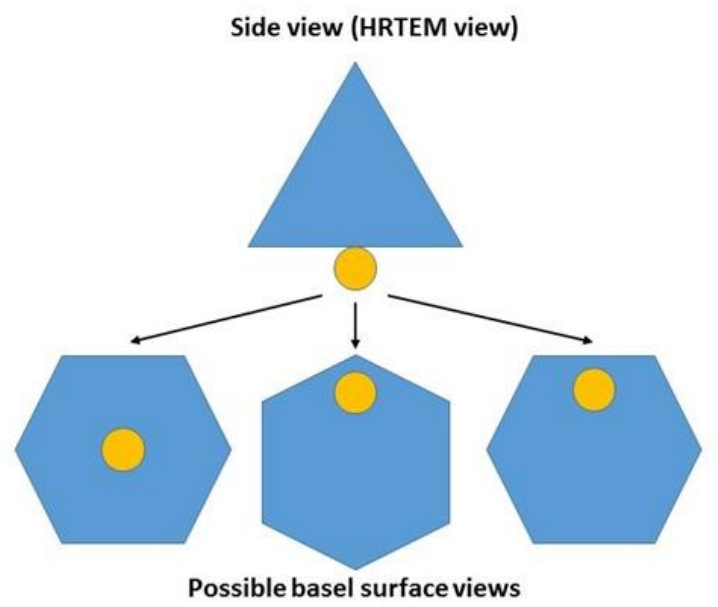

(b)

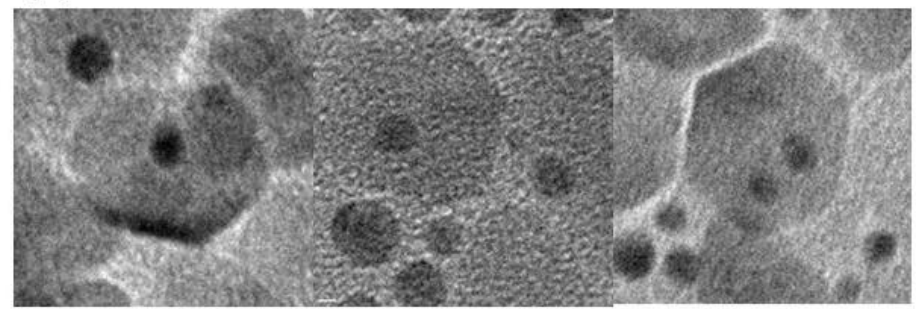

Figure 6.20 (a) Schematic diagram for possible orientations of Au nanoparticles on the basal surface of the $\mathrm{ZnO}$ nanopyramids (b) TEM images of different basal surfaces of the $\mathrm{ZnO}$ nanopyramids.

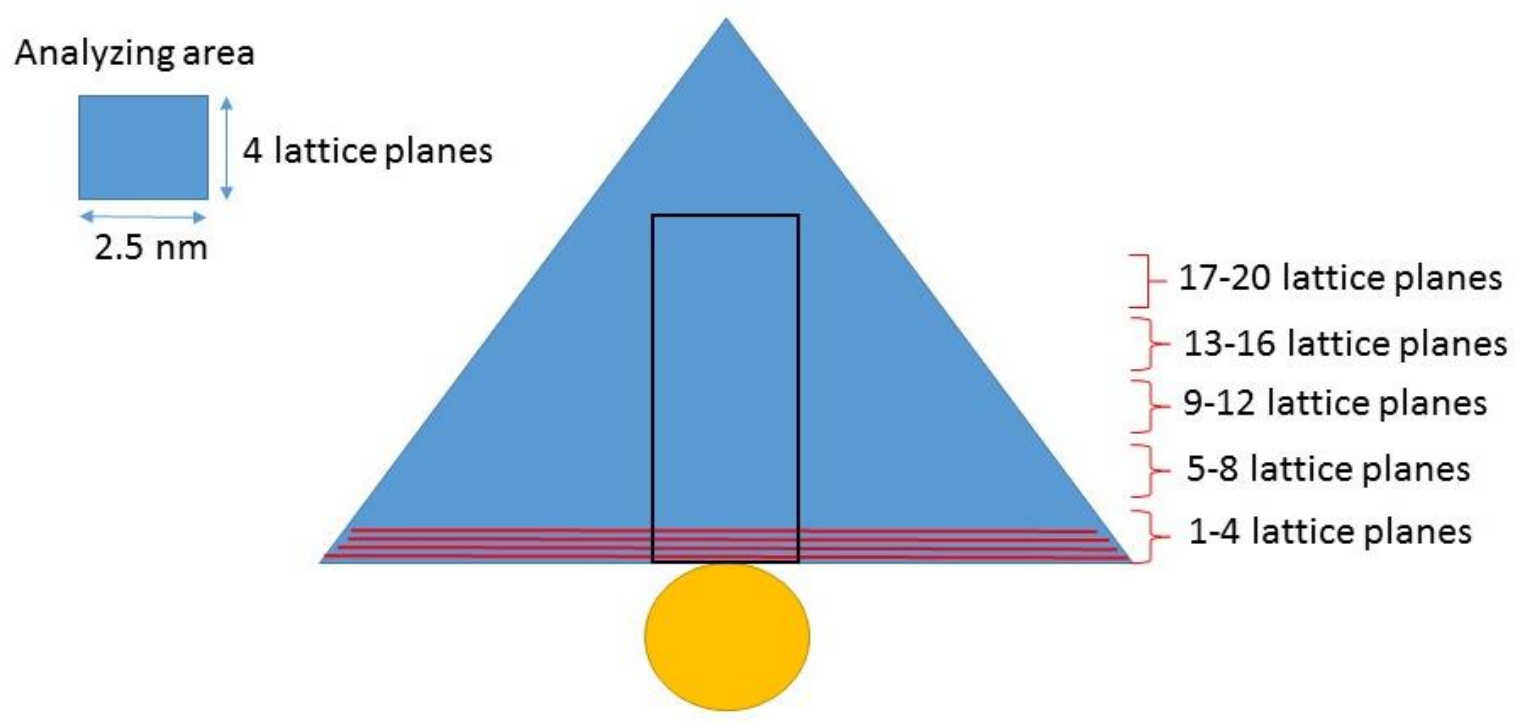

Figure 6.21 $d$-spacing analyzing method of $\mathrm{ZnO}(002)$ lattice plane 
Table 6.2 d-spacing values of $\mathrm{Au}-\mathrm{ZnO}$ interfaces

\begin{tabular}{|c|c|c|c|c|c|c|c|c|c|c|c|}
\hline \multirow{2}{*}{$\begin{array}{l}\text { Lattice } \\
\text { planes }\end{array}$} & \multicolumn{11}{|c|}{ d-spacing $(\AA)$} \\
\hline & I-1 & $\mathrm{I}-2$ & $\mathrm{I}-3$ & $\mathrm{I}-4$ & $\mathrm{I}-5$ & I-6 & $\mathrm{I}-7$ & $\mathrm{I}-8$ & I-9 & Average & $\begin{array}{l}\text { Standard } \\
\text { deviation }\end{array}$ \\
\hline $0-4$ & 2.65 & 2.75 & - & 2.72 & 2.70 & 2.65 & - & 2.65 & 2.70 & 2.69 & 0.04 \\
\hline $4-8$ & 2.62 & 2.55 & 2.67 & 2.60 & 2.55 & 2.70 & 2.60 & 2.77 & 2.70 & 2.64 & 0.07 \\
\hline $8-12$ & 2.72 & 2.67 & 2.55 & 2.55 & 2.57 & 2.55 & 2.70 & 2.70 & 2.62 & 2.63 & 0.07 \\
\hline $12-16$ & 2.57 & 2.60 & 2.60 & 2.57 & 2.60 & 2.62 & 2.72 & 2.72 & 2.62 & 2.62 & 0.06 \\
\hline $16-20$ & 2.62 & 2.55 & 2.60 & 2.67 & 2.57 & 2.55 & 2.60 & 2.50 & 2.65 & 2.59 & 0.05 \\
\hline
\end{tabular}

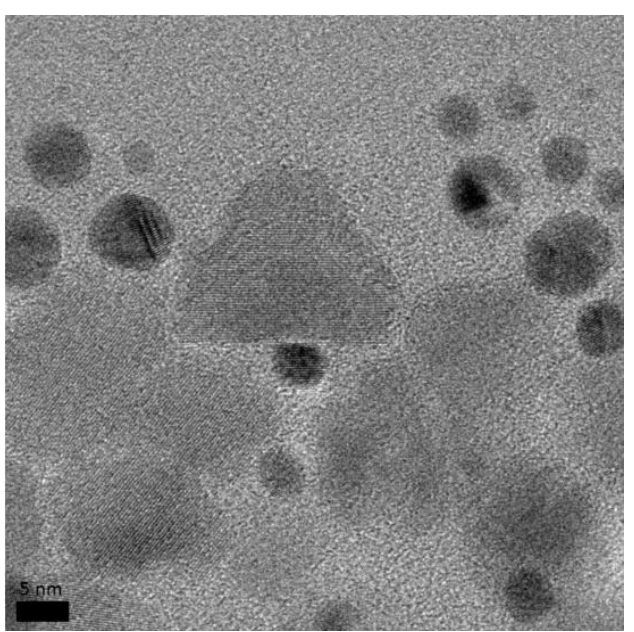

Figure 6.22 $\mathrm{Au}-\mathrm{ZnO}$ interface 1

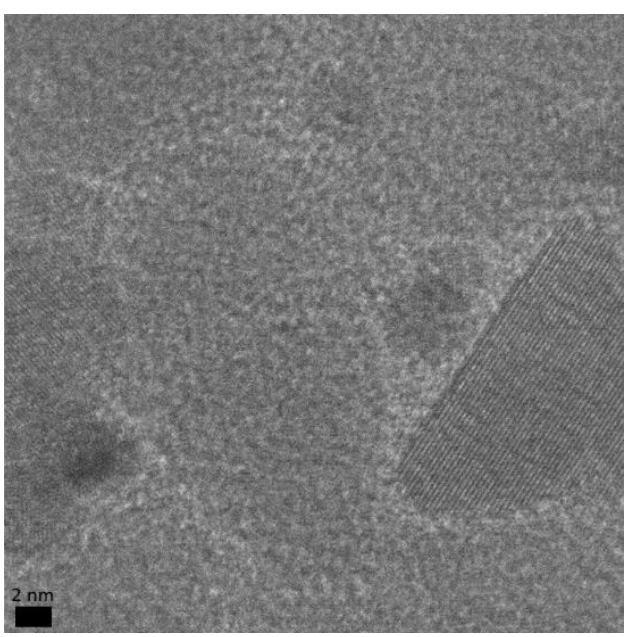

Figure 6.24 $\mathrm{Au}-\mathrm{ZnO}$ interface 3

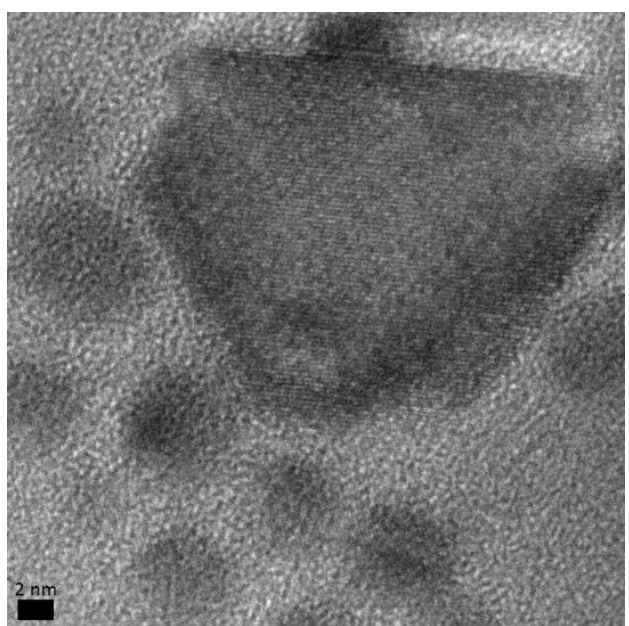

Figure 6.23 $\mathrm{Au}-\mathrm{ZnO}$ interface 2

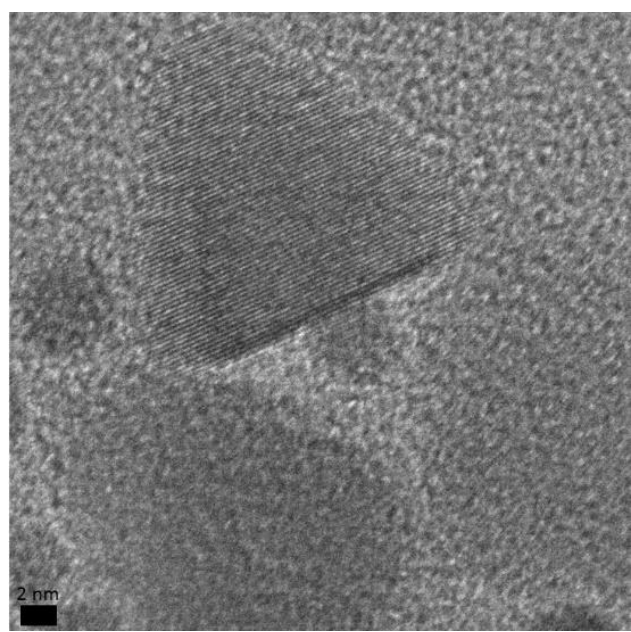

Figure 6.25 $\mathrm{Au}-\mathrm{ZnO}$ interface 4 
Interestingly, the $d$-spacing corresponding to the $\mathrm{ZnO}(002)$ plane experiences a $\sim 3.5 \%$ expansion relative to the bulk value of $2.60 \AA$ (Figure 6.26). This expansion has not been well documented in previous studies of $\mathrm{Au}-\mathrm{ZnO}$ made by similar synthetic approaches. ${ }^{1,2}$ This expansion only occurs within $\sim 1.5 \mathrm{~nm}$ of the $\mathrm{ZnO}$ interface with Au (Figure 6.27). This lattice expansion gradually decreases and completely disappears $\sim 4.5 \mathrm{~nm}$ away from the interface where the spacing approaches a constant bulk value of $2.60 \AA$. The plain $\mathrm{ZnO}$ nanoparticles do not experience any measurable lattice expansion relative to bulk values (not shown). For comparison, Shim et. al. ${ }^{12}$ have previously reported $\sim 1 \%$ lattice contraction in CdTe near the CdSe interface of linear barbell heterostructures. The lattice spacing of the CdTe tips on these barbells then approaches bulk values further away from the interface.

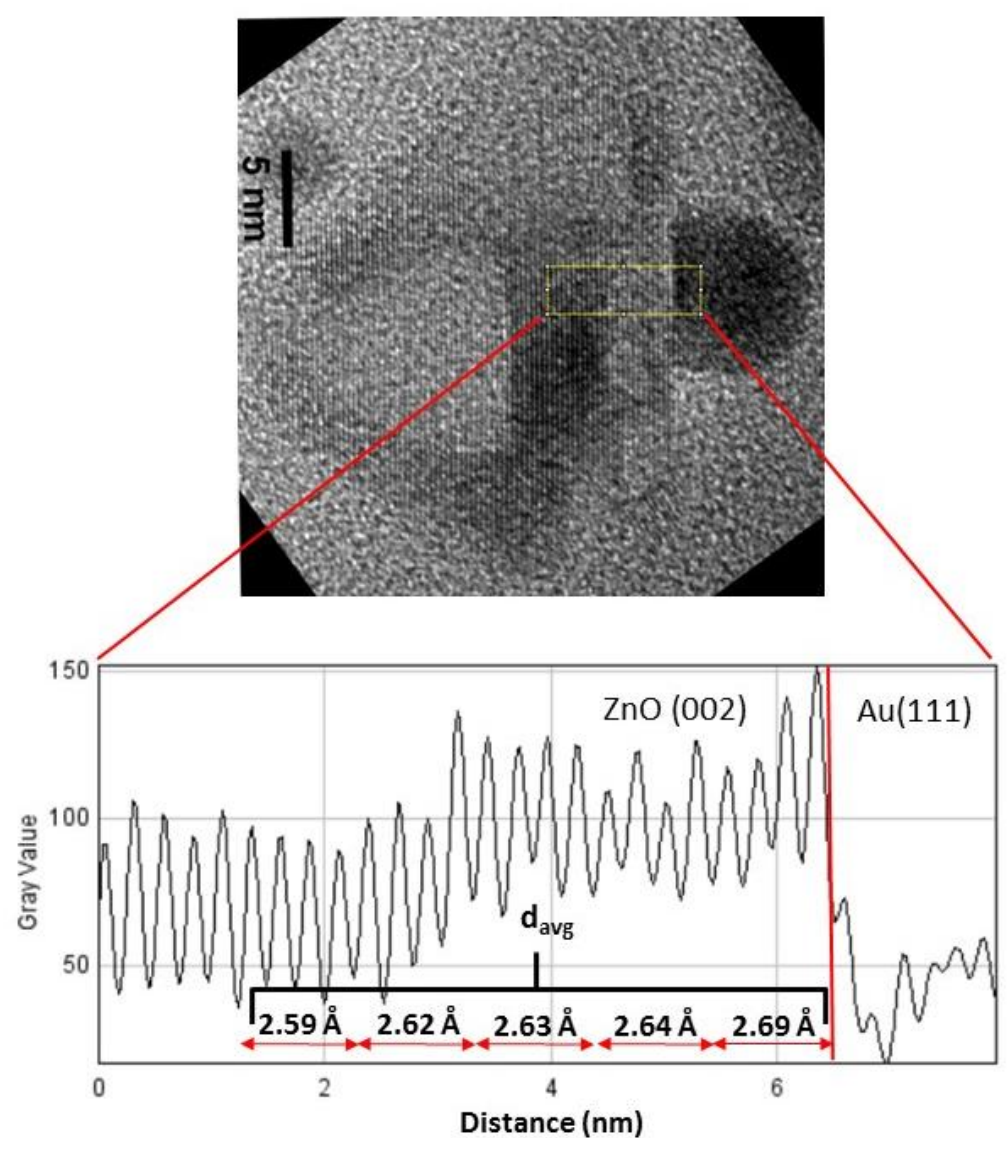

Figure 6.26 Line scan along the $\mathrm{Au}-\mathrm{ZnO}$ interface with average lattice spacings for the $\mathrm{ZnO}(002)$ plane in the regions shown. 


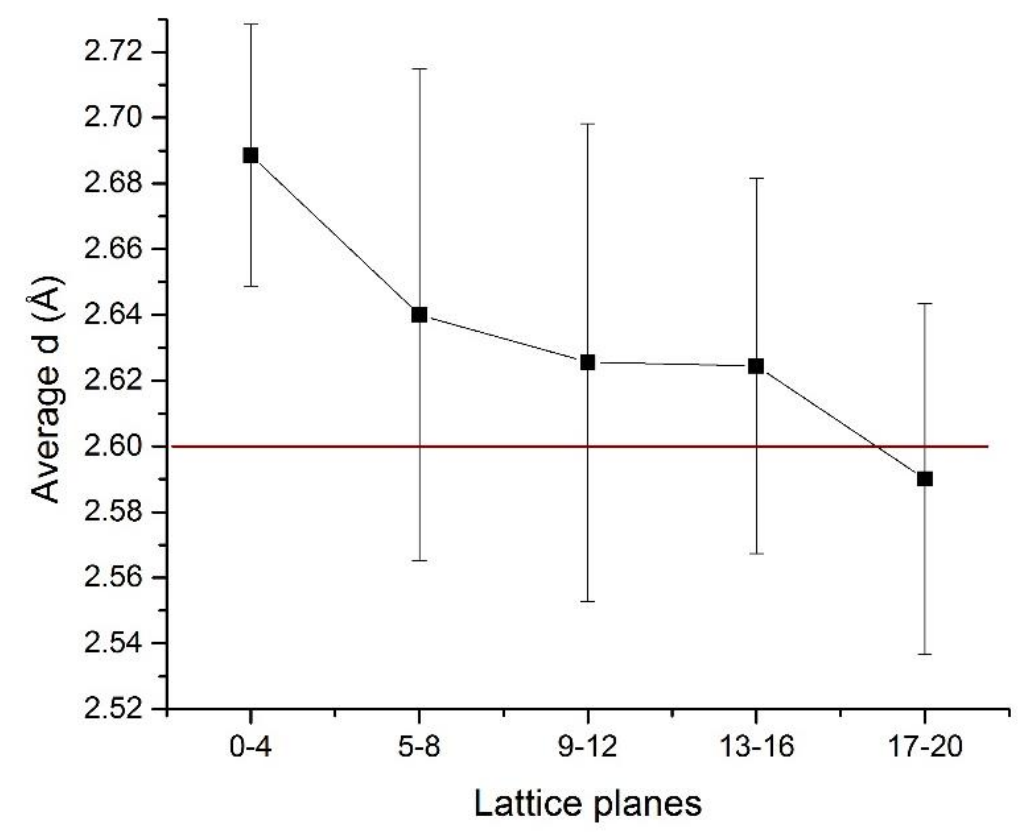

Figure 6.27 Average $d$-spacing of $\mathrm{ZnO}(002)$ at different distances from the interface. Error bars were determined by analyzing 9 different structures.

\subsubsection{Characterization of X-Ray Diffraction patterns and Rietveld analysis}

Powder XRD patterns confirm the cubic structure of Au nanoparticles and hexagonal wurtzite structure of $\mathrm{ZnO}$ (Figure 6.28). Rietveld analysis is performed to evaluate crystal size and microstrain in both pure $\mathrm{ZnO}$ and $\mathrm{Au}-\mathrm{ZnO}$ (Figures 6.29 and 6.30). While particle size determinations from a Rietveld analysis of XRD lineshapes are simple to interpret, the precise origin of microstrain contributions to linewidths is more challenging to attribute to a single structural feature. ${ }^{13-15}$ The microstrain value determined in a Rietveld analysis is interpreted as a generic deviation from the ideal lattice which can be caused by a variety of crystal-level defects, such as dislocations, domain boundaries, and the expansion/contraction of a lattice. As such, microstrain analysis can provide verification of and insight into some of the microstructure and defects noted in the TEM images above. 
The calculated Au particle size in the heterostructure is $2.7 \mathrm{~nm}$, which is consistent with the polycrystalline nature of the Au particles and the (111) domain sizes noted in Figure 6.19. The calculated particle size for the pure $\mathrm{ZnO}$ sample is $25.9 \mathrm{~nm}$, which is consistent with the TEM images of Figure 6.16. The calculated $\mathrm{ZnO}$ particle size in $\mathrm{Au}-\mathrm{ZnO}$ is 20.5 $\mathrm{nm}$ which also agrees well with the size determined from TEM images (Figure 6.18). The Rietveld analysis estimates a $0.324 \%$ microstrain for the $\mathrm{Au}$ in $\mathrm{Au}-\mathrm{ZnO}$ which provides evidence for the distribution of $\mathrm{Au}(111)$ domains noted in Figure 6.19. Microstrain estimates for pure $\mathrm{ZnO}$ were $0.047 \%$. The calculated microstrain for the $\mathrm{ZnO}$ in the $\mathrm{Au}$ $\mathrm{ZnO}$ sample is $0.146 \%$, which is 3 times larger than that for pure $\mathrm{ZnO}$. In comparison, Khusaimi et al. ${ }^{16}$ previously reported a $0.257 \%$ microstrain of $\mathrm{ZnO}$ in $\mathrm{Au} / \mathrm{ZnO}$ heterostructures on a Si substrate.

Microstrain for $\mathrm{ZnO}$ in the heterostructures can be attributed the increased to the lattice expansion noted in Figures 6.26 and 6.27. The XRD measurement is a bulk probe and averages this defect over the entire sample, including regions where $\mathrm{ZnO}$ retains its normal lattice, which likely reduces the magnitude of the microstrain estimate. In fact, the average lattice expansion in Figure 6.26 using the entire $\mathrm{ZnO}$ portion of the image (interface and non-interface regions) is only $\sim 0.38 \%$, compared to $\sim 3.5 \%$ at the interface, which illustrates how easily the structural characteristics of the interface can be averaged out. 


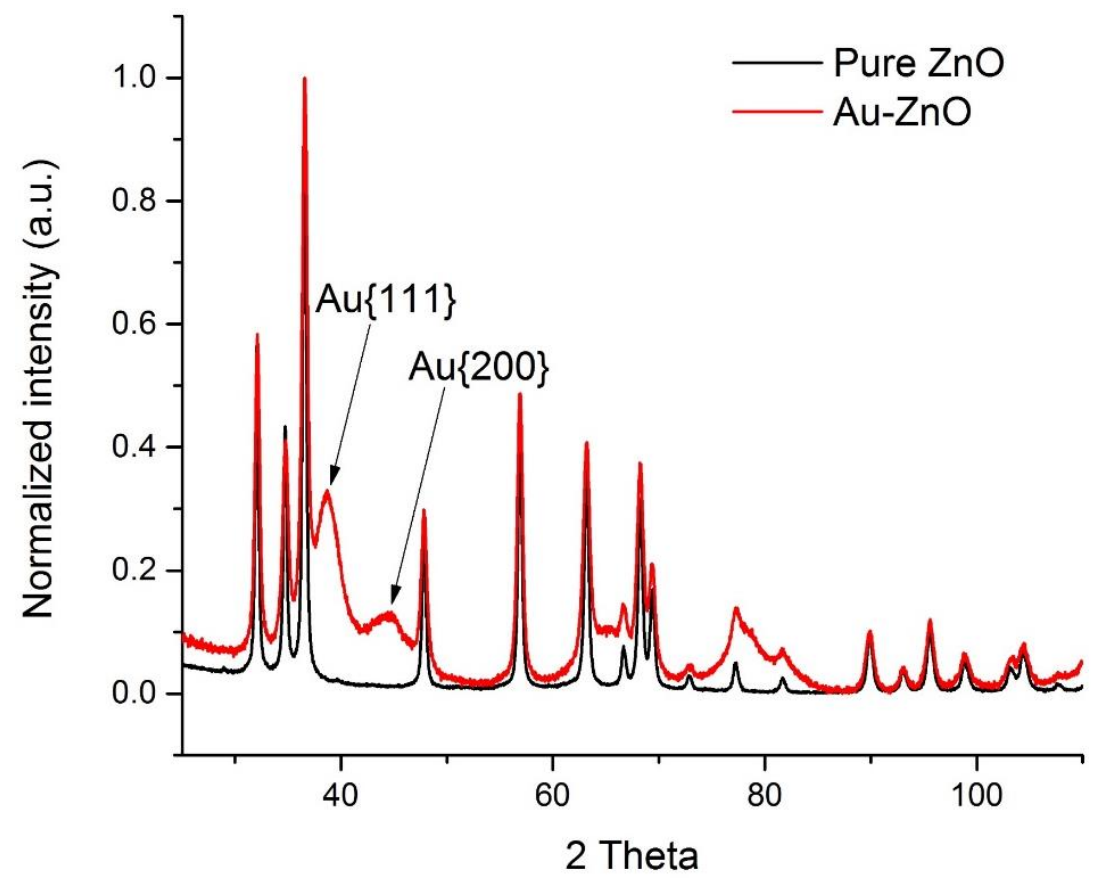

Figure 6.28 Comparison of $\mathrm{X}$-ray diffraction patterns of pure $\mathrm{ZnO}$ and $\mathrm{Au}-\mathrm{ZnO}$ nanopyramids

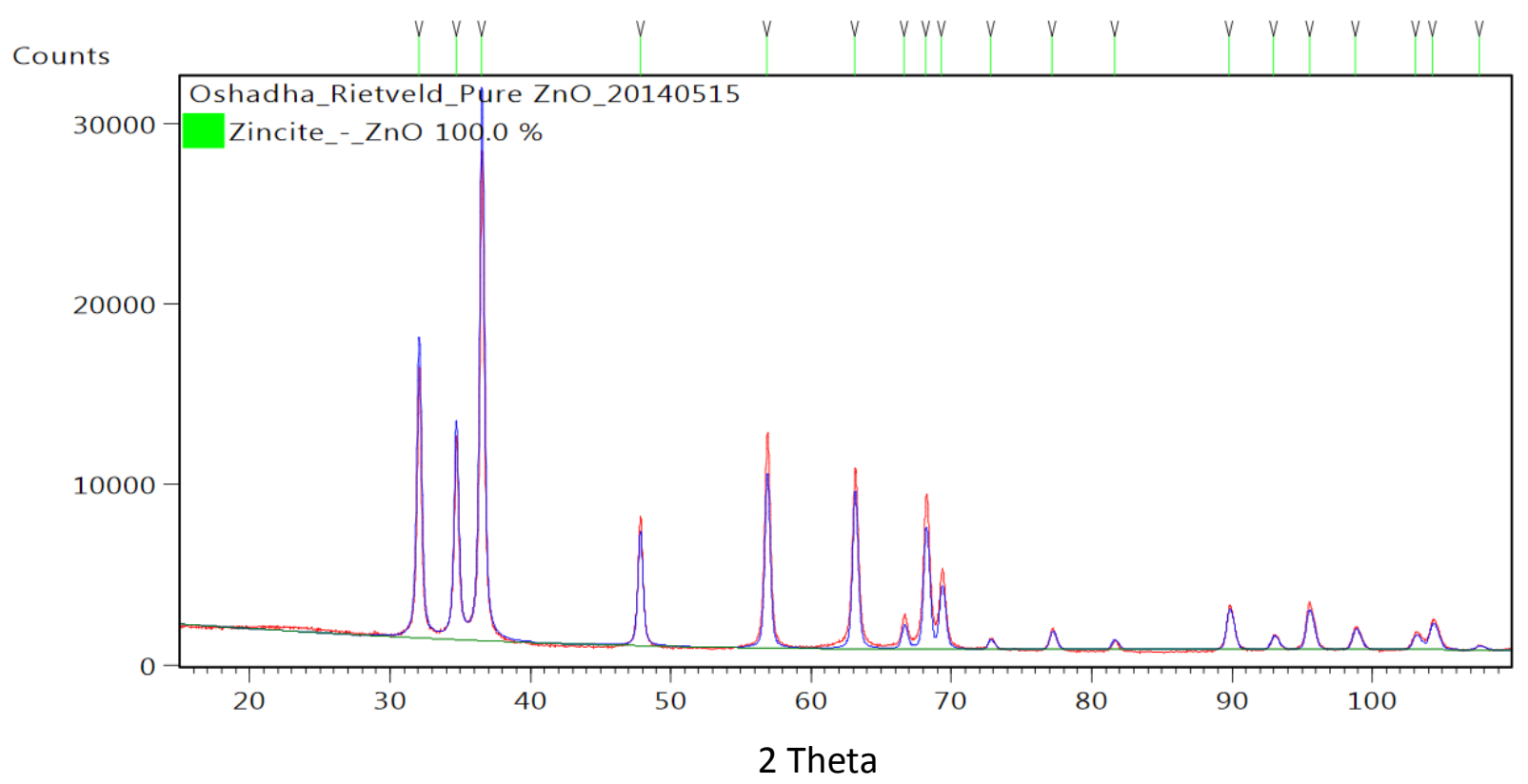

Figure 6.29 High resolution XRD patterns with calculated Rietveld pattern for pure $\mathrm{ZnO}$ 


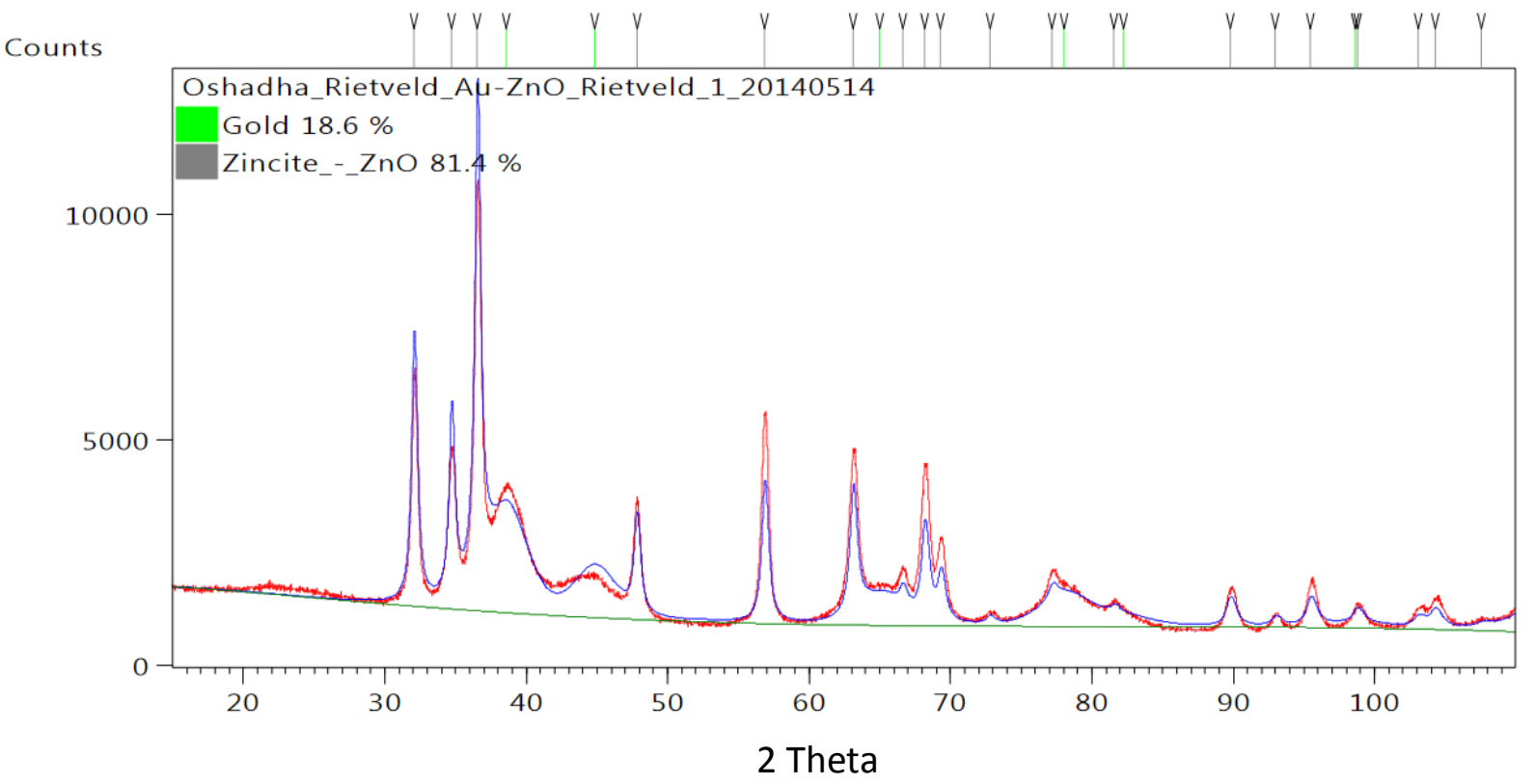

Figure 6.30 High resolution XRD patterns with calculated Rietveld pattern for Au-ZnO

\subsubsection{Photocatalytic performance}

Figure 6.31 shows representative UV-Vis absorption spectra used to determine the kinetics of MB photodegradation under $\lambda>295 \mathrm{~nm}$ irradiation. Figure 6.32 shows photodegradation kinetics for pure $\mathrm{ZnO}, \mathrm{Au}-\mathrm{ZnO}, \mathrm{Au}-\mathrm{SiO}_{2}$ and a stock solution of $20 \mu \mathrm{mol} / \mathrm{L} \mathrm{MB}$ (no catalyst) also at $\lambda>295 \mathrm{~nm}$. After 60 minutes of illumination, Au- $\mathrm{ZnO}$ photodegrades $\sim 98$ $\%$ of the MB. In comparison, pure $\mathrm{ZnO}$ only degrades $\sim 17 \%$, the $\mathrm{Au} / \mathrm{SiO}_{2}$ control $\sim 4 \%$, and the stock solution of $\mathrm{MB}$ (without catalyst) $\sim 10 \%$. Plots of $\ln \left(C / C_{0}\right)$ versus time are linear (Figure 6.33) which confirm the pseudo first order reaction kinetics and associated degradation mechanisms noted in many MB photodecomposition studies. ${ }^{8}$ The exceptional activity for $\mathrm{Au}-\mathrm{ZnO}$ combined with the low or lack of activity for $\mathrm{ZnO}, \mathrm{Au}-\mathrm{SiO}_{2}$ and $\mathrm{MB}$ alone, point to the synergistic role of the $\mathrm{Au}$ and $\mathrm{ZnO}$ in the heterostructured materials. 


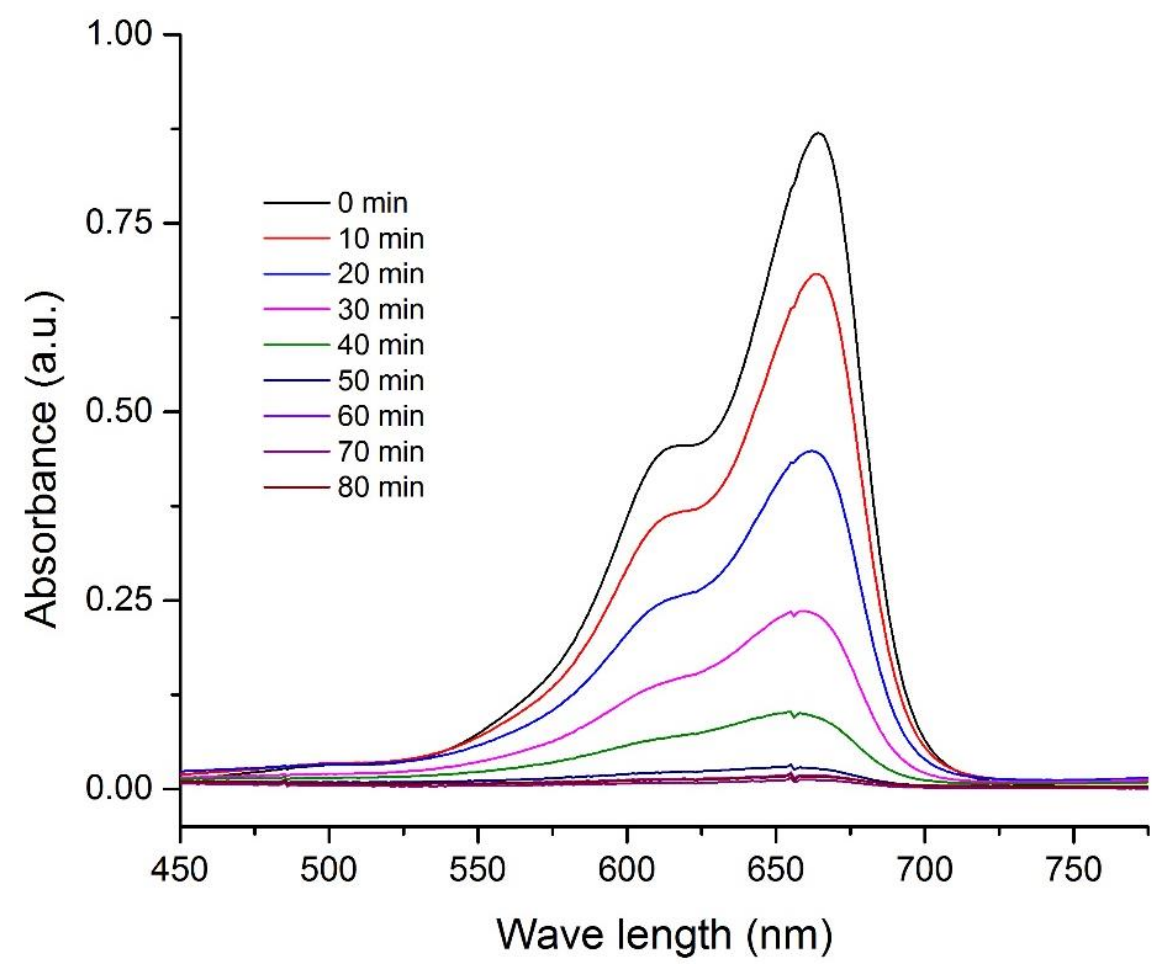

Figure 6.31 UV-Vis absorption spectra for MB degradation using Au-ZnO with $\lambda>295 \mathrm{~nm}$

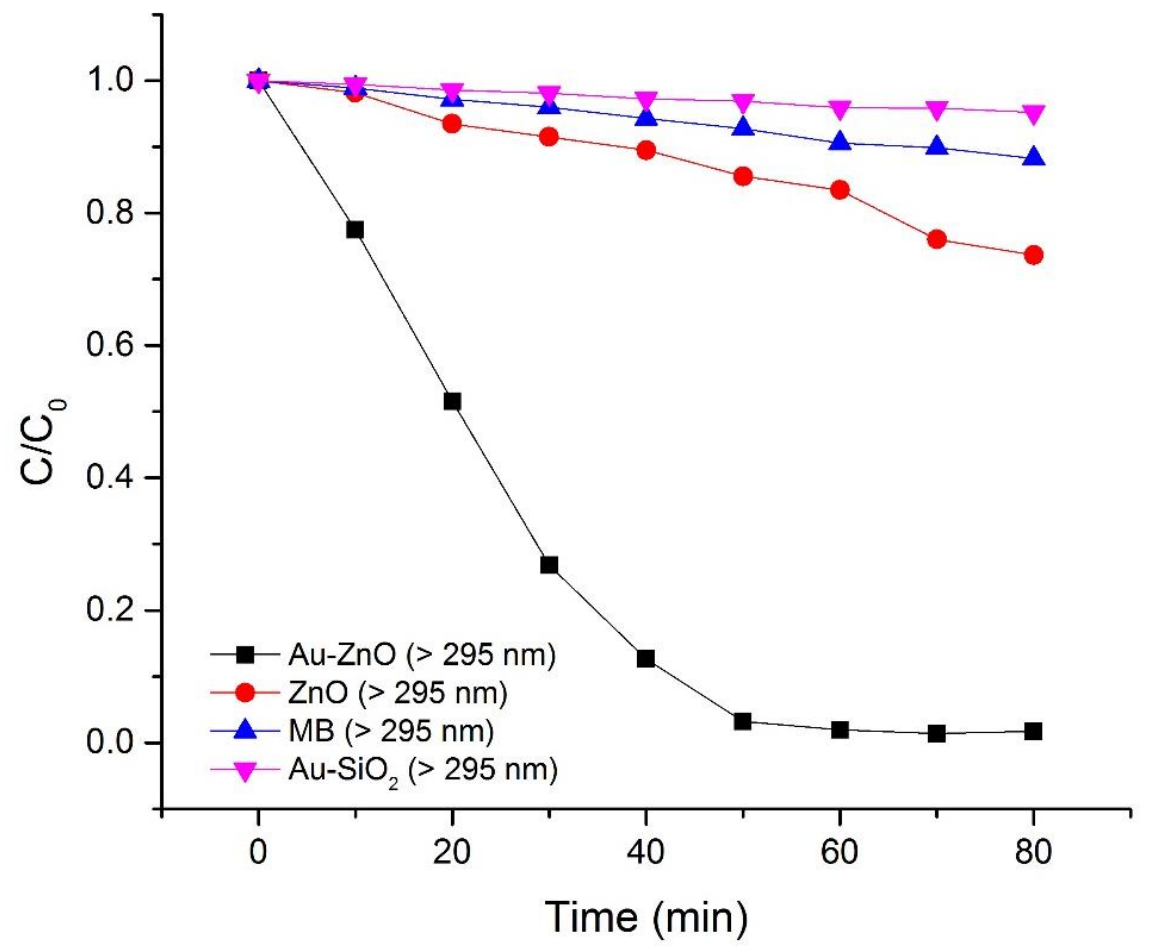

Figure 6.32 $\mathrm{MB}$ degradation results for $\mathrm{Au}-\mathrm{ZnO}$ (black), $\mathrm{ZnO}$ (red), $\mathrm{MB}$ solution (no catalyst) (blue) and $\mathrm{Au}-\mathrm{SiO}_{2}$ (magenta) with $\lambda>295 \mathrm{~nm}$ illumination 
(a)

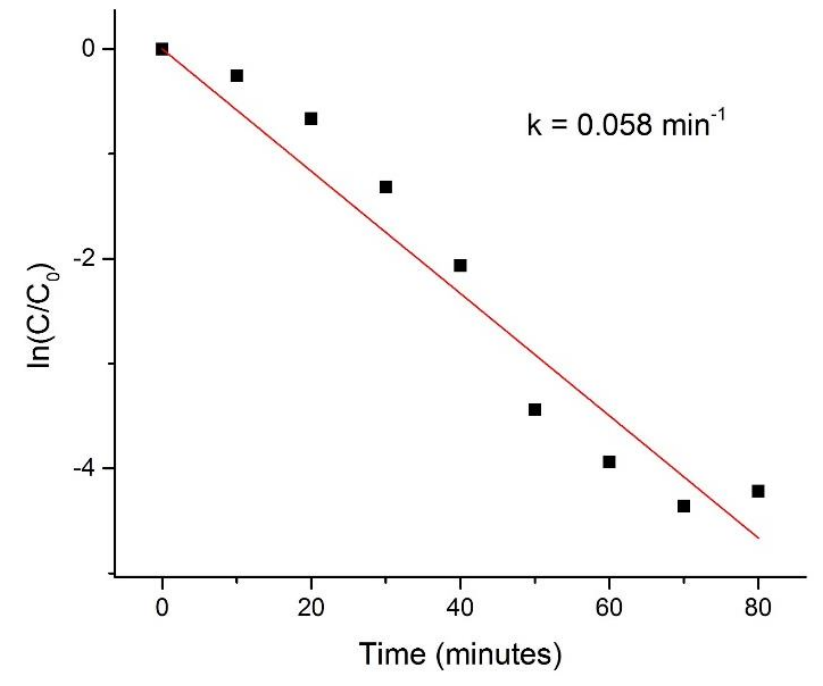

(b)

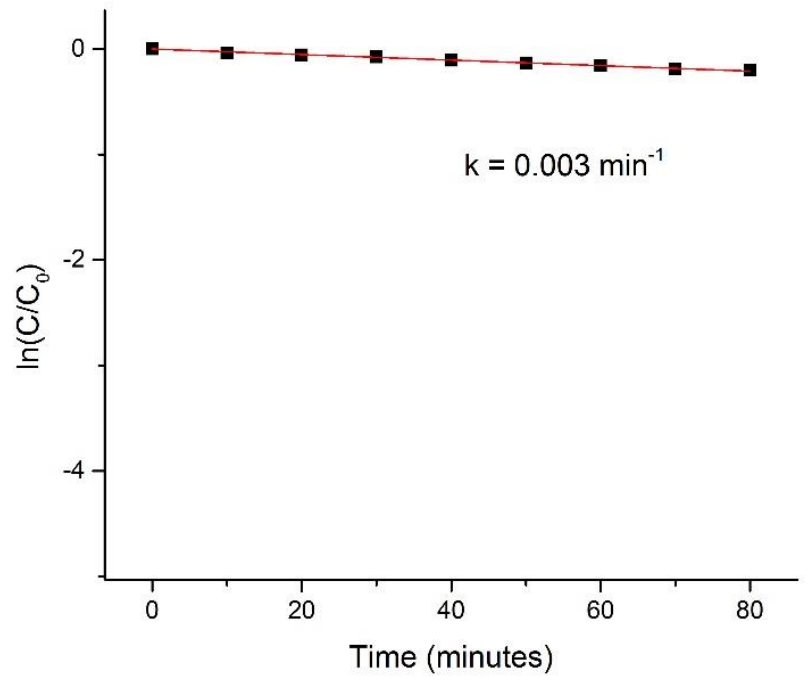

Figure 6.33 Linear fit for $\ln \left(C / C_{0}\right)$ of $\mathrm{MB}$ versus time for $\mathrm{Au}-\mathrm{ZnO}$ (a) under $\lambda>295 \mathrm{~nm}$ illumination (b) under $\lambda>400 \mathrm{~nm}$ illumination

Wavelength dependent photodecomposition studies were employed to help answer mechanistic questions about the photophysics of these samples. Figure 6.34 shows photodegradation kinetics of MB using $\lambda>295 \mathrm{~nm}, \lambda>320 \mathrm{~nm}$ and $\lambda>400 \mathrm{~nm}$ longpass filters. Figure 6.35, illustrates that the catalytic activity of $\mathrm{Au}-\mathrm{ZnO}$ remains almost constant between $295 \mathrm{~nm}<\lambda<320 \mathrm{~nm}$. But for $\lambda>400 \mathrm{~nm}$, Au-ZnO has no appreciable activity. The calculated pseudo first order rate constants of Au-ZnO were $0.058 \mathrm{~min}^{-1}, 0.059 \mathrm{~min}^{-1}$ and $0.003 \mathrm{~min}^{-1}$ for $\lambda>295 \mathrm{~nm}, \lambda>320 \mathrm{~nm}$ and $\lambda>400 \mathrm{~nm}$ illumination, respectively. This confirms the necessity of direct bandgap excitation of $\mathrm{ZnO}$ in the $\mathrm{Au}-\mathrm{ZnO}$ heterostructure (bandgap $383 \mathrm{~nm}$, Figure 6.15) in order to initiate the photodecomposition of MB. P25 $\mathrm{TiO}_{2}$ has comparable kinetics for MB degradation (Figure 6.35) for $\lambda>295$ $\mathrm{nm}$, but this activity approaches zero for $\lambda>320 \mathrm{~nm}$. P25 $\mathrm{TiO}_{2}$ has a comparable bandgap to $\mathrm{ZnO}$ and we suspect that the large difference in activity towards $\mathrm{MB}$ results, in part, from 
the lack of metal co-catalysts on the $\mathrm{TiO}_{2}$ sample for shuttling electrons into solution. In addition, the $\mathrm{Au}-\mathrm{ZnO}$ heterostructures have subtle changes in electronic structure and band alignments (discussed below) that also likely contribute to their improved activity over P25 $\mathrm{TiO}_{2}$.

Several previous works have reported higher catalytic activity of $\mathrm{Au}-\mathrm{ZnO}$ heterostructures over pure $\mathrm{ZnO}$ using dye degradation reactions. ${ }^{1-4,8,17-24}$ Direct comparisons of these efforts and our own are difficult due to different reaction conditions and dyes used. One reasonable method for comparing these works involves evaluating the differences in $\%$ decomposition between the $\mathrm{ZnO}$ and $\mathrm{Au}-\mathrm{ZnO}$ catalysts. Yao et al. ${ }^{3}$ showed that methyl orange decomposition was $\sim 2$ times faster with $\mathrm{Au}-\mathrm{ZnO}$ over $\mathrm{ZnO}$. Li et al. ${ }^{2}$ and Wang et al. ${ }^{22}$ showed that rhodamine $\mathrm{B}$ decomposition was $\sim 1.5$ and $\sim 3$ times faster with $\mathrm{Au}-\mathrm{ZnO}$ versus $\mathrm{ZnO}$ respectively. Lee et al. ${ }^{19}$ showed that the degradation of rhodamine $6 \mathrm{G}$ was $\sim 2$ times faster with $\mathrm{Au}-\mathrm{ZnO}$ over $\mathrm{ZnO}$. In our current work, the degradation of methylene blue was 6 times faster with $\mathrm{Au}-\mathrm{ZnO}$ than pure $\mathrm{ZnO}$. 


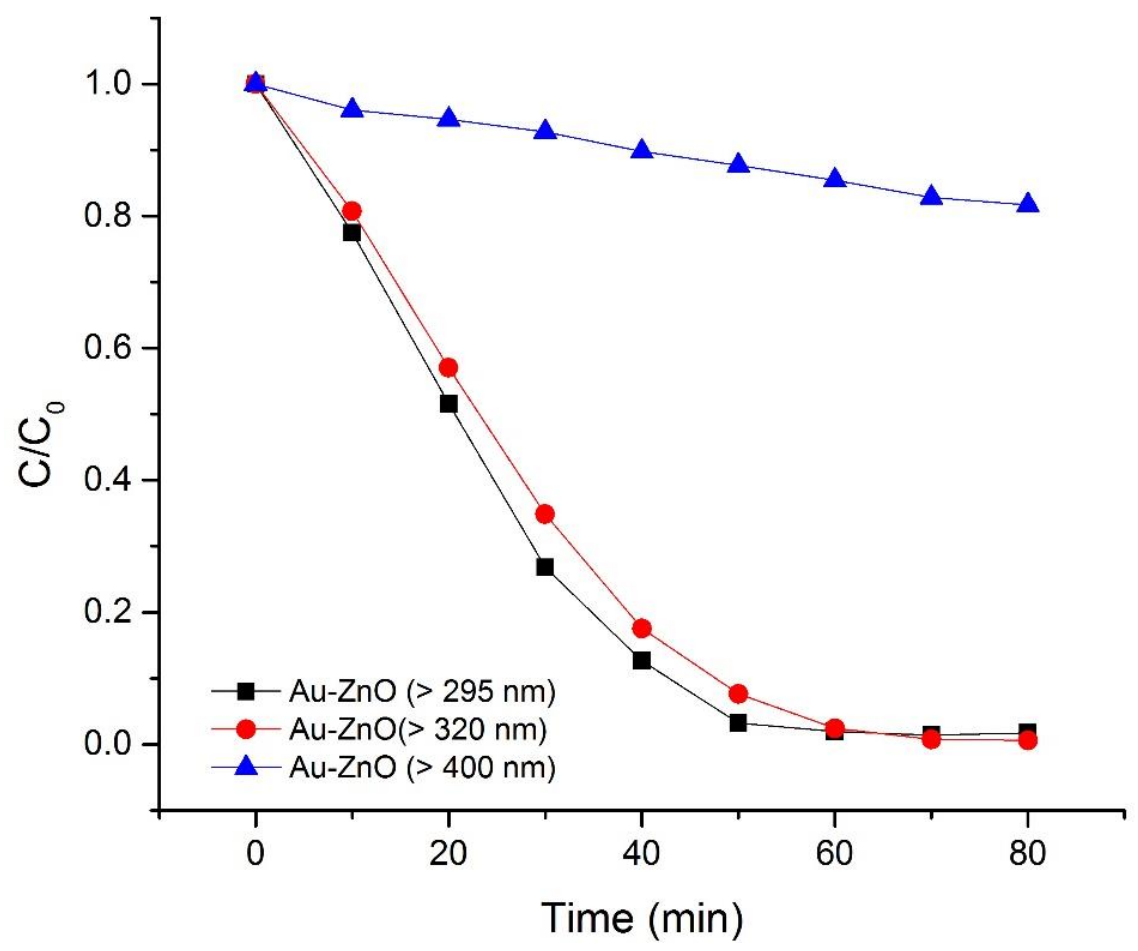

Figure 6.34 Wavelength dependent MB degradation with $\mathrm{Au}-\mathrm{ZnO}$

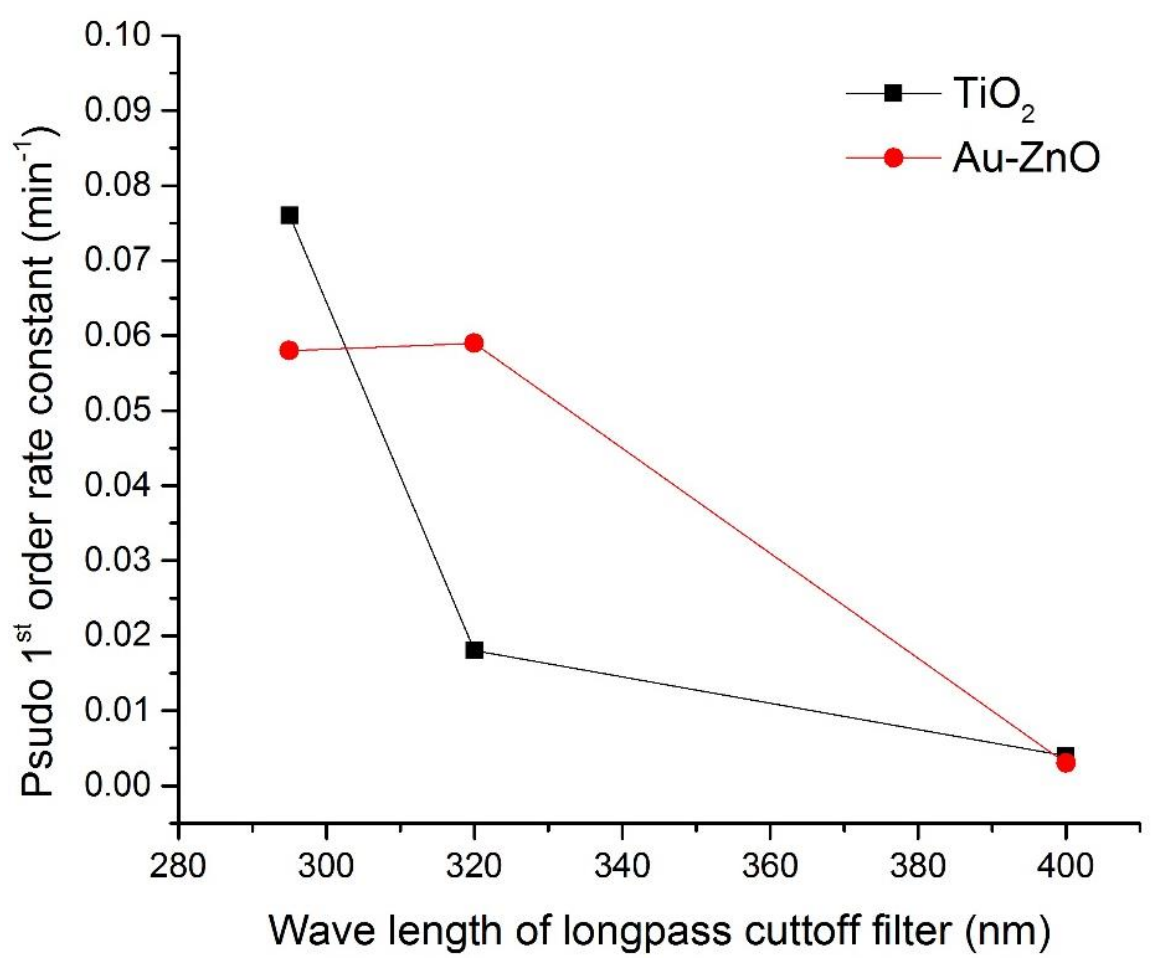

Figure 6.35 First order rate constants for $\mathrm{MB}$ degradation with $\mathrm{Au}-\mathrm{ZnO}$ and $\mathrm{TiO}_{2}$ using different longpass filters 
It is interesting to analyse reasons for the enhanced photocatalytic activity of $\mathrm{Au}-\mathrm{ZnO}$ over $\mathrm{ZnO}$. Plasmonic heating, ${ }^{25}$ direct electron injection ${ }^{26}$ from $\mathrm{Au}$, and field-enhancement created by $\mathrm{Au}$ plasmon resonance ${ }^{27}$, can all be easily ruled out from the wavelength dependent studies of Figure 6.34. If the Au plasmon was playing a critical role in the photophysics of this sample, one would expect to see photocatalytic activity at wavelengths longer than $400 \mathrm{~nm}$. The loss of activity towards $\mathrm{MB}$ as the irradiation wavelength becomes sub-bandgap clearly points to a mechanism where direct gap excitation generates charge carriers that eventually create the radical species in solution that degrade MB. The lack of catalytic activity seen for $\mathrm{Au}$ nanoparticles on $\mathrm{SiO}_{2}$ (Figure 6.32) helps to rule out any potential mechanisms involving interband transitions from occupied $d$-bands below the Fermi level $\left(E_{\mathrm{f}}\right)$ to unoccupied $s-p$ bands above the $E_{\mathrm{f}}$ of $\mathrm{Au}$, as was observed experimentally by $\mathrm{Hou}$ et al. ${ }^{27}$ for the photocatalytic reduction of $\mathrm{CO}_{2}$ with $\mathrm{Au} / \mathrm{TiO}_{2}$.

The characterization and photocatalytic activity data presented above allow us to propose how Au enhances the photoactivity of the heterostructures towards MB degradation (Figure 6.36). Direct gap excitation creates electrons and holes, which thermalize to the bottom and top of the conduction and valence bands, respectively, of $\mathrm{ZnO}$. The energy of the conduction band minimum and that of the valence band maximum of $\mathrm{ZnO}$ are $-0.2 \mathrm{~V}$ and +3.0 V respectively, with respect to the Normal Hydrogen Electrode (NHE). ${ }^{28}$ The Fermi level of $\mathrm{Au}$ is $+0.45 \mathrm{~V}$ with respect to NHE. ${ }^{4}$ Since the Fermi level of $\mathrm{Au}$ is below the conduction band of $\mathrm{ZnO}$, the photoexcited electrons are easily transferred to the $\mathrm{Au}$ nanoparticles from the conduction band of $\mathrm{ZnO} .^{22}$ The close contact of the $\mathrm{ZnO}$ and $\mathrm{Au}$ nanoparticles increases this charge separation efficiency helping to reduce carrier 
recombination. ${ }^{4}$ The build-up of electrons in Au will shift $E_{\mathrm{f}}$ in a more negative direction but the electron transfer from $\mathrm{ZnO}$ to $\mathrm{Au}$ would not be altered until $E_{\mathrm{f}}$ approaches the conduction band minimum of $\mathrm{ZnO}$. The electrons at the $E_{\mathrm{f}}$ of $\mathrm{Au}$ can be injected into $\mathrm{H}_{2} \mathrm{O}$ species to form $\mathrm{OH}^{*}$ radicals. The charge separation described above also facilitates injection of the hole left behind in the valence band into water to generate $\mathrm{OH}^{\bullet}$ radicals, which attack MB directly and contribute to the photodegradation. The products observed from this photodegradation mechanism of MB have been identified in previous works as $\mathrm{CO}_{2}$ gas, nitrate, sulfate and ammonium. ${ }^{29}$

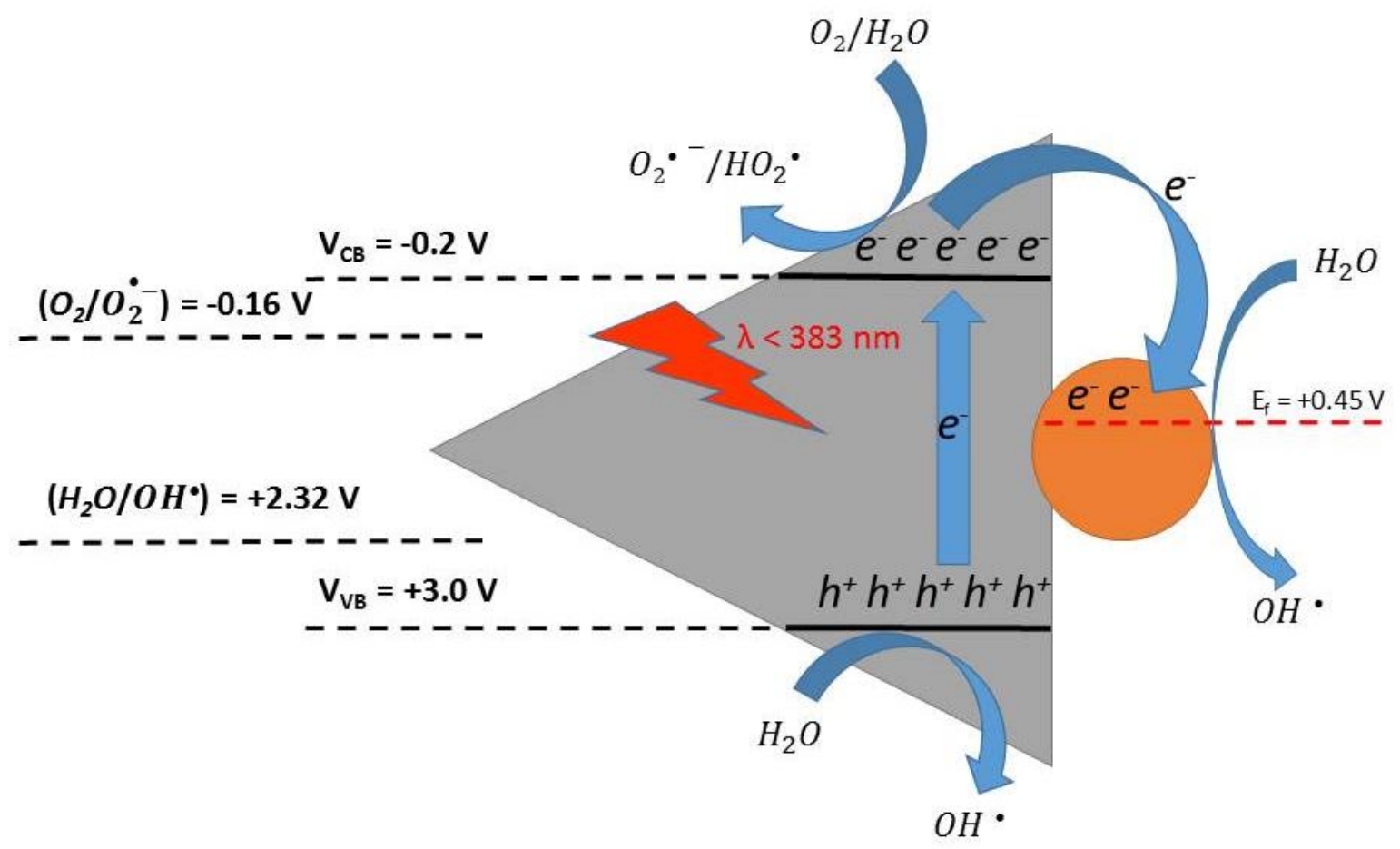

Figure 6.36 Approximate band alignment of $\mathrm{Au}-\mathrm{ZnO}$. Potentials are with respect to NHE 
The photocatalytic degradation of methylene blue in water can be explained according to the mechanism proposed by Houas et $a l^{29}$ as follows:

For $\lambda<359 \mathrm{~nm}$ (band gap of $\mathrm{ZnO}$ ) illumination,

$$
Z n O+h v \rightarrow Z n O\left[e_{C B}^{-}+h_{V B}^{+}\right]
$$

At valence band of $\mathrm{ZnO}$,

$$
\begin{gathered}
\mathrm{H}_{2} \mathrm{O} \Leftrightarrow \mathrm{H}^{+}+\mathrm{OH}^{-}- \\
\mathrm{ZnO}\left[h_{V B}^{+}\right]+\mathrm{OH}^{-} \rightarrow \boldsymbol{O H}^{\circ}
\end{gathered}
$$

At conduction band of $\mathrm{ZnO}$,

$$
\begin{gathered}
\mathrm{ZnO}\left[e_{\mathrm{CB}}^{-}\right]+\mathrm{O}_{2} \rightarrow \mathrm{O}_{2}^{\circ-}- \\
\mathrm{H}_{2} \mathrm{O} \Leftrightarrow \mathrm{H}^{+}+\mathrm{OH}^{-}- \\
\mathrm{O}_{2}^{\circ-}+\mathrm{H}^{+} \rightarrow \mathrm{HO}_{2}^{\circ}- \\
2 \mathrm{HO}_{2}^{\circ} \rightarrow \mathrm{H}_{2} \mathrm{O}_{2}+\mathrm{O}_{2} \\
\mathrm{ZnO}\left[e_{\mathrm{CB}}^{-}\right]+\mathrm{H}_{2} \mathrm{O}_{2} \rightarrow \boldsymbol{O H}^{\circ}+\mathrm{OH}^{-}
\end{gathered}
$$

At Au NP surface,

$$
\begin{gathered}
{\left[\text { hot } e^{-}\right]_{A u N P}+O_{2} \rightarrow O_{2}^{\circ-}} \\
H_{2} O \Leftrightarrow H^{+}+\mathrm{OH}^{-}---
\end{gathered}
$$




$$
\begin{aligned}
& \mathrm{O}_{2}^{\circ-}+\mathrm{H}^{+} \longrightarrow \mathrm{HO}_{2}^{\circ} \\
& 2 \mathrm{HO}_{2}^{\circ} \rightarrow \mathrm{H}_{2} \mathrm{O}_{2}+\mathrm{O}_{2} \\
& {\left[\text { hot } e^{-}\right]_{A u N P}+\mathrm{H}_{2} \mathrm{O}_{2} \rightarrow \mathrm{OH}^{\circ}+\mathrm{OH}^{-}}
\end{aligned}
$$

At MB molecules,

$$
M B+\boldsymbol{O H}^{\circ} \rightarrow M B \text { degradation products }
$$

In addition to charge separation, the $\mathrm{Au}$ in heterostructures is known to decrease the work function of a metal oxide, which can impact carrier generation and injection. ${ }^{30}$ Sykes et al. ${ }^{30}$ have demonstrated that the work function of $\mathrm{TiO}_{2}$ can decrease from $5.3 \mathrm{eV}$ to $4.8 \mathrm{eV}$ with increasing Au loading due to an interfacial dipole created as charge flows from $\mathrm{Au}$ to $\mathrm{TiO}_{2}$ in order to equilibrate Fermi levels. The work function of $\mathrm{ZnO}$ and that of $\mathrm{Au}$ are $5.3 \mathrm{eV}$ and $5.1 \mathrm{eV}$, respectively, which indicates a similar mechanism could occur with these heterostructures. ${ }^{31,32}$ The lowered $\mathrm{ZnO}$ work function caused by equilibration with the $\mathrm{Au}$ Fermi level would increase the transfer rate of conduction band electrons from the $\mathrm{ZnO}$ surface to adsorbed $\mathrm{O}_{2}$ (rate limiting step of the photocatalytic $\mathrm{MB}$ degradation) and $\mathrm{H}_{2} \mathrm{O}$ to form $\mathrm{O}_{2}{ }^{\bullet}$ and $\mathrm{HO}_{2}{ }^{\bullet}$ radicals, respectively. ${ }^{22,23,33,34}$

Finally, the Au is also likely playing a very subtle role in adjusting the band alignment of the $\mathrm{Au}-\mathrm{ZnO}$ system. The measured bandgap for $\mathrm{Au}-\mathrm{ZnO}$ was $383 \mathrm{~nm}(3.23 \mathrm{eV})$ in comparison to $405 \mathrm{~nm}$ (3.06 eV) for $\mathrm{ZnO}$ (Figure 6.13). This bandgap increase could result from a shift of conduction band edge in a more negative direction or shift of valence band 
edge in a more positive direction. ${ }^{4}$ It is also plausible that both the conduction and valence bands could shift simultaneously in these respective directions to account for the increased bandgap noted. Any shift of the conduction band into more negative direction or any shift of the valence band into more positive direction will improve the energetic band alignment needed for the generation of the $\mathrm{O}_{2}{ }^{\circ}, \mathrm{HO}_{2}{ }^{\circ}$, and $\mathrm{OH}^{*}$ radicals discussed above (see also Figure 6.34). While it can be somewhat counterintuitive, this is an example where a bandgap increase, regardless of whether it occurs through conduction or valence band manipulation, can improve photoactivity.

\subsection{Conclusions}

$\mathrm{Au}-\mathrm{ZnO}$ heterostructures were synthesized using a literature recipe that has been modified to reduce the amount of organic surfactants. The resulting $\mathrm{Au}-\mathrm{ZnO}$ heterostructures have a nanopyramid geometry composed of $\mathrm{ZnO}$ with $\mathrm{Au}$ nanoparticles attached to the basal surface. The Au-ZnO nanopyramids didn't show plasmonic heating effect as expected. There are several possible reasons for that but still the exact reason is unclear. According to the TEM images, Au nanoparticles are not embedded in the $\mathrm{ZnO}$ basal surface and they are just sitting at the surface. Since this reduces the surface contact plasmonic heat transfer to the $\mathrm{ZnO}$ nanoparticle via conduction might be not efficient. A large portion of generated heat at the surface of the Au nanoparticle may be lost in to the air. Also even heat efficiently transfer from $\mathrm{Au}$ to $\mathrm{ZnO}$, there might be a thermodynamic barrier for the catalytic activity of the $\mathrm{ZnO}$ surface which is not possible to overcome in this situation. Finally, the remaining organic molecules even after plasma etching that might affect to the heat transfer 
or catalytic activity. Also, $E_{2}($ high $)$ mode of $\mathrm{ZnO}$ was clearly observed for pure $\mathrm{ZnO}$ but it wasn't visible for Au-ZnO in Raman spectra. But reason for this is unclear.

The (002) planes of $\mathrm{ZnO}$ expand by $\sim 3.5 \%$ in the first $\sim 1.5 \mathrm{~nm}$ of the interface and approach bulk values of $2.60 \AA$ A towards the apex of the nanopyramid. The band gap of $\mathrm{ZnO}$ increases from $405 \mathrm{~nm}(3.06 \mathrm{eV})$ to $388 \mathrm{~nm}(3.23 \mathrm{eV})$ due to heterostructuring with Au. Wavelength dependent photodecomposition studies with methylene blue indicate that direct gap excitation of the $\mathrm{ZnO}$ component of the heterostructure is required to decompose the dye. $\mathrm{Au}-\mathrm{ZnO}$ heterostructures decompose the dye 6 times faster than simple $\mathrm{ZnO}$. The improved photocatalytic activity of $\mathrm{Au}-\mathrm{ZnO}$ is largely attributed to the physical separation of photoexcited electrons and holes caused as electrons are injected from the conduction band minimum of $\mathrm{ZnO}$ to the Fermi level of Au which leads to facile generation of radicals in solution which decompose the dye. It was also hypothesized that the slight increase in $\mathrm{ZnO}$ bandgap in the heterostructure also contributes to the faster degradation kinetics by improving conduction and valence band energetics for injecting electrons and/or holes, respectively, into solution/reactants. Likewise, it was also hypothesized that heterostructuring $\mathrm{ZnO}$ with $\mathrm{Au}$ can cause a decrease of the work function of $\mathrm{ZnO}$ which would also improve lead to improved electron injection from the conduction band to electron acceptors. 


\section{References}

1. K. Flomin, I. Jen-La Plante, B. Moshofsky, M. Diab and T. Mokari, Nanoscale, 2014, 6, 1335-1339.

2. P. Li, Z. Wei, T. Wu, Q. Peng and Y. Li, Journal of the American Chemical Society, 2011, 133, 5660-5663.

3. K. X. Yao, X. Liu, L. Zhao, H. C. Zeng and Y. Han, Nanoscale, 2011, 3, 4195-4200.

4. W. He, H.-K. Kim, W. G. Wamer, D. Melka, J. H. Callahan and J.-J. Yin, Journal of the American Chemical Society, 2013, 136, 750-757.

5. S. Peng, Y. Lee, C. Wang, H. Yin, S. Dai and S. Sun, Nano Res., 2008, 1, 229-234.

6. P. Li, D. Wang, Z. Wei, Q. Peng and Y. Li, Chemistry - A European Journal, 2013, 19, $3735-3740$.

7. W. Wu, L. Liao, S. Zhang, J. Zhou, X. Xiao, F. Ren, L. Sun, Z. Dai and C. Jiang, Nanoscale, 2013, 5, 5628-5636.

8. H. Yin, K. Yu, C. Song, R. Huang and Z. Zhu, ACS Applied Materials \& Interfaces, 2014, 6, 14851-14860.

9. P. R. Ohodnicki, M. P. Buric, T. D. Brown, C. Matranga, C. Wang, J. Baltrus and M. Andio, Nanoscale, 2013, 5, 9030-9039.

10. M. Khan, C. Wei, M. Chen, J. Tao, N. Huang, Z. Qi and L. Li, Journal of Alloys and Compounds, 2014, 612, 306-314.

11. Z. L. Wang, J. Phys. Chem. B, 2000, 104, 1153-1175.

12. M. Shim and H. McDaniel, Current Opinion in Solid State and Materials Science, 2010, 14, 83-94. 
13. M. R. Daymond, M. A. M. Bourke, R. B. VonDreele, B. Clausen and T. Lorentzen, J. Appl. Phys., 1997, 82, 1554-1562.

14. N. C. Popa and D. Balzar, J. Appl. Crystallogr., 2001, 34, 187-195.

15. A. Leineweber, Z. Kristall., 2011, 226, 905-923.

16. Z. Khusaimi, M. H. Mamat, N. Abdullah and M. Rusop, Journal of Nanomaterials, 2012, 2012, 8 .

17. Z. Han, L. Wei, Z. Zhang, X. Zhang, H. Pan and J. Chen, Plasmonics, 2013, 8, 11931202.

18. M. Misra, P. Kapur and M. L. Singla, Applied Catalysis B: Environmental, 2014, 150151, 605-611.

19. J. Lee, H. S. Shim, M. Lee, J. K. Song and D. Lee, J. Phys. Chem. Lett., 2011, 2, 28402845.

20. S. A. Ansari, M. M. Khan, S. Kalathil, A. Nisar, J. Lee and M. H. Cho, Nanoscale, 2013, 5, 9238-9246.

21. N. Udawatte, M. Lee, J. Kim and D. Lee, ACS Applied Materials \& Interfaces, 2011, 3, 4531-4538.

22. Q. Wang, B. Geng and S. Wang, Environmental Science \& Technology, 2009, 43, 89688973.

23. C. Mondal, J. Pal, M. Ganguly, A. K. Sinha, J. Jana and T. Pal, New Journal of Chemistry, 2014, 38, 2999-3005.

24. P. Fageria, S. Gangopadhyay and S. Pande, RSC Advances, 2014, 4, 24962-24972.

25. C. Wang, O. Ranasingha, S. Natesakhawat, P. R. Ohodnicki, M. Andio, J. P. Lewis and C. Matranga, Nanoscale, 2013, 5, 6968-6974. 
26. S. K. Cushing and N. Wu, in Interface, The Electrochemical Society, 2013, pp. 63-67.

27. W. Hou, W. H. Hung, P. Pavaskar, A. Goeppert, M. Aykol and S. B. Cronin, ACS Catalysis, 2011, 1, 929-936.

28. A. J. Nozik and R. Memming, J. Phys. Chem., 1996, 100, 13061-13078.

29. A. Houas, H. Lachheb, M. Ksibi, E. Elaloui, C. Guillard and J.-M. Herrmann, Applied Catalysis B: Environmental, 2001, 31, 145-157.

30. E. C. H. Sykes, F. J. Williams, M. S. Tikhov and R. M. Lambert, J. Phys. Chem. B, 2002, 106, 5390-5394.

31. Z. Zhang, G. Meng, Q. Xu, Y. Hu, Q. Wu and Z. Hu, The Journal of Physical Chemistry C, 2009, 114, 189-193.

32. X. Wang, C. J. Summers and Z. L. Wang, Applied Physics Letters, 2005, 86, 013111.

33. M. Ahmad, S. Yingying, A. Nisar, H. Sun, W. Shen, M. Wei and J. Zhu, Journal of Materials Chemistry, 2011, 21, 7723-7729.

34. X. Hou, Materials Letters, 2015, 139, 201-204. 
Synthesis, characterization and photocatalytic activity of $\mathrm{Au}-\mathrm{ZnO}$ heterostructures

Oshadha Kanchana Ranasingha

Dissertation submitted to the Eberly College of Arts and Sciences at West Virginia University in partial fulfillment of the requirements for the degree of

Doctor of Philosophy in Physics

Department of Physics and Astronomy

APPROVAL OF THE EXAMINING COMMITTEE
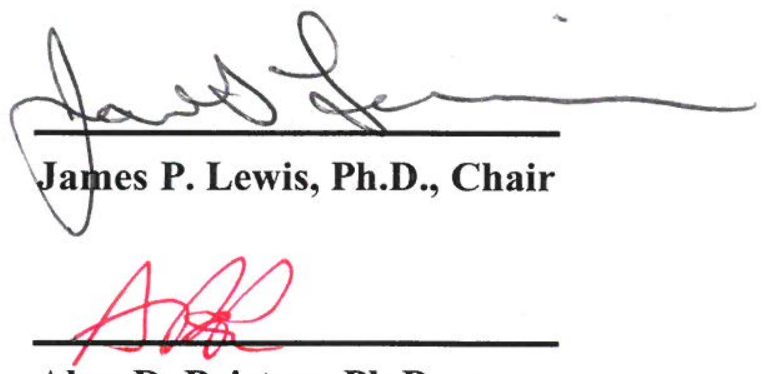

Alan D. Bristow, Ph.D.

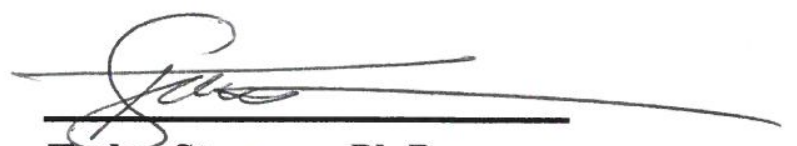

Tudor Stanescu, Ph.D.

$04 / 29 / 2015$

Date

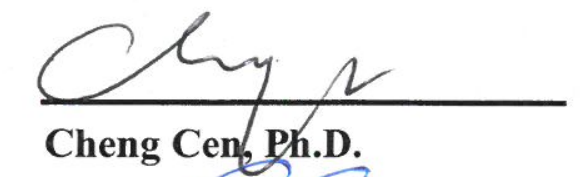

Cheng Cen, Ph.D.

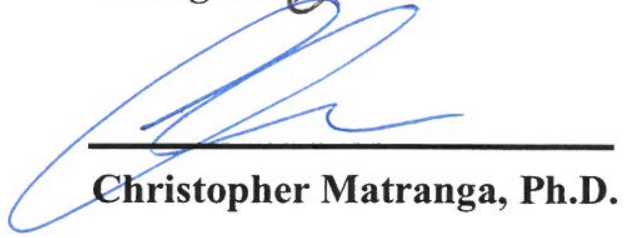

